\title{
VIETNAMESE EFL TERTIARY TEACHERS' ENGLISH COMMUNICATION IN NON-ENGLISH MAJOR CLASSES: \\ A CASE STUDY
}

\author{
BY
}

TRAN THI THUY LIEN

\begin{abstract}
A thesis
submitted to Victoria University of Wellington

in fulfilment of the requirements for the degree of

Doctor of Philosophy
\end{abstract}

Victoria University of Wellington

2020 


\begin{abstract}
The way English is taught at all educational levels has been a matter of big concern in Vietnam. This is clearly shown by the National Foreign Languages Project 2020 (phases 2008-2016 and 2017-2025) which aims to renovate all aspects of English teaching including teaching facilities, teacher proficiency, curriculum, assessment methods, and learning outcomes, particularly in tertiary English teaching (Vietnamese Government, 2008). Teachers' classroom English communication is an important part of English teaching and learning; thus, closely examining how they use classroom English and communication strategies is a necessity. However, most international and Vietnamese research of English communication in English as a Foreign Language (EFL) teaching contexts has focused on the perspectives of learners, with limited attention given to the role of teachers. This thesis fills this gap by examining tertiary teachers' practices of English communication in relation to learners' perspectives.
\end{abstract}

This study investigated how English communication was used by five Vietnamese lecturers of English who were teaching non-English major students at two public colleges in Vietnam. This research adopted a mixed methods and qualitative dominant approach. The data were collected via classroom observations, survey questionnaires, individual interviews with lecturers, and focus group interviews with students. Findings reveal that, while most of the lecturers said they used more English than Vietnamese, classroom observation and student interview data suggested that they spent marginally less time speaking English than Vietnamese. Both lecturers and students shared viewpoints on the benefits of an English-only approach, but many did not think this approach would be applicable and effective in classes. Both lecturers and students believed that lecturers' choice and use of classroom language was predominantly influenced by the desire to ensure comprehension and to provide concern to students. Findings further show seven key communication strategies used by the lecturers, with humour having not been previously identified in communication strategy research.

The lecturers' roles as language users and language analysts are assumed to be mutually interconnected to lead to their practices of communication strategies; and the role of language teachers with their pedagogical learner knowledge ${ }^{1}$ shaped their perceptions on the functions and usages of communication strategies. Mismatches between the lecturers' and students'

\footnotetext{
${ }^{1}$ Pedagogical learner knowledge refers to teachers' knowledge of the learners in all their richness and complexity as well as teachers' ability to accommodate their subject-matter knowledge and knowledge of L2 teaching to their learners' varied learning trajectories (Le, 2020).
} 
perceptions of classroom English communication were also identified. Those mismatches were caused by a limitation on communication at the interpersonal level between the lecturers and students and the particularly hierarchical and formal teacher-learner relationship in Vietnamese culture. To minimise those perceptual gaps, it is recommended that lecturers need to consider the perspectives of students to know what they expect to learn and how to learn that effectively. Lecturers' classroom communication styles and strategies are also shown to be important to help alleviating those perceptual mismatches. It is also suggested that EFL classrooms should offer features of a supportive and motivating environment such as a well-designed classroom layout, teachers' systematic corrective feedback, less asymmetrical power, and plentiful interaction opportunities. In the communicative and learner-centred teaching approaches, EFL teaching needs to be innovative to better engage and motivate students and to create more learning opportunities.

Taken as a whole, this thesis suggests that socially affective classroom culture plays an important role in students' foreign language (FL) and second language (L2) learning and development. A positive lecturer-student relationship, a supportive learning environment, and interaction opportunities are the three main factors that can mediate and construct students' FL/L2 learning. This study also emphasises the essential role of lecturers in shortening the perceptual gaps between them and students and opening learning space for students. Lecturers' classroom communications strategies are used for communicative, affective, motivational, and pedagogical purposes and can be converted into students' learning strategies with mediation tools. To improve EFL teaching and learning, this study also recommends an English-dominant teaching policy, job-oriented and communicative-based syllabus and assessment, and frequent teacher self-reflection and students' feedback. Lastly, the research has useful implications for EFL teacher education and proficiency development. 


\section{Acknowledgement}

This $\mathrm{PhD}$ dissertation is, for me, a journey of interesting discovery and fruitful collaboration. I would not have been able to complete this work without the guidance, support, and participation of those people whom I thank below.

Firstly, I would like to express my profound gratitude to my supervisors, Dr. Jae Major, Assoc. Prof. Jenny Ritchie, and Assoc. Prof. Robin Averill, who have been supporting me throughout this research project. Their critical minds, dedicated feedback, and overall knowledge of the research field have developed my academic skills. Their patience, sympathy, encouragement, and cultural understanding made this journey a memorable experience for me, an international married student whose language and culture is not English.

Secondly, I am especially indebted to the Vietnamese government and Victoria University of Wellington for granting me a doctoral scholarship to pursue this study. My appreciation is also extended to the staff at the school of Education and the faculty of Graduate Research for their support during my studying and living in New Zealand.

My sincere thanks also go to Dr. Deborah Laurs and Louise Falepau for their useful advice on my writing; Dr. Lisa Woods for help with statistical analysis; Dr. Thanh Nguyen for helping with data analysis; Dr. Le Nguyen for checking translated data; Agnes Terraschke for proofreading my thesis; Hoang Thi Thu Ha for allowing me to observe her English evening classes and conduct my first classroom observation pilot study; and Ngo Nga, Vo Tho, and Tran Ngoc Minh for their friendship and useful assistance.

I would like to express my heartfelt gratitude to the Vietnamese lecturers and students who participated in my research. Without their kind sharing and enthusiastic cooperation, the completion of this thesis would have been unrealistic. I sincerely thank the heads of the two colleges and the deans of their English faculties in Danang, Vietnam, for their permission and encouragement during my data collection.

Last but not least, I am thankful my parents, Mr. Tran Lac and Mrs. Nguyen Thi Loi, my only sister Ms. Tran Thi Thuy Loan, and parents-in-law, Mr. Ho Lau and Mrs. Dinh Thi Thu who gave so much love and endorsement throughout my study. I particularly acknowledge my husband, Mr. Ho Thanh Dung, and my beloved princess, Ho Tran Thuy Duong, who have been always by my side and motivated me throughout these years. I dedicate this work to them all for their inexhaustible love and care during these oversea tough times and always. 


\section{Table of Contents}

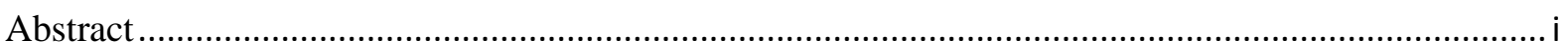

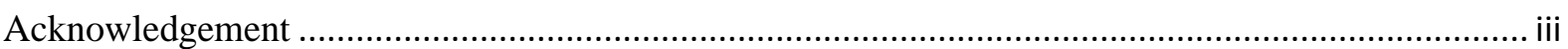

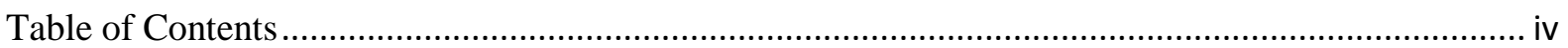

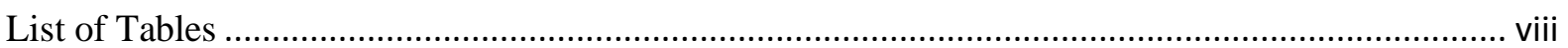

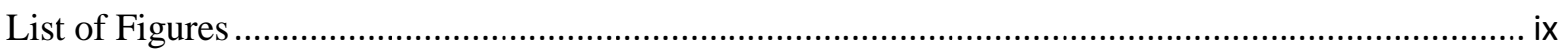

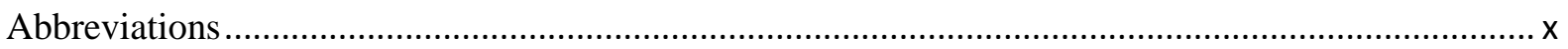

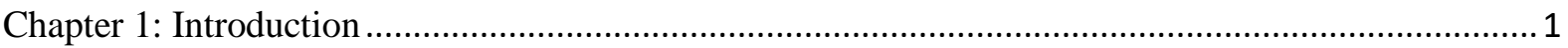

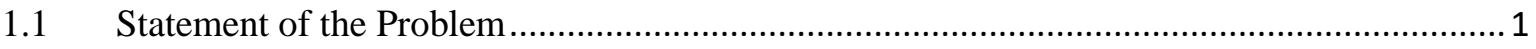

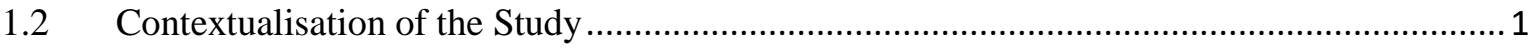

1.3 Researcher's Position and Motivation ................................................................. 2

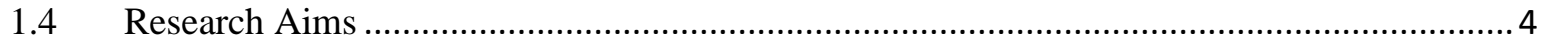

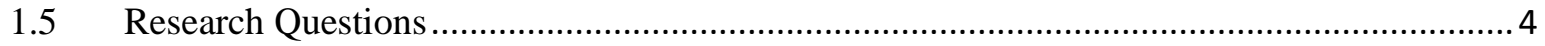

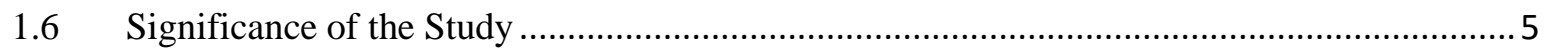

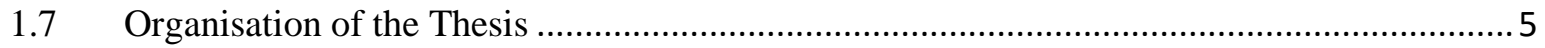

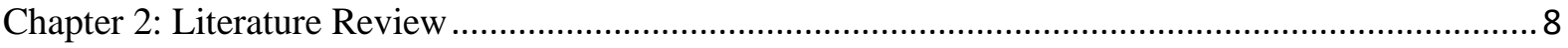

2.1 Conceptual and Theoretical Frameworks in Second Language Acquisition ...........................8

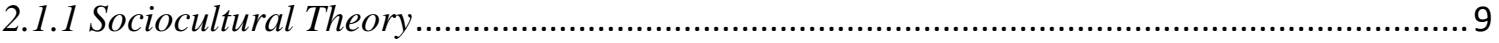

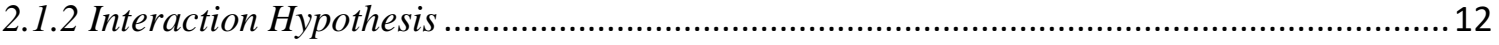

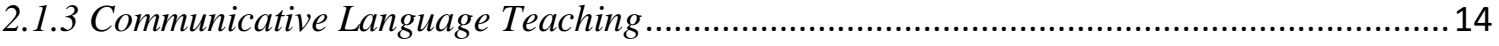

2.1.4 How do the Sociocultural Theory, Interaction Hypothesis, and Communicative Language

Teaching Approach Frame this Study?..................................................................... 17

2.2 Communication in L2/FL Teaching and Learning .................................................. 18

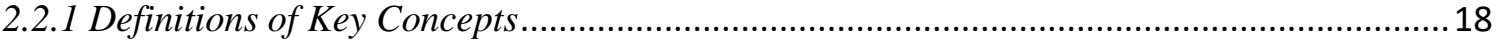

2.2.2 Communication Strategy Taxonomies................................................................ 20

2.2.3 Empirical Research in Teachers' English Communication and Strategies in EFL Teaching

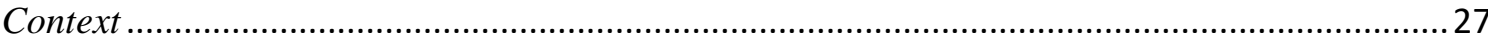

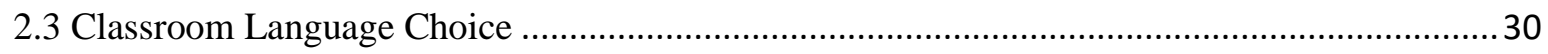

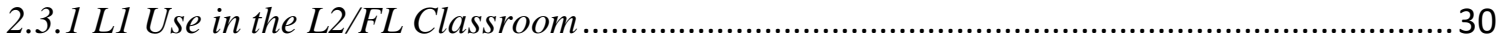

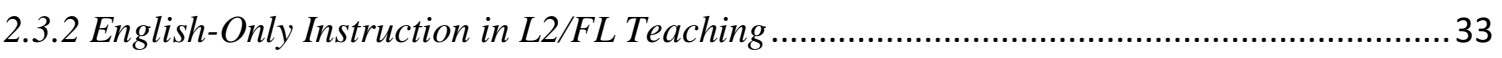

2.4 Teacher Language Awareness and Perceptions ............................................................. 36

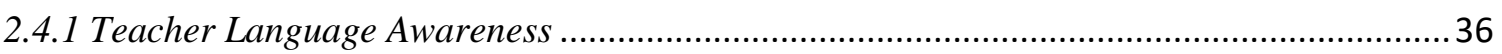

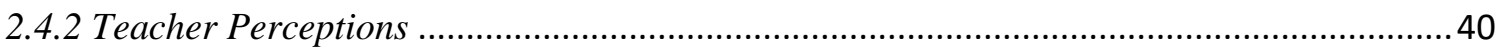

2.4.3 How Language Awareness and Perceptions Influence Teachers' Communication Strategies

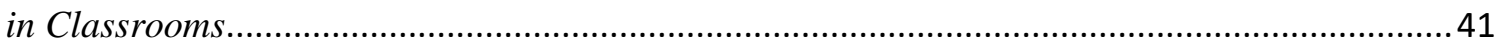


2.5 Affective Factors in Second Language Teaching and Learning

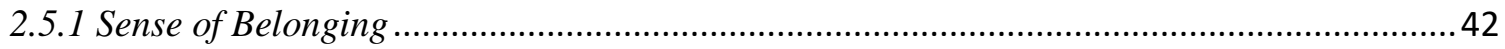

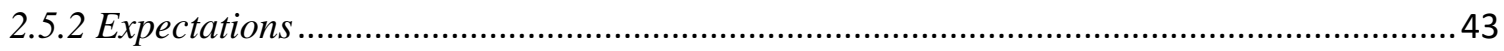

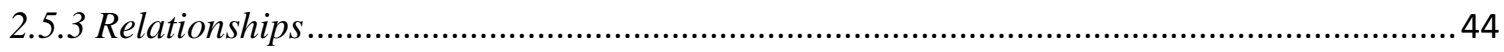

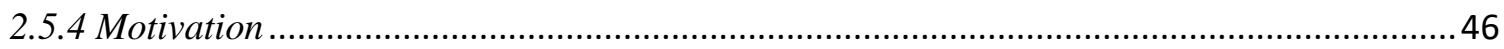

Chapter 3: Methodology and Methods of the Research...................................................................... 49

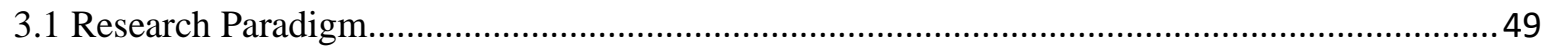

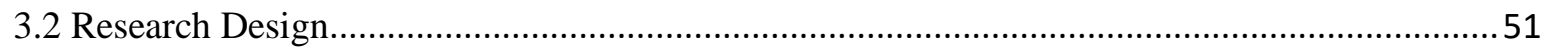

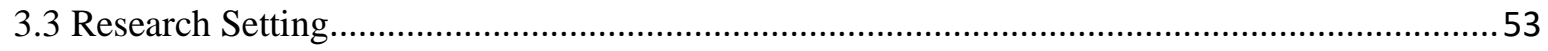

3.3.1 The National Foreign Languages Project 2020 ............................................................... 53

3.3.2 The Two Research Sites ................................................................................................... 54

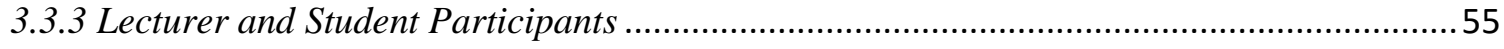

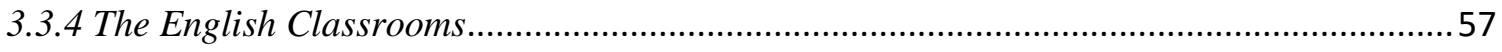

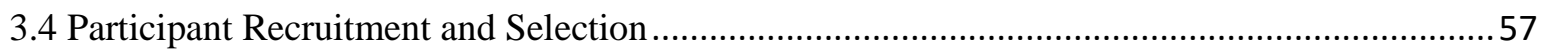

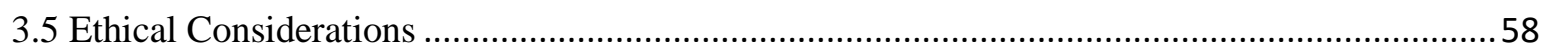

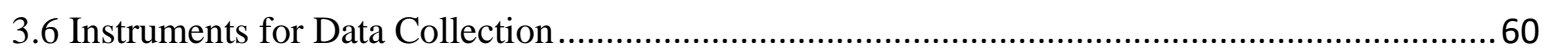

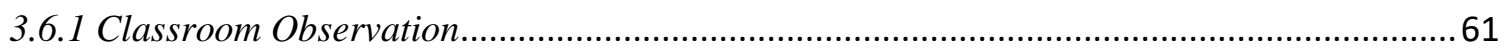

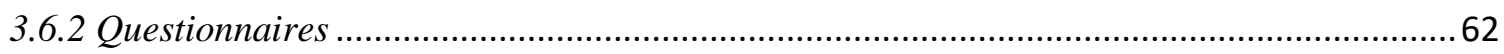

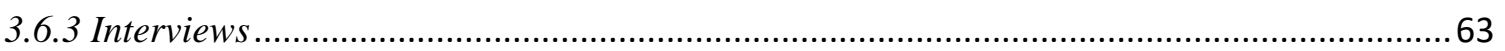

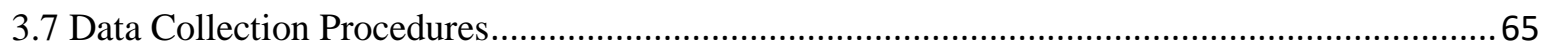

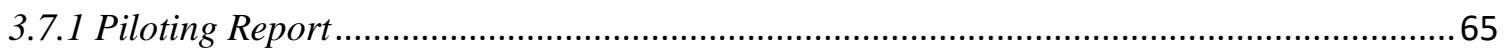

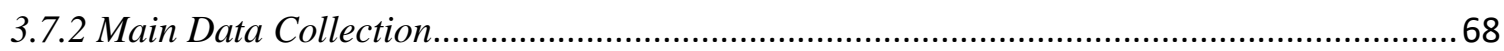

3.8 The Use of Vietnamese and English during Data Collection, Analysis, and Report .................. 71

3.8.1 Vietnamese for Data Collection and Coding ......................................................................... 72

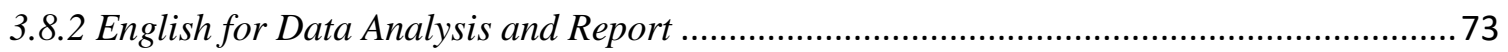

3.8.3 Translation of Quotations and Related Issues ................................................................. 73

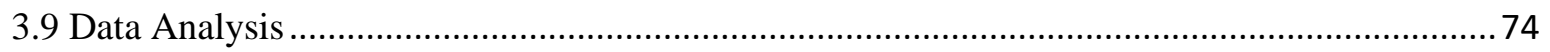

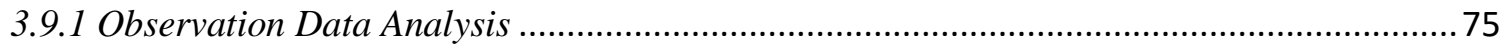

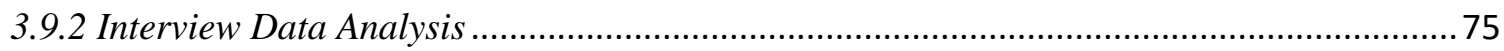

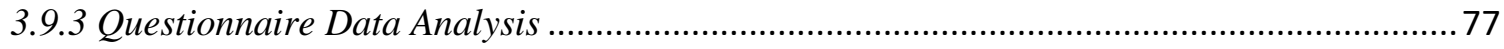

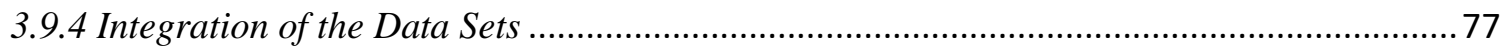

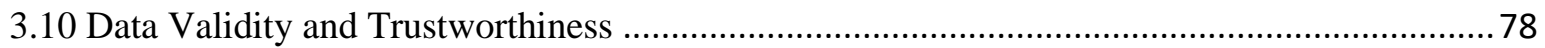

3.10.1 Validity of Quantitative and Qualitative Data .............................................................. 78

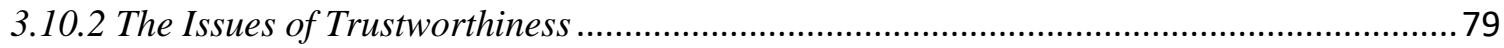

3.10.3 Potential Limitations in the Research Methodology ....................................................... 80

Chapter 4. An Overall Picture of Lecturers' English Communication ................................................ 82 
4.1 Proportion of English and Vietnamese Spoken by Lecturers in Non-English Major Classes .... 82

4.2 Lecturers' and Students' Perceptions of an English-only Teaching Approach........................... 84

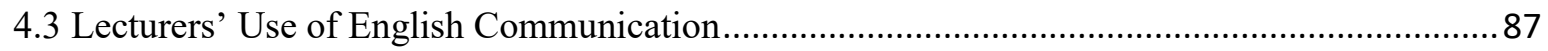

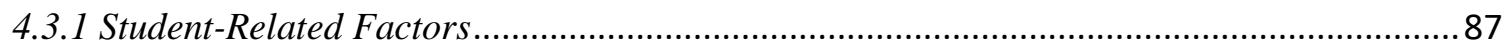

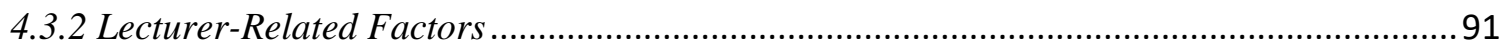

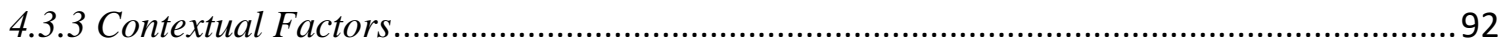

4.4 Students' Comprehension of Lecturers' English and their Learning Motivation ....................... 94

4.4.1 Students' Comprehension Levels, Perceptions on Learning Improvement, and Motivation to

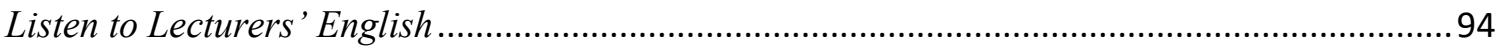

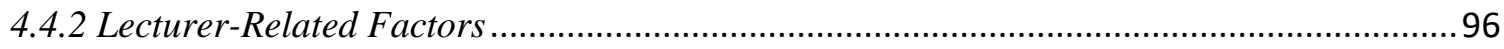

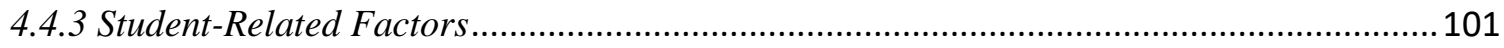

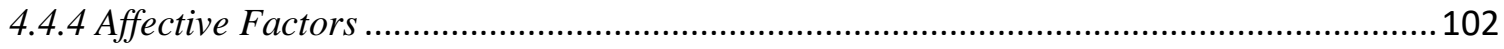

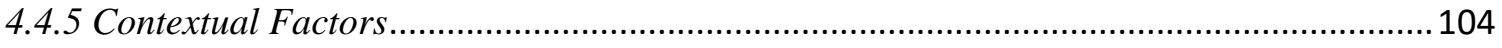

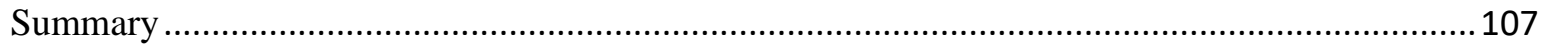

Chapter 5. Lecturers' English Communication Strategies ............................................................ 109

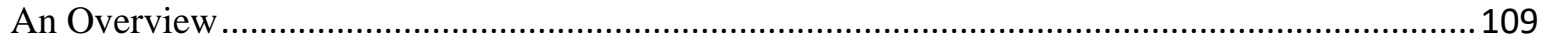

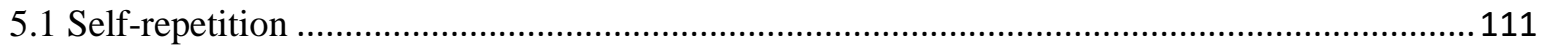

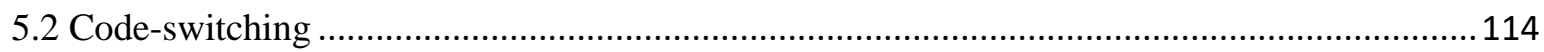

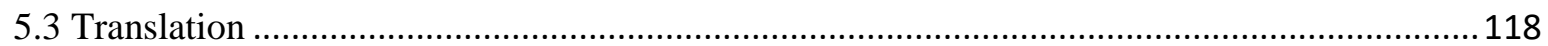

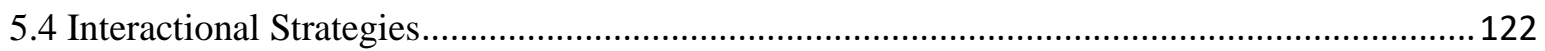

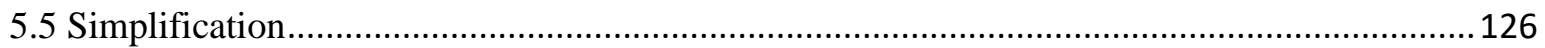

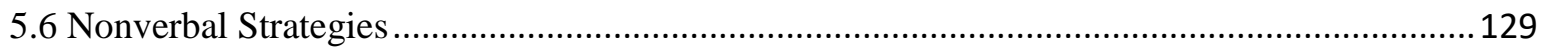

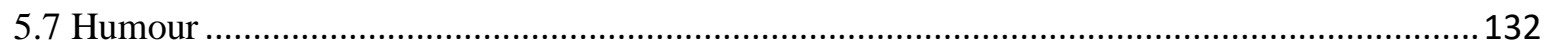

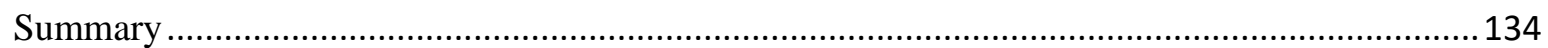

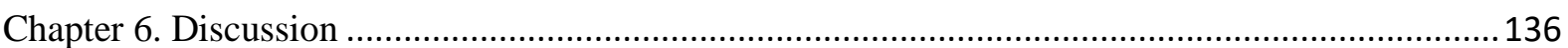

6.1 The Complexity of Language Teacher Roles in the Use and Perceptions of Communication

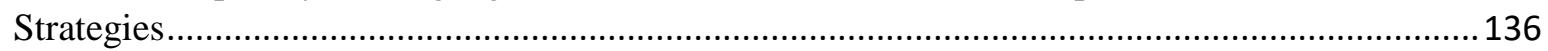

6.1.1 The Two Domains of Teacher Language Awareness in Communication Strategy Practices

6.1.2 Pedagogical Knowledge and Perceptions of Communication Strategies ..........................138

6.1.3 An Emerging Taxonomy from an Integrated Perspective ...............................................141

6.2 Mediation Tools in EFL Classroom Teaching and Learning ................................................... 145

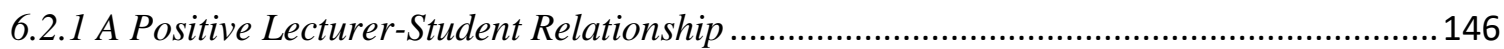

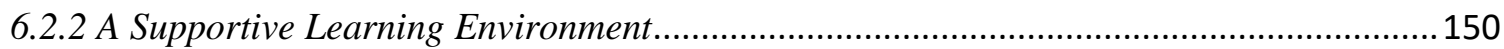

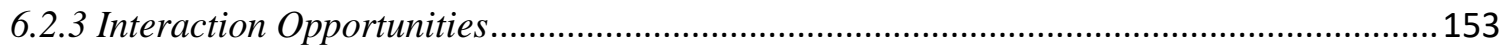

6.3 Tensions between How Lecturers See Classroom Communication and How Students Perceive What Works for Them 155 
6.3.1 The Amount and Use of L1 156

6.3.2 The Use of Communication Strategies... 157

6.3.3 The Outcomes of Teaching and Learning 159

6.3.4 What Might Cause the Perceptual Gaps between Lecturers and Students? 161

Summary 163

Chapter 7: Implications, Recommendations, and Limitations 165

7.1 Addressing the Research Questions 165

7.2 EFL Teachers' Communication Strategies in Non-English Major Classes ............................. 166

7.3 EFL Classroom Dynamics for Non-English Major Students.................................................... 167

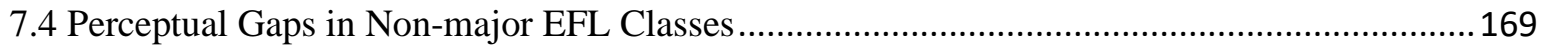

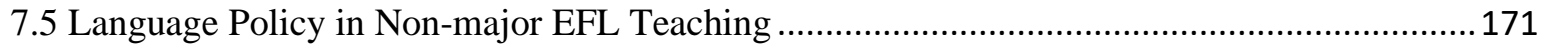

7.6 EFL Syllabus and Assessment for Non-English Major Students............................................173

7.7 EFL Teacher Self-reflection and Students’ Feedback ..........................................................175

7.8 EFL Teacher Education and Competence Standardisation................................................... 177

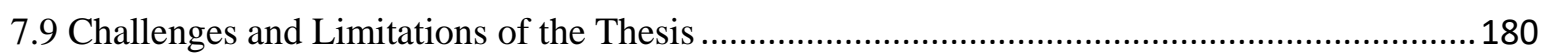

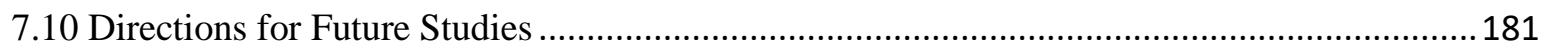

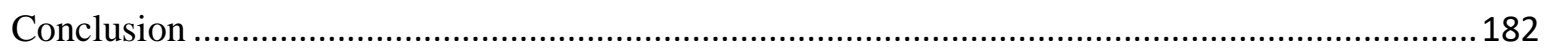

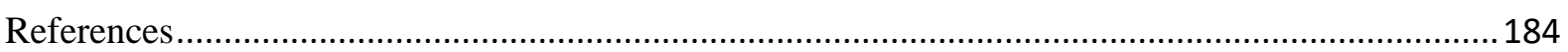

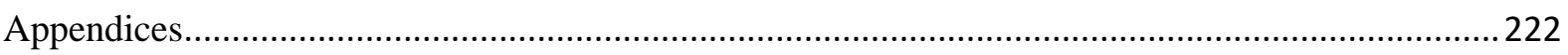




\section{List of Tables}

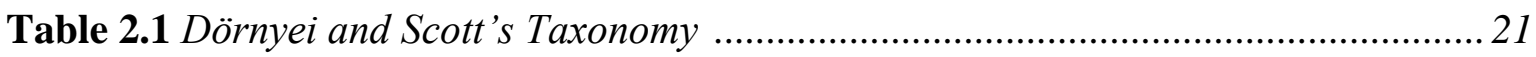

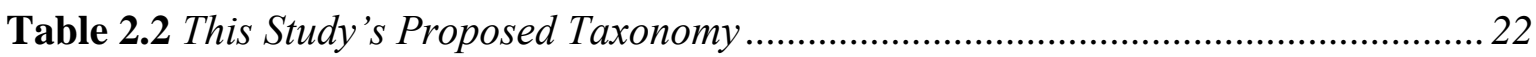

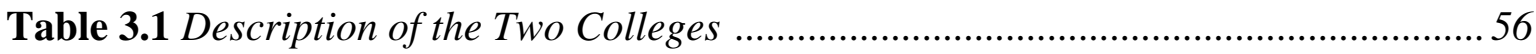

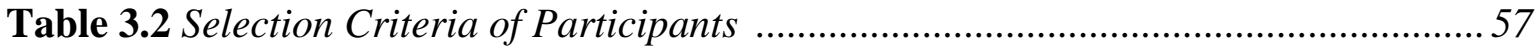

Table 3.3 The Research Questions in Relation to the Data Collection and Analysis ........ 60

Table 3.4 Classroom Observation Contextual Information ..............................................69

Table 4.1 Student Perceptions by Motivation Levels When Listening to Lecturers Speaking

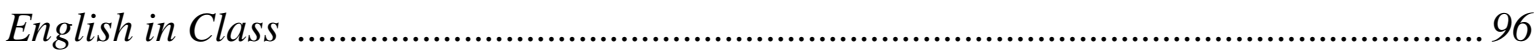

Table 4.2 Student-Related Barriers to Students' Comprehension of Lecturers' English Speaking 102

Table 5.1 Frequency and Percentage of Strategies Used by Lecturers from Classroom Observation Data 109

Table 5.2 Frequencies of Strategies Used by Lecturers from Classroom Observation Data 110

Table 7 Answers to Research Questions 165 


\section{List of Figures}

Figure 3.1 The Convergent Parallel Mixed Methods Research Design ...........................51

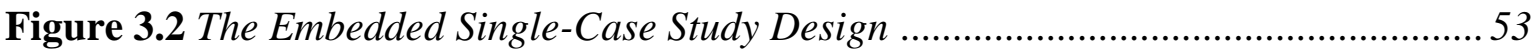

Figure 4.1 Percentages of Lecturers' Use of English (L2) versus Vietnamese (L1)...........82

Figure 4.2 How Much do Students Understand Lecturers' English Speaking and How Much does It Help Them Improve Communication Skills? 95

Figure 4.3 What Motivates Students to Listen to Lecturers' English? 97

Figure 4.4 What Demotivates Students to Listen to Lecturers' English? 97

Figure 6 Disciplines and Functions of EFL Lecturers' English Communication Strategies 145 


\section{Abbreviations}

\begin{tabular}{|c|c|c|}
\hline Abbreviation & Full term & Definition \\
\hline CLT & $\begin{array}{l}\text { Communicative Language } \\
\text { Teaching }\end{array}$ & $\begin{array}{l}\text { An approach to language teaching that offers learners the } \\
\text { opportunity to practice their skills and assume different } \\
\text { interactional roles through the use of communicative } \\
\text { activities and tasks (Albahri et al., 2018). }\end{array}$ \\
\hline CSs & Communication Strategies & $\begin{array}{l}\text { Techniques speakers use when facing communicative } \\
\text { problems or trying to achieve communicative goals } \\
\text { (Yang \& Gai, 2010). }\end{array}$ \\
\hline EFL & $\begin{array}{l}\text { English as a Foreign } \\
\text { Language }\end{array}$ & $\begin{array}{l}\text { A term used to describe the teaching of English to those } \\
\text { whose English is not the official or main language } \\
\text { (Cambridge Dictionary, 2017). }\end{array}$ \\
\hline FL & Foreign Language & $\begin{array}{l}\text { "A language which is not the native language of large } \\
\text { numbers of people in a particular country or region, is not } \\
\text { used as a medium of instruction in schools and is not } \\
\text { widely used as a medium of communication in } \\
\text { government, media" (Richards \& Schmidt, 2010, p. 224). }\end{array}$ \\
\hline L1 & First Language & $\begin{array}{l}\text { A language which is learnt during learners' early } \\
\text { childhood and passes from generation to generation } \\
\text { (Sinha et al., 2009). }\end{array}$ \\
\hline L2 & Second Language & $\begin{array}{l}\text { A language other than the mother tongue that might be } \\
\text { the official language or one of the official languages in } \\
\text { the country where it is learned; it can be used in that } \\
\text { country for public communication or trade and education } \\
\text { purposes (Collins English Dictionary, 2019). }\end{array}$ \\
\hline SCT & Sociocultural Theory & $\begin{array}{l}\text { One of the frameworks of this study, which positions } \\
\text { learning within social interaction and views human } \\
\text { cognition as mediated by language, materials (e.g. signs), } \\
\text { and other people (Foster \& Ohta, 2005; Vygotsky, 1978). }\end{array}$ \\
\hline SLA & $\begin{array}{l}\text { Second Language } \\
\text { Acquisition }\end{array}$ & $\begin{array}{l}\text { A theoretical framework that emphasises how people } \\
\text { learn and adopt an additional language other than their } \\
\text { first language (Gass, 2008; Krashen, 1985; Long, 1983). }\end{array}$ \\
\hline
\end{tabular}




\subsection{Statement of the Problem}

\section{Chapter 1: Introduction}

As an English as a foreign language (EFL) lecturer in Vietnam, I have been aware that using English as a language of communication in the classroom has a significant influence on the learning process in my classes. English as a medium of instruction courses along with an increased investment in educational programs and facilities have been offered in both public and private tertiary institutions in Vietnam since 2008 (Vietnamese Government, 2008). Up to the end of 2019, more than 20 universities in Vietnam had introduced English as a medium of instruction in many different training fields and disciplines. The implementation of this instruction was criticised for increasing demands on teachers without providing the necessary support structures that would allow teachers to gain adequate English language proficiency levels and learn relevant teaching skills and techniques (H. T. Nguyen et al., 2016). Also, Vietnamese lecturers and students face many other challenges related to the teaching and learning culture, including large classes, lack of teaching facilities, and students' low learning motivation. These have hindered the success of English medium instruction and the quality of overall English language education at colleges and universities.

Tertiary education directly provides human resources for the society; and in order to do this, it is required to equip students with different necessary skills and relevant knowledge bases. In this age of globalisation, English is an important key to many attractive career opportunities and is indispensable for those wanting to work in international companies and/or to become global citizens. Thus, English is not only an essential tool for English majors but also for nonEnglish major students. Compared with majors, non-English major students can have lower levels of intrinsic motivation and lack adequate preparedness for their learning (Ngo et al., 2017), which may lead to their lack of interest during lessons. Hence, the enhancement of English teaching efficiency in non-English major classes seems to be harder. With the above challenges, this study explores how Vietnamese lecturers' English communication in the classroom can enhance non-English major students' learning.

\subsection{Contextualisation of the Study}

The challenges of globalisation have pushed Asian countries, in general, and Vietnam, in particular, to improve the quality of education to meet international standards, which would enhance its competitive competence with that of developed nations (Le \& Chen, 2019). One of the key concerns regarding the quality of Vietnamese education has been the reformation of 
English teaching and learning. English as a foreign language is taught widely as a compulsory subject in many educational institutions in Vietnam, from primary schools to tertiary institutions. The Vietnamese government has set out a number of policies and initiatives in order to improve the quality of EFL teaching and learning in the Vietnamese education system. For instance, the National Foreign Languages Project 2020 was signed by the Prime Minister in 2008 with one of its important objectives being that learners at all school levels are expected to use English fluently by the year 2020 (Vietnamese Government, 2008). However, it was announced on public media in late 2016 that this National Foreign Languages Project is officially on the edge of failure (Yen Nguyet, 2016). Seeing that there were still another four years to go, the Ministry of Education and Training petitioned the Government for the extension of the project in 2017. The Government decided to extend the National Foreign Languages Project to the second phase from 2017 to 2025 (Vietnamese Government, 2017).

In 2020, the last year of the main project phase 2008-2020, it was widely reported in public media and education conferences that many goals set forth in this project were not achieved, which was due to the unrealistic goals and a lack of appropriate solutions and proper orientation for its implementation. A number of other challenges for English teaching in Vietnam were also identified, such as inadequacies in the training of English teachers (Nguyen \& Bui, 2016) or the limited opportunities to use authentic sources from foreign communities such as nativeEnglish speaking teachers (Trinh \& Mai, 2019). All of these have hampered English teaching and learning at all levels of education, especially at tertiary institutions, which indicates the need for further research and discussion among educators, administrators, and researchers to find solutions to enhance the effectiveness and quality of English language teaching and learning.

\subsection{Researcher's Position and Motivation}

I began to learn English in 1998 as a compulsory subject in secondary school. Until I entered my first year as an English-major student at Da Nang College of Foreign Languages, I had been taught English via Vietnamese. I remember that, in my first year at university, a lot of my classmates were really shocked to hear our lecturers speak English and worried about being expected to respond in English during the first lessons. However, with time, we overcame our initial anxiety, felt more confident, and started to speak, listen, write, and even think in English after the first two years of being mainly taught in English. It is this university experience that has led me to the belief that teachers' use of English in the classroom positively affects students' learning motivation and communication skills, regardless of students' ages and 
levels. Also, my university English learning made me realise that with interest and a positive attitude, students can quickly adapt to an English-only or English-dominant teaching environment as well as other innovations.

I started my English teaching career at Korea-Vietnam Friendship Information Technology College, a public college in Danang city, in 2009. All the students at my college were nonEnglish majors and their admission levels of English were very low because they did not have to take English entrance tests to be admitted to the college. The majority of them had elementary level English, which was equivalent to level 1 and 2, according to the Common European Framework of Reference for Languages. Hence, using English to teach them English was a big challenge for me. I remember experiencing students' different reactions, such as asking me to switch to Vietnamese or being shy with red faces when I spoke English to them. I then tried to talk to them to explain the benefits of using English to teach English and encouraged them to have more positive attitude towards my English speaking. During classes, I tried to combine English and Vietnamese and applied different strategies to help students better understand my English, which reduced my students' negative reactions. As a result, they gradually developed their listening skills and spoke a little English with correct pronunciation and more confidence. Some of the students even sometimes chatted with me in English during breaks. These practical experiences of teaching non-English majors, combined with the previous teacher professional training years, helped me realise that the two most valuable keys to successful EFL teaching are motivational and communication strategies.

I had the opportunity to study for a Master's degree in English in 2010 and undertook a TESOL training course by an Australian organisation in 2016. Next, I was lucky to be offered a doctoral scholarship by the Vietnamese government and Victoria University of Wellington to study in New Zealand, an English-speaking country. This was a great milestone in my academic as well as professional journey as it allowed me to conduct research in the field of second language acquisition and EFL teaching, as well as to enhance my English competence, which is of great importance for my pending practical teaching and future scholarly work.

All the above-mentioned factors have motivated me to conduct this current study. My experiences as an EFL learner, a lecturer, and a researcher have given me a suitable background and strong motivation to complete this thesis. For me, this thesis is not merely a compulsory requirement for the $\mathrm{PhD}$ degree, but it is my real passion motivated by my love for students and my commitment to EFL teaching in my home country. 


\subsection{Research Aims}

This study investigates the English that lecturers use as a medium of teaching and classroombased communication and its impact on their classroom practices at tertiary institutions in Vietnam where English is taught as a non-major subject. To realise that overarching purpose, this thesis firstly explores the balance between Vietnamese (first language) and English (foreign language) used by lecturers. Secondly, it identifies types of lecturers' English communication strategies. Thirdly, the research investigates what lecturers perceive to support and hinder their use of communication strategies and how best to use English in teaching. Lastly, the thesis examines perceptions of students on affordances and barriers to comprehending their lecturers' spoken English and what helps them learn English more effectively.

Beyond these four purposes, this study provides a deeper understanding of many related features that influence EFL teaching and learning, such as the affective domain, the tensions and challenges lecturers face on an interpersonal and professional level, and the expectations of students and lecturers. The thesis' ultimate goal is to propose evidence-based recommendations that could benefit the learning and teaching within Vietnamese EFL classrooms.

\subsection{Research Questions}

Research questions are important for guiding decisions about research design and methods as they help researchers to understand the main procedures involved (Bryman, 2006). Looking back, I believe that the research questions had the greatest influence on my research journey. They implicitly reflected my world view and ultimately determined the selection of the mixed methods paradigm, research design, and data collection instruments (Bryman, 2016). Based on the research goals as described above, the overarching research question is: How do Vietnamese EFL lecturers use English communication in non-English major classes? This key question is further divided into the following four sub-questions:

1. How much Vietnamese versus English do lecturers use?

2. What English communication strategies do lecturers use and how are they used?

3. What are lecturers' perspectives on what affects their English communication in classes? 
4. What are students' perspectives on what helps them better understand their lecturers' English speaking and learn English more effectively?

\subsection{Significance of the Study}

Although there has been much research in classroom communication and communication strategies in language education, previous studies mainly focused on the stance of learners. Therefore, from the perspective of academic contribution, this in-depth study of classroom English communication with a focus on teachers, including a purpose-designed researchinformed communication strategy taxonomy, contributes to the literature in this field. Particularly, the addition of a new element to the existing communication strategy taxonomy, as well as certain distinct functions of EFL lecturers' communication strategies, the findings of this study highlight the need to refocus existing taxonomies so that they are more relevant to L2 or FL classroom contexts.

The findings of this study also lead to useful suggestions for English learning and teaching in the two research contexts to enhance students' speaking and listening competence. This study provides evidence concerning how students perceive their lecturers' English speaking in relation to their learning of English. Since the learner-centred approach has been identified as central in Vietnam (Dang, 2006), recommendations from students offer strong evidence for the lecturers and the tertiary institutions to reinforce suitable and relevant policies and practices to facilitate English teaching and learning. More importantly, because the situations of the two researched contexts are common in language classrooms, the findings of this study can inform policies and practices regarding English education reform in other Vietnamese education contexts. As for those lecturers who do not use much English in non-major classes, the final and completed taxonomy can be a useful guideline for them, which can help them feel more confident and give them ideas for finding opportunities to use more English communication. Also, students' perspectives can be a concrete stimulus for the lecturers to change their practices to better meet the needs of their students and enhance the teaching efficiency. Lastly, when the National Foreign Languages Project 2020 (phases 2008-2016 and 2017-2025) is entering the final years and making a final effort to achieve its goals, recommendations from this research can contribute to the success of this significant project.

\subsection{Organisation of the Thesis}

This thesis is organised into seven chapters, which are briefly described below. 


\section{- Chapter 1: Introduction}

This chapter provides an overall picture of the whole thesis. It explains the research's rationales and establishes that research context as well as the researcher's position and motivation. A description of research purposes, questions, and their significance is also included in this chapter, followed by an outline of the thesis.

\section{- Chapter 2: Literature Review}

This chapter positions the research study within the academic field by presenting and discussing relevant literature from five main areas. The first section explains how the sociocultural theory and interaction hypothesis are relevant to the notion of communicative FL/L2 language teaching and altogether underpin this study. This is followed by a discussion of communication strategies, classroom language choice, teacher language awareness and perceptions, and affective factors in EFL teaching contexts. Throughout the chapter, gaps in the literature are highlighted and linked to the current study.

\section{- Chapter 3: Methodology and Methods of the Research}

This chapter describes the data collection and analysis approaches chosen to address and answer the research questions. Thus, the chapter explains the research paradigm, the mixed methods research design, and relevant data collection methods. It also presents a detailed account of the research settings, selection of participants, ethical issues, and the procedures followed for the pilot study, main data collection, data analysis, data validity and trustworthiness, as well as the challenges and possible limitations related to the methods and circumstances surrounding the data collection.

\section{- Chapter 4: An Overall Picture of Lecturers' English Communication}

This chapter presents the findings with regard to four overall aspects related to the lecturers' use of English communication in EFL classes. Thus, the chapter reports the level of English and Vietnamese spoken by the lecturers in non-English major classes. It then discusses the lecturers' and students' perceptions of the English-only teaching practice, the factors affecting the lecturers' use of English communication in non-English major classes, and the factors having an influence on non-English major students' comprehension of and listening motivation toward their lecturers' English speaking. 


\section{- Chapter 5: Lecturers' English Communication Strategies}

This chapter reports the findings on the lecturers' use of English communication strategies. It focuses on the occurrence, types, and functions of the strategies used. The lecturers' perceptions of their communication strategies use are also covered, in combination with how students perceived the use of strategies that could help them learn English better.

\section{- Chapter 6. Discussion}

This chapter schematically discusses the findings in relation to the mainstream literature on English communication used in FL/L2 classes. It is divided into three main ideas: the complexity of language teacher roles in the use and perceptions of communication strategies; the mediation tools in EFL classroom teaching and learning; and the tensions between how the lecturers see classroom communication and how the students perceive what works for them.

\section{- Chapter 7: Implications, Recommendations, and Limitations}

This final chapter firstly describes the implications and recommendations for EFL teaching and learning within the seven themes. Accordingly, it provides recommendations not only for lecturers but also for the institution and national education policy advisers to enhance the overall efficiency of EFL teaching and learning in Vietnam. The remaining section discusses the challenges that were encountered during the data collection process and the overall limitations of this research, along with directions and suggestion for future studies in the research field. 


\section{Chapter 2: Literature Review}

This chapter is organised into five sections, beginning with an overview of sociocultural theory, interaction hypothesis, and communicative language teaching as the conceptual and theoretical frameworks that inform this study. Key the concepts of classroom communication, communication strategies, and communication taxonomies are then discussed along with reviews of relevant empirical research. Classroom language choice between first language and second language is also reviewed, followed by descriptions of teacher language awareness and perceptions as foundations for data analysis. In the last part of this chapter, concepts related to affective factors in FL/L2 teaching and learning contexts are presented.

\subsection{Conceptual and Theoretical Frameworks in Second Language Acquisition}

Second language acquisition (SLA) has been discussed from different perspectives. Behaviourism posited that L2 learning occurs through imitation of models in the input, repetition of engagement in the target behaviour, and provision of consistent feedback (Vanpatten \& Williams, 2014). However, this theory has been criticised in that imitation is not helpful for learners to deal with authentic situations (Saville-Troike, 2012). From a cognitive perspective, learning a language is an exclusively mental process in which learners actively figure out the rules of how a language is used in context by observing real-life situations (Suharno, 2010). Yet, cognition is not the only element that learners use to make assumptions about a language (Saville-Troike, 2012); and cognitivists tend to overlook social processes (Liang, 2013). For these reasons, sociocultural theory recognises the interaction of cognitive, psychological, and social characteristics in SLA, taking into account "the complex interaction between the individual acting with mediational means and their sociocultural contexts" (Swain \& Deters, 2007, p. 821). Accordingly, like a great deal of classroom research, this study adopts a sociocultural theory approach.

Since interaction facilitates the learning of target language functions and structures, the interaction hypothesis lays a significant foundation for the communicative language teaching approach (Cummins \& Davison, 2007). Moreover, communicative language teaching is a powerful approach that helps develop a learner's capacity for interaction in English in many EFL teaching and learning contexts (Rahman, 2017). Thus, communicative language teaching further develops SLA theory recognising that interaction is necessary for language development. Similarly, sociocultural approaches also "pay close attention to the settings and participants in interactions" (Foster \& Ohta, 2005, p. 403). Therefore, sociocultural theory, 
interaction hypothesis, and communicative language teaching are interconnected and together underpin this thesis.

\subsubsection{Sociocultural Theory}

Sociocultural theory (SCT) originated from Lev Vygotsky's work on child development in the 1910s. Vygotsky (1978) claims that it is through "social interaction and the transformation of practical activity" (p. 24) that knowledge is constructed and learning occurs. This implies that "individuals and environments mutually constitute one another and persons are not considered to be separable from the environments and interactions through which language development occurs" (Foster \& Ohta, 2005, p. 403). These tenets of Vygotsky's SCT have been developed and widely applied in the field of L2 and FL teaching in recent years. The most fundamental contribution of SCT is that it introduces "a richer understanding of the interpenetrated nature of individual development and social interaction for L2 learners, with dialogue not merely a vehicle of transmission of ideas from person to person[] but a mediator of mind" (Ohta, 2017, p. 59).

This thesis examines how teachers use English communication in classroom contexts which can be socially interactive, as well as the perceptions of both lecturers and students, which are influenced by cultural, social, educational, and cognitive factors. SCT offers a comprehensive framework to examine and discuss the multilayered and complex interrelations of factors that influence the language behaviour of the lecturers and the perceptions of both lecturers and students in my study. In the following paragraphs, inner speech, mediation, and scaffolding, and how these aspects of SCT can inform L2 research and teaching will be discussed.

To begin with, children first talk to others before they talk to themselves; thus, their speech passes from external speech to private and inner speech (Vygotsky, 1978). External speech is inherently social and occurs between people while private and inner speech are directed at oneself as an internalised and tacit communication and is related to thinking and to language processing. In terms of teaching, teachers act as language models for language activities, which are explained or presented in a socially interactive setting in class, and thereby offer examples of language use for students to follow. Also, teachers should correct students' ineffective language performances in order to ensure that they learn and use positive and effective speech directed to themselves (Werani, 2018). Teachers' language models and correction in the classroom help develop students' inner speech. Emotion plays a decisive role to ensure the transformation from external speech to inner speech (Swain, 2013). Interpersonal 
communication is also fundamental for moving from the unknown to the known state of knowledge (Lantolf \& Thorne, 2007), which I refer to as the external (unknown state) and inner speech (known state) of SCT.

Inner speech refers to verbal thought as a continuation of the child's egocentric speech which is not expressed out loud (Murphey, 1990). It is an important notion in Vygotsky's (1978) SCT. Although he did not explicitly relate inner speech to the learning of other languages, he addressed the issue by recognising that "the question of multilingualism and its possible (positive or negative) effects on mental and speech development was still not completely and satisfactorily resolved at his time and had to be looked at in all its complexity by further research" (Guerrero, 2018, p. 153). Vygotsky's (1978) concept of inner speech has inspired numerous studies on both children's and adolescents' L2 inner speech as an L2 learning mechanism. Saville-Troike (1988) studied the English communication strategies used by school children who speak Chinese, Japanese, and Korean as L1. The children employed L2 inner speech strategies such as close repetition of others' utterances and rehearsal of language prior to actual production. She suggests that the children's inner speech strategies were "related not only to the children's level of cognitive development and the difficulty of the learning task, but also to their social orientation and learning style" and the "L2 inner speech may have contributed to the L2 acquisition” (p. 586). DiCamilla and Antón (2004) analysed functions of inner speech that occurred during the collaborative interaction of L2 Spanish learner pairs. Results showed that inner speech mediated the focusing attention on relevant features of the task and created psychological distance from challenges encountered during task performances. Those two functions of private speech were interpreted as strategic means of gaining control of the task. Stafford (2012) investigated how inner speech was used by nine beginning L2 learners of Latin to complement different kinds of computer-based learning support. The study concluded that, from the early stage of learning, L2 learners use private speech to self-regulate during independent completion of at least some learning tasks and this may benefit L2 development.

The above discussions of inner speech have led to my argument that L2 inner speech plays a significant role in learners' L2 development in that it can enhance L2 attention and facilitate L2 cognition. Additionally, speech strategies and positive emotion or interpersonal communication are helpful to learners' L2 inner speech.

L2-oriented studies have revealed four important kinds of mediators. Firstly, language of all forms "is the most pervasive and powerful cultural artefact that humans possess to mediate 
their connection to the world, to each other, and to themselves" (Lantolf et al., 2014, p. 210). Thus, EFL teachers' classroom languages, both L1 and L2 and either in verbal or nonverbal forms, function as mediational tools for students' L2 learning. Secondly, Vygotsky's (1978) emphasis on the role of signs and symbols in transforming individuals' thinking implies the mediational functions of materials such as teaching facilities, textbooks, or other visual-aided tools. Thirdly, SCT also considers the role that others play in a person's language development. Thus, Vygotsky uses the example of a child pointing at an object which is out of reach, who then calls for other, more capable people, to act as a mediator and pass the object. When transferred to the L2 classroom setting, the teachers and more advanced learners function as mediators. Thus, two-way dialogues in L2 (between learner and teacher or learner and more capable learners) mediate the L2 learning process, allowing learners to construct their knowledge and develop skills in a way that serves their understanding of the actual application of the language. Lastly, Vygostky's (1978) emphasis on the importance of creating a supportive and motivating environment for learners in which their levels, needs, and interests are supported can be equated with the use of psychological mediation tools (Kozulin, 1998). The importance of emotions has been highlighted by others, who have found that emotions and learning "are linked and united in a complex process of internalisation over time" and thus emotions "come to mediate learning in current, local, contexts" (Swain, 2013, p. 205).

Along with mediation, the concept of scaffolding is also important in SCT. In the field of SLA, scaffolding involves providing linguistic support for learners and gradually reducing the support when they become more independent (Schumm \& Arguelles, 2006). Learners have the potential to move from their actual to their potential development level via scaffolding which occurs during their interaction with more experienced and more competent people (Khaliliaqdam, 2014; Lantolf et al., 2014). Furthermore, recent studies (e.g. Li \& Zang, 2020; Selvaraj et al., 2020; Zhang, 2019) have highlighted the positive effects of teacher strategic and instructional scaffolding in language classes. Accordingly, scaffolding practices play essential roles in EFL teaching, "in that learners at different English language proficiency levels need appropriate accommodations contingent on their developmental stages in order to explore and understand the content in a more accessible way" (Huang, 2020, p. 7).

Sociocultural theory has influenced many studies within L2 teaching and learning. One contribution of SCT in research is that it can explain a natural phenomenon by providing "a set of interrelated constructs, definitions, and propositions that present a systematic view of the phenomena by specifying relations among variables with the purpose of explaining the natural 
phenomenon" (Creswell, 2009, p. 51). SCT understands the connection between mental development and learning and takes into account not only "the contextual specifics but also the process over time", thereby capturing the "breadth and depth that encourages much of the story as possible" (Swain et al., 2015, p. xiii). The main contribution of this theory in L2 education is the attention it draws to "the cultural and social dimensions of language, thus changing the role of the teacher and the goal of and strategies for L2 learning" (Dixon et al., 2012, p. 35). Due to those reasons, I believe that SCT can provide a useful framework for understanding Vietnamese EFL lecturers' classroom English communication.

\subsubsection{Interaction Hypothesis}

The interaction hypothesis was formulated by Long in the 1980s and it has laid an important foundation for contemporary language teaching approaches (Cummins \& Davison, 2007; Mackey \& Polio, 2009). This hypothesis integrates the roles of comprehensible input, opportunities for output performances, and meaning negotiation during interaction for creating effective L2/FL acquisition. Since input itself is not as helpful as interactionally modified input in facilitating language teaching and learning, "the interaction hypothesis plays a central role in learning through conversational interaction on improving language learners' speaking skills" (Namaziandost \& Nasri, 2019, p. 221). The focus of the hypothesis on the role of interaction as a means to learn makes the interaction hypothesis central to the theoretical foundation of my study into classroom English communication. In the rest of this section, I will present the three key aspects of the interaction hypothesis and how they relate to classroom communication and L2/FL learning/teaching.

Firstly, the interaction hypothesis strengthens the role that comprehensible input plays in L2 acquisition in that comprehensible input (Krashen, 1980) is a characteristic of successful acquisition. Experiencing greater amounts of comprehensible input causes faster language acquisition, and lacking access to it results in little or no acquisition (Long, 1983). The interaction hypothesis proposes three ways to make input more comprehensible. Simplification during an interaction makes input more comprehensible and thereby promotes acquisition (Lightbown \& Spada, 1999). Modifications made by interlocutors during conversational interactions are an essential means of making input comprehensible. Recent studies (e.g. Namaziandost et al., 2019; Wang \& Castro, 2010) argue that interactionally modified input is more effective in enhancing comprehension and lexical acquisition than input provided with no interactional modification. Also, the use of extra linguistic content such as gestures and movements can also make input more comprehensible. A more competent speaker (e.g. 
teacher) has a central role in making input comprehensible, improving learner attention, and fostering learner input (Walsh, 2011). Thus, teachers' extensive use of classroom English and communication strategies to make English more accessible and comprehensible to learners is essential to English learning.

Secondly, the interaction hypothesis emphasises the role of meaning negotiation in providing learners with positive evidence, negative evidence, and opportunities for modified output (Long, 1996). Meaning negotiation is not just regarded as enabling learners to obtain comprehensible input (called positive evidence); it also helps learners gain information about what is ungrammatical as well as their linguistic gaps (called negative evidence) (Wei, 2012). Negotiation can serve as an attention-drawing device which "focus[es] learners" attention on potentially troublesome parts of their discourse, providing them with information that can then open the door to interlanguage modification" (Fang, 2010, p. 14). Meaning negotiation "is one of a range of conversational processes that facilitate SLA as learners work to understand and express meaning in the L2" (Foster \& Ohta, 2005 p. 402). Therefore, L2 learners should be encouraged to negotiate meaning as much as possible during L2 interactions (Hartono, 2017). Teachers can promote negotiation of meaning in an English classroom by creating an interactive learning environment with appropriate communication tasks (Lengluan, 2008). Also, teachers' interactional strategies such as comprehension checks, confirmation checks, and clarification requests are argued to play important roles in enhancing meaning negotiation of learners and thus facilitating their SLA.

The third and last key concept of the interaction hypothesis posits that meaning negotiation "facilitates acquisition because it connects input, internal learner capabilities, particularly selective attention, and output in productive ways" (Long, 1996, p. 452). In fact, when input enhancement is incorporated into output activities, learners can effectively form their hypothesis of the target rule (Song \& Dai, 2009). Compared to input in SLA, output "pushes learners to process language more deeply - with more mental effort than does input" (Swain, 2000, p. 99). It was found that output-based instruction can enhance EFL learners' willingness to communicate more than input-based instruction (Roohani et al., 2017). This is supported by Kim (2014), whose study indicated that output-based tasks such as writing have been evidenced to help EFL learners have better oral performances (e.g. speaking faster with fewer pauses). Therefore, lecturers' strategies to encourage learners' engagements and responses to their L2 speech are argued to help the learners identify their L2 gaps and better acquire the language. 
This is where interaction hypothesis overlaps with sociocultural theory as both propose that dialogic and conversational situations in L2 can enhance the L2 learning process.

The above discussion has shown that the combination of comprehensible input, meaning negotiation, and productive output facilitate the process of language acquisition. In addition, communication strategies play important roles in making input more comprehensible, negotiation of meaning more effective, and output more productive. Through the use of communication strategies, L2 teachers and learners can improve their oral proficiency and communication skills (Díaz Larenas, 2011).

The interaction hypothesis, together with the sociocultural theory, inform the theoretical framework for my research into classroom English communication since both theories conceptualise the significant role of social interaction in L2 development, which is central to my study. The most natural way to acquire an L2 in classrooms is through authentic L2 communication and through as much interaction with classmates and teachers as possible. Sociocultural theory and the interaction hypothesis are considered to lay the foundation for the communicative language teaching approach as well, which is described in the following section.

\subsubsection{Communicative Language Teaching}

Communicative language teaching (CLT) is an approach to language teaching that was born out of sociolinguists' beliefs that it is not knowledge of vocabulary and grammar rules that are central for language learning but the ability to use the language appropriately in different contexts. CLT has come to be the most generally accepted approach in language teaching, possibly because the desired outcome of CLT is that learners can communicate successfully in the target language in real situations, instead of knowing the rules governing the language (Knight, 2001). This approach puts an emphasis on learners' ability "to use and produce the language effectively, improve their basic communicative skills, and engage in effective communication in the target language" (Owen et al., 2019, p. 468). Accordingly, CLT classrooms involve interaction and communication offering learners the opportunity to practice their skills and assume different interactional roles through the use of communicative activities and tasks (Albahri et al., 2018). The CLT approach "ensures learners get enough exposure to the target language" (Haryono, 2019, p. 48). Due to this emphasis on L2 communication, the use of learners' L1 is discouraged or even prohibited in many EFL classrooms.

CLT has been applied as an effective approach in many English as a second and foreign language teaching contexts. In educational settings where English is taught as a second 
language, CLT seems to be more advantageous. This is because learners need to use English to communicate outside the classrooms, which creates a sense of positive communicative pressure among the learners to practice communicating in English inside the class. However, this advantage does not apply in EFL teaching contexts since learners have neither the pressure nor the opportunities to use English after they step outside classrooms. Moreover, EFL teachers' ability in speaking or communicating in English is often not sufficient to conduct the communicative lessons necessarily involved in CLT ( $\mathrm{Li}, 2001)$. In EFL classrooms, it is suggested that interactive competence and meaningful communication should be given a greater priority than the accuracy of the language structures in CLT practices (Christianto, 2019). EFL learners can be competent in communicating in the target language if they make the effort to develop their proficiency through meaningful and genuine practices (Pham, 2007), which need to be about everyday life situations that entail communication (Alwazir \& Shukri, 2017).

CLT in Asian EFL Teaching Contexts. CLT spread to Asia in the 1970s with its premise being to "develop communicative competence through meaningful interactions" (Butler, 2017, p. 329). Benefits and effectiveness of CLT in EFL teaching settings have been identified. First and foremost, it encourages students to speak the target language and participate actively in classroom activities, which was found to enhance students' confidence and competence (Adamou, 2017). For instance, Al-Garni and Almuhammadi (2019) conducted a quasi-experimental study in Saudi Arabia. The experimental group was taught for three weeks (1,5 hours per week) using interviewing, problem-solving, and role-playing as communicative activities, while the control group was taught using traditional methods. The findings from preand post-tests for IELTS speaking showed that the experimental group scored much higher than the control group, providing evidence for a positive effect of CLT teaching practices on improving students' speaking skill. CLT was also found to increase learners' interest, raise learners' awareness of the learned language, and provide exposure to the real target language (Gorbani, 2019; Richards, 2006). Research further suggests that CLT fosters a sub-conscious process of acquiring a second language and learners' communicative competence (Gurunathan \& Geethanjali, 2016; Kwon, 2017). Finally, the CLT approach also seems to help establish a positive relationship between teacher and students (Chang \& Goswami, 2011; Rahman, 2017). Considering the benefits of CLT, it has been strongly recommended to be applied in EFL countries (Ariatna, 2016; Athawadi, 2019; Jahanzaib \& Zeeshan, 2017).

Despite the recommendations above, a number of studies have demonstrated disadvantages as well as complications that EFL teachers have encountered when implementing CLT. For 
example, in the context of China, Luo and Garner (2017) assert that cultural difficulties should be overcome if this approach is to be successfully adopted. This is because in the Confucian culture of China, learners are oriented towards receiving knowledge passively from the teacher rather than to being actively engaged in constructing knowledge for themselves. In Indonesia, limited teacher expertise and teaching time, structure-based syllabus, grammar-based designs of textbooks, and limited communicative language testing impede the adoption of CLT (Rahman, 2017; Yanti, 2019). In Bangladesh, lack of effective and appropriate teacher training, teachers' inclination to stick to traditional approaches, teacher-centred classrooms, focusing on exams instead of communication, and logistic limitations such as large groups of students are inhibitors to the implementation of CLT and classroom change (Hassan, 2014; Mahmadun Nuby et al., 2020). In Pakistan, the CLT approach is recognised to be applicable but hindered as contextual problems such as large classes and overuse of traditional teaching methods which limit students' opportunities to engage in interactive activities with their peers (Panhwar et al., 2017). Taken together, traditional teaching and learning habits, grammar-focused testing, lack of training, and class size are the four most pressing barriers to the implementation of CLT in Asian EFL teaching.

CLT in the Vietnamese EFL Teaching Context. In Vietnam, CLT has only recently gained popularity as Vietnamese people seek communicative outcomes when learning English in order to better integrate within the wider world (Kieu, 2010). Accordingly, a guiding programme for adopting a CLT approach has been implemented nationwide since 2010. This programme states that the methods of teaching should conform to three guiding principles relating to: communication, tasks, and meaningfulness (Vietnamese Government, 2010). Meanwhile, the Ministry of Education and Training has organised CLT workshops, seminars, and conferences to support EFL teachers to better integrate this approach into their teaching practices. Studies evaluating those programmes (e.g. Mai \& Iwashita, 2012; Pham, 2007) have indicated that CLT can assist Vietnamese teachers to encourage students' involvement in communicative tasks that simultaneously preserve Vietnamese culture. CLT is considered to be the most effective method due to its advantages, feasibility, and its ability to accommodate Vietnamese cultural learnings. Overall, the CLT approach is believed to foster Vietnamese learners to communicate better and to meet the requirements of globalisation (Ha, 2019).

However, as with the situation of other Asian EFL teaching contexts, CLT has also faced some difficulties when being applied in the context of English teaching in Vietnam. Firstly, no matter how much or how little training they receive about communicative language teaching, 
Vietnamese teachers remain very focused on grammar teaching (Le, 2011). In fact, many teachers still employ the grammar translation method because this is easy and makes them feel more confident while teaching (Do, 2013; Mai \& Iwashita, 2012). Secondly, it seems that, while Vietnamese teachers of English hold strong positive beliefs about the value of CLT, they have difficulties translating those beliefs into their classroom practice because of students' low motivation and limited English proficiency (Le, 2020; Nguyen, 2019). Other reasons for the failure of CLT implementation include teachers' own limited capabilities in English and lack of confidence in CLT implementation (Nguyen, 2013). Overcrowded classrooms, the fact that English is not used in daily life in the Vietnamese context (Mai, 2017), a preference for testing language knowledge rather than communication skills (Pham, 2004), and insufficient time available for teaching ( Nguyen, 2019; Nguyen et al., 2015) were also identified as barriers.

\subsubsection{How do the Sociocultural Theory, Interaction Hypothesis, and Communicative}

\section{Language Teaching Approach Frame this Study?}

This thesis proposes an interwoven relationship between sociocultural theory, the interaction hypothesis, and CLT in affecting EFL teaching and learning. Specifically, this study incorporates sociocultural theory in that engagement of a teacher in a sociocultural context may affect his/her language use and perceptions which can impact on all aspects of English teaching (Cross, 2010). Also, English learning can be facilitated by socioculturally mediated institutional and cultural artefacts (Lantolf, 2000) such as the physical set up of the classroom, methods of error feedback, and dialogues. The communicative language teaching approach is further complemented by the use of the sociocultural concepts (Farsia, 2016), since the CLT approach also considers different levels of interaction and communication where learners have opportunities to practice their skills in various ways and by taking different roles.

The interaction hypothesis underpins the thesis in the assumption that students will better acquire the English language if their teachers make suitable modifications in their spoken English, an important source of input (Gupta, 2004), Also, it is assumed that a teacher's communication strategies better engage students and encourage meaning negotiation among the students, ensure they receive more comprehensible input, and produce better output, which helps them acquire better English.

Hence, sociocultural theory, interaction hypothesis, and the communicative language teaching approach delineate the various areas that need to be considered in the analysis to fully answer 
the research questions. They even provide categories, for instance for communication strategies, that can be applied to the data.

\subsection{Communication in L2/FL Teaching and Learning}

Besides having characteristics similar to other communication situations, classroom communication happens to transfer knowledge between a teacher and students (or among students) during the process of teaching and learning, as well as serving other purposes such as making a good class atmosphere or establishing rapport in the class. In L2/FL classroom contexts, participants can improve the success of their utterances in L2 communication by using communication strategies (Jamshidnejad, 2011). I begin this section by providing definitions of key concepts, followed by a discussion of communication strategy taxonomies, which leads to a description of the communication strategy taxonomy that will be used in this study and empirical research on teachers' English communication in L2/FL teaching contexts.

\subsubsection{Definitions of Key Concepts}

Classroom Communication. Classroom communication has been defined as:

\footnotetext{
"interactions during the lesson which can take place verbally, non-verbally and in writing and can influence the outcome of the learning process. Thus, the success of every child to acquire quality education can depend to a large extent on the way the teacher manages communication strategies and techniques with the learners in the classroom" (Len, 2018, p. 536).

"a two-way interaction process between teachers and students, which can maximise the activation of students' initiative and improve their knowledge application ability" (Yang, 2020, p. 1046).

"a complex, multifaceted and multi-channel transfer of information between two entities (individuals or groups) that simultaneously and successively assume the roles of receivers and transmitters in the context of the instructive-educational process" (Stefan \& Popescu, 2020, p.
} 62)

As can be seen from the definitions above, classroom communication is, like other communication situations, a process of meaning exchanges and construction. However, communication within classroom contexts aims at enhancing students' knowledge, skills, and development rather than just exchanging information or promoting comprehension as daily communication. In order to do this, teachers play the central role and have to communicate with their students in different ways and try techniques and strategies. Moreover, classroom communication in EFL classes is not necessarily similar to that happening in normal native 
language classes where teachers and learners use only their first language. Firstly, apart from English - the learnt language, the first language can be also used as well. Secondly, there can be a big inconsistency in the language proficiency levels between teachers and learners, which can challenge or hinder the English communication between them. Furthermore, classroom English in EFL classes is actually a kind of English for specific purposes (e.g. academic or occupational); it is the kind of language the teacher needs to use in the classroom for different purposes (e.g. academic or occupational); it is the kind of language the teacher needs to use in the classroom for different purposes (e.g. giving instructions, managing students' behaviours) (Freeman et al., 2015). Therefore, to communicate more effectively in EFL classes, teachers are required to take different roles and have relevant skills and knowledge.

From the above definitions and features of EFL classroom contexts, I use the term classroom communication to refer to the use of both verbal and nonverbal language in teaching and communicating with students during the lessons to enhance students' English knowledge and skills as well as for other pedagogical purposes, and classroom communication can be enhanced thanks to the teacher's communication strategies and skills.

Communication Strategies. In the context of SLA, the concept of communication strategies (CSs) has been defined differently depending on which theoretical frameworks have been applied. From an interactional perspective, CSs refer to a "mutual attempt of two interlocutors to agree on a meaning in situations where requisite meaning structures do not seem to be shared" (Faerch \& Kasper, 1983, p. 65). In other words, CSs are conceptualised as "attempts to bridge the gap between the linguistic knowledge of the second language learner, and the linguistic knowledge of the target language interlocutor in real communication situations" (Tarone, 1983, p. 65). During a communication process, L2 learners may not be able to discuss or convey their thoughts effectively to the listeners due to a lack of L2 linguistic proficiency skills. Communication strategies are therefore key to solving such problems. From a psycholinguistic approach, CSs are referred to as cognitive processes which involve perception and production in a target language. Researchers at Nijmegen University described CSs as "differences in processes of referential communication rather than differences in the linguistic formal strategic utterances or differences between referents or differences in context" (Bongaerts \& Poulisse, 1989, p. 255). They assumed that, both 1) the cognitive process which underlies the choice of a strategy and 2) the factors which influence the process of such a selection, are essential. Yang and Gai (2010) regarded CSs as techniques people use when facing communicative problems or trying to achieve communicative goals. The use of CSs is 
interactively affected by factors such as “a learner's level of language proficiency, a learner's personality and communicative experience, and his/her attitude towards a particular strategy, nature of task, problem source, or communication situations" (p. 74).

Despite different approaches, based on the definitions provided above, it is clear that most L2 CSs respond to 'problematicity' and 'consciousness' as two elements. The term 'problematicity' refers to "the idea that strategies are used only when a speaker perceives that there is a problem which may interrupt communication" (Bialystok, 1990, p. 3). 'Consciousness' is usually interpreted as the awareness of a problem and/or strategic language use, intentionality, and control (Dörnyei \& Scott, 1997). In fact, researchers (e.g. Cohen, 1998; Dörnyei \& Scott, 1997; Muhlisin \& Widyanto, 2019) perceived strategy application as a conscious process, claiming that speakers use communication strategies as conscious attempts to deal with communication-related problems of which they were aware. Other researchers (e.g. Gass \& Selinker, 1994; Kárpáti, 2019), however, asserted that some speakers apply communication strategies automatically, meaning that it is controlled by their sub- and unconsciousness. For the purposes of this study, I hold that teachers' CSs in L2 classroom interactions are used both consciously and unconsciously for two reasons. Firstly, teachers used to be L2 learners and users, underwent professional training, and had experiences in communication, which can help them intentionally use certain L2 communication strategies for pedagogical purposes. Meanwhile, since L2 teachers already have communicative competence, they are also able to employ L2 strategies unconsciously to deal with situations which are new, unfamiliar, or simply unaccounted for in their teaching plans.

\subsubsection{Communication Strategy Taxonomies}

Dörnyei and Scott's Taxonomy (1997). Over the last three decades, numerous communication strategy typologies have been proposed in the research literature, which were strongly influenced by linguists' approaches to language analysis. The most important among those may be Dörnyei and Scott (1997), who reviewed nine different taxonomies (e.g. Bialystok’s (1990); Færch \& Kasper's (1983); Tarone's (1977)) and discovered significant similarities among them. They subsequently integrated the different approaches and suggested the new taxonomy based on their own previous version proposed in 1995, with a total of 33 strategies (see Appendix N, for full descriptions and examples of each strategy).

Based on how CSs contribute to solve these problems, Dörnyei and Scott (1997) classified their taxonomy into direct, indirect, and interactional strategies, and under each of these they list 
sub-categorised strategies. Direct strategies involve speakers' attempts to find alternative means (e.g. circumlocution compensating for the lack of a word) to make themselves understood. Indirect strategies refer to attempts to "facilitate the conveyance of meaning indirectly by creating the conditions for achieving mutual understanding" (p. 198). Interactional strategies enable participants to "carry out trouble-shooting exchanges cooperatively, and therefore mutual understanding is a function of the successful execution of both pair parts of the exchange" (p. 199). Strategies to deal with the problems facing performances and relating to the deficiencies in linguistic resources were found in all the three major strategies. Strategies to cope with processing time pressure-related problems were put in the indirect category. An overview of Dörnyei and Scott's taxonomy (1997) is summarised in the table below.

\section{Table 2.1}

Dörnyei and Scott's Taxonomy (1997, p.188-194)

\begin{tabular}{|c|c|c|}
\hline Direct Strategies & Interactional strategies & Indirect strategies \\
\hline $\begin{array}{l}\text { Resource deficit-related strategies } \\
\text { *Message abandonment } \\
\text { *Message reduction } \\
\text { *Message replacement } \\
\text { *Circumlocution } \\
\text { *Approximation } \\
\text { *Use of all-purpose words } \\
\text { *Word-coinage } \\
\text { *Restructuring } \\
\text { *Literal translation } \\
\text { *Foreignising } \\
\text { *Code-switching } \\
\text { *Use of similar sounding words } \\
\text { *Mumbling } \\
\text { *Omission } \\
\text { *Retrieval } \\
\text { *Over-explicitness } \\
\text { *Mime } \\
\text { Own-performance problem-related } \\
\text { strategies } \\
\text { *Self-rephrasing } \\
\text { *Self-repair } \\
\text { Other-performance problem-related } \\
\text { strategies } \\
\text { *Other-repair }\end{array}$ & $\begin{array}{l}\text { Resource deficit-related strategies } \\
\text { *Direct/indirect appeals for help } \\
\text { Own-performance problem-related } \\
\text { strategies } \\
\text { *Comprehensive check } \\
\text { *Own-accuracy check } \\
\text { Other-performance problem-related } \\
\text { strategies } \\
\text { *Asking for repetition } \\
\text { *Asking for clarification } \\
\text { *Asking for confirmation } \\
\text { *Guessing } \\
\text { *Expressing non-understanding } \\
\text { *Interpretive summary } \\
\text { *Responses (repeat/ repair/ rephrase/ } \\
\text { expand/ confirm) }\end{array}$ & $\begin{array}{l}\text { Processing time } \\
\text { pressure-related } \\
\text { strategies } \\
\text { *Use of fillers } \\
\text { *Self-repetition/ } \\
\text { Other- repetition } \\
\text { Own-performance } \\
\text { problem-related } \\
\text { strategies } \\
\text { *Verbal strategy } \\
\text { markers } \\
\text { Other-performance } \\
\text { problem-related } \\
\text { strategies } \\
\text { *Feigning } \\
\text { understanding }\end{array}$ \\
\hline
\end{tabular}

The CS typology given by Dörnyei and Scott (1997) can be considered to be the most useful because it integrates different, previously established taxonomies. Moreover, this taxonomy lists many strategies that are relevant to my research aims. Hence, it has been chosen as the main foundation for the taxonomy employed in this study. 
This Study's Proposed Communication Strategy Taxonomies. As mentioned previously, this study examines CSs from the stance of teachers, in the context of a high-level interlocutor communicating with much lower level learners in L2 when they both share L1, which is different from the contexts and subjects of most current taxonomies. Therefore, I have composed my own context-based CS taxonomy, basing mainly on the model of Dörnyei and Scott (1997) but also drawing on other sources. The basis of the taxonomy proposed in this study is a consideration of the characteristics of teachers' talk and the purposes the CSs are aimed at. An overview of this study's CSs taxonomy is provided in the following table and an elaboration follows.

\section{Table 2.2}

This Study's Proposed Taxonomy

\begin{tabular}{|c|c|c|}
\hline Strategies & Adopted from & Definition/ Description by linguists \\
\hline $\begin{array}{l}\text { Code- } \\
\text { switching }\end{array}$ & $\begin{array}{l}\text { Faerch and Kasper (1983) } \\
\text { Willem (1987) } \\
\text { Dörnyei and Scott (1997) }\end{array}$ & $\begin{array}{l}\text { Including L1 words with L1 pronunciation in } \\
\text { L2 speech; this may involve stretches of } \\
\text { discourse ranging from single words to } \\
\text { whole chunks and even complete turns } \\
\text { (Dörnyei \& Scott, 1997, p. 189). }\end{array}$ \\
\hline Translation & $\begin{array}{l}\text { Tarone (1980) } \\
\text { Willem (1987) } \\
\text { Dörnyei and Scott (1997) }\end{array}$ & $\begin{array}{l}\text { Translating a lexical item, an idiom, a } \\
\text { compound word or structure from L1 to L2 } \\
\text { (Dörnyei \& Scott, 1997, p. 189). }\end{array}$ \\
\hline Nonverbal & $\begin{array}{l}\text { Dörnyei and Scott (1997) (Embodied in } \\
\text { Mime) }\end{array}$ & $\begin{array}{l}\text { Describing whole concepts nonverbally or } \\
\text { accompanying a verbal strategy with a visual } \\
\text { illustration (Dörnyei \& Scott, 1997, p. 190). } \\
\text { Directly linked to learners' use of body } \\
\text { language such as eye contact, gestures, and } \\
\text { facial expressions to achieve communication } \\
\text { (Nakatani, 2006, p. 157). }\end{array}$ \\
\hline Interactional & $\begin{array}{l}\text { Dörnyei and Scott (1997) } \\
\text { (Embodied in mumbling; omission; } \\
\text { retrieval; over-explicitness; comprehensive } \\
\text { check; own-accuracy check; asking for } \\
\text { repetition/ clarification/ confirmation; } \\
\text { expressing non-understanding; interpretive } \\
\text { summary; response (repeat/ repair/ } \\
\text { rephrase/ expand/ confirm); use of fillers; } \\
\text { and verbal strategy markers) }\end{array}$ & $\begin{array}{l}\text { Involving a third approach, whereby the } \\
\text { participants carry out trouble-shooting and } \\
\text { therefore mutual understanding is a function } \\
\text { of the successful execution of both pair parts } \\
\text { of the exchange (Dörnyei \& Scott, 1997, p. } \\
\text { 199). }\end{array}$ \\
\hline Self-repetition & Dörnyei and Scott (1997) & $\begin{array}{l}\text { Repeating a word or a string of words } \\
\text { immediately after they were said (Dörnyei \& } \\
\text { Scott, 1997, p. 190). }\end{array}$ \\
\hline Simplification & $\begin{array}{l}\text { Faerch and Kasper (1983); Willem (1987) } \\
\text { (Embodied in Reduction strategy) } \\
\text { Poulisse (1993) (Embodied in Substitution } \\
\text { strategy) } \\
\text { Dörnyei and Scott (1997) } \\
\text { (Embodied in message } \\
\text { abandonment/reduction/replacement; } \\
\text { circumlocution; approximation; use of all- } \\
\text { purpose words; use of similar sounding } \\
\text { words; word-coinage; restructuring; } \\
\text { foreignising; and self-rephrasing) }\end{array}$ & $\begin{array}{l}\text { A proposed strategy in this study. Its } \\
\text { definition will be provided later. }\end{array}$ \\
\hline
\end{tabular}


I decided not to group strategies into the main three categories (direct, indirect and interactional) as Dörnyei and Scott (1997) did because I did not look CSs from the manner of problem management but the purposes of lecturers' attempts to use classroom communication strategies. Strategies were also not categorised into the preconceived categories of problem causes identified by Dörnyei and Scott (1997) (e.g. resource deficit-related; performance problem-related; or processing time pressure-related) because these seem not to be significant problems that L2 teachers encounter when communicating with students; the biggest problem facing teachers is how to make their students better comprehend their message. Moreover, based on the definitions and explanations provided by Dörnyei and Scott (1997), I decided not to include 'appeals for help' and 'guessing' strategies because they do not seem relevant for the current setting of teachers speaking L2 to students in L2 classes. The 'feigning understanding' strategy is excluded as well since it would be impossible to identify its use via observation.

The categories of 'code-switching', 'translation', 'nonverbal', 'interactional', and 'selfrepetition' are directly adopted from different typologies. I developed the 'simplification' category by integrating different elements from Dörnyei and Scott's (1997) taxonomy which share characteristics and aim at similar purposes to make the 'simplification' strategy which is more relevant to the research aims. The simplification strategy has not been explicitly included in the available CSs taxonomies. This may be due to the fact that CSs were examined as a tool which aimed at helping L2 learners to communicate successfully when having productionrelated problems. As for L2 teachers who are communicating with L2 students, the main reason for using those strategies (e.g. substitution, circumlocution, or paraphrase) may not be their own linguistic shortages, but to make their L2 speech simpler and more accessible for their students. Reasons for the choices and definitions of the strategies, including discussions of how they match the research context and the study aims, are described below.

Code-switching from L2 to L1 is a common phenomenon in L2/FL classes when teachers and learners share an L1. In Tarone's (1977) original taxonomy, this phenomenon is called 'language switch', which was later adopted by Bialystok (1990). Willems (1987) referred to this strategy as 'borrowing'. However, most available CSs taxonomies, especially the one of Dörnyei and Scott (1997), labelled this phenomenon code-switching. In this research, I define code-switching as the insertion of L1 words/chunks/turns in L2 speech for communicative purposes. 
I added the category of translation to the CSs taxonomy used for this study because it is another CSs commonly mentioned by linguists. It should be clarified that translation here is not regarded as a teaching technique or activity in L2/FL classes, but as a communication strategy in L2 speech. As mentioned in the beginning of this section, the context in which CSs are examined in this study is different from the contexts for most previous CSs taxonomies since in this case, both the speaker and interlocutor share an L1. Moreover, when using L2 to teach and speak to students of a lower L2 proficiency who share the L1 with the teacher, translation to L1 seems to be a natural phenomenon in EFL classes. Hence, translation as a communication strategy used by teachers refers to translating L2 lexical and syntactic units into L1, not from L1 to L2 as defined by most contemporary scholars.

With regard to the application of nonverbal devices as communication strategies, mime was the most frequently used in early CSs taxonomies (e.g. Bialystok, 1990; Dörnyei \& Scott, 1997; Tarone, 1980). Later, Nakatani (2006) used the term 'nonverbal' strategies and concluded that these are used for coping with both speaking and listening problems during communicative tasks. When speaking English, a learner uses "eye contact in order to attract the attention of their listener" and "gestures or facial expressions to give hints and help the listener guess what they want to say" (Nakatani, 2006, p. 155). I support Nakatani's view and added 'nonverbal' as a CS in this study, defining it as the use of any nonverbal devices such as gestures, posture, mime, or facial expressions as supportive tools to help make sense or clarify lexical/ structural units in L2 verbal speech. The term mime was considered to be too restrictive as it refers to just one kind of nonverbal device. The category of 'nonverbal' strategies proposed for this study, is designed to capture a wide range of nonverbal strategies used by L2 teachers.

Because interaction plays a vital role in L2 learning, where it can serve to facilitate the exchange of information and prevent communication breakdowns (Ellis, 1990), the 'interactional' strategy was chosen to be an element of this study's CSs taxonomy. The CSs model of Dörnyei and Scott (1997) suggested ten minor strategies in their interactional strategy (see Table 2.1). As mentioned previously, 'appeals for help' and 'guessing' were excluded because these strategies are used to explicitly show speakers' failure to perform or comprehend native interlocutors, which is not applicable in the current case. Hence, the remaining eight interactional strategies, 'mumbling', 'omission', 'retrieval', 'over-explicitness' (in the direct type), 'use of fillers', and 'verbal strategy markers' (in their indirect category) were grouped to constitute the interactional strategies in this study. For the purposes of this research, interactional strategies are defined as techniques such as summarising what have been said; 
checking for the listener's comprehension by asking questions; communicating the intended message basing on an alternative plan; swallowing or muttering inaudibly a word; leaving a gap; retrieving a lexical item; or the use of pauses/fillers (e.g. well; you know; actually; okay) to keep the communication channel open, overcome their linguistic deficiencies/failure, and enhance students' attention and comprehension.

Although the first model of CSs was formed in 1977 by Tarone (1977), it was not until 1997 that 'repetition' was perceived to be a communication strategy and included in Dörnyei and Scott's taxonomy. This means that repetition is a comparatively new element in the system of CSs typologies in the literature. In their model, Dörnyei and Scott divided repetition into 'selfrepetition' and 'other-repetition', defining the former as "repeating a word or a string of words immediately after they were said" and the latter being "repeating something the interlocutor said to gain time" (p. 190). This study adopted the self-repetition type because the other one aims at gaining time, which is likely to be irrelevant since this study mainly focus on teachers' speech who are not likely to use this strategy. In this study, self-repetition refers to a speaker's repetition of any parts of their L2/FL speech for purposes such as allowing time for the listener to decode the meaning or enhancing his/her L2/FL memorisation process.

'Simplification' occurs when a speaker thinks that, by using this strategy, he/she will be best understood by the addressee. Since parts of a teacher's L2/FL speech, such as vocabulary or structures, may pose challenges for students to understand, one of the ways for the teacher to tackle this problem is to modify the lexical and linguistic units in his/her speech so that they are more familiar or easier for students to gauge their meanings. I categorised these modification processes as simplification strategies.

My idea of the simplification strategy originated from different strategies which all serve to substitute the original linguistic unit with a new one which is simpler. The current simplification strategy is thus based on the concepts of 'reduction' (Faerch \& Kasper, 1983; Willem, 1987); 'substitution' (Poulisse, 1993); and some previously-mentioned strategies in the taxonomy of Dörnyei and Scott (1997). By using the strategies of 'message abandonment/reduction/replacement'; 'circumlocution'; 'approximation'; 'use of all-purpose words'; 'use of similar sounding words'; 'word-coinage'; 'restructuring'; 'foreignising'; and 'self-rephrasing', a speaker wants to make it easier to express and/or more accessible to the interlocutor. For the case of an L2/FL teacher speaking English to students, those strategies aim at simplifying their speech; hence, they were integrated into the simplification strategy. 
Putting together all the suitable attributes of the strategies constituting the simplification strategy and referring them to the context of our research, I describe the concept of simplification strategy in this study as using techniques excluding the use of L1 or nonverbal means to simplify L2/FL speech at the point of, or after utterances.

As can be seen, most original CSs definitions were built from the stance of L2 learners who are likely to encounter a lot of problems that are usually caused by gaps in their linguistic system and hence utilise strategies to overcome "roadblocks to effective communication" (Tarone \& Swierzbin, 2009, p. 72). What is more, the interlocutors in the above CSs research normally do not always share a common L1 with the speaker, meaning that they had to try a wide range of techniques to express themselves. In this study, I examine CSs used by L2 English-speaking lecturers in the context of teaching English as a foreign language to nonEnglish major students who share a common L1, whose English competency may be rather limited, who may not be very motivated, and who may, therefore, feel burdened by the English language classroom (Afrin, 2016). The overarching term 'communication strategy' in this research has two typical features which are different from those of the available CSs research. Firstly, the problems that teachers face are not so much due to their lack of linguistic competency, meaning they have fewer difficulties in expressing themselves in front of their students, but in their effort to make the students better comprehend their utterances. Secondly, the teachers' CSs are pedagogical and affected by different factors, among which students play an important role.

Moreover, this current research is underpinned by the communicative language approach, sociocultural theory, and Interactional Hypothesis in English teaching. Therefore, considering these three different contexts and based on the reviewed literature and taxonomies of CSs, I propose the following definition of CSs:

CSs are verbal/nonverbal means, L1-based/L2-based techniques which are supportive to lecturers' L2 communication; and are both consciously and unconsciously used for the following purposes: helping students when the lecturers realise that students are encountering difficulties in comprehension; enhancing students' listening motivation; encouraging students' responses; or helping the lecturers overcome their inadequacies in relation to linguistic competency/performance. 


\subsubsection{Empirical Research in Teachers' English Communication and Strategies in EFL Teaching Context}

Investigations into teachers' classroom English in EFL classes has focused on three main topics: amount, features, and strategy use. Section 2.3 will report on research on the amount of English used in EFL classrooms. This section in turn reviews research on the features of teachers' English talk and teachers' English communication strategies.

Features of Teachers' English Talk. A number of researchers have described the characteristics of the language used by teachers of English. Using a descriptive observation method, Irmayani and Rachmajanti (2017) explored lexical features of teacher talk in EFL classrooms in Indonesia. Results revealed that teachers used simple language and vocabulary, with nouns and verbs being used most frequently. The authors suggest that the EFL classroom did not present students with an L2 environment marked by rich and varied lexical input because teachers rarely provided elaborations in L2 and offered few opportunities for learners to negotiate. Two studies by Nurpahmi (2017) and Sahlim (2018) in Indonesia reported on the functional characteristics of teachers' English talk, such as giving directions and instructions, clarifying, questioning, and encouraging. Each functional feature was employed to make the teaching process meaningful for the students' comprehension.

Modifications or adjustments in teachers' English talk when interacting with students have also been studied. For example, a study by Al-Ghamdi and Al-Bargi (2017) revealed that Saudi EFL teachers frequently used varied linguistic and interactional techniques to modify their talk. These techniques included simplifying vocabulary and structures, shortening sentences, slowing down speech, using emphatic stress, and repetition. Findings also suggested that teachers' modification strategies enhanced students' comprehension and developed classroom interaction. L2 speech modification has also been found at a phonological level. The results of Hamzah's (2019) study showed that Indonesian EFL teachers frequently used more extended pauses, spoke at a slower rate, and exaggerated articulation during English speaking in lessons. Hamzah (2019) proposed that these modifications were used in order to emphasise the presented material, provide comprehensible input, and model the target language for the students. Ünel and Mirioğlu (2015) studied eight EFL instructors at Çag University in Turkey, reporting that both native and non-native teachers of English made lexical modifications in their English talk when teaching elementary and pre-intermediate levels. Findings also indicated that these lexical modifications were responses to the proficiency level of the learners. 
The studies described above have shown EFL teachers' awareness of their English talk as an important L2 input for students and provided insights into how they try to modify their language use to make it more accessible to students. The linguistic choices teachers make in class are influenced by students' comprehension and meaning negotiation.

Teachers' English Communication Strategies. Research and studies on communication strategies have mostly focused on learners rather than teachers, which might be because the original CSs taxonomies were designed to capture L2 learners' approaches to communication breakdowns in order to help them manage these potentially stressful situations. Not many studies have examined teachers' communication strategies, particularly in the context of EFL teaching.

Teachers' English communication strategies in EFL teaching contexts has been examined with regard to what strategies teachers used during classroom interaction. For example, YaghoubiNotash and Karafkan (2015) investigated the functions of CSs employed by 16 EFL teachers when using English in a range of elementary and advanced levels settings at private institutes in Tabriz, Iran. The T-test results indicated a significant difference regarding the function of 'maintaining the flow of conversation' between elementary and advanced level teachers. Muhlisin and Widyanto (2019) conducted a discourse analysis of the language used by an English teacher in communication with first-grade students of Bonjeruk using the CSs model by Celce-Murcia et al. (1995). Results showed four communication strategies used by the teacher, with code-switching being used most frequently. Dyana (2020) conducted a qualitative research to find out the CSs used by an Indonesian EFL teacher using Celce-Murcia's taxonomy. Findings show five strategies and eleven sub-strategies applied by the teacher with filler being the most frequent while retrieval and clarification request being the least used strategies.

Studies have also investigated factors affecting teachers' choices and use of CSs. It has been suggested that the differences in teachers' proficiency level (Garcés \& Olivera, 2014; Rahmani Doqaruni, 2017), teaching experience (Boonkongsaen, 2018; Rahmani Doqaruni, 2017), and communicative goal (Garcés \& Olivera, 2014) result in different CSs selections. Teachers' cognition and language awareness have also been found to have an influence on their use of CSs. For example, Rahmani Doqaruni (2017) compared inexperienced and experienced groups of teachers at the university of Mashhad in Iran who had enrolled in an course on communication strategies. Observation and interview data indicated that experienced teachers used much fewer CSs than their novice counterparts, who employed the same types of CSs 
(e.g. circumlocution, miming) at a relatively high frequency. The author suggested that this was because experienced teachers had become more aware of what could affect their teaching practices and students' learning opportunities; hence, they were highly careful about the strategies they used. Abdelati (2019) examined the roles of CSs in secondary EFL classrooms in Libya with regard to how they help develop the communicative competence of learners. The thesis analysed the content of the teaching materials and the perceptions of both teachers and students about CSs. Findings showed that knowledge of CSs was unavailable in the teachers' professional training, which seemed to have resulted in the limited and unconscious use of CSs among the teachers observed by the researcher.

With regard to the Vietnamese EFL teaching context, research into teachers' CSs is quite scarce as CSs research has mostly focused on learners (e.g. Bui, 2012; Nguyen \& Nguyen, 2016). As for communication strategies used by teachers, some studies explored a specific strategy used by teachers when speaking English, such as code-switching (e.g. Grant \& Nguyen, 2017; Nguyen, 2013), L1 use (e.g. Bui \& Nguyen, 2014; Kieu, 2010), and the use of nonverbal communication (e.g. Nguyen, 2015). Generally, results of the mentioned studies showed that Vietnamese EFL teachers made attempts to use code-switching, L1, and nonverbal strategies to gain control of their classrooms, for pedagogical purposes, and to help themselves perform better in EFL classrooms.

Gaps for this Study. The above review indicates that empirical research into English communication in the EFL classroom settings is needed to better understand teachers' choice and the impact of CSs on students' English learning. There are four main gaps for further research into the fields of English communication in the context of Vietnamese EFL teaching. Firstly, few studies have focused on how much English and Vietnamese teachers speak in class, especially in contexts where students are non-English majors. Secondly, a more complete picture of CSs used by lecturers when speaking English in Vietnamese EFL classes has not yet been established. Thirdly, few studies have looked into how teacher language awareness is reflected in CSs use. Lastly, little research has linked teachers' practices with students' perspectives regarding the teachers' use of English in Vietnamese English teaching contexts. The current study addresses these gaps as it explores how lecturers use English communication in non-English major classes and students' perceptions of their lecturers' practices in Vietnamese EFL teaching context. 


\subsection{Classroom Language Choice}

How a second or foreign language can be taught effectively seems to be the most commonly asked question for L2/FL educators. Among the factors that decide the success of the teaching process, classroom language choice is of great importance, which is explored in greater detail in this section.

\subsubsection{L1 Use in the L2/FL Classroom}

L1 use is prevalent in language classrooms where teachers and learners share the same first language. Recent theories and research in SLA have suggested that there are two sides to the use of L1 in an L2 classroom, suggesting that the decision of language choice is not just black and white. This section examines both the negative and positive roles of the L1 in L2/FL teaching. It also presents relevant findings of empirical studies.

Quantity of L2/FL Teachers' L1 Use. Studies examining the quantity of L1 used by L2/FL teachers have shown two main trends. The first group of research findings reveal a relatively small percentage of L1 use. For example, De la Campa and Nassaji (2009) investigated how much and when teachers used L1 in two German-as-a-foreign-language classes and found an average L1 use of only 11.3\%. Song and Andrews (2009) found that the four Chinese English teachers in their study used from $10.5 \%$ to $32.2 \%$ L1 in their English teaching. Bozorgian and Fallahpour (2015) investigated the amount and purposes of L1 use in EFL classrooms in Iran and their results show that the teachers used L1 very little, from 3.14\% to the maximum of $11.33 \%$. Taşçı and Aksu Atac (2020), however, found Turkish EFL teachers used a higher amount of L1 in their classes, ranging from 21 to $30 \%$ of all classroom instructions.

The second group showed substantial variations across teachers, even within similar teaching contexts. Liu et al. (2004) examined the percentage of L1 and target language words in the lessons of thirteen high-school teachers of English in South Korea and found the teachers' L1 took up from 10 to 90 percent of the total language use. Similarly, Al-Ghafri et al. (2019) found that Omani English language teachers used their learners' L1 quite flexibly, from a minimum of $28.1 \%$ to a maximum of $71.9 \%$, in L2 classrooms. Littlewood and Yu (2011) reported that Mainland China teachers' L1 speaking time took up about 64\% in their English classes. Aly's (2020) investigation of learners' and teachers' attitudes toward using L1 in classes focused on learners of Arabic and found a rate of $67.4 \%$ L1 used by teachers. High levels of teachers' L1 
use were also noted by Lo (2015) who detected that, when teaching students with limited L2 proficiency, teachers used a dominant proportion of L1 in their lessons.

The above-mentioned research has demonstrated the varying levels of L1 use in different language learning settings. The findings suggest a preference among EFL teachers to combine L1 and L2 in the classroom. Ultimately, the quantity of L1 and L2 use is context-based and a matter that each teacher must decide for him or herself (Almoayidi, 2018; De la Campa \& Nassaji, 2009).

Arguments for the Negative Role of the L1 in L2/FL Education. In examining the role of the L1 in L2 learning, it is necessary to look back at some early theories in SLA. Behaviourists believed that "the main impediment to learning was interference from prior knowledge" (Ellis, 1994, p. 299). This can demonstrate that the bigger the differences between L1 and L2, the stronger L1's negative interference can be, as in the case of Vietnamese and English, which are distinguished from each other in basically all areas of linguistics. Similar to behaviourism, interaction hypothesis posits that using the L1 for classroom interaction can be "depriving the students of the only true experience of the L2 that they may ever encounter" (Cook, 2001, p. 409). Combining those views, it seems that L1 may cause negative interference in L2 instruction and interaction.

There have been empirical studies which evidenced that students' L1 may have negative effects on L2 acquisition. The study of Shamsudin et al. (2013) revealed that L1 interference led to collocational errors by Iranian EFL learners in both writing and speaking tests. The negative interference of L1 is supported by Jaiprasong and Pongpairoj (2020) who investigated how L1 Thai learners produced English word stress. Results illustrated that those learners made the errors because they applied the word stress assignment rules of L1 into English while the rules are different from the two languages. Savran Celik and Aydin (2018) looked over the related literature and concluded that excessive use of L1 automatically reduces learners' productivity and awareness, keeping learners' focus away from learning English. They added that L1 use prevents interaction and metalinguistic competence and thus reduces the effectiveness and richness of the language learning environment. Tsukamoto (2012) found that the use of L1 in an EFL classroom may also be demotivating for students because the students do not have many opportunities to have contact with the target language outside the classroom.

As can be seen, L1 can have a negative influence on L2/FL learning and teaching. In the Vietnamese EFL teaching context in particular, the negative interference of L1 can be caused 
by the difference between the two language systems, especially phonetic, typological, and cross-cultural differences (Giang, 2007). Two studies conducted by Pham (2015) and V. D Vu (2017) who employed error analysis of English writing by Vietnamese EFL tertiary students found evidence of L1 interference from both lexical and syntactic errors in the students' L2 writing.

Arguments for the Positive Role of the L1 in L2/FL Education. As discussed previously, L1 was regarded by the early behaviourist learning theory as having a negative effect on L2 learning due to the errors resulting from negative L1 transfer. However, behaviourists also believe that L1 transfer can also have positive effects on L2 learning. For example, Odlin (1989) examined L1 transfer in terms of semantics, phonology, writing systems, and syntax and asserted that much of L1 influence can be very helpful, especially when there are only a few differences between L1 and the target language. Sociocultural theory implies that L2 learning is viewed as a mediated process in which "L1 plays a key role in helping learners to mediate each other" (Lantolf, 2000, p. 87). Also, learners' mother language can be regarded as a crucial scaffolding support tool (Vygotsky, 1981). Villamil and De Guerrero (1996) claimed: "the L1 was an essential tool for making meaning of text, retrieving language from memory, exploring and expanding content, guiding their action through the task, and maintaining dialogue" (p. 60). Thus, certain useful functions of L1 in L2/FL classrooms have been acknowledged in L2/FL teaching and learning.

Recent research and studies have confirmed the multi-functionality of L1 use by teachers in L2/FL classrooms. Firstly, teachers commonly use L1 for pedagogical purposes such as eliciting answers, explaining meaning, answering students' questions, giving feedback, and classroom management (e.g. Ma, 2019; Mohebbi \& Alavi, 2014; Nakatsukasa \& Loewen, 2015; Nukuto, 2017). Research has suggested that the use of students' L1 as a consciousnessraising tool for the teaching of grammar is effective for beginners (Alijani \& Barjesteh, 2018; Arshad et al., 2015). Secondly, teachers utilise L1 to create and enhance interpersonal relationships and express emotions with students by making jokes, or offering students praise and encouragement (Caldwell-Harris, 2014). Teachers' L1 has been shown to be a source of humour which could minimise learners' tension and increase the learners' interactional competence in the classroom (Jawhar, 2018). Importantly, recently it has emerged that the controlled and well-organised use of L1 in EFL classrooms speeds up L2 learning process (Awada et al., 2020); boosts learners' confidence in L2 productive skills (Nilubol, 2020); and lessens the L2 anxiety level (Bukhari \& Aziz, 2020). 
Research has also found positive perceptions and attitudes of L2 students and teachers toward L1 use. Results revealed that learners' L2 competency affects their degree of agreement toward the use of L1. For example, while the higher-level students expressed a negative view of L1 use within a tertiary learning context, lower level proficiency participants had a positive attitude (Aly, 2020; Shariati, 2019). In general, researchers (e.g. Anggrahini, 2019) have reported that students supported minimal use of L1 in EFL classrooms. Teachers were found to believe that English should be the main tool of classroom communication but that the use of L1 can be useful in certain situations as long as it is kept at a minimum (Shabir, 2017; Shuchi \& Islam, 2016). In other words, it seems that a pedagogical use of L1 is favoured by both teachers and students (Sevim \& Turhanli, 2019).

Tensions and contradictions have also been found between teachers' beliefs and their practices when it comes to L1 use in the classroom. For instance, Tan (2017) found that teachers' effort to balance between judicious L1 use for learning purposes and to avoid over-reliance of L1 use is the main source of tensions for their practice. Moreover, Yuvayapan (2019) reported that, although teachers held positive views about L1 use in some particular activities, they did not frequently employ this pedagogy due to the expectations of their institutions, colleagues and the parents of their students. Nevertheless, based on a large-scale video and survey study involving 49 Norwegian high schools, Brevik and Rindal (2020) reported that most students found teachers' use of Norwegian (L1) useful as it helped them understand class content. One of the common areas where the use of $\mathrm{L} 1$ is thought to help is to provide scaffolding to guide students in their learning tasks.

\subsubsection{English-Only Instruction in L2/FL Teaching}

An English-only approach has become a popular choice in many EFL teaching contexts, and while it certainly has some proven benefits, it does not come without its challenges. It is controversial in many L2/FL teaching contexts as it reflects a monolingual approach to teaching English. English-only teaching is a central issue for both EFL learners and teachers, who have expressed a wide variety of opinions and ideas on the topic.

Benefits and Challenges of English-only Teaching. Empirical research has highlighted the practical benefits that the English-only practice brings about. Firstly, it was found that an English-only policy can make students improve their listening skills (Wei, 2013). Students who experienced English-only classes seemed to have less anxiety and feel more confident and motivated to use the target language, which is beneficial for enhancing 
proficiency (Rahmadani, 2016; Rose \& Galloway, 2019). Research also noted that English instruction programmes prepared students for the high demands of using English in real-world interactions (Shvidko, 2018); and for work life (Ekoç, 2020). English-only practice is particularly beneficial to students in EFL contexts because they are rarely able to practise L2 outside classrooms (Savaşçı, 2014).

However, an English-only approach has also been associated with certain challenges and constraints. One of these constraints pertains to students' English proficiency, which can impact learning in English-only classrooms in many ways (Beckett \& Li, 2012; Chapple, 2015). Students' heterogeneous English competency is the main challenge for teachers because it forces them to spend a lot of time dealing with finding relevant materials as well as suitable activities to match students' varying proficiency levels (Li \& Wu, 2017). Kim and Tatar (2017) also noted that it was not easy for non-native teachers to deliver lectures in English "since teachers need to address students' English proficiency in addition to other instructional concerns". Furthermore, Aguilar (2017) pointed out that low English proficiency can be a challenge for both teachers and students. In the Indonesian EFL teaching context, teachers' lack of a clear understanding of key areas such as the selection of learning materials or ways to test students' learning were found to further constrain the implementation of an English-only approach (Simbolon et al., 2020).

Therefore, for the successful implementation of English-only instruction in EFL teaching, highly qualified teachers with subject and pedagogical content knowledge, students with appropriate English-language proficiency, and well-resourced physical environment need to be considered (Selvi, 2014). Teachers are further required to develop tasks that are adjusted to the students' heterogenous skill levels, ensure students' comprehension, and create an atmosphere where students are not afraid to speak in English, while also being mindful of "the many cultural differences present in the room and the potentially different language levels of individuals" (Dearden, 2014, p. 23).

Teachers' and Students' Perceptions toward English-Only Teaching. Research has shown a complexity of attitudes toward English-only teaching by teachers and students.

Research into learners' perceptions towards English-only teaching in EFL classrooms captured both positive (e.g. Bukve, 2020; Uçar \& Soruç, 2018) and negative (e.g. Doiz et al., 2012; Lee \& Lo, 2017) attitudes. For example, Al Sharaeai (2012) studied tertiary students from different linguistic backgrounds, including Arabic, Chinese, Korean, and Spanish, and at different 
English proficiency levels and majors. Results showed that the students preferred teachers who could not speak their first language and supported English-only instruction in class, preferring to be exposed to English as much as possible. Akhter (2018) reported that undergraduate students expected their teachers to use mostly the target language in the English classrooms, explaining that the first language killed students' curiosity and discouraged them from speaking English. However, a majority of the Bangladeshi university students surveyed by Murtaza (2016) thought that the English-only practice was difficult for them. The survey data indicated that students were not comfortable with this instruction because they felt uncomfortable when having to ask and answer questions in English. Similarly, 366 Korean undergraduate students in the research of Lee and Lo (2017) did not think English-only teaching was a good practice in their classes because they still need L1 in many cases.

Teachers also seem to have mixed views on an English-only teaching approach. While agreeing with the benefits of English-only teaching, they support both L1 and L2 use in practical teaching (Simbolon et al., 2020; Thornton, 2017). For instance, Cheng's (2017) examination of EFL university lecturers' perceptions of English-only policy in Taiwan found that the lecturers had a positive view of English and they tried to use English most of the time, believing that students' listening comprehension would improve. However, the lecturers sometimes chose to switch between English and Chinese and allowed students to use Chinese to ask and answer questions to help them overcome both content and language barriers. Awaiko Westin (2019) interviewed Swedish English teachers' perceptions about English as the language of instruction. Results show that no teachers argued for the total support of 'English only'; some of them had tried to use 'English only' but regarded it as unnatural. Instead, the teachers largely concurred with the use of Swedish, or other first languages, because L1 was believed to be the best language to make comparison or give clarification.

English-only Teaching in the Vietnamese EFL Context. Like many other countries, to develop the English language proficiency of future generations of Vietnamese students, the Ministry of Education and Training recommended the use of English as the only medium of instruction at both international and domestic tertiary institutions in 2008 (Vietnamese Government, 2008). As a result, despite not being a prevalent trend, English-only teaching practices have been applied at some Vietnamese universities, particularly for teaching the English subject. However, this approach creates enormous challenges for not only Vietnamese learners but also their teachers. 
Studying how English-only instruction is effective and desirable in Vietnamese tertiary EFL teaching contexts, Nguyen et al. (2010) reported that the majority (70\%) of the students cannot fully understand lessons. Moreover, about two thirds of the students said they had difficulties using English to present their ideas with others. About 55\% of students were opposed to the English-only teaching; and around $70 \%$ of them recognised the use of Vietnamese as a beneficial teaching and learning medium. Dang and Moskovsky (2019) composed a critical review of relevant policy documents related to English-medium instruction in Vietnam's tertiary sector, including the English-only teaching in EFL classes. The findings show that the current English medium instruction policies "promote practices of direct borrowing EMI programs from oversea higher education institutions, while at the same time lacking clear guidelines or recommendations for language management, internationalisation strategies, and proper involvement of multiple agents at different levels" (p. 1343). All these abstract and decontextualised policy documents were found to result in an ineffective implementation of English-only instruction. Dang and Moskovsky (2019) recommended that Vietnamese policymakers should respond to the interests and concerns of micro-level actors (e.g. learners). The study also suggested that English-only teaching can be successful when there is effective communication between the macro and micro level.

Similar to other EFL education contexts, in the Vietnamese EFL teaching setting, the implementation of English-only teaching has been constrained by teachers' limited English proficiency, students' low English competence and passive study habits, ineffective teaching methods, and inadequate classroom resources (Nguyen et al., 2016; Vu \& Burns, 2014). Those have partially accounted for the more popular and favourable choice of the bilingual approach in EFL contexts in Vietnam.

\subsection{Teacher Language Awareness and Perceptions}

In addition to teaching methods and techniques, teacher-related factors also have an influence on classroom teaching and students' learning. In FL/L2 education settings, teachers' knowledge and understanding of the structure of English contribute to their effectiveness as teachers (Andrews, 2007). This section discusses teacher language awareness and perceptions of English language teaching.

\subsubsection{Teacher Language Awareness}

Definitions and Concepts of Teacher Language Awareness. Teacher language awareness refers to 'teachers' cognitions (knowledge and beliefs) about language in general 
and the language they teach" (Andrews \& Svalberg, 2017, p. 219). For language teachers, language awareness involves both language proficiency and metalinguistic knowledge of the language being taught (Andrews, 2001). Language proficiency is the skill level that an individual can speak, write, read, or understand a language (Tavakoli et al., 2014). Teacher language proficiency is central to language learner success because the likelihood of a learner succeeding is minimal when a teacher is not proficient (Dippenaar \& Peyper, 2011). Metalinguistic knowledge refers to having knowledge of the general rules and universal templates of language structures (e.g. nouns, verbs, adjectives, adverbs, word order, morphological patterns) (Bialystok, 2001). Language teachers are required to have "high levels of language proficiency to be able to present rich and well-formed patterns for their students as well as an adequate explicit knowledge about language to respond properly to learner needs" (Hayati et al., 2017, p. 87). Explicit language awareness and metalinguistic knowledge "are particularly important for language teachers, who may be required to provide explanations of grammatical forms or usage norms to their students." (Riordan, 2018, p. 148). In addition, for EFL teachers, general and classroom English proficiency are useful in building the teachers' confidence and raise their awareness; classroom English proficiency can bring the teachers a sense of comfort in practical use of English to teach English, helping them to choose suitable expressions and better manage the class (Pham, 2018). However, language proficiency and metalinguistic knowledge of the language alone seem to be insufficient in EFL teaching as teaching English through English "requires consideration of a number of related issues, since in language teaching, language is both the content of teaching as well as the means by which it is taught" (Parab, 2020, p. 52).

Three Domains of Teacher Language Awareness. Andrews (1997) argued that L2 teachers should be aware of their learners' language skills, including learners' perspectives, awareness of interlanguage development and the degree to which the language content in the materials and lessons poses difficulties for the learners. In this regard, a linguistically aware teacher not only masters how language works but also understands how their students "struggle with language and [is] sensitive to errors and other interlanguage features" (Wright, 2002, p. 115). To do this, teachers should sometimes take the role of learners to be aware of their difficulties in comprehension and output performances. Also, L2 teachers' interactional awareness is an essential element of teacher language awareness because teachers' knowledge of interactional processes can "create the real, inside world of the L2 classroom" and thus "result in a wider range of opportunities for learning" (Walsh, 2003, p. 125). Teacher language 
awareness also includes an awareness of "the challenges posed for the learners by the language content of pedagogic materials and tasks" (Andrews, 2007, p. 175).

As can be seen, teacher language awareness requires L2 teachers to take on different roles: experienced language teachers with deep knowledge about learners, effective language users, language analysts, and effective mediators/facilitators. Taken all together, Andrews (2007) viewed teacher language awareness as the combination of three domains:

(1) User domain: Teacher takes the role of a language user; the language proficiency ensures him/her to function as a model and to provide diversified and well-formed input for learners; communicative competence is crucial (Celce-Murcia, 2008)

(2) Analyst domain: Teacher takes the role of a language analyst; his/her metalinguistic knowledge of the language systems and ability to understand how the target language works enables him/her to offer explanations of grammatical structures and to respond to learner errors; metalinguistic awareness is important (Van Lier, 1998)

(3) Teacher domain: Teacher acts as a language teacher; his/her theoretical knowledge about the pedagogy and principles of language teaching helps him/her to conduct the teaching procedures and plan instruction that engages and supports a wide range of language learners.

Andrews' framework has recognised and synthesised three most salient domains of language teacher awareness: user, analyst, and teacher. Therefore, it is found to be appropriate for this study when investigating how teacher awareness affects EFL teachers' use of communication strategies.

Additionally, language teachers need to adjust language input to make it more accessible and comprehensible to learners (Elder, 2001). According to Andrews (2001, 2007), teacher language awareness has a great deal of influence on the quality of input they make available to the students. This input is "language contained in materials, language produced by other learners, and language produced by the teacher" (Andrews, 2007, p. 39). In other words, a language teacher's metalinguistic awareness interacts with the language produced by the three main sources of input for learners in a L2 classroom: teaching materials, other learners, and the teacher.

When referring to L2 teacher cognition and teaching knowledge, Freeman (2016) identified content knowledge, pedagogical knowledge, and discourse skills as three interrelated aspects. Content knowledge refers to teachers" understanding about "their own subject area determining teacher effectiveness" (Buendía-Arias et al., 2020, p. 587). Pedagogical knowledge is linked to 
teachers' own learning experience and practical knowledge developed during their teaching career (Akbari \& Dadvand, 2014). Especially, pedagogical learner knowledge which refers to as 'teachers' knowledge of the learners in all their richness and complexity with which they develop their personal and interpersonal skills as well as sensitivity and ability to accommodate their subject-matter knowledge and knowledge of L2 teaching to their learners' varied learning trajectories" should be taken into special account (Le, 2020, p. 77). This knowledge enables L2 teachers to decide the optimal conditions for language use needed by particular groups of learners and to activate the learners' mental processing capacities for language to emerge (Le, 2020). Discourse skills demonstrate critical and adequate knowledge about learners and the environment, comprising the ability to maintain comprehensible, fluent, and accurate communication (Parab, 2020). These aspects of teachers' knowledge can provide "a useful analytic framework to help understand the nature of teaching English through English" (p. 52).

Teacher Language Awareness and Pedagogical Practices. Teacher language awareness, especially in L2/FL classrooms, has been shown to have a significant impact on pedagogical practices. Andrews (2001) believed that teachers' language awareness has a profound impact on the teachers' performance of many tasks in the classroom such as making input comprehensible to learners, making key grammatical features salient, or providing clarification and exemplification. Degrees of teachers' language awareness also enable them to perform such pedagogical tasks such as responding to language-related questions raised by learners and recognising dialectical varieties among learners (Andrews \& Lin, 2017). The level of teacher language awareness largely determines students' engagement in classroom practices (Andrews, 2007).

Teacher language awareness also plays a pivotal role in their use of communication strategies, regardless of which definition of communication strategies one adheres to (see 2.2). For instance, from a traditional view, Faerch and Kasper (1983) claimed that awareness results from difficulties or deficiencies that require target language users to resort to a compensatory strategy. Those users intentionally employ one of the strategies to deal with such difficult situations as facing limitations of lexical resources. From an interactional perspective, awareness belongs to a property of discourse where both speaker and interlocutor realise it as a resource to counter deficiency and to negotiate input (Yule \& Tarone, 1991). As can be seen, despite differences in the origins and purposes of communication strategies, both the traditional and interactional approaches confirm speakers' consciousness in using those strategies. 
From the discussion above, a close connection between teachers' language awareness and their approach to communication in the classroom has been identified. Since a teacher's English communication is an important source of input for learners, his/her language awareness has a profound impact on this also. Because communication strategies are an underlying mental human process (Bialystok, 1990), teachers need to know about and be able to intentionally use a range of these strategies. Teacher language awareness is argued to enable teachers to provide strategies such as exemplification and clarification to make the target language input more comprehensible to students. It can, therefore, be assumed that teacher language awareness is a key factor accounting for teachers' practices and perceptions about language use and communication strategies when speaking English in the classroom.

\subsubsection{Teacher Perceptions}

The term 'perception' is generally understood as "the way an individual observes, understands, interprets, and evaluates a referent object, action, experience, individual, policy, or outcome" (Bennett, 2016, p. 585). In educational contexts, teacher perceptions are defined as:

the ability of teachers to recognize or become aware of something when they use their senses such as seeing or hearing (Alsolmi, 2017).

the feelings teachers have while teaching; and if teachers' perceptions are positive, teaching will produce better results than if the perceptions are negative (Roman Etxebarrieta et al., 2020).

Despite differences in expression, these definitions have indicated that teacher perceptions are a personal and cognitive process and thought to have a strong influence on their teaching practices and students' learning.

Sources of Teacher Perceptions. Teacher perceptions have been found to be influenced or shaped by "a complex and extended process of socialisation" (Meier, 2010, p. 173). Firstly, social forces can tremendously affect how teachers perceive their teaching practices and students' learning (Fang, 1996; Salama, 2018). Social changes can result in teachers having biased perceptions about some of the things they deal with on a daily basis (Andrew, 2013). Secondly, teacher perceptions are shaped by their teaching experiences, particularly by how they experience students and teaching subjects (Levin \& Wadmany, 2008; O'Bannon \& Thomas, 2014; Tobin et al., 2009). Thirdly, teacher knowledge gained through prior language learning experiences and professional teacher education also has a significant influence on their perceptions (Blume et al., 2019; Borg, 2003; Busch, 2010). 
When it comes to L2/FL teachers, their knowledge base is found to deeply shape the teachers' perspectives of their language teaching and students' learning. For instance, teacher perceptions are shaped by content knowledge, pedagogical content knowledge, knowledge of the curricula and general educational knowledge (Silva et al., 2019). Teachers' previous L2 learning experiences and knowledge they received may have a significant impact on the ways they teach, which have an impact on learners' attitudes and, thereby, their learning achievements (Baker, 2011; Park \& Sung, 2013). Professional education and training that L2/FL teachers received may also affect and shape the teachers' perception of L2 teaching (Durán-Narváez et al., 2017; Farrell \& Guz, 2019; Moodie, 2016).

\subsubsection{How Language Awareness and Perceptions Influence Teachers' Communication Strategies in Classrooms}

As mentioned earlier, English teacher language awareness is constituted by three domains. As language users, teachers function as English-speaking models who aim to provide well-formed input for learners. This requires their English performance to be natural and fluent, which can create tensions for them because students' proficiency may not good enough to follow fluent English. As analysts of language, teachers understand syntax and grammatical knowledge of English. Hence, they tend to correct their own mistakes and give corrective feedback to students, which may affect the teachers' and students' English-speaking fluency. As language teachers, they must master English teaching pedagogies in order to conduct the most effective lessons and meet their students' demands. This requires them to be mindful of the amount of English use, levels of difficulty, and techniques that should be applied to make their English comprehensible and accessible to learners. Also, as has been suggested above, the enactment of teachers' perceptions is affected by different factors, which can sometimes make teachers unable to translate their perceptions into practices. This means that teachers' perceptions may be different from and even conflict with their actual practices of using classroom English communication.

\subsection{Affective Factors in Second Language Teaching and Learning}

The strategic role of the affective domain in SLA has been established for decades. The affective domain refers to "the emotional side of human behaviour" and it may be "contrasted to the cognitive side" (Brown, 2014, p. 142). Krashen (1988) posits that affective factors function as a filter that helps or hinders learners' reception of language input and impacts their comprehension level. According to Ni (2012), affective variables determine the amount of 
language learners' input and intake; thus, affecting the acquisition of that language. In order to examine teachers' classroom English communication, four important affective factors need to be taken into account. They are sense of belonging, expectations, relationships, and motivation, which are in turn presented in this section.

\subsubsection{Sense of Belonging}

Sense of belonging is described as a person's "sense of identification or positioning in relation to the group or to the college community, which may yield an affective response" (Tovar \& Simon, 2010, p. 200). The sense of belonging and the need to belong are recognised as so essential that they are referred to as a fundamental human need (Baumeister \& Leary, 1995). When a person's need to belong is satisfied, they will have positive emotional reactions, try to maintain interpersonal relationships, and design goal-directed activities to keep the need to belong continual (Song et al., 2020).

In the higher education context, sense of belonging reflects the sense that "one fits, matters, or belongs on the college campus and in the college community" and "influences student behaviours that lead to a higher probability of returning to college and to higher affective outcomes" (Lancaster, 2020, p. 3). Sense of belonging refers to students' perceived social support, feeling of connectedness, and experience of being cared about, respected, and important to the school or class (Strayhorn, 2018). These definitions indicate that students' sense of belonging consists of both cognitive and affective factors: cognitive assessments lead to students' affective responses.

School belonging plays an important role in students' school life (Korpershoek et al., 2019). Research (e.g. Cemalcilar, 2009; Henry, 2020; Thomas, 2012) has shown a strong positive relationship between students' sense of belonging in schools and the achievement of positive educational outcomes. A strong sense of belonging is positively associated with students' social-emotional functioning in school (Korpershoek et al., 2019), as well as the development of emotional engagement (Buhs et al., 2006). Students' feelings of belonging can enhance their academic competence and expectations (Hernández et al., 2016). Therefore, it is an important outcome for universities to generate in students a strong sense of belonging to improve their experiences and attainment, as well as to reduce attrition rates and ensure student success (Peacock et al., 2020; Thomas, 2012).

In EFL classes, teachers' encouraging words and thoughtful suggestions are important for building a sense of belonging (H. P. Q. Pham, 2017). EFL teachers need to create a supportive 
classroom atmosphere and use interesting activities or visual means (e.g. pictures which contain English proverbs) because the classroom setup can serve to create a sense of belonging to the class itself (Alhodiry, 2016). When EFL students perceive the class and group which they belong to as welcoming and supportive, they are more likely to persist and seek help when needed (Cuellar \& Johnson-Ahorlu, 2016; Museus et al., 2017). Thus, creating an emotionally supportive learning space is essential in developing a positive sense of belonging to the class among EFL students.

\subsubsection{Expectations}

The learning environment and emotional states of those involved in the classroom setting can be strongly affected by students' and teachers' expectations (Sánchez et al., 2013). Expectation is defined as the act or state of looking forward or anticipating (Fauzi et al., 2019), and "subjective judgments about how likely it will be that certain future events will occur or not occur" (Treiber, 2019, p. 18). Research into SLA has pointed to teacher and learner expectations as one of the variables that can influence the L2/FL teaching and learning process (Nhapulo, 2013).

Sociocultural theory conceptualises language teaching as being shaped by the teaching environment, which means that the reality of teaching should not be dealt with separately from its cultural and social context. Thus, it is likely that L2/FL teachers' expectations are diverse and can vary from culture to culture. For instance, Chinese teachers have been found to have high academic expectations for all the students due to the belief that everyone can make progress in their academic study as long as they are willing to work hard (Zhou \& Li, 2015). These expectations not only affect their teaching behaviours and practices but also influence their relationships with students (Kazemi \& Soleimani, 2016). Research also suggests, however, that when L2/FL teachers' expectations are too high and unrealistic with regard to students' academic success, their overall achievement can be negatively affected (Tsiplakides $\&$ Areti, 2010). Therefore, L2/FL teachers need to have "a high but realistic expectation for students' achievement” (Mulisa \& Kassahun, 2019, p. 30).

Like teachers, L2/FL students also bring with them a set of expectations about teaching, learning, and assessment. Recent research (e.g. Ellen \& Taverniers, 2011; Tergujeff, 2013) has highlighted that many students expected their teacher to have native speaker-like pronunciation. Lobo and Gurney (2014) found that students expected to be taught certain aspects of the target language such as useful English academic and professional phrases and 
vocabulary that they would be able to employ in future jobs. Obeidat (2020) surveyed Jordan EFL students' expectations, reporting that students were more interested in improving their communication skills rather than learning about grammar and sentence structure. In Vietnam, Hoang (2017) explored both teachers' and students' expectations in the Mekong Delta of Vietnam as an EFL setting. Results suggested a gap between the goals set by the Ministry of Education and Training and the expectations of both teachers and students. Specifically, students anticipated that the English course book would include more information relating to Vietnamese life, people, and culture, as well as the topics that are of their interest.

Both teachers and students come to the language classroom with expectations about what teaching and learning should look like, and if their expectations are not fulfilled, negative emotions can arise (Cuéllar \& Oxford, 2018). Mismatches between students' expectations and reality "can result in lowered motivation, disengagement and attrition" and can "severely impact upon student behaviour in class and their overall engagement with the course" (Lobo \& Gurney, 2014, p. 731). Therefore, teachers would benefit from understanding students' expectations in EFL classrooms. At the same time, students should also be aware of their teachers' expectations of them and the reasons for those expectations so that the teachers' and students' expectations, behaviours, and performances can align, resulting in a more positive learning environment.

\subsubsection{Relationships}

The teacher-student relationship is defined as "an interpersonal relationship between a teacher and a student that contains whatever level of respect, understanding, and care that both require to make the relationship work for them" (Gibbons, 2019, p. 12). From this definition, respect, understanding, and care have been identified as the three key elements that can foster a productive teacher-student relationship. If a teacher behaves in a respectful and caring manner towards students, they may feel more confident and comfortable to approach the teacher and share personal and academic issues with them; thus, a successful teacher-student relationship can be established (Sánchez et al., 2013). Students develop positive relationships with their teacher when both parties treat each other with respect (Krane et al., 2016) and when the teacher understands the students' psychological needs, such as the need for autonomy, competence, or learning preferences (Smit et al., 2014). Teachers' demonstrations of care for their students were found to give students the feeling that the relationship between them and the teacher is strengthened and that their learning needs can be met (Calhoun, 2019). Because every individual in the classroom participates in a relational process in which expectations are formed 
and information is shared, the relationships that evolve between a teacher and learners influence learning directly and indirectly (Frymier \& Houser, 2000). This effect, however, is two-sided: a positive relationship helps students excel in terms of their academic achievements while a negative relationship can lead to significant problems that can affect students' learning (Agyekum, 2019).

A positive and effective relationship between teacher and students has been shown to bring about many benefits in L2/FL educational contexts. Research findings have indicated that good teacher-student relationships benefit performance-related variables in L2/FL classes. For example, they can lower students' anxiety and allow the students to feel more comfortable to speak in English (Nijat et al., 2019; Wei et al., 2009). Teachers' respect for students strongly affect their learning motivation and willingness to communicate in the target language (Joe et al., 2017). An effective relationship can foster students' English self-efficacy and the use of learning strategies and thus help the students develop English proficiency (Ma et al., 2018). In fact, a good rapport between students and teachers can raise students' attitudes towards and interest in learning English in the classroom (Bouras \& Keskes, 2014). Hence, according to Girón Chávez et al. (2017), EFL teachers need to create a respectful and cooperative atmosphere so that students feel confident enough to communicate their needs and learn effectively. This is of greater importance for students who have low motivation and lack interest in learning English, as may be the case for non-English major students.

On the other hand, a negative and ineffective teacher-student relationship can act as an obstacle and hinder the teaching and learning process (Davis, 2001). A negative relationship between teacher and student can make students skip school, not work hard, face poorer academic outcomes, and display a large number of behavioural problems (Harrison et al., 2007). Sharing negative relationships with teachers can also hamper students' engagement in class (Roorda et al., 2011). It was also shown that a negative relationship decreases closeness and increases conflict (Armstrong et al., 2017; Pianta et al., 1995).

In addition to respect, understanding, and care, research has also identified other factors that can affect the relationship between teacher and students. When lecturers and students have different ideas of what constitutes a learning opportunity, perceptual mismatches between them occur (Kumaravadivelu, 2003). Those mismatches are prevalent in education (Khodamoradi et al., 2019). Thus, teaching must start with solving lecturer-student contradiction by reconciling the contradictory poles so that both of them are simultaneously lecturers and students (Freire, 1970). Absence of interpersonal communication negatively affects student-teacher 
relationships, which causes students to conceal their ideas in the lessons and to underestimate the discipline the language learning environment requires (Uysal \& Güven, 2018). In most Asian, typically Vietnamese education contexts, students are stressed with too many academic learning programs and teachers face work overload. Hence, both teachers and students can feel they are placed in difficult situations, which might create conflicts in their relationships (Huynh, 2017).

It is essential to build a good rapport and simultaneously avoid a poor relationship between teacher and students in order to enhance the efficiency of teaching and learning in all educational settings, including EFL teaching contexts. Teachers can use open in-class discussion as a way to demonstrate their commitment to collaborative learning and bridge the barrier between teachers and students (Brookfield \& Preskill, 2012). However, in Vietnamese culture, the hierarchical order is clearly reflected in unequal relationships such as of parentchild, man-woman, or teacher-learner (T. N. A. Pham, 2017). Of those dyads, the teacherlearner relationship is considered to be particularly hierarchical and formal (Signorini et al., 2009). In the classroom, "students are expected to be recipients of knowledge and strictly follow what the teacher expects them to do ... and teachers' roles are assumed as the source of knowledge, the knowledge transmitter, and the moral guide" (Nguyen, 2019, p. 24). Nevertheless, current international learning theories and pedagogies have changed traditional cultural practices. Like other Asian countries, English teaching and learning in Vietnam has gradually moved from the teacher-centred toward learner-centred approach, and from grammar translation to communicative language methods. The changes in teaching approaches have contributed to a closer teacher-student relationship.

\subsubsection{Motivation}

Motivation is one of the determinants of L2/FL learning achievement (Dörnyei, 1994). It is an "important affective variable in second language acquisition" and closely linked to "language achievement and proficiency" (Wang, 2006, p. 32). In the case of teachers, motivation is an important predictor of high-quality teaching (Fong et al., 2019). Motivation of L2 teachers "is complex in that it fluctuates while interacting with sociocultural factors in the dynamic context of the teaching profession" (Song \& Kim, 2016, p. 136).

It is important to discuss ways to increase students' L2/FL learning motivation level. Dörnyei (2001) proposed a motivational strategy framework for L2 teachers to enhance students' motivation to learn an L2. There are four main dimensions in his motivational model: a) 
creating the basic motivational conditions, b) generating initial motivation, c) maintaining and protecting motivation, and d) encouraging positive self-evaluation (p. 29). Basic motivational conditions can be created via a pleasant and supportive atmosphere such as comfortable physical setup of classrooms. Teachers should generate students' initial motivation by increasing the learner's "language-related values and attitudes" (p. 51) or "making the teaching materials relevant for the learners" (p. 29). It is important for teachers to maintain learners' motivation by promoting their autonomy and creating interesting lessons. Lastly, positive selfevaluation among students can be encouraged by providing motivational feedback and increasing learner satisfaction.

In addition to Dörnyei's (2001) motivational strategies model, researchers have found other ways that can help teachers motivate students in L2/FL/English learning. Teachers' use of humour can improve students' motivation to learn English; it makes them more cheerful because of the shared laughter and an intimate personal rapport between students and teachers (Hidayanti, 2019). Students who have established positive connections with their teachers may be more willing to listen to L2 and have better L2 learning motivation (Ballester, 2015; Habash, 2010; Sheybani, 2019). An example for this is provided by Kurt and Kurt's study (2018) at Namik Kemal University, which found that an instructor's enthusiasm and frequent communication at an interpersonal level with students were crucial to motivate students in learning English. A comfortable and relaxed classroom atmosphere is linked to student learning persistence and can create good motivation in EFL learning (Mutlu \& Y1ldırım, 2019). As for non-English major students, the main type of motivation demonstrated by them is professional development motivation (Lobo \& Gurney, 2014); thus, content of speech and teaching related to students' future job can motivate them.

When talking about learner motivation, teacher motivation cannot be ignored as it makes an important contribution to their professional practices, pedagogical achievements (Agezo, 2010; Salifu \& Agbenyega, 2013).

Researchers have discussed levels of motivation and demotivation of EFL teachers (e.g. Hülya \& Kanatlar, 2018; Tziava, 2003) as well as the factors that cause them (e.g. Gadella Kamstra, 2020; Suwaed, 2018). Student-related factors were found to have the highest influence on teachers' motivation (Pourtoussi et al., 2018). Those factors included low/lack of learning motivation and interest, and disruptive behaviours of students (Aydin, 2012; Javadi \& Mohammad, 2014). Students' behaviours, enthusiasm for learning, motivation, attitudes and actions can directly influence the motivation of teachers (Pourtoussi et al., 2018; Sugino, 2010). 
Mercer et al. (2016) stated that teachers' teaching can be affected by learner psychology and pointed out that the teaching environment and student motivation could influence teachers' emotions.

To sum up, there is a mutual reinforcement between teacher and student motivation (Bernaus \& Gardner, 2008; Dewaele \& Mercer, 2018). Motivated teachers can contribute to their student's success in learning English because they are more likely to produce challenging, effective, and creative lessons (Bajorek et al., 2014; Bernaus et al., 2009). Conversely, motivated students may also have a positive impact on teacher motivation and their teaching practices (Gadella Kamstra, 2020). 


\section{Chapter 3: Methodology and Methods of the Research}

This chapter firstly provides an account of the methodology employed in this investigation. This comprises the research paradigm with its philosophical underpinnings, the chosen approach, and the design of this mixed methods research. The research context, the recruitment and selection of participants, and ethical considerations followed in conducting the research are then explained. Additionally, data collection instruments, including classroom observation, questionnaires, and interviews, and the reasons behind their selection for this study, are discussed. This is followed by an overview of the data collection procedures and the presentation of the pilot study. Next, the process of data analysis is described, followed by an outline of the issues of data validity and trustworthiness of this research. Finally, the challenges and possible limitations related to the methods and circumstances surrounding the data collection are highlighted.

\subsection{Research Paradigm}

A research paradigm is referred to as a "set of beliefs, values, and assumptions that a community of researchers has in common regarding the nature and conduct of research" (Johnson \& Onwuegbuzie, 2004, p. 24). It guides researchers' choice of research methodology, which then determines their choice of appropriate methods and procedures (Creswell, 2009). An awareness of a research paradigm can "influence mixed methods design, theory use, relationships, inferences, and data reporting” (Shannon-Baker, 2016, p. 331).

In this study, I adopted a pragmatic worldview which allows me to choose any methods, techniques, and procedures of research that meet the research purposes (Creswell, 2013, Morgan, 2007). Pragmatism considers the research problem to be central and that all relevant approaches that are needed to understand that problem can be applied (Tashakkori \& Teddlie, 2016). This research involves both observations, which serve to find out the actual language use in the classroom, and interviews, which aim at understanding the perceptions of respondents. In this sense, the proposed design fulfils the guidelines of pragmatism to employ appropriate methods to explore the phenomenon under investigation (Clark \& Ivankova, 2015). Since pragmatism pays careful attention to "the value of experiences, and practical consequences, actions, and understanding of real-world phenomena" (Creswell, 2011, p. 276), it allowed me to construct rich understandings of the life experiences of teachers and students in real-life settings. Also, pragmatists aim at "more than creating knowledge based on current situations [] but making controlled changes to the reality of focus [] and on the future state of 
things" (Torrech, 2018, p. 69). Hence, this paradigm enabled me to make recommendations for possible future changes based on the current findings. Lastly, the pragmatic paradigm allowed me to emphasise the what and how of the research problem by using both quantitative and qualitative data (Creswell, 2013).

I chose a mixed methods research methodology as a mode of inquiry. A mixed methods approach "involves the collection, integration, and interpretation of both qualitative and quantitative data" (Creswell, 2015, p. 2). There are three main reasons for my choice of a mixed methods research. Firstly, as shown in the above definition, "the use of quantitative and qualitative approaches in combination provides a better understanding of research problems than either approach alone" (Creswell \& Plano-Clark, 2007, p. 5). This study aimed to investigate classroom communication discourse with a focus on lecturer talk in the natural context of the classroom. The study also sought to shed light on the role that the first language plays in creating learning opportunities in EFL classrooms in Vietnamese tertiary classrooms. Hence, there is a need for descriptive data collected from observing and interacting with the participants in the form of words or pictures rather than numbers (Bogdan, 2007). This investigation also sought a better understanding of the connection between students' experiences with learning English and their perceptions of their lecturers' use of language in the classroom. This required the collection of a larger database, which required the application of the quantitative method. While qualitative data is best suited for 'how' and 'what' questions, quantitative data typically answers the 'why' questions (Creswell, 2015). Therefore, I found that integrating the two methods could help me answer different types of questions to achieve the research aims. Secondly, combining quantitative and qualitative research is a form of triangulation and therefore can help develop better-validated conclusions by comparing numerical results obtained from a quantitative survey with thematic results obtained from qualitative interviews (Bryman, 2006; Clark \& Ivankova, 2015). Thirdly, another rationale for adopting a mixed methods approach came from the concept of complementarity. Different methods can clarify and supplement each other to address the complexity of a research phenomenon, generating a greater diversity of views and understandings (Bryman, 2006; Tashakkori \& Teddlie, 2016). Consequently, this mixed methods approach is suitable for the purposes of my research, which aims at a deep understanding of behaviours and beliefs which are usually complicated and unpredictable.

Based on the above discussion, the adoption of the pragmatic research paradigm and the mixed methods approach contributed to this current research in the following ways: 
- It provided the opportunity to approach and study the participants (lecturers and students) while they are in their natural settings (EFL classrooms).

- It offered the flexibility to employ different data collection methods to gain a deeper understanding of participants' beliefs that underpin their behaviours.

- It enabled me to create practical solutions to the research problems.

\subsection{Research Design}

The description of the study's research design presented in this section focuses on the following three aspects: (1) my epistemological stance; (2) the nature of the research problem; and (3) the data collection methods (Creswell, 2013). This study explores EFL classroom discourse in two tertiary settings to identify and learn more about the communication strategies and language use in these real classroom practices. It also provided a space to explore the perceptions of lecturers towards their use of English communication as well as students' perspectives of barriers and affordances to their learning. Specifically, this study adopted a convergent parallel mixed methods design so as to have a more complete understanding of the research problem by obtaining different but complementary data which enhance the study's validity (Creswell \& Plano-Clark, 2011). The research design is shown in the diagram presented in Figure 3.1.

\section{Figure 3.1}

The Convergent Parallel Mixed Methods Research Design (Adapted from Creswell and PlanoClark, 2011)

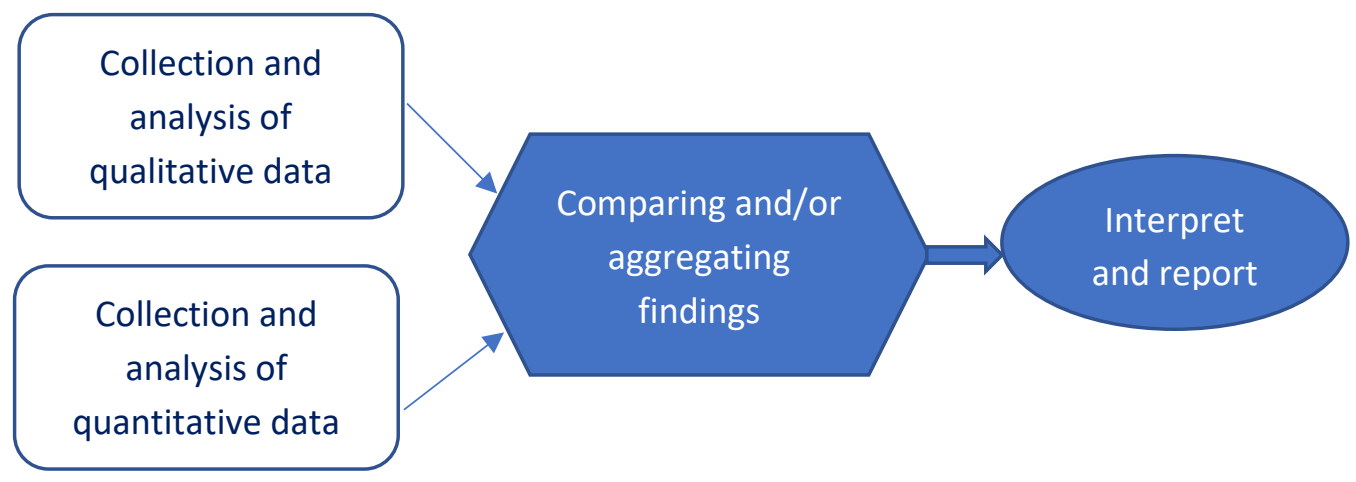

Case studies are suitable for the exploration of a phenomenon based on perceptions of a group of people who establish a case of interest within a bounded system in its natural setting (Yin, 2014). This approach is the best choice for researchers wanting "to investigate an issue in depth and [to] provide an explanation that can cope with the complexity and subtlety of real-life 
situations" (Denscombe, 2014, p. 55). Case study research "provides a way of investigating connections, patterns and context, and of reflecting on the bigger picture as well as on the detail" (Atkins \& Wallace, 2012, p. 3). In education research, using a case study approach both generates knowledge and enhances overall teaching quality (Mills et al., 2010). The major objective of case study research is any combination of "description, understanding, prediction, and control" among which "deep understanding of the actors, interactions, sentiments, and behaviours occurring for a specific process through time should be seen as the principal objective" (Woodside, 2010, p. 6). The above-mentioned benefits, principles, and objectives of a case study approach in education research were found to be relevant to the characteristics and philosophical views of the pragmatic paradigm chosen for this study. Hence, in an attempt to explore lecturers' real practices and have an in-depth understanding of participants' perceptions, I believe that a case study approach is an appropriate method.

However, "how case study researchers should contribute to reader experience depends on their notions of knowledge and reality" (Stake, 1995, p. 100). Case studies emphasise "experiential knowledge of the case and [pay] close attention to the influence of its social, political, and other contexts" (Stake, 2005, p. 444). Thus, optimising readers' experiences with the case requires researchers' meticulous selection of key issues, various procedures of meaning clarification, and a deep understanding of the case, including its social milieu and backgrounds. Taking Stake's suggestions, I applied prolonged field engagement in order to have experiential knowledge about the case, which in this case is defined by the particular EFL classroom setting. I also used data triangulation (see 3.10) to identify the different realities of the participants and enhance readers' understanding of the case.

An embedded single-case study was chosen for this thesis for two main reasons. Firstly, a single-case approach focuses on one set of contextual conditions, thus, it enables me to explore the existence of relevant phenomena more effectively, compared to a multiple-case stance (Siggelkow, 2007). In this study, I focused on the context of EFL teaching for non-English major students at tertiary institutions in Vietnam. This single-case approach allowed me to collect in-depth data related to this particular context. In other words, a single case approach enabled me to develop a deeper understanding and to provide a rich description of the studied phenomenon (Gustafsson, 2017). Secondly, an embedded design allowed me to have two levels of data analysis: each unit separately or all of the units combined (Yin, 2014). Thus, this design allowed me to access rich analysis, which can help detect a more salient picture of the main case. 
This thesis investigates five lecturers from two colleges which shared a lot of similarities in terms of the backgrounds of students and lecturers. Hence, they were treated as a group to form a single case for data analysis and each lecturer together with his/her students formed a unit of data analysis, as illustrated in the Figure 3.2 below.

\section{Figure 3.2}

The Embedded Single-Case Study Design (Adapted from Yin, 2014)

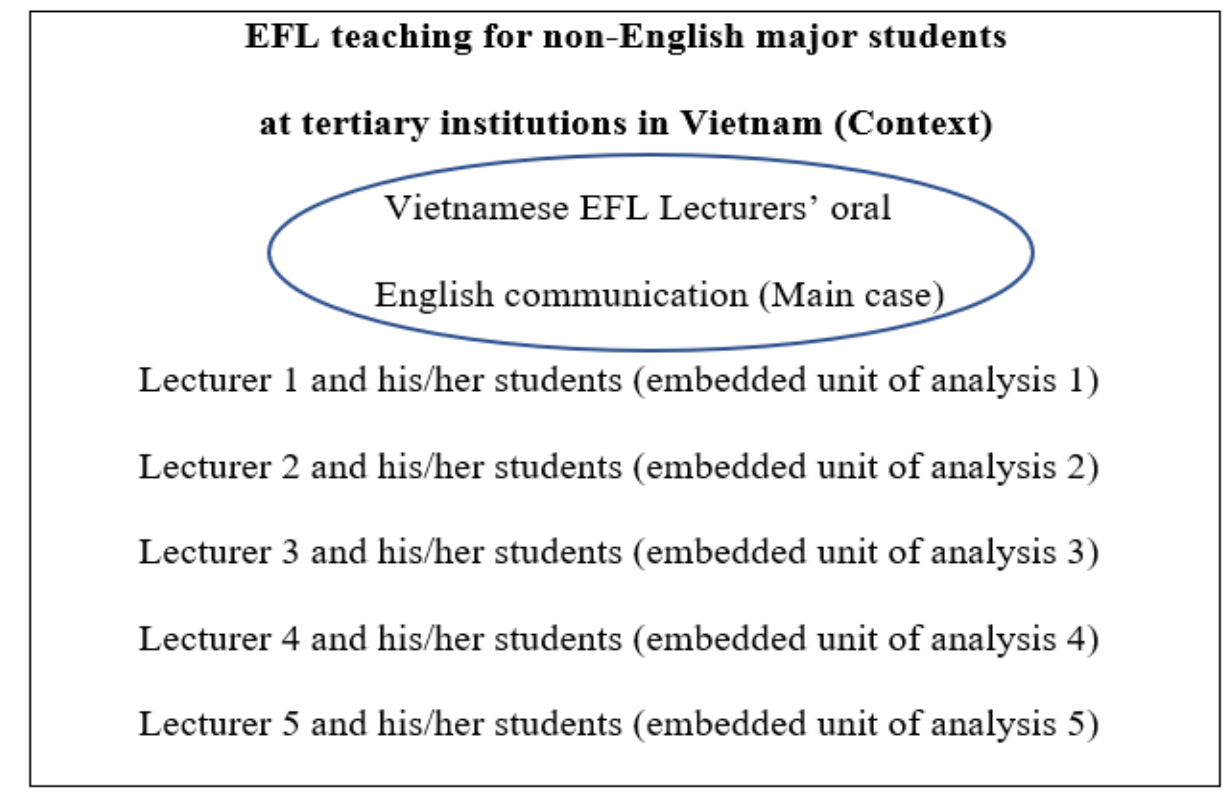

The next section describes the research contexts and the participants' background as well as how the participants were recruited and selected for data collection.

\subsection{Research Setting}

\subsubsection{The National Foreign Languages Project 2020}

The National Foreign Languages Project 2020 with its original phase 2008-2020 has contributed to setting the threshold of language proficiency levels for both language teachers and learners at all educational levels in Vietnam. Pedagogically, the National Foreign Languages Project encourages student-centred approaches to be widely applied in the English curriculum, aiming at enhancing students' creativity and engagement in the learning process. The Project has also facilitated innovative curriculum development and English teacher education by sending teachers abroad to increase their English proficiency and research competency (Bui \& Nguyen, 2016). The official letter 2196/BGDDT-GDDH was promulgated by the Ministry of Education and Training regarding the training output standards for tertiary students. Accordingly, all tertiary graduates have to achieve B1 level according to the Common 
European Framework of Reference for Languages (CEFR) - a Vietnamese version in English proficiency. In spite of all hopes and efforts, the Project 2020 was reported to be impracticable in terms of overspread and unrealistic goals, as less than $50 \%$ of the set tasks were fulfilled after two thirds of the designated timeframe (2008-2016) (Bui et al., 2018). Up to the end of 2019, very few universities and colleges had applied the English output standard for their graduate students, even for English-major students. This was because the rate of students achieving a standard level of English equivalent B1-CEFR (Vietnamese version) was low, about one third, meaning that applying the standard would result in a majority of students being unable to graduate.

Thus, a new phase 2017-2025 of the 2020 Project was launched by the Vietnamese government in late December 2017. Similar to the first phase (2008-2016), the three main goals of the new phase are renovating foreign languages education at all levels, improving learners' foreign languages competence, and enhancing the quality of the nation's human resources throughout the integration period (Vietnamese Government, 2017). Up to the middle of 2020, a number of tertiary institutions have officially stipulated and radically applied the output standard of English for their students, both non-English and English major students. Some big universities, such as the National university of Ho Chi Minh, even stipulated a standard output IELTS score of 7.0 for their English majors (NLĐ, 2020).

\subsubsection{The Two Research Sites}

This study was conducted in two public tertiary colleges in Da Nang city (Vietnam) where students undertake 2.5-3.5-year college degrees and where English is taught as a non-major subject. Students from college A were enrolled in two main training courses. The first group were trained to become programmers, graphic/web designers, and architects. The other group were taught to work in the commerce sectors. The students in college B were trained to work in tourism or catering-service enterprises, which normally have high requirements of English skills, especially communicative English. Therefore, compared to those at school A, those students should be more aware of the important role of English for their future career. This awareness may affect the way lecturers at this school teach English as well as students' motivation to learn this subject because there appears to be a close connection between career motivation and engagement in learning (Shin et al., 2016).

The English teaching pedagogies for all classes aimed at developing students' necessary output English language skills equal to the B1-CEFR level - a Vietnamese version. The course outlines 
of all the English classes stressed the provision of four English communication skills (listening, speaking, reading, and writing) so that students would be able to communicate in English after graduation. Since the textbooks used by both schools integrated all four communication skills, tasks pertaining to individual skills were not clearly separated from the rest. This, combined with the schools' syllabus, made it difficult for English lecturers to separate the lessons into skill-based units. What is more, the two schools were in the process of applying the communicative language teaching approach more radically in English teaching. This was evident from the addition of speaking tests alongside the traditional written tests as compulsory parts of evaluation. Speaking skills were tested only once at the end of the course and the tests lasted about 5-10 minutes. Both schools have made efforts to innovate how English is taught and improve students' English competency. Similar to many other institutions, those two schools did not officially apply the English output standard for their graduate students, despite the government's decisions regulating the standard English competency outcomes for graduate students. This was because the regulated outcome of B1-CEFR was too high; thus, if it were put into a real effect, a majority of students would not be able to graduate as they would be unable to achieve that level.

\subsubsection{Lecturer and Student Participants}

Lecturers from the two colleges were invited to take part in this study. The five lecturers (two in school A and three in school B), chosen by purposive sampling (see 3.4), already had Bachelor's degrees and four of them had also completed Master levels in the English language and/or Linguistics. The lecturers had spent from 7-12 years teaching English and had considerable experience working with non-English major students. In 2013, all the English lecturers at colleges in the city were assessed in terms of English language proficiency. All the five lecturers had achieved C1-CEFR (Vietnamese version) to qualify for teaching English at colleges (Vietnamese Government, 2010). However, to use English effectively in their EFL classrooms, the required English proficiency level is insufficient, and teachers also need pedagogical knowledge of how language can be used to support students' learning (Pham, 2018). In acknowledgment of this, the Vietnamese Ministry of Education and Training has introduced large-scale English-for-teaching methodology training programs for teachers, but none of the lecturers in this study had participated in this training. This could be because the programme had prioritised primary, secondary, and high school EFL teachers. Although all the lecturers said they had attended training workshops and local conferences in English language teaching methods at least once, and two lecturers had TESOL training and certificates provided 
by an Australian registered training organisation in Vietnam, it can be said that the lecturers in this study had few opportunities to engage with ongoing professional development activities. The five lecturers were all female, which reflects the reality that teaching English is a popular career for Vietnamese women.

The student participants were mostly studying in their first and second years and in different training majors. They came from diverse geographical backgrounds, including remote, highland, and the city areas. This created differences in the English language backgrounds and levels among students because in most remote or highland regions pupils begin to learn English 3-4 years later than those in big cities. Also, students from cities normally have better opportunities to practice and learn English outside classes; hence, their English levels could be much higher. Overall, the classes tend to reflect a wide range of English language proficiencies. The students' English levels were divided into beginning, lower-intermediate, and intermediate proficiency. Most of the students were at very basic English proficiency levels (beginning and lower-intermediate). The participating students for observation and questionnaires were selected by the lecturers who based their selection on the criteria I gave them (see Table 3.2). Participants for student focus group interviews were purposively chosen based on the selection survey at the end of the questionnaire (see section 3.4).

Table 3.1 summarises the information on the training sectors, English syllabus, lecturers, and students of the two research sites.

Table 3.1

Description of the Two Colleges

\begin{tabular}{|c|c|c|c|}
\hline \multicolumn{2}{|c|}{ Major sectors of education } & $\begin{array}{l}\text { College A } \\
\text { Information technology }\end{array}$ & $\begin{array}{l}\text { College B } \\
\text { Tourism } \\
\end{array}$ \\
\hline \multirow[t]{2}{*}{$\begin{array}{l}\text { Syllabi of } \\
\text { English }\end{array}$} & $\begin{array}{l}\text { Total of Modules and Periods } \\
\text { (Each period lasts } 50 \\
\text { minutes) }\end{array}$ & $\begin{array}{l}\text { Four modules } \\
165 \text { periods }\end{array}$ & $\begin{array}{l}\text { Three modules } \\
240 \text { periods }\end{array}$ \\
\hline & Programmes & $\begin{array}{l}\text { General English ( } 135 \text { periods) } \\
\text { English for specific purpose ( } 30 \\
\text { periods) }\end{array}$ & $\begin{array}{l}\text { General English }(150 \text { periods }) \\
\text { English for specific purpose } \\
(90 \text { periods })\end{array}$ \\
\hline \multirow{3}{*}{$\begin{array}{l}\text { Lecturers } \\
\text { of English }\end{array}$} & Number & Five & Six \\
\hline & Years of teaching experience & $5-10$ years & $3-20$ years \\
\hline & $\begin{array}{l}\text { Number of lecturers with } \\
\text { abroad education/ training }\end{array}$ & One (For 6 months) & One (For 1 year) \\
\hline \multirow{2}{*}{$\begin{array}{l}\text { Students of } \\
\text { English }\end{array}$} & Geographical background & $\begin{array}{l}\text { Cities } \\
\text { Remote } \\
\text { Highland areas }\end{array}$ & $\begin{array}{l}\text { Cities } \\
\text { Remote } \\
\text { Highland areas }\end{array}$ \\
\hline & Level of English & $\begin{array}{l}\text { Basic } \\
\text { Lower-intermediate }\end{array}$ & $\begin{array}{l}\text { Basic } \\
\text { Lower-intermediate }\end{array}$ \\
\hline
\end{tabular}




\subsubsection{The English Classrooms}

There were about 25-35 students in each class. Blocks of classrooms were quite close to each other with classrooms sharing walls with others. All classrooms (including English ones) followed the same designs and were equipped with a big green board or white board, and a medium-sized TV screen or a projector with a desktop computer. Lecturers of English used LCDs or speakers to play audio files for listening tasks. They did not use any microphones or audio-supportive tools even for large classes. Most of time the lecturers stood at their desks/boards speaking using the desktop computer mouse to control the presentation slides. Students mostly sat in long rows with fixed desks, and they all faced the board. Occasionally, some lecturers asked students to rearrange the organisation of the class seats to a U-shape at the beginning of a lesson and return them to normal at the end of class.

\subsection{Participant Recruitment and Selection}

A purposeful sampling technique is used to identify and select individuals who are especially knowledgeable about or experienced in the study phenomenon (Creswell \& Plano-Clark, 2011). Purposeful selection was found to be appropriate for this case study research since it helps researchers to reach the best possible source that is most likely to share information that is rich and relevant to the issue under investigation (Barglowski, 2018). Hence, this type of sampling method allowed me to select specific individuals to potentially participate that would help me best answer and further understand the research questions. The following table describes the selection criteria of this study.

Table 3.2

Selection Criteria of Participants

\begin{tabular}{|c|c|c|c|}
\hline Participants & Lecturers & Students (For focus group) & $\begin{array}{l}\text { Classes (For observation } \\
\text { and questionnaire) }\end{array}$ \\
\hline No & $\begin{array}{l}\text { Six (Three lecturers from } \\
\text { each college) }\end{array}$ & $\begin{array}{l}\text { Six groups of six students } \\
\text { (One group for each } \\
\text { lecturer) }\end{array}$ & $\begin{array}{l}12 \text { (Two classes of each } \\
\text { lecturer) }\end{array}$ \\
\hline Selective method & Purposive & Purposive & Purposive \\
\hline Selection criteria & $\begin{array}{l}\text {-Giving informed consent } \\
\text { to participate } \\
\text {-Holding a degree in } \\
\text { Linguistics or the English } \\
\text { language } \\
\text {-Teaching English for non- } \\
\text { English major students for } \\
\text { more than } 5 \text { years }\end{array}$ & $\begin{array}{l}\text {-Giving informed consent } \\
\text { to participate } \\
\text {-Being in the classes that } \\
\text { are chosen for observation } \\
\text {-Being interested in English } \\
\text { speaking and listening } \\
\text { strategies } \\
\text {-Being able to articulate } \\
\text { their thoughts } \\
\text {-Being confident to raise } \\
\text { their voice in groups }\end{array}$ & $\begin{array}{l}\text {-Non-English major } \\
\text { classes } \\
\text {-At the most common } \\
\text { English proficiency } \\
\text { levels (not too high nor } \\
\text { too low) } \\
\text {-Classes where lecturers } \\
\text { typically use spoken } \\
\text { English }\end{array}$ \\
\hline
\end{tabular}


Face-to-face and email were used as the main means of contacting lecturer and student participants during the research. After I received permission from the schools to conduct my research, I gained access to the email addresses of all the English lecturers at the two colleges. With the permission from heads of the schools and English faculties, I sent an email to all the English lecturers explaining the purpose of the research. Included in the emails was also the selection survey (Appendix E) together with two yes-no questions and one rating scale, which were designed to help me choose suitable participants. I included a brief introduction about myself and the study in the first part of the invitation email followed by the survey. Three lecturers from each college who hold Bachelor's/Master's degrees in English language/ Linguistics; had at least five years of teaching non-English major students; and showed the highest interest rate in classroom English communication were selected, and subsequently received all relevant information that explained what participation in the study would entail. In doing this, I could find rich cases for in-depth data to obtain local rich understandings of their life experiences, as characterised by the pragmatic paradigm and purposive sampling. Since lecturers are those who best understand their teaching, they were invited to choose any two classes with different lessons/skills, and an average English level (not too high nor low) to be observed. All students from those observed classes then received the paper questionnaires. A selection survey was included at the end of the student questionnaires with three yes-no questions which were used to choose participants for focus group interviews. I initially explained to students that the focus group discussions would be conducted in Vietnamese to make sure that they would not hesitate to join due to language concerns.

A total of six English lecturers from the two schools were invited and five of them (two from school A and three from school B) responded and expressed their willingness to participate in this research. Ten classes with a total of 289 students were observed and a total of 257 student questionnaires were collected. The selection survey identified 25 suitable students who were then contacted via email and mobile phone about how the focus group would work for them. Students who were studying with the same English lecturer were placed in the same group and they arranged the meeting time that suited them all. Overall, 21 students volunteered to participate and together they formed five groups, one for each of the five lecturers involved in the study.

\subsection{Ethical Considerations}

I thoroughly considered issues of research ethics not only with regard to the process of data collecting, but also in terms of analysing the data and writing up the results (Creswell, 2013; 
Denscombe, 2007). Ethical approval was first granted (Approval number: 25461) by the Human Ethics Committee of Victoria University of Wellington (Appendix A). The next step was to obtain permission and voluntary agreement from the participants, as well as explaining to them what their participation would entail. I made sure the consent forms (Appendix B) and information sheets (Appendix C) were sent to participants and signed before the commencement of the pilot study and the main data collection.

In addition to providing research information and obtaining consent, some other ethical precautions were taken in relation to the data collection techniques and procedures. The first issue was protecting the privacy of individual participants and the identities of the research sites by using pseudonyms when reporting the results of the observation and interview data (Drew et al., 2008). The second aspect was to ensure that the benefits of the research would be maximised (Hays \& Singh, 2012). In this regard, I made it clear before collecting the data and selecting participants that one of the overarching objectives of the current research was to offer useful understandings and recommendations of the Vietnamese EFL classrooms that could improve the efficiency of EFL teaching and learning. Informing the participants of the research benefits was also to encourage the effective completion of the questionnaires and proactive participation in the observations and interviews (Adams \& Cox, 2008).

Along with the beneficial dimension, minimising harm and risks especially for student participants was also considered to be important (Hay \& Singh, 2012; Stake, 2005). Because there were some instances when students had to talk about their lecturers, I made sure that the lecturers did not interfere while the students were completing the questionnaires nor knew the identities of the students joining the focus group interviews. This was achieved by my asking the lecturers to allocate me one entirely free lesson to work with students when I delivered and collected the questionnaires. I also emphasised to students that the "lecturers" in this research could be the current lecturer or any of those previously teaching them at the colleges; and their responses would be kept confidential and would not affect their academic results at school. To avoid causing any harm to the participants and schools due to being anxious, harmed, or misled, I clearly and honestly introduced myself as a $\mathrm{PhD}$ researcher and the research institution to which I belonged, provided my contact details, and shared the fact that I had approval from the heads of school for conducting the research.

Furthermore, a basic ethical principle for qualitative researchers is not to "tamper with the natural setting under study" (Drew et al., 2008, p. 19). I tried to minimise the influence of my presence by conducting nonparticipant observations, taking one short visit for each class to get 
the lecturers and students familiar with me, and choosing to sit in the back of the class where few students could see me. In this way, I encouraged the lecturers and students to behave normally as they do in their daily lessons. My position as a researcher, rather than colleague or some other work-related roles made it easy to for me to observe without intrusion and to prevent the ethical dimensions of teacher judgment (Mockler, 2014).

\subsection{Instruments for Data Collection}

This study was conducted using a qualitative-dominant mixed methods approach where quantitative findings were used to triangulate qualitative findings and increase their reliability (Creswell, 2013). These combined findings would generate meaningful information that contribute to the investigation of how lecturers were using English communication in nonEnglish major classrooms and to our understanding of how students and lecturers perceived different issues related to the choice of language and communication strategies. The three datagathering methods used for this study were classroom observations, questionnaires, and interviews, as summarised in the table below. I used part of my own individual experience and subjective judgment to compose the observation protocols as well as to design questionnaire and interview questions. This is suitable with my pragmatic research approach which allows me to choose methods, techniques, and procedures that best meet the research purposes (Creswell, 2013, Morgan, 2007).

\section{Table 3.3}

The Research Questions in Relation to the Data Collection and Analysis

\begin{tabular}{|c|c|c|c|c|}
\hline Research questions & Observation & Questionnaires & Interviews & $\begin{array}{c}\text { Data } \\
\text { obtained }\end{array}$ \\
\hline $\begin{array}{l}\text { 1. How much Vietnamese } \\
\text { versus English do lecturers } \\
\text { use? }\end{array}$ & $\sqrt{ }$ & & $\sqrt{ }$ & $\begin{array}{l}\text { Qualitative } \\
+ \text { quantitative data }\end{array}$ \\
\hline $\begin{array}{l}\text { 2. What English } \\
\text { communication strategies do } \\
\text { lecturers use and how are } \\
\text { they used? }\end{array}$ & $\sqrt{ }$ & $\sqrt{ }$ & $\sqrt{ }$ & Qualitative data \\
\hline $\begin{array}{l}\text { 3. What are lecturers' } \\
\text { perspectives on what affects } \\
\text { their English communication } \\
\text { in classes? }\end{array}$ & & $\sqrt{ }$ & $\sqrt{ }$ & $\begin{array}{l}\text { Qualitative } \\
+ \text { quantitative data }\end{array}$ \\
\hline $\begin{array}{l}\text { 4. What are students' } \\
\text { perspectives on what helps } \\
\text { them better understand their } \\
\text { lecturers' English speaking } \\
\text { and learn English more } \\
\text { effectively? }\end{array}$ & & $\sqrt{ }$ & $\sqrt{ }$ & $\begin{array}{l}\text { Qualitative } \\
+ \text { quantitative data }\end{array}$ \\
\hline
\end{tabular}




\subsubsection{Classroom Observation}

Observation is particularly used when "it becomes important to understand in detail a given phenomenon, while maintaining the environmental conditions in which it occurs" (Queirós et al., 2017, p. 376). Data gathered from classroom observation is authentic and reliable since it captures the full sense of the existing situations that take place during the actual teachinglearning process (Bryman, 2012). Observation enables researchers to document and reflect systematically upon classroom interactions and events, as they actually occur rather than as they are thought to occur (Marshall \& Rossman, 2014). When a researcher "relies partly on coming to know personally the activity and experience of the case", observation is the most meaningful data-gathering method (Stake, 2005, p. 4). Also, conducting observations involves "ongoing reflection on the settings and participants" (Hay \& Singh, 2012, p. 224). Observation data can be used to support the interview data as it can be used to confirm or critique what participants say in interviews or questionnaires.

However, several limitations and constraints of the observation method in educational research have been identified. For instance, Ornstein and Weiss (1991) argued that observations cannot measure all the teaching and learning behaviours, especially the complicated ones; thus, validity of data might not be obtained. However, this limitation was not a serious concern for the validity and reliability of the observation data in this study since I took careful fieldnotes and used data triangulation. Also, Stake (2010) mentioned that a novice researcher cannot make "an accurate record of what is happening ... failing to appreciate how much he or she has to know in order to edit the transcript" (p. 94). To address this problem, I applied cautious piloting to become familiar with observation procedures and practised taking notes to gain more experience in executing observation tasks (see 3.10).

In order to obtain relevant information in relation to the objectives of the study, I conducted two semi-structured, non-participant observations for each lecturer. Semi-structured observation combines broadly identified observational categories such as activities and mode of interaction with narrative fieldnotes and reflections (Riazi, 2016). This type of observation allowed me to take note of relevant things on the spot using a pre-planned observation protocol (Appendix F). Non-participant observation was chosen because I did not want to be involved in or affect the natural classroom activities (Bryman, 2012). I developed an observation protocol based on the reflection questions (Appendix M) as suggested by Hay and Singh (2012). Classroom observation aimed to describe lecturers' language choice and L2 communication strategies as well as students' responses to their lecturers' practices. 


\subsubsection{Questionnaires}

Qualitative data such as observations can be complemented with questionnaires, a "data collection technique that can gather any information and data" that are expected from respondents (Delsi \& Pupung, 2019, p. 47). By using questionnaires, researchers can measure many kinds of characteristics such as linguistic backgrounds and personal information (Johnson, 2014). In second language research, questionnaires can enable researchers to answer the questions systematically (Benali, 2013) and to find patterns in large-scale samples (Kendall, 2014). In this study, questionnaires were a good way for me to gather data about the personal and English backgrounds, as well as opinions and attitudes of hundreds of students. Moreover, using quantitative data collected from questionnaires was an effective triangulation tool which helped strengthen the qualitative data.

The questionnaires feature mainly closed questions because they are easy to administer, easily coded and analysed, allow comparisons and quantification, and are more likely to generate fully completed surveys while avoiding irrelevant answers (Hyman \& Sierra, 2016). The questionnaires were designed on the basis of multiple choice and Likert scale categories. Multiple choice responses can be quickly coded and synthesised to calculate frequencies of answers (Cohen et al., 2007); and scale-rating formats can be easy for respondents to use and generate a high completion rate (Chyung et al., 2018). To invite personal and honest comments from the participants rather just ticking boxes in the given choices, the questionnaires also included open-ended options for participants to share their own ideas if they wanted to do so. This kind of data could be a useful triangulation tool and source for the interview data analysis.

To meet the specific research aims of this study, I developed my own questionnaire survey, which was a difficult process as it involves developing and piloting the instrument prior to using it in my research (Roni et al., 2020). When composing the questionnaires, I referred to the research questions and aims, shortened the questions, diversified genres of questions to reduce boredom and random answering of participants, was thorough in using response categories for closed-ended questions, and asked my colleagues for feedback on the draft and final versions (Johnson, 2014). Also, I made sure to use familiar appropriate language to participants; avoid double, leading, or ambiguous questions; and piloted the questionnaires before releasing the final completed versions (Roni et al., 2020). When the questions are interpreted differently by the participants, it can lead to unreliable or invalid answers and responses (Kazi \& Khalid, 2012). Hence, the questionnaires were translated into the first 
language (Vietnamese) to make sure it was clear and comprehensible for all participants (see section 3.8 for the use of English and Vietnamese in data collection and analysis).

The questionnaire for lecturers (Appendix G) was composed to collect relevant background information such as degrees/qualifications, years of teaching experience, as well as information on their satisfaction and attitudes towards their English speaking. The questionnaire for lecturers was divided into two sections and collected answers via a 5-point scale and multiplechoice questions with one open-ended option at the end of each question. The questionnaire also included questions on lecturers' perspectives on the use of selected strategies as well as how objective and subjective aspects affected their use of English in non-English major classes. These questions employed a 5-point Likert scale (Wade, 2006) which required them to choose a number from 1 to 5 ( $1=$ Not a barrier to 5=Extreme barrier). The purpose of the lecturer questionnaires was to help me gain a better understanding of the five lecturers. These were analysed together with the interview data and provided context for that data. Findings from the lecturer questionnaire were treated qualitatively rather than quantitatively and analysed before the interviews were conducted.

The student questionnaire (Appendix $\mathrm{H}$ ) was constructed to collect information on the factors that encouraged and discouraged students' understanding of their lecturers' English speaking in classrooms. It was divided into three main sections, beginning with the personal and English learning background questions about gender, study majors, and years of learning English. The next part included six questions asking for general information such as their self-evaluation of their English competency, their satisfaction, difficulty, and motivation in relation to their lecturers' English speaking. These questions were mostly presented in a multiple-choice format and were based on the 5-point-Likert scale framework by Wade (2006). The last part had six questions focusing on students' perceptions of what afforded and hindered their comprehension of lecturers' English speaking, requiring students to label from 1 to 5 ( $1=$ Not a barrier to $5=$ Extreme barrier). A number of questions in the student questionnaire had open-ended options which "make a truer assessment of what the respondents really believe, and opening for unexpected or unanticipated answers" (Li et al., 2006, p. 438).

\subsubsection{Interviews}

I chose interviews as a data collection tool because this instrument broadens "the scope of understanding investigated phenomena, as it is a more naturalistic and less structured data collection tool" (Alshenqeeti, 2014, p. 40). I adopted a semi-structured style for the interviews 
because this provided flexibility and opportunities for unanticipated findings to be discovered, for mid-interview clarification, and for further information to be explored (O'Toole, 2010). When undertaking semi-structured interviews, I followed a checklist which "allows for indepth probing while permitting the interviewer to keep the interview within the parameters traced out by the aim of the study" (ibid, p. 39). I also used subjective judgment to design the interview questions. To ensure that the respondents would fully understand the questions and express their ideas clearly, the interviews were all conducted in Vietnamese. All interviews were audio-recorded to allow me to concentrate on the discussion and to later listen to the recordings repeatedly (Johnson, 2014). Two types of interviews were used for this study: focus group interviews with selected students and in-depth individual interviews with all the lecturers.

Focus group interviews were conducted with student participants because the "focus group methodology is useful in exploring what people think, how and why they think they way they do without pressuring them into making decisions" (Liamputtong, 2011, p. 5). The focus group approach involves students discussing questions with each other, with me merely acting as the facilitator, which is likely to be less intimidating than one-on-one interviews with a person of perceived higher power. As a result, students were less inhibited and spoke honestly, naturally, and confidently. As for the number of participants in each group, Denscombe (2007) suggests between six and nine participants because this "number allows for a fair range of opinions and experiences among the participants" (p. 181). Liamputtong (2011) considers that an ideal focus group should have between four and ten members. Following their ideas, I decided to aim to have six students in each focus group and one focus group for students of each lecturer sourced from two classes. Students were invited and chosen based on the selection survey that was included at the end of the student questionnaires (see section 3.4). Thus, a total of 30 students were invited to take part in five focus groups.

As for the lecturers, individual in-depth interviews were conducted. "At the root of in-depth interviewing is an interest in understanding the lived experience of other people and the meaning they make of that experience" (Seidman, 2006, p. 9). This means that individual indepth interviews were a suitable method to get a deep understanding of the lecturers' perceptions. From my experience and understanding about the psychology of lecturers, I found that the lecturers could feel more comfortable and freer to share their attitudes and perceptions if they only talked to me rather than in a group with their colleges. Also, an individual in-depth type of interview allowed me to focus on specific ideas with particular individuals 
(Denscombe, 2007); and to have the flexibility to probe, clarify, and check if the participants had understood my questions or if I had interpreted their meaning properly (Atkins \& Wallace, 2012). This flexibility further suggested that individual interviews were the most appropriate method to find answers to my research questions.

The next section provides a detailed description of the whole data collection procedure at the two research sites.

\subsection{Data Collection Procedures}

Data was collected during the second semester of the school year 2017-2018 (see Appendix D, for the collection procedure). However, the first and most important step was piloting the procedures, which is essential "to inform the researcher on what to expect during the study especially concerning the tools and instruments to be used, hence preparing for the study adequately" (Mwendwa, 2020, p. 23). Considering the foundational role of the piloting step, this section starts with a report on the pilot study before delving into the main data collection process.

\subsubsection{Piloting Report}

The objective of the pilot study was to increase the probability of success of the main study by identifying, and subsequently rectifying, some of the potential risks and pitfalls (Roni et al., 2020). In this study, I conducted the pilot to test and then amend the means of data collection, and to check the adequacy of the data collection method in answering the research questions of this study. All the final versions of the questionnaires, observation sheet, and interview protocols were translated into Vietnamese, followed by a translation checking process before being used in the pilot.

Piloting the Observations. Before travelling to the research sites, I made two observations in two evening EFL classes in Wellington, New Zealand. The classes were held for those seeking to apply for resident visas and to improve their English. The teacher was Vietnamese with about five years of teaching non-English major students in Vietnam. The learners had a similar profile to the potential participants in my study in that most of them were studying English as a foreign language, although they differed with regard to their English levels and ages. The first observation took about 50 minutes (12 learners) and the second (16 learners) about 47 minutes. Observing these two classes enabled me to familiarise myself with the observation and notetaking practices, which made me more aware of what kind of information should be included in the notes. This was when I noticed three main problems with 
the format of the original observation protocol. Firstly, the original form listed strategies in a row followed by the three frequency codes (Not observed at all; Some extent; Great extent). This made it really difficult for me to record the exact numbers of the strategies that were used, despite having three intervals for minutes 1-15; 16-30; and 31-45. Thus, amendments were made to the layout of the form to make it easier for me to capture the lecturers' significant behaviours. Secondly, I recognised that it was important to include time and duration spaces in the protocol as well (see Appendix F), which was helpful to keep track when referring to the audio recordings. Lastly, I took fieldnotes on a printed paper; but found that this was not a convenient way and it drew curiosity from some students who sat next to me: they kept casting their eyes on the papers. In response to this challenge, I changed my approach and started taking notes on my laptop while sitting in the back of the class to become less obtrusive and minimise the impact of my presence on the class procedures.

Before commencing the research study, I also conducted one final pilot in one EFL class (one lecturer and 25 students) in a college in Vietnam which shared a similar context with the research sites using the updated observation protocol to make sure all things would work smoothly. This practice helped me to consolidate my note-taking skills and taught me to notice a significant number of interesting unpredicted occurrences and behaviours what would become relevant to my research, such as humour and nonverbal behaviours. In the previous pilots, I had tried to take notes on every teacher-student interaction and as a result I missed some salient behaviours of the teachers related to the research aims. Also, I found that adding a timer to my recording device that exactly matched the lecturers' recorder and noting the time for important details were essential to track the instances in later audio reviews (see 3.7.2, for the use of classroom recording). This pilot stage really helped me refine my data collection techniques so that I was much more focused and skilful in notetaking for the main data collection.

Piloting the Questionnaires. As with classroom observation, piloting the questionnaire also underwent two phases. While still in New Zealand, I sent the questionnaires to 20 non-English major students who I had known and taught, using email and messenger tools. This phase enabled me to make several significant modifications to the language and question formats to make them clearer and more comprehensible to students. Firstly, a small number of students were confused about who "your lecturers" referred to and even showed concern about having to comment on the lecturer currently teaching them. Hence, I inserted a note in the beginning of the survey that clearly stated that the questions refereed to all those 
who had been teaching them English at the college where they were studying. This reassured them and made them more willing to share their ideas. Secondly, questions 6 and 7 regarding what motivated and demotivated them when listening to their lecturer speaking English had to be modified. Students opted for the same reason for both motivating and demotivating questions, which caused problems with the validity of the data analysis. To tackle this, I decided to integrate those two questions into one, starting with their feelings (motivated, demotivated, or neutral) first, followed by three sub-questions that specifically addressed each type of feeling. Additionally, the last two 5-point-Likert questions also needed to be reworded to make the statements clearer to students. The revised versions of the questionnaire were then distributed to the class of the last pilot site ( 25 students), and only some minor changes related to wording had to be made in response to the feedback provided.

As for the lecturer questionnaire, although it would be sent to only five lecturers and used in conjunction with the interview data, it was still piloted to check for its appropriateness. Five Vietnamese $\mathrm{PhD}$ students at Victoria University of Wellington who had been English lecturers in Vietnam and shared similar English teaching backgrounds with the target lecturers volunteered to test the questionnaires. Their feedback and replies allowed me to make some changes in the content of questions 10 and 11 so that they focused more on the communication strategies than teaching tools. Also, some statements in the last two 5-point-Likert scale questions were clarified to make them more comprehensible to the participants. Specially, the term "strategies" was clearly explained as English communication strategies to clearly differentiate it from general teaching strategies. This updated version was then given to the lecturer who taught the final trial observation class in Vietnam. This final trial round did not lead to many changes in the lecturer questionnaire.

Piloting the Interviews. The individual lecturer interview was in turn piloted with two Vietnamese English lecturers who were PhD students at Victoria University of Wellington. After the first interview (50 minutes), I revised the interview protocol and audio recording to check the quality of recording and the whole process of interviewing. I realised that it took me too much time to ask and explain the questions. The second interview (52 minutes) helped me realise that handling a basic checklist with key and probing questions was a useful asset because sometimes I was too engrossed in the conversation with lecturers and forgot to ask some key points. After the last pilot interview (55 minutes) which was done with a Vietnamese lecturer, I had mastered the interview procedures and developed the skill of asking for further information regarding unanticipated themes that were relevant for the research. This pilot phase 
helped me realise the importance of the first question, which serves to put interviewees at ease, and to check my understanding of participants' responses to ensure that the interviewees' perspectives were fully captured.

With regard to the focus group interview, one of the main goals for the pilot study was to ensure that questions were understood by the students involved in this research. Another goal was to make sure that the questions were successful in encouraging students to share insights that were relevant to answering the research questions. In the first stage, the focus group interview was trialled with 12 students in total (six participants and about 70 minutes for each group discussion) who were Vietnamese students (EFL learners) at Victoria University of Wellington, New Zealand. This phase allowed me to consider the answers and reactions of the participants in light of the intentions behind the questions, which helped me rephrase and modify discussion questions to obtain richer data. It also allowed me to self-assess my ability to conduct this tool effectively. Specifically, I improved my ability to encourage students to talk, for instance by using prompts such as tell me more or give me an example, and by giving all the members the opportunity to share their views. The second pilot phase (65 minutes) was conducted with a group of five students in the pilot observation class in Da Nang city. This step strengthened my skills as a moderator, especially in terms of my ability to respond to different situations that can occur during a group discussion.

\subsubsection{Main Data Collection}

Data were collected from 1 February - 31 March 2018. One month before the commencement, the heads of the two schools and respective English faculties were contacted via email with information about the research through written explanations to ask for their permission to access the sites and to work with their lecturers and students; and I received their permission. When I first came to the research sites, I handed a detailed research information sheet and consent form, written in Vietnamese, to all people involved and answered any questions they had. After ensuring everyone had understood the purpose of the study and the demands on individual participants, I obtained full written consent. Both the lecturers and students were provided with information on the confidentiality and anonymity of data, and the right of each participant to withdraw at any time.

I began with classroom observations. Each lecturer chose two of their classes for observation, each with a different skill level and a different focus. Thus, overall, ten classes were selected to be observed. Eight of them were at the starter level and two at the pre-intermediate level. 
Considering that the teaching context and the characteristics of learners should be taken into consideration when undertaking an observation (Jones \& Brownell, 2014), I talked with the lecturers before observations were made. This allowed me to gain a better understanding of the classes and students and offered me the opportunity to ask them for their advice on how to reduce the effect of my presence on the class in order to ensure the teaching and learning would happen in the most natural ways. Because teachers' instructional practices are not the same for all lessons (Kane et al., 2012), I made observations of two different lessons/skills (two consecutive periods for each) from two different classes and different weeks for each lecturer to ensure adequate and reliable data collection for analysis. I conducted ten classroom observations over a 4-week period with the five lecturers. Table 3.4 provides the contextual information about these observations (lecturers' pseudonyms, lesson skills/contents, the number of students, and the length of each observation).

\section{Table 3.4}

Classroom Observation Contextual Information (Ellen, Amy, Polly, Olive, and Una are lecturers' pseudonyms)

\begin{tabular}{lllll}
\hline Classes & Lecturers & Lesson skills/contents & $\begin{array}{l}\text { Number of } \\
\text { students }\end{array}$ & $\begin{array}{l}\text { Observation } \\
\text { duration }\end{array}$ \\
\hline Class 1 & Ellen & Grammar: Simple Present Affirmative & 30 & 87 mins \\
\hline Class 2 & Ellen & Reading: Legal Forms of Organisation & 21 & 79 mins \\
\hline Class 3 & Amy & Reading: Operating System & 19 & 61 mins \\
\hline Class 4 & Amy & Listening and speaking: Introducing people & 16 & 66 mins \\
\hline Class 5 & Polly & Speaking: Time expression & 23 & 36 mins \\
\hline Class 6 & Polly & Grammar: Question words & 31 & 32 mins \\
\hline Class 7 & Olive & Listening and speaking: In a restaurant & 32 & 65 mins \\
\hline Class 8 & Olive & Vocabulary and Grammar: Can vs Can't & 47 & 75 mins \\
\hline Class 9 & Una & Grammar: Past simple with ordinary verbs & 32 & 45 mins \\
\hline Class 10 & Una & Listening and speaking: We had a great time & 38 & 72 mins \\
\hline
\end{tabular}

The duration of observation for each class ranged from 32 to 87 minutes. Some lecturers asked me to stop the recording when the lessons moved solely to students' self-practice rather than teaching. That was why there were the different lengths of time recorded. One lecturer who said that a 30-minute observation would be enough for me to understand her teaching practice.

During each classroom observation, I put one small audio recorder on each lecturer's clothes (in the shirt pockets as informed in advance) to get the clearest sound. Meanwhile, on my desk was one other back-up recording device, whose timing was set simultaneously with the one attached to the lecturer. I used the semi-structured protocol that was devised for this study (Appendix F) to take notes on the lecturer's performances and students' general behaviours and reactions to their lecturers' English speaking. I operationalised fieldnotes and audio 
recording to supplement each other as the fieldnotes were used to write notes about details that would not be captured by the audio-recorded data, such as non-linguistic behaviours such as body language (Hennink et al., 2020). In other words, I used the fieldnotes to fill in the missing non-linguistic details of transcriptions of the observation data.

During the lessons, sounds from other classes and the outside activities was also recorded although some lecturers had closed the windows. Inside the classroom, sounds made by the electric ceiling fans made it challenging for me to hear lecturers' voice when they stood at the boards/desks speaking. The lecturers did not use any microphones or audio-supportive tools. The students mostly sat in long rows with fixed desks and they all faced the board. When the lecturers called on two students to model a conversation, they normally moved to the front of the board and faced the rest of the class; or they stood up at their desks and turned around to look at each other. When the lecturers raised questions, students normally waited until their names were called and only a few students raised their hands to give answers. All students stood up when they spoke to the lecturers, normally in Vietnamese even when the lecturers had used English.

Immediately after the classroom observations, I distributed the questionnaires. As advised by the lecturers, the questionnaires were distributed to the students in the period (last about 50 minutes) before the last observed lesson of each class. Paper questionnaires were found to be appropriate for the students because not all students could access the internet at home, which would likely result in a low response rate to an electronic questionnaire. Since I was present while students filled in the survey and set a time limit for students to complete the questionnaires, some common disadvantages of paper surveys could be avoided, including questionnaires being damaged during handling, or discarded and lost by respondents (Bucevska, 2007). Before handing out the papers, I explained that their answers would be confidential and not affect their school results. Meanwhile, I emphasised that the lecturers they had in mind when answering the questions could be either the one who was currently teaching them or other English lecturers who had taught them at the college. During the time students were completing the survey, their lecturer was not there. This reduced the pressure on students, created a relaxing atmosphere among the students, and ensured that they could respond to the questions in any way they wanted. I was there to give explanation when needed; but did not observe students' work. As for the five lecturers, I gave them their questionnaires when the last observation lessons were finished and collected their responses the following day. 
One week after the lecturer questionnaire was completed, I proceeded with the lecturer interviews. To ensure a relaxed atmosphere for the interviews, I interviewed each lecturer in the place where they desired. The interviews lasted from 50 to 80 minutes. I used the rough checklist with key and probing questions (Appendix I) as my main guide, which helped me make sure that all essential questions were covered. However, I tried not to depend too much on it to make the conversation more comfortable and natural. A small recording device and my mobile phone were used simultaneously to capture the interviews. At the end of the interviews, I also asked the lecturers' permission for coming back to them (either face to face or via email) if there was anything that was not clear or to check my interpretations of their comments. This led to a follow-up interview with one of the lecturers who was happy to talk to me for another 15 minutes to clarify some points from the previous interview.

Unlike lecturer interviews, focus group interviewing requires both skilful chairing by the interviewer and a physical set-up that is conducive to group conversations (Cohen et al., 2011). The pilot study prepared me well for my role as a moderator and group leader. It is important to ensure that students would know what to expect and what is expected of them (Breen, 2006). Hence, two days before the interviews, I emailed them to confirm the time and address as well as to explain the purposes and key contents to be discussed by students. I also noted that the discussion would be recorded and kept confidential to help them feel more confident and comfortable. One possible problem of focus group discussions is that some individuals may dominate the conversation (Sim \& Waterfield, 2019). To avoid this situation, I called on each participant for each question to make sure that everyone's ideas would be heard. They could also respond with "I had nothing to share/add" when it came to their turn. Also, I followed strictly the interview protocol (Appendix $\mathrm{J}$ ) in order to ensure that there was consistency across the various focus groups in the way that I treated them (Breen, 2006). The focus group interviews lasted between 60 to 90 minutes and were audio-recorded using one recording device and my mobile.

The next section presents the choices of language use and the procedures of data analysis.

\subsection{The Use of Vietnamese and English during Data Collection, Analysis, and Report}

Language is an important factor in research in that the results can significantly change when research is conducted using different languages (Abu-Shanab \& Nor, 2013). Hence, I was really conscious of choosing between Vietnamese and English during the data collection, analysis, and reporting processes, considering the participants' comfort and taking into account 
the most effective ways to elicit authentic and meaningful data that align with the research aims and methodology.

\subsubsection{Vietnamese for Data Collection and Coding}

Since it is important that participants feel comfortable throughout the data collection procedures, I decided to use Vietnamese - our shared first language - as a means of communication with participants. In addition to creating a sense of comfort, the use of the L1 also ensured that the participants would fully understand their roles as well as the research aims and questions. Using a shared language - Vietnamese - would accommodate our discussions "even in contexts where our differences existed" (Goitom, 2020, p. 557). More importantly, I was aware that, if I asked the lecturers to choose between English and Vietnamese as the interview language, some of them might opt to use English for reasons such as wanting to practise their English speaking or to "save face". However, using English as a foreign language might not allow participants, and especially students, to express "complex and sophisticated ideas, thoughts, and feelings that ... they wished to convey" (Li, 2011, p. 27). Thus, I decided to use Vietnamese, believing that it was the best language to help both students and lecturers express themselves freely and help me capture the "possible nuances of social knowledge and reality expressed in interviews" (Feldermann \& Hiebl, 2020, p. 250). Translating the instruments to the local language, in this case Vietnamese, can generate more reliable results as well (Abu-shanab \& Nor, 2013).

Once the data collection process was completed, the first task was to transcribe the interview data. I did all the transcription myself since the fact that I conducted the observations and interviews enabled me to include nonverbal aspects that no transcriptionist could (Hay \& Singh, 2012). I then sent the original transcripts to the respective interviewees to give them the opportunity to comment, withdraw an idea, or add more thoughts to the transcription document. All the five lecturers replied to me without any feedback, explaining that they were too busy to read the whole transcripts and that they trusted my transcription work.

The last major issue concerning me was whether to analyse the Vietnamese transcripts or their English versions, meaning all transcripts would have to be fully translated. Because Vietnamese is rich in dialects, proverbs, and particles that perform different grammatical functions or add different meanings to sentences (Ngo, 2020), no translation or interpretation can capture the original meanings as experienced by the participants. Hence, as a primary researcher with a deep knowledge and understanding of the Vietnamese language, I decided 
that coding the data in Vietnamese was the most effective way to maintain the authentic meanings and interpret the participants' intentions and viewpoints. This is supported by Nassar et al. (2011) who explained that keeping data analysis in the source language is a way to preserve the contextual aspect of data.

\subsubsection{English for Data Analysis and Report}

Once qualitative codes were established in Vietnamese and checked by a selected expert ${ }^{2}$ (see 3.9.1), I then translated the codes into English. After that, I started to develop, review, define, and name overarching ideas in English as this was the language that I needed use to discuss my research with my supervisors and to write up the findings. The selected expert in my study speaks Vietnamese as an L1 and had lived abroad for many years for postgraduate study and research. Thus, he could assure that my English translations of the codes and main findings were clear and adequately reflected the findings from the original Vietnamese transcripts.

The other issue to consider with regard to how to manage using two languages for data collection and reporting was the use of quotations from the interviews. The inclusion of quotations from interviews are a primary opportunity to present the collected data in an authentic way and provide a sense of "meaningfulness to the interpretive claims advanced by the authors" (Dai et al., 2019, p. 29). Accordingly, I decided to translate relevant excerpts that serve to illustrate findings for inclusion in the report, thereby enhancing the credibility and authenticity of the study (Messner et al., 2017). The remaining part of this section is dedicated to the issues of quotation translation.

\subsubsection{Translation of Quotations and Related Issues}

Quotes from interview data which are developed and collected "in one language do not reproduce perfectly in another language" (Humphrey \& Gendron, 2015, p. 53). In order to enhance the credibility of qualitative research studies, a proper translation of interview quotations is significant (Feldermann \& Hiebl, 2020). In fact, translators should not only know different aspects (syntax, morphology, dialects, metaphor, culture ...) of the source language but also systematically and accurately capture the full meaning and intentions of the interviewees (Al-Amer et al., 2015). They also need relevant knowledge and skills in the target language to produce the best interpretation and reconstruction of the source-language meaning.

\footnotetext{
${ }^{2}$ The expert here is a lecturer in Vietnam who holds a Doctoral degree. He helped me with checking coding and translation of codes and overarching findings from L1 to English.
} 
Being fully aware of this, I perceived that I should be the one who did the first translation for the quotations for three reasons. Firstly, I had personally talked, interacted, and established a relationship with the participants, which would help me define and explain some aspects related to their culture and "components of their identity" (Squires, 2008, p. 265). Secondly, I would be able to refer back to the participants if I needed further clarification, which would help me interpret what they really meant. Thirdly, I shared the dialect, culture, and a similar learning/teaching experience with them, which would further facilitate my interpretation.

However, the "language issue can be especially problematic for people whose writing skills in English are not as developed as in their native language" (Humphrey \& Gendron, 2015, p. 53). Even though I speak Vietnamese as an L1 and I have a high proficiency in English, I faced two challenges in trying to address how to translate some quotations from Vietnamese into English. The first difficulty was caused by the metaphorical concepts used by the participants. Since those concepts are culturally driven, it is difficult to find an equivalence that conveys the same idea in the English culture. For instance, one participant stated "không tưởng", which can be translated into "unrealistic", "unimaginable", or "a dream"; it can both convey a negative and positive meaning, depending on the attitude of the speaker. Hence, after I chose the best translated term, I returned the translated work back to the participant to verify if certain words and phrases that she used in her story were interpreted without losing the intended meaning (Bashiruddin, 2013). The second challenge was dealing with the contextual meaning. For example, the phrase "chạy theo giáo án" (focus on completing their teaching plans) is a common phrase and was used by some participants. A word-by-word translation would be to "run after teaching plans", but this interpretation would not make sense in English. Therefore, I got all the translated quotations checked by a Vietnamese teacher of English who had been working in New Zealand for many years. Her academic writing skills and experience in the practical use and culture of English and Vietnamese helped me tackle the translation-related issues and thus compose the best possible translations for the selected excerpts.

\subsection{Data Analysis}

This study employs a multi-analysis approach since it collects both quantitative and qualitative data (Johnson, 2014). Both types of data came from classroom observations, interviews, and questionnaires and the analysis of the data sets followed two stages: the first was very rapid and led to the main ideas and a tentative conclusion while the second came through writing about the key ideas in a recursive process which required referring back to the data and theory, using a critical lens and creative thinking as new ideas and concepts were established (Daniel 
\& Harland, 2017). With the pragmatic and case study approach, and the research aims, the analysis of data in this study was based on an open and flexible approach and triangulation of findings.

\subsubsection{Observation Data Analysis}

Firstly, I transcribed the audio-recordings of classroom observation to have a holistic picture of what had been observed. The transcriptions together with the fieldnotes on the observation protocol were used to look for the particular categories of the lecturers' behaviours and expressions. I used a checklist with descriptions of communication strategies to sort out all that were relevant to the lecturers' communication strategies and patterns. However, some categories that had not been listed in the protocol were also noted to have a complete picture of the lecturers' communication behaviours. I counted the frequency and identified the types of strategies before examining and describing each strategy in relation to its use in the lecturers' English speech.

Being aware that the communication strategy field was new and a challenge for me as a researcher, I got the final list of communication strategies with their types and functions checked by a peer who was interested in and knowledgeable about the field. This aimed to make sure that I had properly understood and classified the communication strategies. Sometimes, we came back to the fieldnote data to discuss and reach agreement on the use of some strategies, especially the nonverbal and humour categories. This peer debriefing section could have reduced my subjectivity, focused on correctness, and enhanced accuracy of research interpretations (Hendricks, 2006).

One aim of this study was to find out the balance between L1 and L2 use in the classroom. Because a turn might contain a long sentence or just one single word, both turns and words were used to analyse the amount of language use in Vietnamese (L1) and English (L2) (Storch \& Aldosari, 2010). To measure the amount of L1 versus L2 use, all L1 words/phrases were separated from the lecturers' speech in each task rehearsal and measured for time duration using the time count function in the transcription software.

\subsubsection{Interview Data Analysis}

I firstly transcribed the audio recordings (via https://transcribe.wreally.com/). Then I read through the entire dataset several times while listening to the recordings to make sure I would not miss any points. I started to have initial ideas by asking questions of the data. I took notes to save these early ideas in order to refer to them again later. I found this familiarisation step 
important since it enabled the remaining stages to produce more high-quality work (Terry et al., 2017).

After developing general ideas regarding the overall dataset, I began to produce codes, which helped label the segments of the data and simultaneously categorise and summarise each segment (Thornberg \& Charmaz, 2014). I tried to include all quotes, identified, and labelled "all segments of interest and relevance within the dataset, and everything that is of relevance within those segments" (Terry et al., 2017, p. 17). I coded the data using Microsoft Word's comment function and Microsoft Excel to tag and collate it. I compiled a list of codes that identified both patterning and diversity of relevant meaning within the data and collated data which was identified by the same codes.

I then took a closer look at the codes and combined, clustered, or collapsed them to form meaningful patterns. I thought carefully to identify similarities and relationships across a range of different codes to decide if they should be clustered together to make a key idea. I maintained my focus on the research questions to avoid getting lost in analysis and to keep the analysis relevant.

With the key ideas, I began to produce the report. This step involves a transition from "a purely analytic point in the research process ... to the bigger picture of the overall research" (Terry et al., 2017, p. 25). After checking if there was sufficient evidence to support all the key ideas included in the final selection, the key ideas were integrated into analysis and the literature to create an overall story and establish links to the research questions. I then sent the transcripts and key findings to the participants for them to check the content. Because all the lecturers did not want to read through all the transcripts, I then decided to provide the key findings (in the English language) to the lecturers so that they could check the language and add comments if they desired. As a result, one lecturer further explained why she used more Vietnamese during grammar lessons. One other lecturer asked me to change "moderately" to "slightly" for the finding on her satisfaction with the use of English in non-major classes, further giving me the reason for this. This member-checking phase enhanced validation of data since it helped corroborate findings as well as produce any further evidence which might not have given during initial data gathering (Yin, 2014).

Those who are new to qualitative research might face a common problem of inconsistency between the claimed theoretical framework and the actually presented analysis. For example, while novice researchers might claim their research has been informed by a social 
constructionism approach, the way they analyse their participants' words actually approaches language use from a realist perspective (Terry et al., 2017). To tackle this problem, I spent time reading the literature that applies the same theoretical framework used in my study and also looked at counter arguments to the usefulness of my approach. This helped me identify the ways writers "describe their participants' ideas and the claims they make from them" (p. 27), which further enhanced the consistency between the theoretical perspective underpinning this study and the analysis procedure.

\subsubsection{Questionnaire Data Analysis}

The student questionnaire, which was based on Likert scale, multiple choice, and open-ended formats, were analysed both quantitatively and qualitatively. Paper responses from 257 students were collected and coded on both: SPSS software version 21 and Microsoft Word 2010. All responses as well as the standard deviation were calculated. The p-value was found to be greater than 0.05 , which indicated no significant differences among both groups of students with regard to the statement presented. I described the characteristics of the data in quantitative forms such as frequency distribution and graphical displays (Johnson, 2014). Questionnaire items were tabulated as a percentage and count value to understand the meaning of the data gathered. The text responses for the open-ended questions were coded and treated as qualitative data, following a descriptive statistics procedure.

Since there were only five responses for lecturer questionnaires, they were mostly analysed in the margins of the hard copies. This kind of data helped me have general information of the five lecturers, which facilitated the following interviews with them. Thus, the lecturer questionnaire data were combined and reported together with the lecturer interview data.

\subsubsection{Integration of the Data Sets}

This study adopted a convergence parallel mixed methods research design. Thus, the integration of different data sets during analysis and report was believed to be strategic to produce more objective and valid results. In this research, the data integration was referred to as data triangulation, defined as "the use of two or more methods of data collection in the study of some aspect of human behaviour" (Cohen et al., 2011, p. 141). Data triangulation was an important step for this case study research because a case study can become strong and convincing if the findings fit well within the data sets (Jonsen \& Jehn, 2009).

In the first phase, all the data sets were analysed separately. After that, findings were triangulated. I listed the key findings from each instrument on the same page and considered if 
the findings agreed, offered complementary information on the same issue, or contradicted those from other instruments. When convergence was found on certain ideas, they were likely to be reported (e.g. students' low English proficiency level was perceived by both lecturers and students from questionnaires and interview data to hinder the use of classroom English). I also put an emphasis on the complementarity of the findings across the data sets. For instance, the focus group interview data furthered supported the observation data on the level of English and Vietnamese used by the lecturers, which seemed to strengthen the findings, especially when the lecturer interview data revealed a different story. With the purpose to reach in-depth information and have a deep understanding of the research phenomena, I also looked for disagreements between findings from different databases. For example, the discrepancies between what lecturers said in the interviews and what students perceived on the use of communication strategies were noted. As a result, the complexity and tensions between the lecturers and students were better demonstrated. With the three techniques for triangulating findings, it was believed that the research problems could be better examined to gain a more holistic picture.

The remaining sections of this methodology chapter discusses data validity, trustworthiness, and the potential limitations of the chosen methodology.

\subsection{Data Validity and Trustworthiness}

Validity refers to "the ability of a measuring instrument to measure the desired property" (Çalıklar \& Kadıŏlu, 2020, p. 54). Trustworthiness means that "the data analysis and the forthcoming results represent a plausible representation of the participants' explanations and exemplifications" (Daniëls et al., 2020, p. 8). When conducting a mixed methods study, researchers must ensure that it has "strong quantitative and qualitative validity" (Johnson, 2014, p. 309). With this qualitative-dominant study, I made an effort to minimise the threats to the validity of both quantitative and qualitative data and to enhance the trustworthiness of the qualitative data.

\subsubsection{Validity of Quantitative and Qualitative Data}

In this study, validating the questionnaires was given a special priority because they were specifically designed for the purpose of the research. To ensure the validity of quantitative data collection and analysis, I applied the three major steps as suggested by Delport and Roestenburg (2011) (Appendix L). I also adopted 15 principles recommended by Johnson (2014) when constructing questions (Appendix K) and used the peer-examination technique to 
check the draft versions (Creswell, 2013). Then, the questionnaires went through a two-phase pilot study (see 3.7.1) with students who shared many personal characteristics and contextual similarities with the potential participants of this study. Therefore, the threat to the validity of the survey instrument was reduced because the pilot and official practice data collection stage incorporated a standardised questionnaire that did not change much throughout the study (Creswell, 2014). During the data collection and analysis procedures, the characteristics and cultures of the participants and research sites where this research was to be carried were respected and accommodated. For example, paper questionnaires were used instead of an online survey because this better suited the circumstances of the student and lecturer participants. Also, I paid attention to the roles of careful sampling and appropriate statistical treatments of the data. All the above steps helped enhance the internal, external, content, ecological, and cultural validity of the quantitative data (Cohen et al., 2007).

With regard to the qualitative data, I followed three validity strategies to improve the validity. Firstly, data was collected using different methods such as questionnaires, classroom observations, and interviews. This approach adheres to the principle of triangulation of data sources and methods, the most commonly advocated method for enhancing research validity (Creswell, 2013; Merriam, 2009). Secondly, interview transcripts and key findings were sent to lecturers and students so that they could see if the data accurately reflected their intents and meanings, which belongs to the category of a member-checking technique (Creswell \& PlanoClark, 2011; Hay \& Singh, 2012). Lastly, the coding and developing themes were checked by a selected expert who had experience in communication strategies (see 3.9.1). This is referred to as the "external reliability" technique (Guest et al., 2012).

\subsubsection{The Issues of Trustworthiness}

Trustworthiness of this study was achieved through adhering to the requirements of credibility, dependability, confirmability, and transferability (Polit et al., 2012).

Firstly, credibility can be increased through prolonged field engagement (Denzin \& Lincoln, 1994; Morse, 2015). I spent a long time (nearly 2 years) designing this study, contacting the participants, getting to know and become familiar with the students, as well as collecting and coding the data. Hence, this prolonged engagement allowed me to establish a rapport and build trust with participants, understand their cultures and related contexts, which facilitated our later communication when I needed to check information about the data (Creswell, 2007). Also, member-checking where participants check the accuracy of the findings allows participants to 
contribute to the credibility of the information (Creswell, 2007; Creswell \& Poth, 2018). After the lecturers' perceptions were conceptualised into key ideas from the coded data, I discussed the ideas with the lecturers via email over a period of about six months.

Secondly, the dependability of this study can be established since "the research process is logical traceable and clearly documented" (Munn et al., 2014, p. 110). Thus, I followed proper data collection protocols in the observation and interview processes. In addition, to further increase dependability, I engaged in reflexivity practices (Darawsheh \& Stanley, 2014) by keeping a reflexive journal, which offered me the opportunity to reflect on and capture the reactions of participants and setting, how my personality might affect the research process, or notes about how data were collected and analysed.

Moreover, I tried to establish how clearly my interpretations and findings were developed from the data as a way to enhance confirmability (Nowell et al., 2017). Confirmability of this study was high through data triangulation, meaning data was derived from two different sources (qualitative and quantitative), people groups, and locations (Miles \& Huberman, 1994). Also, I held a peer debriefing session with two Vietnamese PhD students who were not involved in collecting or interpreting the interview data but shared the research interest. During the peer debriefing session, the process of data transcription and analysis and results were discussed. This practice helped me identify "any issues with the research including any errors, bias, or over- or under-emphasis on points" (Riedel et al., 2020, p. 65).

To enhance transferability of the results, I provided interview quotations and observation excerpts from the transcripts as samples of explicated meanings (Korstjens \& Moser, 2017; Patton, 2014). Also, rich and thick descriptions of the research process, setting, and details of the participants' selection and characteristics were applied to increase the transferability of the findings to other contexts. This will assist readers in understanding how or if findings may be transferable to other settings because of shared features across the sub-cases (Creswell, 2013). With the above effort to enhance the credibility, dependability, confirmability, and transferability of the data analysis and report, this thesis is believed to have a high level of trustworthiness.

\subsubsection{Potential Limitations in the Research Methodology}

Despite my efforts to build trust and confirm findings, I was still aware of some potential limitations in the research methodology and data collection methods. Firstly, the results of qualitative research might be affected by researchers' subjective consciousness (Anderson, 
2010), which could be a potential limitation for this qualitative-dominant study. Furthermore, I relied on individual experience and subjective judgement to design questionnaire and interview questions for the research. Hence, the preciseness of research was difficult to guarantee; and research results could not be totally objective and exact, despite different ways to reduce possible subjectivity and researcher bias (e.g. triangulation, member-checking, reflexive journal, prolonged engagement). Secondly, achieving true integration of the two types of data could be difficult since it "requires innovative thinking to move between different types of data and make meaningful links between them" (Tariq \& Woodman, 2013, p. 6). I addressed this challenge by continuously reflecting on the study's results and asking if my understanding had been enhanced by the combination of different data types.

Another possible limitation to the interview data could be the language barrier. The interviews were all conducted in Vietnamese and I am an EFL speaker. This might have resulted in some slight divergences from the intended meaning due to the need to translate the data. As a result, some of the research findings might not be rigorous, despite my effort to get my translation checked by one Vietnamese lecturer who had been working in an academic sector in a nativeEnglish speaking country. 


\section{Chapter 4. An Overall Picture of Lecturers' English Communication}

This chapter presents four overall aspects related to lecturers' English communication in EFL classes, which helps answer research questions 1, 3, and 4. It reports the results from the observations of 20 lessons, five student focus group interviews, five individual interviews and surveys with lecturers, and the questionnaire survey of 257 student participants. This chapter starts with reporting the level of English and Vietnamese spoken by the five lecturers in nonEnglish major classes. It then discusses lecturers' and students' perceptions of an English-only teaching practice. Next, the factors affecting lecturers' use of English in non-English major classes are described. The last section presents the factors which have an influence on nonEnglish major students' comprehension of lecturers' English speaking and on their learning motivation.

\subsection{Proportion of English and Vietnamese Spoken by Lecturers in Non-English Major} Classes

The first feature of lecturers' language use in the classroom that is considered here is the level of use of English and Vietnamese. The data presented in Figure 4.1 is based on observational data and captures the proportion of time (\%) the lecturers used Vietnamese and English in class. The names used for the lecturers are pseudonyms.

\section{Figure 4.1}

Percentages of Lecturers' Use of English (L2) versus Vietnamese (L1)

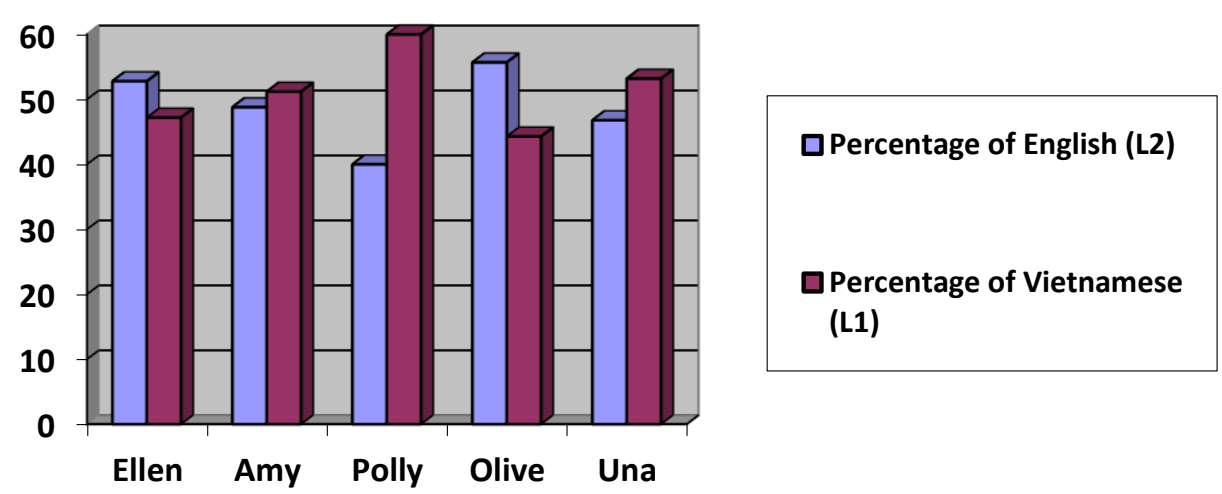

On average, the lecturers used English for $48.8 \%$ and Vietnamese for $51.2 \%$ of their total speaking time in class. The two lecturers in school A on average used slightly more English than the three lecturers in school B (50.8\% versus $47.5 \%)$. Three out of the five lecturers spent 
slightly more time speaking Vietnamese than English. One lecturer in particular, Polly, used Vietnamese one and a half times as much as English. This accords with data from the later interview where she stated that she mostly uses Vietnamese in class and that her Vietnameseuse can account for up to $70 \%$ of her total speaking time when she taught grammar lessons. In fact, among her four observed lessons, two were grammar lessons, which partially explains why she used much more Vietnamese than English.

The above finding further supports previous research (e.g. Al-Ghafri et al., 2019; Littlewood \& Yu, 2011; Liu et al., 2004). However, the proportion of English use observed in this investigation is significantly below those observed in other studies (e.g. Bozorgian \& Fallahpour, 2015; Taşçı \& Aksu Ataç, 2020). After the first phase (2008-2016), the National Foreign Languages Project 2020 continues with its new phase 2017-2025 (see 3.3.1). This new phase aims at an overall improvement of the quality of English teaching at all education levels (Government of Vietnam, 2017). These goals have posed new challenges for English educators and policy makers in the country. The two tertiary schools in this study encouraged maximal English use and aimed at developing communicative competency among students. However, like many other institutions, they did not implement any official policies on the language of classroom instruction; the lecturers themselves could decide how English was best used in their EFL classes. This might have led to the difference in the levels of L1 and L2 use across the lecturers as seen in Figure 4.1.

In accordance with the observation results, the students' perception was that there was a slight difference in the balance between English and Vietnamese used by lecturers in classes, with slightly more Vietnamese used than English. About half of the total 21 interviewed students felt that their lecturers used more Vietnamese than English. For instance, IS2 said: "I am quite attentive to the teaching of my lecturers in class and find they still use ... more Vietnamese; I can estimate about 70\% Vietnamese and 30\% English". Another student from a different class and school, TS3, shared: "Yeah, my lecturer mostly speaks 60\% Vietnamese and 40\% English". Two other students, TS9 and TS8, both asserted that there was an equal amount between English and Vietnamese used by their lecturers. Three students estimated that their lecturers spoke a bit more English (about 60\%) than Vietnamese. For example, IS3 stated: "In reality, English is used a little bit more than Vietnamese; the rate is about $60 \%$ English and $40 \%$ Vietnamese". However, more students thought that their lecturers were speaking more Vietnamese than English. 
From the lecturers' perspective, four out of five said that they normally used more English than Vietnamese, as Una and Ellen shared:

Normally, I speak a bit more English than Vietnamese. The ratio is about 70\% English. (Una) The ratio of English and Vietnamese I use depends on the taught levels, skills, and subjects. I usually use more English than Vietnamese. (Ellen)

As can be seen, most of the interviewed lecturers thought that they normally spoke more English than Vietnamese in their classes. However, the observation and student interview data suggest it was less than they thought. Some possible reasons for the discrepancy could be explained by the lecturers' basing their estimates on students' levels of abilities (Li \& Wu, 2017), the complex contexts of their teaching practices, or the difficulty level of the lessons (Shabir, 2017). Some other possible reasons will be presented in section 6.3.1.

In order to find out potential reasons for the lecturers' choices and practices of L1 and L2 use, it is helpful to examine the lecturers' and students' perspectives of an English-only approach in non-English major classes.

\subsection{Lecturers' and Students' Perceptions of an English-only Teaching Approach}

All the lecturers in this study supported an English-only approach and realised its benefits for both lecturers and students, explicitly expressing their strong desire for maximum L2 classroom practices. Here are some sample opinions:

Using 100\% English is ideal because it is the ultimate aim of English teaching. It benefits students' learning outputs and interests lecturers. (Polly)

There will be an authentic communication environment for both teachers and students in English-only classes. (Olive)

Polly and Olive both agreed that teaching English in English was an ultimate target because it creates an authentic environment that benefits students' communication ability. They also believed that the English-only instruction would increase lecturers' interest in their teaching. However, as can be seen from Ellen's and Una's comments, the common perception among the lecturers was that an English-only practice in their classes was unrealistic or inapplicable:

I totally support it [an English-only approach] and that is what I want. The more English to be used, the better. But I do not think it works smoothly. (Ellen)

I also like this practice [English-only], but I am afraid that it is not suitable for students' proficiency levels. (Una) 
The above extracts show that the lecturers fully supported the idea of the English-only instruction. Yet, they did not believe in its effectiveness when being applied into real practices for reasons that are exemplified by the following excerpts from their interviews:

It would be perfect if English can be used up to the maximum level, but as you may know, students' English levels are too low for us to apply English-only teaching successfully. Our students will be stressed. (Ellen)

This [English-only policy] sounds good but it will not be effective with current students' proficiency level. Some of them cannot say a simple English sentence correctly and their listening skills are really poor. They may be shocked and stop attending classes. (Polly)

Most students are familiar with the method of bilingual teaching from previous classes and are not willing to welcome new teaching practices. Therefore, it is very difficult to apply the monolingual approach in non-English major classes. (Amy)

Students' limited comprehension level leading to their negative reactions when lecturers used English entirely was identified to be the biggest problem to English-only teaching, as stated by Ellen and Polly. They were worried that using English only might cause students to feel stressed, shocked, and stop attending classes. From Amy's perspective, it would be difficult to make students adapted with English-only classes because they had not encountered this approach before. The above-mentioned factors were perceived to be barriers to the application of English-only practice. Further and deeper discussions on other factors affecting lecturers' use of English in non-major classes that may shed further light on lecturers' cautious attitude toward an English-only approach will be presented in 4.3.

As can be seen from the comments, even though all lecturer participants supported a monolingual approach in class, they effectively applied a bilingual method in practice. This tension could have been caused by the lecturers' perception that this was impossible due to students' limited English proficiency, which aligns with previous research (e.g. Beckett \& Li, 2012; Chapple, 2015; Ekoç, 2020; Macaro et al., 2018). Further discussion about the tension between lecturers' perceptions and practices will be found in section 6.3.

Although most of the lecturers did not think it was possible to successfully apply English immersion practice in their classrooms, they shared their views on the conditions that are required for this practice to work. Firstly, Amy, who believed that this practice was impossible and would not be successful, expressed the view that native-English teachers should be invited for low level proficiency classes to make better progress. Her suggestion indirectly supported the effectiveness of English-only teaching practice, since it is likely that native Englishspeaking teachers do not speak Vietnamese and hence use only L2 to speak and communicate in classes. Secondly, Ellen said that everything started from habits; if teachers used Vietnamese 
right from the beginning of the course, then students would remain comfortable with Vietnamese. She added that once an English-only policy was applied right from the beginning with really simple language, both students and lecturers would be in the habit of using L2 in class, which would ensure its success. Lastly, Olive added: "Once solutions are found to tackle the problem of helping students better understand lecturers' English speaking, English-only teaching will come into an effective practice". Here she voiced the perception that Englishonly instruction classes could work if effective strategies that would assist students to better comprehend English speaking were applied. The comments presented above demonstrate the complexity of lecturers' perceptions regarding the use of English as the medium of instruction in EFL education, which aligns with research (e.g. Awaiko Westin, 2019; Cheng, 2017; Simbolon et al., 2020).

As for students, only five of 21 interviewed said that they totally supported English-only teaching practice. Eight students voiced their support for an English-dominant approach and the others did not have ideas on this issue. Here are their opinions:

English classes are the only environment where students have opportunities to listen to and use English, so lecturers and students should take this opportunity. (IS1)

When learning English, students should have the right to listen to and speak English entirely; listening to lecturers speak English helps us find out our mistakes and learn how to speak English and use it properly. (TS4)

It is useful when lecturers can speak a lot of English; but not 100\%. I think we still need them $10 \%$ for explaining really difficult words. (IS2)

I do not think my teacher can entirely use English to teach us because we will not be able to understand them. But they really need to speak English predominantly to help us improve our English listening. (TS14)

IS1's and TS4's comments suggest that they considered the language classroom to be the main place where they could receive English input and perform output. Typically, they believed that English-only teaching would help them identify their language gaps and improve their performances. Similarly, IS2 and TS4 thought that lecturers' maximal English use was useful for them to improve their English listening skill. The students did not totally favour the Englishonly practice but support an English-dominant approach since they still worried about not being able to understand all the English spoken by their lecturers. Those findings further support previous studies that showed learners had both positive (e.g. Bukve, 2020; Uçar \& Soruç, 2018) and negative attitudes (e.g. Doiz et al., 2012; Lee \& Lo, 2017) towards the implementation of an English-only approach in EFL classrooms. 
In examining the lecturers' use of classroom English, besides their own practices, it is equally important to explore the factors that affect their decisions on how English is used in nonEnglish major classes.

\subsection{Lecturers' Use of English Communication}

\subsubsection{Student-Related Factors}

Students' Proficiency Levels. As mentioned in section 4.1, the lecturers' perceptions of students' proficiency levels affected their decisions on the amount of English and Vietnamese to be used as a language of instruction and classroom communication. The lecturers claimed that the higher the English proficiency level of their students, the more English they used.

In the questionnaires, when labelling the factors which influenced their use of English in teaching, all the lecturers also mentioned students' low level of English proficiency and comprehension as extreme barriers. This is strengthened by the interview data, as illustrated by the following extracts:

The main problem arises from students whose pronunciation, vocabulary, grammar, and communication skills are really low. I have difficulties using English to teach them because making them understand my English requires a lot of effort and techniques. (Ellen)

The proficiency of non-English major students is much lower than that of the majors, leading to a lot of difficulties in making sure they will all comprehend my English. That is the main problem! (Olive)

When students do not understand although I try to help them, I feel disappointed and do not want to use a lot of English anymore. (Polly)

Ellen and Olive identified the competence level of students to be the main problem to their using a lot of English in non-major classes. Students' limited comprehension ability particularly challenged the lecturers' ability to use English as the sole medium of instruction. Moreover, when Polly's efforts to improve students' comprehension was ineffective, she would feel demotivated in trying to use it in future practices. Students' limited comprehension also affects the lecturers' satisfaction and feelings toward speaking English in class. This is supported by Pourtoussi et al. (2018), who argued that student-related factors had the highest influence on motivating or demotivating teachers.

What is more, a range of proficiency levels within one group or class made it difficult for some of the lecturers to use a lot of English:

The second challenge is consistency. Students come from many different regions; in some areas, children begin to learn English at grade 6, while in other regions, children do not study 
English until they enter grade 10. The learning and teaching conditions in different places vary as well. Since students' levels and English background are heterogeneous within one class/group, lecturers do not know the amount and effective ways to use English in those classes. (Amy)

Amy realised that students came from different regions where they have been taught English for a different number of years using different teaching approaches, which led to heterogeneous proficiency levels within one class. This, she thought would make it difficult for lecturers to decide how to use English in those classes as some students would struggle to understand lecturers' English while at the same time others would think the level was too basic and simple for them. Students' heterogeneous English proficiency levels was addressed by $\mathrm{Li}$ and $\mathrm{Wu}$ (2017) as the main concern for teachers because it forces them to spend time planning different activities to match students' varying proficiency levels. Students' low English proficiency has been shown to hinder the success of English medium instruction (Ekoç, 2020; Macaro et al., 2018). In this study, students' proficiency levels were perceived by the lecturers to affect both the quantity and quality of their English.

Students' Learning Habits and Motivations. Answering questionnaire items about perceived barriers to their English speaking in non-English major classes, three lecturers listed the habit of using Vietnamese amongst students as the most likely barrier. Some lecturers elaborated on this point in the interviews:

Students are reluctant to speak English. Sometimes they know how to say things in English, but they just sit in their group and all together say: “Teacher, speak Vietnamese, please!". They still answer in Vietnamese out of habit when I speak English to them. They do not use English until I get angry. (Amy)

Many students can speak English but only when I urge or force them to do so. (Una)

Sometimes I show them some simple responses in English such as how to reply to a question about age or how to do self-introductions and I ask them just to repeat them, but they refuse to do so. It seems that they prefer using Vietnamese. I used to be frustrated and even did not want to speak English in those classes anymore. (Ellen)

According to Amy, the fact that some students did not respond to her in English was not only because they could not, but because they were too shy and fearful to speak English. Amy experienced situations where students refused to perform in English although they were able to do so. Similarly, Una shared that a lot of her students postponed speaking English until she pushed them. Ellen's students sometimes did not respond to her in English, preferring Vietnamese instead, which she found frustrating and demotivating to such an extent that she was reluctant to continue to speak English in class. Students' reticence to use English in class is exemplified by Amy's story of a whole group of students chorally insisting that their lecturers 
used L1 instead of L2. I also noticed students' habit of using L1 during classroom observations when some students were reluctant to respond to their lecturers in English until they were asked as illustrated in the excerpts below that were taken from my classroom observation protocols. These sorts of exchanges were in fact quite common in most of the observed classes.

Excerpt 1: (Observation class 1 - Amy)

Lecturer: Skim the text and tell me what is it about as quickly as possible. Các em có 10 phút để chuẩn bị [You have 10 minutes to prepare]

Students: Nói tiếng Việt đi cô. Cho nhanh [Speak Vietnamese, teacher. It is quicker]

Lecturer: Các em sẽ phải đọc lướt bài đọc thật nhanh và nêu ý chính của bài. [You will have to skim the text quickly and tell me the main ideas of the reading text]

Excerpt 2: (Observation class 2 - Olive)

Lecturer: Longer and final sound (pause) so must be now say the second time "he can't" and (pause) another way another thing this is "at all". This is in the negative way

Students: Cô ơi, giải thích bằng tiếng Việt cho dễ hiểu cô [Teacher, please explain it in Vietnamese so that it is easier to understand]

Lecturer: Ok. Phải phát âm dài hơn và chú ý âm cuối. Ở phủ định ta nói “can't" hoặc "at all" [You must pronounce with a longer sound and pay attention to the final sound. In the negative, we say "can't" or "at all"]

After Amy gave the direction to do the reading comprehension activity in English, the students immediately asked her to switch to Vietnamese. As a result, Amy immediately translated her direction into Vietnamese. In Olive's class, she explained how to pronounce can't in English. The students then required her to explain it again in Vietnamese for better comprehension, which she did. This shows that students' habit of using L1 and negative reactions to lecturers' use of L2 were likely to negatively affect the lecturers' efforts to keep insisting on the use of L2. As a result, the lecturers immediately switched to L1 without trying other ways to make students understand them using L2.

Students' low learning motivations and practical study purposes were also perceived to be important hindrances:

Because English is not the main subject, non-English majors are not highly motivated learners. They study just enough to pass or just to get enough grades and have no high motivation for self-study and putting in any effort. Thus, lecturers are not motivated to help them understand their English. (Ellen)

The majority of non-English major students are often not aware of the important role and benefits of English in this era. Their lack of motivation affects our teaching; we are not really satisfied because we cannot fully implement the monolingual method as we have always intended and desired to. (Amy)

From Ellen's teaching experience, she felt that non-English major students had low learning motivation; an impression which was supported by their pragmatic approaches to study such 
as studying just to get enough points to pass the subject. She added that students' low motivation also had a negative effect on lecturers' efforts to get them to understand their spoken English. As for Amy, she expressed the view that non-English major students often lacked motivation to learn English, which caused lecturers' feeling unsatisfied with their featuring because they could not successfully implement their plans and pedagogical intentions into practice. In this study, the lecturers perceived students' negative learning habits and their own resulting demotivation as barriers to their speaking English in class, which was then thought to affect their teaching motivation. The above findings are supported by other researchers (e.g. Aydin, 2012; Javadi \& Mohammad, 2014) who also argued that low motivational level, lack of interest, or disruptive behaviour of students can have significant effects on teacher motivation.

Concern for and Understanding about Students. From the interview data, it can be seen that the lecturers' motivation to speak English was closely related to their concern for students, which falls into the affective domain. For example, when answering why she felt motivated to speak English in low-level classes, Olive noted: "I see students are trying to learn English for their future jobs". Amy had similar ideas: "Some of my students are eager to listen and respond to my English speaking”. These comments show that students were an important source of motivation for the lecturers to keep speaking English, despite some other barriers. That students are motives for teachers was also noted in previous research (e.g. Pourtoussi et al., 2018; Sugino, 2010).

According to the questionnaire results, lack of understanding about students at the interpersonal level was believed by all the lecturers to be an extreme barrier to their use of English as the medium of teaching and communication in non-English major classes. This issue was also raised in the interviews:

Perhaps since I normally teach each class for one semester, I do not have enough time to understand students' levels, habits, and aspirations. I do not know whether I should speak English more or less; faster or more slowly. (Polly)

If lecturers have a better understanding of students' aspirations regarding the use of English, they will be more confident and can better prepare for it. (Amy)

Polly thought that her lack of understanding about students was due to the regular rotations of lecturers in charge of one class, which caused inadequate time for her to get a better understanding of different students' needs. As a result, she did not know what students expected her to do regarding the amount or speed of English speaking in those classes. Amy reported that her use of English would be more effective because she would be better-prepared 
for her English speech and then perform more confidently with a better understanding about the students' learning goals. Findings illustrate that the lecturers' pedagogical use of English was affected by their level of understanding of the students' language goals. In line with empirical research (Gibbons, 2019; Smit et al., 2014) which states that understanding is an important element in positive teacher-student relationship, the above finding of this study confirms the direct influence that knowing about the students has on teachers' L2 use.

Some lecturers reported that class placement test results and students' academic reports for previous courses were the main information sources for them to get to know more about their students. However, those tests and student records reflected only students' general English backgrounds and grades; they did not show details about students' personal expectations or preferences for how English was best used and taught by lecturers. These findings demonstrate lecturers' pedagogical decisions and practices of using English in the classroom are strongly affected by different student-related aspects. These issues will be further discussed in sections 6.2.1 and 6.2.2.

\subsubsection{Lecturer-Related Factors}

The lecturers also reported that some factors related to themselves affected their use of English in non-English major classes.

In the survey data, two lecturers indicated that one of the main reasons they felt motivated to speak English in non-English major classes was It is a chance for me to maintain my Englishspeaking skill (Amy, Una). One important motive for non-native L2 teachers to use L2 in teaching was to maintain their own English language competency, as a part of professional development. Because those lecturers worked in a non-immersion setting, their opportunities to use English were also scarce. Furthermore, it appeared that overloaded teaching classes and domestic responsibilities meant that particularly married lecturers with children have few opportunities to practice and improve their English skills outside classroom time. In fact, most of teachers of English in Vietnam are EFL speakers, who may have different linguistic deficits, and struggle for example with vocabulary, fluency, or pronunciation (Riordan, 2018b). This is because those EFL speaking teachers typically work in non-immersion settings where access to the target language and culture is so scarce that they have few opportunities to practice and improve their own English skills, especially speaking. Hence, English classes were perceived by those lecturers to be a key setting for them to use and maintain their English fluency. 
In the survey, four lecturers also indicated that the biggest problem hindering their use of classroom English was their own lack of practical skills and strategies to help them make students better understand their English speaking. This is further supported by the lecturer interview data. For instance, during the interview Amy shared: "I still feel unconfident because I do not have much knowledge about teaching strategies, especially the most effective ways to make oral presentation in English". In the same vein, Polly felt that she was not good at and wanted to be trained in English presentation skills so that students could better understand her English speech. This means that both knowledge deficiency of communication strategies and lack of skills in presenting in English to students were perceived by the lecturers to reduce the effectiveness of their use of English to teach and communicate with non-major students.

Lastly, three of the five interviewed lecturers felt that their personal characteristics also contributed to the ineffectiveness of their classroom English use. Amy realised: "I lack selfconfidence and sometimes make uncontrolled grammatical mistakes when speaking a lot of English". Una shared the same worry about making mistakes because of having to constantly translate and code-switch to Vietnamese when speaking English while she did not have a good control on her speaking. She stressed, however, that the sole use of English would make her English-speaking smoother and minimise mistakes. This indicates that the lecturers' worries about making mistakes when speaking English affected their willingness to use English. As for Olive, she found it difficult to use gestures or nonverbal means when speaking, elaborating: "I think it is probably due to my personality. I also tried using them for several times but found it too difficult". This means that lecturers' personal characteristics might have an influence on their use of English communication strategies, which seems to be an under-researched issue in the field.

\subsubsection{Contextual Factors}

Lesson Skills/Contents. When asked to describe situations when they feel they cannot use a lot of English, Una and Ellen offered the following:

How much English I use depends on skills; when teaching grammar, for example, I will speak more Vietnamese so that students can understand me better. (Una)

For grammatical lessons, I use more Vietnamese because I need to explain the rules carefully. As for other skills such as vocabulary, pronunciation, or reading comprehension, I can use English more easily. (Ellen)

The above interview data illustrate that the lecturers' classroom language choices and the amount of English use depends significantly upon the lesson type and skills to be taught. The 
two lecturers both commented that they usually spoke more Vietnamese when teaching grammar and language structures compared with other skills. The main reason, as revealed by Ellen, was to save time: "even Vietnamese explanations need several repetitions before students can understand, let alone saying the rules in English, which takes a lot of time". Another reason why some lecturers used Vietnamese when teaching grammar was that they expected all students could understand their explanations and the lessons. This notion is reflected in the following statement by Polly: "All lecturers expect their students to understand all the functions and uses of English structures, so L1 is the only option". This data support other work finding that explaining grammar using the L1 is more efficient and ensures learner understanding (Cook, 2001). EFL teachers' tendency to use L1 more frequently during grammar lessons has also been found in other studies (see Alijani \& Barjesteh, 2018; Arshad et al. 2015, for more).

The level of difficulty of the content was also perceived to be a factor which decided on how much English lecturers used:

The second factor that affects my language choice is the complexity of the lesson content, which influences the difficulty level of the speech and message I want to convey. (Amy)

Amy did not think that her English use was based on the particular skills or types of a lesson. Instead, she believed that language choice was related to the lesson's content or the idea she was trying to convey.

The observation data support the lecturers' ideas. During grammar and reading lessons, most of the lecturers spoke much more Vietnamese than English, with the balance being around 70\% and $30 \%$ respectively. They used Vietnamese when translating grammatical rules (e.g. the usage of the present simple tense) so that all students could have a thorough understanding. When the lesson focused on listening and speaking or vocabulary there was a relatively equal ratio between $\mathrm{L} 2$ and $\mathrm{L} 1$ use. This might be because the lecturers seemed to want their students to fully understand the forms and structures, which tend to be the focus of tests and examinations. This issue will be further discussed in 6.2.1.

Class Size. Two lecturers said their use of English was also influenced by the number of students in the classes:

It is impossible to apply this method if a class is too large because the students often communicate with each other in Vietnamese. As for small size classes - about 20 students for example, I will closely monitor students and restrict their use of Vietnamese language, and encourage them to speak English more, but I cannot totally ban them from using Vietnamese. (Olive) 
As for small classes, with from 12 to 15 students for instance, it is possible for lecturers to practice English with each individual. It is easier for classroom management, and hence lecturers will have more time to speak English as well. (Una)

Olive's opinions are in line with Hadi and Arante (2015), who found that large class sizes influenced students' preference to use the local language. The lecturer's comment that an English-only approach would work only if class size was reduced is also reflected in Wei's (2013) conclusion: "English-only teaching is more suitable for small classes. It will offer teachers more chances to understand the challenges students confront, and students could have more chances to practice" (p. 197). Una believed that she would not have to spend a lot of time on classroom management tasks with smaller size classes; and would have more time to speak English to students. In large classes, teachers have to deal with a lot of classroom management tasks which normally takes time and makes teachers use students' L1 (Cook, 2001). Maybe it is because of this reason that the two lecturers believed teachers' quality and quantity of English speaking would improve if there were fewer students within one class.

The observation data show that the number of students in each class of school A was smaller than in school B. On average, each class in the former consisted of 20 to 30 students while the number of students in one group at the latter ranged from 30 to 40. Results suggest that the lecturers in school A used English more on average compared to the lecturers from school B (see 4.1). This indicates that the bigger a class is, the less English and more Vietnamese lecturers tend to use. This observation finding strengthens the lecturers' perceptions that class size affected their use of English.

In addition to the lecturers' practices and perceptions, it is equally important to consider what affected the students' comprehension of their lecturers' English and their learning motivation in order to gain a better understanding of lecturers' classroom English use.

\subsection{Students' Comprehension of Lecturers' English and their Learning Motivation}

Before delving into what may have affected non-English major students' understanding of lecturers' English and their learning motivation, it is necessary to know about their perceived comprehension levels, listening motivation, and learning improvement.

\subsubsection{Students' Comprehension Levels, Perceptions on Learning Improvement, and Motivation to Listen to Lecturers' English}

This section discusses the answers from 257 participants to the survey questions Q.4: "Overall, how much do you understand your lecturers' English speaking?" and Q.5: "Overall, to what 
extent do you think your lecturers' spoken English in class helps you to improve your communication skills?". The participants' answers are illustrated in Figure 4.2.

\section{Figure 4.2}

How Much do Students Understand Lecturers' English Speaking and How Much does It Help Them Improve Communication Skills?

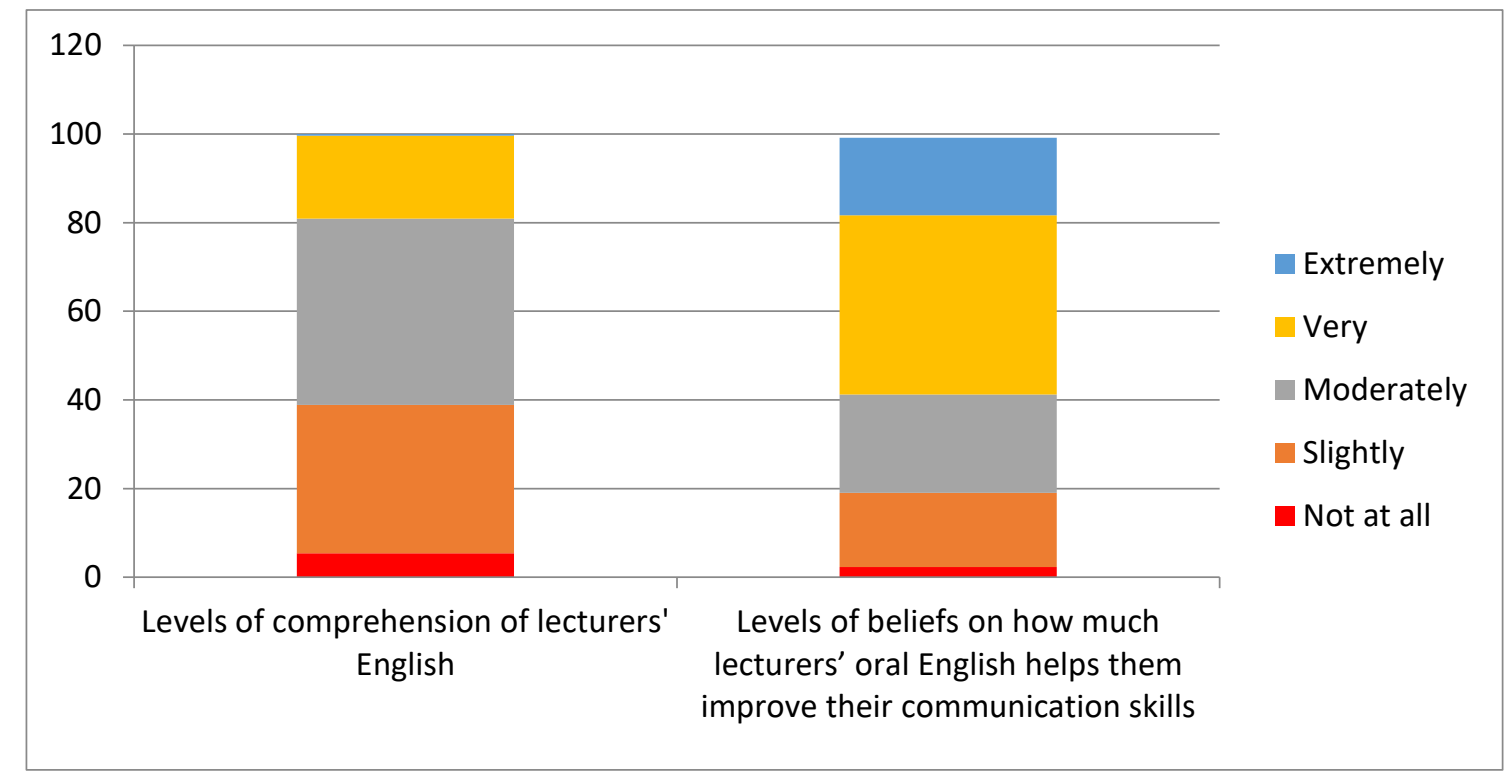

The number of students who chose Not at all and Slightly regarding the extent to which they understood lecturers' English accounted for 38.9\%, about twice as many as those labelling their comprehension level as Very and Extremely, with 19.1\%. This result gives further support to the finding from the lecturer and student interview data that students' English comprehension level was relatively low, as mentioned in 4.1.

Although a large number of students had difficulties understanding their lecturers' English, they still strongly believed that their lecturers' use of English in classroom would help them improve their communication skills. Specifically, 58.4\% students thought that lecturers' English speaking helped them improve their communication skills really and extremely, which was three times as many as those who chose the Not at all and Slightly options (19.3\%). This demonstrates that the majority of students have a positive attitude toward lecturers' classroom English speaking.

As stated in section 4.2, most of the lecturers thought that students might have negative attitudes about the lecturers using predominantly English in classes. However, findings from the above-mentioned students' perceptions of their improvement and listening motivation levels conflict with the lecturers' perceptions, to some extent. Table 4.1 summarises the results 
for Q.6 of the student survey: "Do you feel motivated, demotivated, or neutral when listening to your lecturers speaking English in class?".

\section{Table 4.1}

Student Perceptions by Motivation Levels When Listening to Lecturers Speaking English in Class

\begin{tabular}{llll}
\hline \multirow{2}{*}{ Valid } & No & $\%$ \\
\cline { 2 - 4 } & Motivated & 163 & 63.4 \\
\cline { 2 - 4 } & Demotivated & 40 & 15.6 \\
\cline { 2 - 4 } & Teutral & 47 & 18.3 \\
\hline Missing & Response & 250 & 97.3 \\
\hline Total & & 257 & 2.7 \\
\hline
\end{tabular}

There are four times as many students who said they were motivated to listen to lecturers' speaking English in class as those who were demotivated. This shows that a majority of students were motivated to listen to lecturers' English, which seems to conflict with the lecturers' perceptions that their students might be stressed and shocked when they try to use mostly English in non-English major classes.

The next section presents the factors which influenced non-English major students' comprehension of lecturers' spoken English and their learning motivation.

\subsubsection{Lecturer-Related Factors}

Speech-Performance Aspects. With regard to what actually motivated students to listen to lecturers' English, the reasons collected from student questionnaire responses were shown in the figure below: 


\section{Figure 4.3}

What Motivates Students to Listen to Lecturers' English?

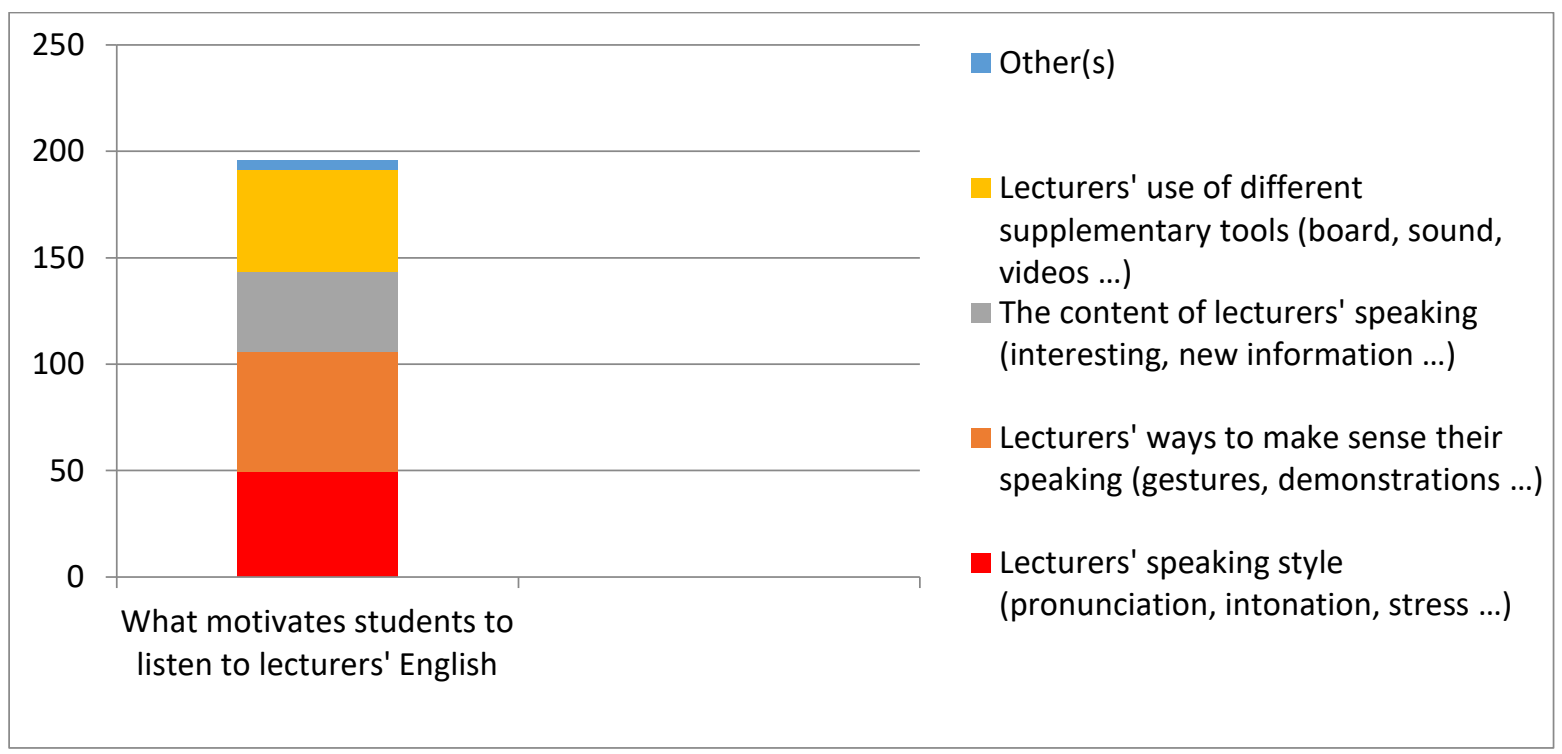

As can be seen, ways to make sense their speaking, speaking style, and the use of different supplementary tools are the three main reasons why students felt motivated to listen to their lecturers' English speaking. The content of lecturers' speech is also a motive for students to be more willing to listen to it.

In contrast, the three most common responses to the question puts them off from listening to their lecturers' use of English in class were the content of speaking, lack of eye contact and/or interaction, and speaking style, as illustrated in Figure 4.4.

\section{Figure 4.4}

What Demotivates Students to Listen to Lecturers' English?

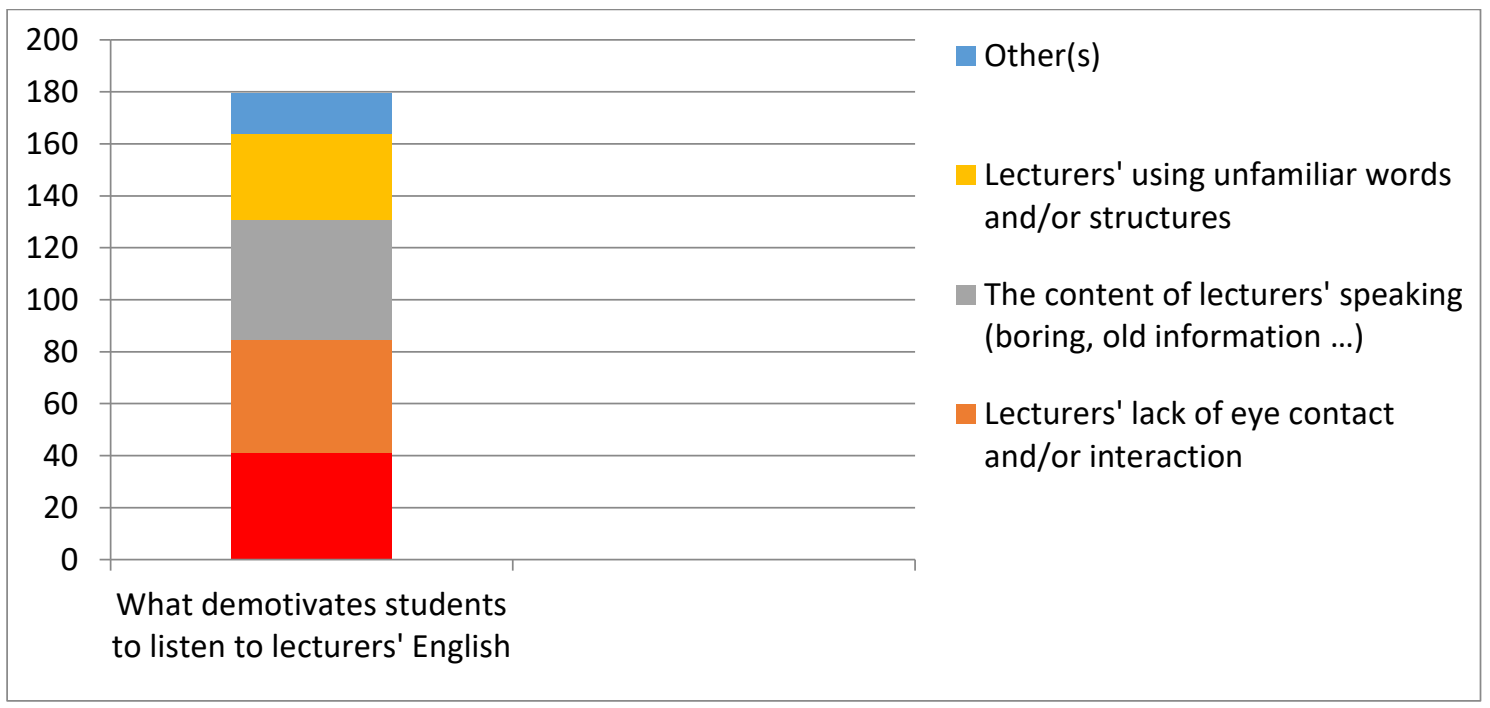


Combining the students' explanations of what motivates and demotivates them to listen to their lecturers' English speaking, we can see that aspects related to lecturers' speech performances had a strong effect on the students' willingness and motivation to listen to them. Specifically, lecturers' speaking styles, sense-making techniques, and the content of speech are found to be of concern for most of the students. Interview data further illustrate this:

I am not interested when they [lecturers] speak in normal styles. But when they speak with the up and down tones, gestures, or expressions of their faces, I am more interested and can understand some parts. (IS3)

Lecturers' English speaking is not good enough, so I do not want to listen to them. I want to listen to a native speaker or someone on youtube channels ... I think the lecturers have Vietnamese English pronunciation. (TS9)

I like studying with foreign teachers because they have good and natural pronunciation styles; They make me more confident and provide more interactive situations. (TS8)

The three students' opinions show that some lecturers' English-speaking styles were not attractive to them because it did not sound natural. Those students frequently listened to native English speakers on entertainment or social media channels. Some of the respondents also have had opportunities to study with English L1 teachers who volunteered at their college, as was the case for students from school B, or in evening classes at English centres. Therefore, they have had experiences of what native English speech sounded like and viewed the non-native accents and speaking fluency of their Vietnamese lecturers in a negative light. Research (e.g. Ellen \& Taverniers, 2011; Tergujeff, 2013) has highlighted that many students still perceive native-like pronunciation as an ideal, which is the case for the students in this study.

The survey results also revealed that $46.2 \%$ of the students found the information that lecturers conveyed in English to be boring and out-dated, which hampered their motivation to engage in listening to it. This finding shows that the topics and content of lecturers' speech play an important part in enhancing students' willingness to listen. Students further highlighted this connection between relevance of content and willingness to listen in the interviews:

The content of lecturers' speech should be relevant or related to our fields of study so that we can apply them. This would give us reasons and motivation to listen to lecturers' English speech. (TS11)

Lecturers strictly follow the curriculum and textbooks; they do not relate the content to topics we are familiar with, which would help students understand the lessons better. If so, we will pay better attention to what they are saying. (TS14)

I really like it when she [lecturer] chit-chats with us about daily topics in English; it is very friendly and natural. It feels like we are communicating in a real-life, not teaching and learning scenario; and I often concentrate better then. (IS6) 
Because the main type of motivation demonstrated by non-English major students is professional development motivation (Lobo \& Gurney, 2014), it is not surprising that they expect their English learning to link to future jobs. In other words, how the current lessons can be applied when they enter their jobs is a constant question on their mind. Therefore, they are more motivated to listen to their lecturers' English speech if it contains something that is close or relevant to their majors or potential jobs. The students in this study considered the content of lecturers' speech as a main factor that determines whether they pay attention or not. Specifically, they expected lecturers' speech to feature content or ideas that were of interest to them or that they needed for future jobs. This point was also raised by Olive, who shared that teaching non-English major students like those in her school was challenging because there were two goals: linguistic competence and professional knowledge. She also stressed that lecturers should always link teaching content to students' fields of study to ensure they maintain their interests.

According to the questionnaire results, the complexity of structures and amount and/or difficulty of vocabulary in lecturers' speech were ranked the second and third biggest barrier to students' comprehension of lecturers' English. From the five-point scale categories, the choices Somewhat strong barrier and Extreme barrier constituted $44.3 \%$ and $39.7 \%$ of all answers respectively.

The demotivating impact of complex sentence structures and unknown vocabulary were also raised in the interviews:

Lecturers should not use long, academic, or grammatically accurate sentences because those may demotivate low level students; instead, they should just use short utterances which convey the intended message or meaning. (TS4)

I think lecturers should use words which are more common and familiar in my daily life and communication. It would make me more interested in listening to them and it would be more useful because I can understand some parts of it. (IS3)

TS4 thought that lecturers did not necessarily need to use complex and academic structures because students did not need them. Short, focused, and simple phrases would make students understand them more easily. IS3 explained that the familiar and common words in lecturers' speech were necessary as they helped establish the context and provided clues for her to guess the meaning of the whole speech or at least part of it. She also believed that students would be more inclined to listen when lecturers' speech contained some words they already knew. As mentioned previously, non-English major students usually have low language competency and their motivation in learning English is practical. Hence, they normally require simple and 
common lexical and syntactic items in lecturers' English speaking which are just a bit above their zone of proximal development. During my classroom observations, I also noticed that students seemed to be more interested in short and focused utterances. The evidence presented above strongly suggests that the vocabulary and structures lecturers used in their speaking did have an influence on students' attitude towards listening to and being able to understand their speech.

Sense of Humour. Although sense of humour was detected to be only occasionally used by lecturers, the observation data show its positive effect such as making the class atmosphere more funny and interactive. The following sample situation was observed in Polly's class:

Excerpt 3: (Observation class 1 - Polly)

Lecturer: You went to the mountain. What for?

Class: (silent)

Lecturer: Played with monkey?

Class: (noisy and laughing)

Polly was teaching a speaking lesson with the topic Time expression in the past tense. She tried to ask students what they did on the previous day and there was one student saying he went to the mountain. She then continued to ask what the student did there. The class was quiet until she joked that the students played with a monkey. In Vietnamese, monkeys feature in many idioms and proverbs with different meanings. Thus, the lecturer's comment that the student played with a monkey implies that the student was crazy. Hence, the class became noisy with laughter and chatter among students. Polly's simple joke broke the silent atmosphere and created excitement.

The interview data also reveal that students appreciated their lecturers' sense of humour:

When a lecturer always smiles with students and has funny gestures or a sense of humour, students can learn more easily because it reduces their pressure. (IS6)

Lecturers should have more of a sense of humour when speaking English because we are not only bad at English listening but also have low motivation in learning. (TS12)

IS6 and TS12 thought that students would feel more motivated to learn English with a lecturer who was smiley and made jokes. They explained that, because their English competency and learning motivation was low, the use of humour by the lecturer would positively affect their learning attitude and listening motivation and make the L2 classroom setting less uncomfortable. 
That teachers' humour plays a vital role in the teaching and learning of English as a foreign language (Hidayanti, 2019) is not a new concept. Humour has been found to facilitate the acquisition of vocabulary and help distinguish figurative from literal meaning (Muñoz-Basols, 2005). Moreover, Dörnyei (2001) also commented on the use of humour by L2 teachers to create a pleasant atmosphere in the classroom, saying that teachers can use humour so that students can feel the English classroom is not threatening, especially for non-major students who have a low learning motivation and a high level of shyness. In this study, lecturers' sense of humour was perceived by students to increase their willingness to listen to their lecturers' English speech.

Ways of Providing Corrective Feedback. The interview data also show that the ways lecturers give feedback to students' errors can directly influence their learning motivation and English performances. This is exemplified by the following extracts:

And they [lecturers] should wait until we finish talking to correct our errors. Feedback to our errors is essential, but lecturers should be more patient and have more efficient techniques. Stopping me when I am talking makes me run out ideas and lack confidence to continue. (IS1)

I want my lecturers to correct phonetic and syntactic errors so that I can avoid making those mistakes again. But they should not interrupt me while I am talking; they can take notes and give corrective feedback when I finish speaking. (TS6)

Both IS1 and TS6 commented that lecturers correcting students' mistakes and errors was helpful for students' learning. This finding is supported by Lyster (2018), who proposed that providing feedback is more effective than withholding feedback in L2 instruction. In fact, most of the students in this study perceived the time and ways lecturers provide corrective feedback to them as important since they affect the students' confidence in L2 performances. The students expected their lecturers to provide feedback on their errors at the end of their speaking or at another suitable time so that the flow of their speech would not be interrupted. This would allow them to maintain their flow and keep speaking naturally and confidently in a supportive environment. Consequently, it seems that lecturers' patient and systematic ways of retrospective corrective feedback was perceived by the students to facilitate their learning.

\subsubsection{Student-Related Factors}

In addition to the above-mentioned factors, students were also aware that some barriers which influenced their motivation and comprehension of lecturers' English speaking were related to themselves. The following table synthesises the survey results: 


\section{Table 4.2}

Student-Related Barriers to Students' Comprehension of Lecturers' English Speaking

\begin{tabular}{lllll}
\hline \multicolumn{1}{c}{ Statements } & $\begin{array}{c}\text { Not a barrier at all// } \\
\text { Somewhat of a } \\
\text { barrier }\end{array}$ & $\begin{array}{c}\text { Somewhat of a } \\
\text { strong barrier/ } \\
\text { An Extreme barrier }\end{array}$ \\
\cline { 2 - 5 } No & No & 10.2 & 164 & 67 \\
\hline My lack of English vocabulary & 25 & 13.8 & 155 & 63 \\
\hline My lack of strategies in listening to English & 34 & 15.8 & 154 & 62.6 \\
\hline My lack of practicing listening to English & 39 & 16.2 & 145 & 59 \\
\hline My lack of English structures & 40 & 50.2 & 69 & 28.2 \\
\hline My lack of self confidence in listening to English & 123 & 55.7 & 48 & 19.6 \\
\hline My lack of motivation in learning English & 137 & 2 & 9 & 3.6 \\
\hline Other(s) & 5 & & & \\
\hline
\end{tabular}

Compared with the synthesised data of other factors, it was found that the students thought the root of the problem to their difficulties in comprehending lecturers' spoken English could be found within themselves. The four most commonly identified hindrances resulting from the students were: Lack of English vocabulary, strategies in listening to English, practicing listening to English, and English structures. These findings were further supported by the interview data. For example, IS7 stated: "I think my friends were honest when they say they do not know most of the vocabulary, even basic ones, because their English foundations and levels are really low". TS3 shared that the most likely obstacle was that they did not have good English backgrounds, and this was blamed on the late start of learning English in some remote highland areas. However, while most of the lecturers believed that students' low learning motivation strongly affected their willingness to practice and motivation to speak English, many students did not perceive their lack of English learning motivation as a barrier.

\subsubsection{Affective Factors}

When being asked in the survey why the students believed their lecturers' English speaking helped them improve their communication skills, the second most common reason was It feels like my lecturers really care about whether I understand or not (41.1\%). This strengthens the argument that lecturers' caring about students' comprehension ability was important in helping students improve their communication skills. The issue of how the lecturers' attitude toward students' listening comprehension affected their willingness to pay attention was also discussed in the interviews:

She speaks very quickly and does not seem to care whether we have understood her speech or not. During this time, I feel stressed and do not want to listen any further. (IS1)

It is easier to listen to lecturers and improve our skills when they check with us if we have understood them and rephrase their sentences if we have not. (TS6) 
From IS1's perspective, the speed of her lecturer's English was a challenge to her, which, combined with no comprehension check, caused her to feel stressed and diminished her listening motivation. TS6 shared that students' listening comprehension would be facilitated if lecturers cared for the students' comprehension and adjusted their speech to match the students' level. It seems that students expected lecturers to be more concerned about their comprehension. IS4 added that, if lecturers spoke English and at the same time checked how much students had understood, the students would overcome difficulties and pay better attention to them. Even the clearest instructions can be difficult to comprehend, thus, making sure that students have understood is important, especially when dealing with low level learners. Not surprisingly, the majority of students in this study expected lecturers to check students' comprehension more frequently, which they believed to be a sign of lecturers' concern and care for students. This is a feature of the affective domain which is further discussed in sections 6.2.1 and 6.2.2.

Besides expecting lecturers to care about their comprehension level of in-class knowledge, some students also thought that there was not a satisfactory connection at the interpersonal level between them and their lecturers. The students' perception of the insufficient connection between them and their lecturers aligned with the lecturers' perspectives (see 4.3.3). The students discussed this issue in the interviews:

Lecturers rarely try to know more about us such as our hobbies, preferred teaching methods or activities. I wish they would understand us more so that the lessons would be more interesting to us. (TS9)

One lecturer of mine used to ask us about our preferences in front of the whole class at the beginning of the term. But only a few students voiced their opinions. I was shy and also thought she would not have much time to answer. I think she should have conducted a simple survey so that all students could express themselves. (IS2)

The above students felt that they did not have many opportunities to present their ideas to the lecturers regarding what would work best for them. According to IS2, it seems that it was not effective to have a discussion about this topic with the whole class since students were too shy to share their ideas. This might be due to students' characteristics and the culture of the Vietnamese classroom where most students tend to be reluctant in expressing their thoughts regarding what their teacher should or should not do.

The focus group interview data also revealed an interest in a hierarchy-free relationship in classrooms among some students, which they believed would create a more relaxing and comfortable learning space. As mentioned in 2.5.3, the relationship between teachers and students in Vietnamese culture is considered particularly hierarchical and formal (Signorini et 
al., 2009). This is partially reflected in the way students stand up to greet and respond to teachers during the lessons. A lot of students in our study thought that the tradition of students' standing whenever they give responses to lecturers' questions created an asymmetrical power between lecturers and them, which hindered their learning. The following students' voices further demonstrate their perceptions:

I prefer to keep sitting when speaking English because when I stand, I will attract the eyes of my classmates and will be shy and unable to speak well. Hence, lecturers should allow and encourage all their students to keep seated during classroom communication. (TS9)

I think during English lessons, it is easy for students to exchange ideas with each other and with their lecturers when they are sitting. Having to stand up to speak means they are the focus of the other students. I am afraid of losing face should I make errors, so I think it would be better if we could speak sitting down. (IS7)

I hope that my English teachers will introduce a new rule that allows students to keep seated when replying to them. This will make our English lessons more relaxing. (TS14)

Both TS9 and IS7 expressed the view that having to stand up to speak had made them shy because they were at the centre of the attention of their classmates. Actually, they both thought that standing might be potentially face-threatening as they might make errors when speaking English. Similarly, TS14 even expected a policy set by her teachers that all students could sit speaking English. From those students' perceptions, an English classroom would be more relaxing and comfortable if the traditional power imbalance was abolished so that they could speak more freely without worrying about losing face.

Combined with the lecturers' perceptions, the above findings have highlighted that lecturers' concern about and familiarity with students, demonstrated both during classroom interaction and at an interpersonal level, symmetrical relationship, and a sense of face-saving are comfortable EFL learning space which can open up the students' learning opportunities.

\subsubsection{Contextual Factors}

Physical Layout and Classroom Interaction. According to the survey results, Lecturers' lack of eye contact and/or interaction was the second most popular reason $(43.6 \%$ responses) for students to feel demotivated to listen to their lecturers' English speaking. Lack of interaction with lecturers was perceived to be the biggest barrier (55\% responses) to students' comprehension of lecturers' English speaking in class. In the focus group interviews, some students shared their views on the current situations of classroom interaction in greater detail:

Since I started learning English at the college until now, most of the time I feel that there has been no real interaction between lecturers and students during lessons. Students hardly actively interact with lecturers and students only speak when the teacher invites them. (TS10) 
If lecturers are always enthusiastic and engaged in a two-way interaction with me, I would be more motivated. If they keep speaking by themselves, ignoring us, we will have private talks in Vietnamese and the lecturers' talk will make other friends bored as well. (TS12)

TS10 and TS12 felt that the interaction between lecturers and them was not effective enough. It appeared to them that their lecturers' speech was dominant, more of a monologue than a conversation, and students were passive listeners, which led to students' boredom when listening to the lecturers' spoken English and increased the possibility of using L1 among them. Some students expected to be more engaged in the lecturer talk by being physically closer to lecturers when they were speaking English so that "their speaking will better attract students' attentions" (TS2); and "for better hearing each other and mutual communication" (TS14). Students' engagement could also be enhanced if lecturers adopted a two-way dialogic communication style, as shared by TS12. This idea will be further discussed in section 6.2.3.

A lot of students also perceived the seat arrangement/designs and decorations of classrooms to affect their learning motivation:

The lecturer's desk should be eliminated since it creates a power distance between us; it makes it hard for those sitting in the back rows to hear her speaking at her desk. Our seats should be arranged in a U-shape so that we can all be face-to-face with the lecturer and be physically closer to him/her. The classroom atmosphere will be then more supportive; and we would feel more relaxed to study in it. (IS6)

I really like to have an individual movable seat like what [name of his friend] has said. The classroom should be decorated with pictures, posters, or slogans in English and to remind us that we are learning English. The students would be more interested and motivated to learn in such a decorated classroom. (TS11)

Most of the interviewed students agreed that the current seat arrangement and designs of their English classes negatively affected their learning motivation and hindered classroom English communication. IS6 pointed out that students in the back could not hear and see the teacher clearly when he/she was speaking at front by his/her desk. More importantly, the student felt that the seating arrangement with the teacher at his/her desk created unequal relationship in class. Hence, a more inclusive seating arrangement in class would help establish a more supportive learning environment. TS11 shared the same views, adding that the English classroom should be decorated so that students could develop a sense of belonging to English class. A classroom decorated with pictures would motivate students more than a plain one.

The above findings suggest that students perceive two-way dialogic communication, closer physical distance between them and lecturers, and an appealing classroom layout to be important to enhance their willingness to communicate and motivation to listen in EFL teaching contexts. Students' perceptions regarding the positive effect of a supportive environment for 
the success of their L2 learning aligns with the findings of previous studies (e.g. Cuellar \& Johnson-Ahorlu, 2016; Museus et al., 2017).

Assessment and Evaluation Methods. Assessment is an essential part of teaching and learning in all subjects with advantages for both students and teachers. In this study, the frequency and formats of assessment were found to have a strong influence on non-English major students' attitude toward lecturers' classroom English because the students are mostly likely to prioritise their attention to and regularly practise what was being assessed.

The lecturers involved in the study explained that students in both schools were evaluated via one compulsory mid-term and end-of-term assessment, which had two parts: one written paper taking about 40-60 minutes and one oral assessment taking about 5-10 minutes. In the interviews, students discussed their opinions about the assessment method in greater detail, as illustrated in the excerpts below:

I think there should be more small speaking tests which are done several times throughout a semester. One summative test of a few minutes has not been effective; more regular oral tests would cause students to be more interested in listening and speaking in class. (IS3)

The forms and ways of assessment are still very limited, do not measure students' ability and do not really encourage us to practice communication skills. The content/questions/topics of speaking tests is/are not interesting; oral tests should be focused on our future careers. This would motivate students to listen and practice speaking English in class and at home. (TS11)

Both IS3 and TS11 commented that only one oral assessment at the end of each term seemed to be inadequate; they desired to have more regular evaluations on speaking because assessments were driving forces for them to try to speak in English and listen to lecturers' classroom English. Moreover, the themes and topics of the oral assessments did not meet their needs and expectations; they hoped the assessment would be related and closer to their majors and future jobs. TS13 hypothesised that lecturers would automatically and naturally use more English in the class and students would have more motivation to listen and speak in English if there were more speaking assessments.

In short, an increased assessment frequency featuring more relevant content might inspire students to adopt good learning habits such as listening to lecturers' English and using more English themselves, which could increase lecturers' English-speaking motivation as well. Hence, it can be assumed that the methods of testing and evaluation could have influenced the students' attitude and motivation toward lecturers' English. This also strengthens that the Vietnamese testing approach affects teaching behaviours and practices (Kazemi \& Soleimani, 2016), and barriers to CLT (Ariatna, 2016; Rahman, 2017). 


\section{Summary}

This chapter has provided an overall picture of the lecturers' practices and perspectives on the English they used in non-English major classes along with the students' perspectives regarding their lecturers' practices.

Some key findings emerged regarding the lecturers' use of classroom English. Firstly, while both lecturers and students believed that English should be used more than Vietnamese, the observation data suggest that the lecturers actually used a slightly higher level of Vietnamese instead. As explained by most of the lecturers, this might be due to students' low comprehension level, lecturers' L2 learning experiences, and the complexity of teaching practices. Secondly, although most of the lecturers supported and realised the benefits of the English-only practice, they doubted its feasibility for the current setting and did not apply this practice because they thought that their students would not understand and subsequently provide negative feedback on the class.

Thirdly, the lecturers' accounts of what affected their English speaking fell into three categories of student-related, teacher-related, and contextual factors. The student-related factors included students' level of English proficiency, students' learning habits and motivation, and lecturers' concern and knowing about students, which were perceived to be the most influential. As for the teacher-related factors, proficiency maintenance, skills in English presentation, and personal characteristics were perceived to affect the lecturers' motivation and practices of English communication. Lesson skills/contents and class size were identified as two contextual factors.

Fourthly, the students perceived four main factors which influenced their comprehension of lecturers' English speaking and their learning motivation. The lecturer-related factors included lecturers' speaking styles, speaking content, use of vocabulary and structures, sense of humour, concern about students' understanding, and ways of providing corrective feedback. The students also perceived affective factors including lecturers' concern and understanding about them, the establishment of a symmetrical relationship, and face-saving strategies as affordances to their learning. Classroom interaction opportunities and assessment and evaluation methods were contextual elements. Lastly, students' English vocabulary, strategies in listening to English, and listening practice are student-related elements which were believed to be the most influential. 
One important aspect of the exploration of lecturers' English in the classroom that has not been discussed yet are the strategies the lecturers used when speaking English. This is the focused of the next chapter. 


\section{Chapter 5. Lecturers' English Communication Strategies}

In this chapter, findings about the lecturers' English communication strategies are presented, which helps answer research questions 2, 3, and 4. Data were analysed, synthesised, and presented from the results of 20 classroom observations, 257 student questionnaire answers, five student group discussions, and five lecturer interviews. The lecturers had at least six years of teaching experience and the students were non-English majors whose proficiency level were quite low. This chapter is broken into seven sections, and each section focuses on the findings for one of the strategies that were used by the lecturers.

\section{An Overview}

Based on the classroom observation data analysis, it was found that the lecturers in the two schools employed various types of communication strategies when speaking English to students. The different strategies and how often they were used (as given in raw frequency counts and percentages) are presented in Table 5.1 below.

\section{Table 5.1}

Frequency and Percentage of Strategies Used by Lecturers from Classroom Observation Data

\begin{tabular}{lll}
\hline Strategies & $\begin{array}{l}\text { Frequency (Averaged } \\
\text { Number of occurrences) }\end{array}$ & Percentage \\
\hline Self-repetition & 40.6 & 28.9 \\
Code-switching & 32 & 22.8 \\
Translation & 28.6 & 20.3 \\
Interactional & 18.6 & 13.2 \\
Simplification & 12 & 8.5 \\
Nonverbal & 6 & 4.3 \\
Humour & 2.8 & 2 \\
Total & 140.6 & 100 \\
\hline
\end{tabular}

Table 5.1 shows that all the lecturers used seven main strategies with different levels of occurrence. The observation data revealed humour as a new strategy, which was not included in the initial communicative taxonomy described in Chapter 2 (section 2.2.2). Humour was apparent in both verbal and nonverbal forms and was used to clarify meanings and draw students' attention to lecturers' English speaking. Based on their frequency of use, these seven observed strategies can be divided into high frequency, medium frequency, and low frequency groups.

Self-repetition, code-switching, and translation strategies fall into the most frequently used group and all three strategies presented with quite similar occurrence levels. These three strategies made up a total of $72 \%$ of all the detected strategies and were clearly the ones most 
commonly used by the lecturers. The preference for those three strategies also emerged from the interview data; some aspects of these strategies have already been discussed in sections 4.1 and 4.3. The lecturers used L2 repetition for different purposes and reasons, such as getting better attention from students or offering more L2 input for students to enhance their memorisation. Code-switching and translation were used for comprehension checking, interpretation assistance, time saving, and as habits.

Interactional and simplification strategies were used at a medium frequency, with a total of $21.7 \%$. Interactional strategies were found to be used by the lecturers to prevent communication breakdowns, facilitate the exchange of information, and increase interaction. This was achieved by the use of context markers let's see/ you know/ that's it ...; saying some words or strings of word much longer, louder, or higher or clapping hands before or after an making an utterance; summarising what they have said; and checking for student comprehension by asking questions. The lecturers used simplification strategies such as replacing simpler vocabulary/structures/messages, illustrating, or describing the properties of the target language.

The low frequency group included nonverbal strategies which made up 4.3\%, and humour occurring with the lowest rate of $2 \%$. As an English communication strategy, the lecturers' nonverbal behaviours were mainly gesturing and body language to model action verbs, phrases, and utterances to help students understand what they meant. Humour strategies were found in both verbal and nonverbal communication, which made lecturers' English speech more lively, attractive, and increased the effectiveness of communication.

Looking at the specific strategies used by individual lecturers, some similarities and differences can be noted. Table 5.2 synthesises the strategies used by the five lecturers in classes:

\section{Table 5.2}

Frequencies of Strategies Used by Lecturers from Classroom Observation Data

\begin{tabular}{|c|c|c|c|c|c|c|}
\hline Strategies & $\begin{array}{l}\text { Ellen } \\
(40.8 \\
\text { minutes })\end{array}$ & $\begin{array}{l}\text { Amy } \\
(41.8 \\
\text { minutes })\end{array}$ & $\begin{array}{l}\text { Polly } \\
(11 \\
\text { minutes })\end{array}$ & $\begin{array}{l}\text { Olive } \\
(35.5 \\
\text { minutes })\end{array}$ & $\begin{array}{l}\text { Una } \\
(32 \\
\text { minutes })\end{array}$ & $\begin{array}{l}\text { Total } \\
(161.1 \\
\text { minutes })\end{array}$ \\
\hline Self-repetition & 48 & 46 & 19 & 58 & 32 & 203 \\
\hline Code-switching & 37 & 32 & 26 & 37 & 28 & 160 \\
\hline Translation & 29 & 28 & 21 & 41 & 24 & 143 \\
\hline Interactional & 19 & 20 & 14 & 22 & 18 & 93 \\
\hline Simplification & 15 & 17 & 5 & 16 & 7 & 60 \\
\hline Nonverbal & 6 & 8 & 5 & 7 & 4 & 30 \\
\hline Нитоит & 2 & 6 & 3 & 2 & 1 & 14 \\
\hline Total & 156 & 157 & 93 & 183 & 114 & 703 \\
\hline
\end{tabular}


Table 5.2 shows that the lecturers shared a similar trend in the use of strategies, despite the rank of each strategy varying slightly between them. There are also differences in numbers of strategy occurrence amongst those lecturers, due to the varied recorded English-speaking time. However, they all made a high frequency use of self-repetition, code-switching, and translation and low use of nonverbal and humour. The remaining sections describe how each strategy was used by the lecturers.

\subsection{Self-repetition}

Teacher repetition has been regarded as an important condition for target language comprehension and performance (Jensen \& Vinther, 2003). During classroom interaction exchanges, teachers use two main discourses of repetition. The first is repeating parts of student speech for pedagogical purposes such as corrective feedback. The second is self-repetition of their own speech as a communication strategy, which is the object of this study. In this study, self-repetition as an English communication strategy ranked highest in terms of frequency. As stated in 2.2.2, self-repetition is viewed by this study simply as repeating syllables, words, phrases, or structures within one flow of speech. In the current data, repetition at word and phrase levels was found to be the most popular. All the lecturers tended to employ repetitions more often for task instructions and questions, as compared to statements or explanations.

One form of self-repetition the lecturers employed was to repeat the word or phrase that conveyed the main idea of their utterance. Followings are some sample extracts:

Excerpt 4 (Observation class 1- Ellen)

Check-up now it's time for check-up

Excerpt 5 (Observation class 1- Olive)

Now read the conversation carefully read the conversation carefully

Excerpt 6 (Observation class 1- Una)

Now who can who can help me to change two sentences into negative form? Help me please! In excerpt 4, Ellen introduced the activity check-up, which is the key noun in her utterance. She then repeated it in order to stress her instruction and call for students' listening. In excerpt 5 , Olive said the imperative phrase read the conversation carefully twice. This was because it was the main message of her speech and what she wanted students to do. Question words were also repeated, as in the case who can in excerpt 6 . In these cases, the lecturers used verbatim repetition to make their speech more focused and better draw students' attention to their utterances. This was found to be the strategy most commonly used by all the lecturers. 
Another form of self-repetition noted in the data was the repetition of new words/phrases which belonged to parts of a lesson. These were repeated to give students more exposure to them as L2 input for better memorisation. This form is illustrated in the excerpts below:

Excerpt 7 (Observation class 2 - Ellen)

Ok let's come to the first kind of (paused) ah company. Limited liability company yeah limited liability. What do you know about limited liability?

Excerpt 8 (Observation class 1 - Olive)

After starter what comes next? yes, so we call that is the main dish or main course (paused) main dish or main course

Ellen was teaching a lesson about legal forms of organisation and limited liability was a new concept for students. In the excerpt, she repeated this phrase three times to make sure students had caught its pronunciation and then started to explain its meaning. Similarly, Olive repeated main dish/main course and spoke the words much more slowly because it was new vocabulary in the lesson. The combination of slow speech and repetition might have been intended to help students memorise the pronunciation and meaning of new lexical items, which can reinforce students' memory and help students get used to listening to English (Marzuki et al., 2016) as well as support short and long-term vocabulary retention (Altalhab, 2018).

The third form of self-repetition involves the use of self-repetition to elicit and/or encourage students' responses to a question, which has been noted as a routine practice in different settings (Zemel \& Koschmann, 2011). The following excerpts illustrate two cases in which the lecturers sought students' responses to their targeted questions or follow their directions:

Excerpt 9 (Observation class 1 - Una)

Add $e d$ if they are they are (paused 3 seconds) they are (rising tone) they are regular verbs Excerpt 10 (Observation class 2 - Amy)

So why is this sentence wrong, students? (falling tone and paused) ... So why is this sentence wrong, students? (turned up tone and paused)

Una intentionally repeated they are four times, combining with pauses and rising tone to mark her utterance as a question posed to students: They are what?. After a while, she gave the answer to complete her speech flow. Una took advantage of this repetition to wait for students' responses. In the same vein, Amy repeated her question along with pausing and changing tone in order to initiate students' thinking and pursue responses from them. It was observed that, after asking the question, she changed her speaking tone and paused some seconds looking around to look for students' responses. After a while, she repeated the question and did the 
same. This shows that by this repeating, her intention was to wait and seek for responses and answers from students, as a pedagogical strategy.

Lastly, self-repetition has been considered as a self-repair strategy (Krishnan et al., 2018), or an indicator of speech disfluencies (Kovac, 2016). In this study, when the lecturers realised that an incorrect word had been used, they employed repetition in order to repair their own speech, to re-establish the fluency of the speech, due to uncontrolled mistakes in L2 production, as in the following two examples:

Excerpt 11 (Observation class 2 - Polly)

How did you feel how do you feel?

Excerpt 12 (Observation class 1 - Olive)

So I will have the word (paused 2 seconds) oh a word one by one a word on the board

Since a classroom is a unique and complex context where a lecturer has to deal with different tasks at the same time, mistakes and errors in L2 performances are inevitable and part of the natural flow of oral language, especially for English L2 speaking lecturers. This is also what some lecturers raised during the interviews: "Constantly switching between Vietnamese and English causes me to easily make mistakes when speaking English" (Una). When making mistakes, the lecturers chose repetition with adjustments to reproduce correct utterances. In excerpt 11, Polly recognised that she had misused the verb tense and repeated the utterance one more time replacing do with did to make sure the whole question was accurate. Similarly, in excerpt 12 , Olive had to repeat word because she misused the article the and immediately replaced it with $a$. Here the lecturers realised the importance of being good language models with correct grammar; hence, they did not hesitate to self-correct their mistakes and errors with the use of self-repetition. In this case, the lecturers' self-repetition appears to have both pedagogical and communicative functions. This might be because FL classroom communication contexts are different from others as lecturers seek not only to make sense with their words but also act as a language model for students (see 6.1, for more discussion).

Although self-repetition was found to be the strategy most commonly used by all the five lecturers involved in this study, only two lecturers mentioned it during the interviews and in the questionnaires. Una shared that repetition was a common strategy choice: "I often speak again and again so that students can get acquainted and understand my English speaking". Polly said that she intentionally repeated words many times so that students could listen again and again and then remember to use them. This shows that not all the lecturers were aware of their repetition use as a way to help their students better understand their spoken English. They might 
have thought it was a general teaching pedagogy or habit; or they could have used it subconsciously.

Some students were also aware of lecturers' self-repetition:

When new and difficult vocabulary or structures are used, lecturers should say it again and again; students can rely on the contexts to understand the meanings of those words and usages of those structurers. (IS5)

If students do not understand it [lecturers' English] for the first time, lecturers' repetition for five or six times combined with gestures or demonstrations will work. (TS9)

Both of IS5 and TS9 believed that lecturers' self-repetitions facilitated their comprehension of new lexical and syntactic items. Meanwhile, they thought that lecturers' self-repetition combined with contextual clues and other techniques would be effective in making them find out the Vietnamese meanings. Yet, there were some students who thought that lecturers' selfrepetitions were not always effective:

When speaking English, it is good that lecturers have repetitions, but they should be considerate of what and how many times something is repeated. Sometimes I see lecturers repeating certain words too much, which is boring for students to listen. (TS5)

Besides, I think lecturers should reduce repetition times for the structures and vocabulary that students are already familiar with. It takes time and is not necessary. (IS3)

This implies that lecturers could be more selective with their use of repetition.

In summary, lecturers' self-repetition as an English communication strategy in this study was the most common communicative strategy used in the data. Four main functions of selfrepetition were identified: stressing their speech to get better attention from students; creating more L2 exposure for students to enhance their memorisation; initiating students' thinking and pursuing their responses; and repairing their own mistakes. Despite this, not all the lecturers were well-aware of their use of self-repetition as a communication strategy. Two students thought that some of lecturers' repetitions were unnecessary and ineffective for them because they seemed to be overused.

\subsection{Code-switching}

Code-switching is one of the communication strategies used by both teachers and learners in EFL classrooms (Ayaz, 2017). The reasons behind the practice of code-switching are complex and varied (Ewert, 2010) because "it is not always performed consciously [and] as such it is regarded as an automatic and unconscious behaviour" (Modupeola, 2013, p. 93). As mentioned in 2.2.2, code-switching as a communication strategy in this study refers to the use of L1 words/chunks/turns in L2 speech for communicative purposes. From the classroom observation 
data, code-switching was found to be the second most frequently used strategy among three out of the five lecturers.

The first type of code-switching involves lecturers switching from English to Vietnamese to check students' comprehension of their English, as in excerpts 13 and 14:

Excerpt 13 (Observation class 1 - Amy)

Now everybody stand up stand up and move (students were moving) and now the mixed colour come here mixed colour (lecturers looked around the class). Rồi các em hiểu nhiệm vụ của bài tập chưa? [Have you understood what you will have to do in the task?]

Excerpt 14 (Observation class 1 - Ellen)

Lecturer: Class, do you do understand?

Students: yeah (mixed soft voice)

Lecturer: Can you explain it in Vietnamese? (paused for 5 seconds) Em nói là em hiểu cái hướng dẫn của cô đúng không? Bây giờ em nói lại bằng tiếng Việt cô nghe thử [You said that you have understood my instruction. Now please say that in Vietnamese]

After Amy instructed how students could make groups based on their clothes' colours, she asked the students to form their groups. She looked around and recognised that some students did not identify their groups. Hence, she turned to Vietnamese to check if students were following her direction. Ellen gave direction for a check-up activity in English. She then asked the class if they had understood and got different reactions, which made her resort to Vietnamese to check how much those students had understood what she meant. By switching to L1, the lecturers check students' comprehension of class content.

The second form of code-switching to Vietnamese identified from the data was as a way for lecturers to assist students' interpretation of English meanings. As illustrated by the excerpts provided below, the lecturers used code-switching to further explain or elaborate lexical or syntactic units:

Excerpt 15 (Observation class 2 - Ellen)

Now let's talk more about joint stock company. Two kinds of shares ah ordinary and ordinary ordinary. Chúng ta có động từ thường là ordinary verb go make and now we have ordinary shares nghĩa là gì? [We have ordinary verb meaning "ordinary verbs" like, go, make... and now we have ordinary shares what does it mean?]

Excerpt 16 (Observation class 1- Una)

How do you understand irregular verbs?. Các bạn chú ý lên đây cho cô chúng ta có thể hiểu như sau [class, pay attention here we can understand as follows]

In excerpt 15, Ellen switched to Vietnamese when she tried to explain different meanings of ordinary and then wanted to apply that definition to the context of economics. Una used the same strategy when she elaborated on how to use irregular verbs in the simple past tense, as 
in excerpt 16. These lecturers switched to Vietnamese because they thought it would be too complicated for students to listen to explanations in English or it would take a lot of time, as discussed in 4.3 .

The lecturers sometimes code-switched within one utterance, which was to give students L1 contexts for better decoding L2 meanings, as in:

Excerpt 17 (Observation class 2 - Polly)

Có two tourist destinations ở Quảng Ngãi [There are two tourist destinations in Quang Ngai] Excerpt 18 (Observation class 1 - Ellen)

Raise your hand nếu em có câu trả lời! [Raise your hand if you have the answer)

Polly switched to L1 phrases which were quite simple and kept the L2 phrase that was much more difficult, which stands in contrast to normal cases when teachers resort to L1 when its L2 equivalents are abstract or difficult for students to understand. This might be because she would like to stress L2 words and use L1 as context cues for students to allow them to gauge the meaning of L2 more easily. A similar situation is found in excerpt 18 when Ellen combined L1 and L2 in the same sentence. Here the L1 phrase gives contexts and clues which facilitate the decoding of the L2 phrase. In the two cases, code-switching was used to scaffold the learning of L2, providing a bridge from what is known and unknown, especially for difficult concepts (Vu \& Burns, 2014). The lecturers gave necessary contexts and clues for students in L1 so that they could find out the L2 meaning of tourist destinations and raise your hand. This is called contextual support - one of the six scaffolding principles (Van Lier, 1996). Code-switching to L1 as a scaffolding strategy was also noted by Antón and DiCamilla (1998) who argued that the L1 allows learners to scaffold new knowledge, mediates their cognition, and evaluates their understanding of a text in an L2. In these cases, the lecturers' L1 functions as a scaffolding mechanism, a method to build on existing knowledge, which facilitates learners' L2 learning (Bhooth et al., 2014).

Thirdly, the lecturers were also found to switch to Vietnamese instead of continuing with English for classroom management or affective purposes. This type of use is illustrated by the following examples:

Excerpt 19 (Observation class 1 - Amy)

So group 2 is the best group for this activity. So tell me your name, Truong, Ngoc, Hung, sorry Truc, Ngoc, Hung, Nghia. Ngọc cho cô biết là trong nhóm tất cả các bạn có xứng đáng được điểm tốt không nè? [Ngoc, do all the members in your group deserve good marks?]

Excerpt 20 (Observation class 1 - Una) 
Finished exercise 1? Vở bài tập đâu rồi? các bạn có muốn học không? [Where are your notebooks? Do you want to study?]

Excerpt 21 (Observation class 2 - Ellen)

Yes. Remember what's his name? Bình thường chắc không đến nỗi khó phải không các em? Giờ áp lực quá nói không có ra [Normally it may be not that difficult for you, right? Now you are too stressed to make correct sentences]

After summarising the group work and asking for names of students in the group which scored the highest, Amy used Vietnamese to ask the group leader if all the members deserved good marks for their contribution. Similarly, Una was displeased because some students did not do an exercise they were asked to do. After using English for the initial question if they had completed the task, she admonished them by asking questions in Vietnamese. In those two situations, the lecturers used L1 to manage class and student behaviours. Ellen gave encouraging words to students after they made a lot of unusual mistakes in their answers to a simple question, which was a big surprise to her. Ellen's switching to L1 can be said to be related to affective purposes because she wanted to encourage and comfort students after their inaccurate response.

The fourth code-switching strategy found in the data involves the lecturers' code-switching when they changed the topics/themes of the speech. This was mostly used for out-of-lesson communication, as the cases of Polly and Ellen below demonstrate:

Excerpt 22 (Observation class 1 - Polly)

It [Quang Ngai city] is famous for garlic. Thien An pagoda. Các em có muốn nghe lịch sử chùa Thiên Ân không? Ngôi chùa này ... (continued with Vietnamese) [Do you want to know some myths about Thien An pagoda? This pagoda ....]

Excerpt 23 (Observation class 1 - Ellen)

I will say, I will say some words and you will finish the sentences, you make the sentence with those words, telling the truth. Nhớ đừng nói là cô giáo tiếng Anh của em 25 tuổi hay em có một đứa con trai là được hì. Nó không có đúng! [Remember, do not say "my English teacher is 25 years old"; or "I have a son". They are not true!]

When a student mentioned visiting Quang Ngai city, Polly added that this city was famous for the Thien An pagoda. She then used Vietnamese to share some myths about this pagoda, which was not a part of the lesson content. Ellen required students to make true sentences beginning with the words she gave. She then resorted to Vietnamese, joking about examples that would be untrue. It seems that the lecturers used this type of code-switching to create a more relaxing and interesting classroom atmosphere.

The last code-switching type accounts for cases where lecturers switched to Vietnamese to save time. This used is exemplified in the following excerpts: 
Excerpt 24 (Observation class 1 - Amy)

Now everybody. Stand up! Từ từ cho cô kết quả của bài hồi nãy nè nhóm nào làm tốt nhất nè

[Wait! Please give the results of the latest activity. Which group did the best?]

Excerpt 25 (Observation class 1 - Olive)

Exercise number one two it is very easy now one two. Làm nhanh bài tập số một, hai. Năm phút thôi xong bài này [Do exercise one and two. You have only five minutes to finish it]

It was not until Amy gave instruction for the new task that she remembered she had not evaluated or given good marks for the best group for the previous activity. Once she realised her mistake, she switched to Vietnamese to explain the situation to the class more quickly. Olive used Vietnamese to urge students to do the task quickly and reminded them of the time limit. She did not include these in her English instruction because it was almost the end of the lesson. I also noticed the faster speed in their Vietnamese speech in those two situations, which indicated that code-switching was the lecturers' choice when they were under time pressure. This is also supported by the lecturers' accounts in the interviews. For example, when talking about the choice between English and Vietnamese, Una said: "The lesson plans require me to be very fast; so for situations which are not related to the lesson contents or are unnecessary, I use Vietnamese".

To sum up, this study found five main functions of the lecturers' code-switching to Vietnamese; they are: checking for L2 comprehension; assisting L2 meaning interpretation (giving further explanations/elaborations and scaffolding); talking about out-of-lesson topics/situations; saving time; and for classroom management/affective purposes.

\subsection{Translation}

The lecturers' English communication strategy translation is defined in this study as translating literally any L2 lexical and syntactic units into L1. Translation was the third most frequently used strategy in the data, where it was found to serve several functions. These different functions are analysed and described below.

Firstly, the lecturers translated English words or phrases into Vietnamese to ensure students' English comprehension. This was the most commonly used function of translation in the data.

Excerpt 26 (Observation class 2 - Amy)

What is it function? Function là chức năng đó các em. ["Function" means duty, dear] What is it for?

Excerpt 27 (Observation class 2 - Una)

Wow (laughed) ok. When do you usually go on vacation now answer my question when do you usually go on vacation? Vacation it's your idea. Vacation là kì nghỉ đó các em ["Vacation" means holiday, dear] 
As can be seen in the data above, the lecturers only translated those words/phrases in their utterances which were new or contained the key concepts. In the above extracts, Amy and Una used English to ask the class a question and translated function and vacation to Vietnamese because they were key words in the lessons. Translation of this type was often found to occur with repetition (as the case of Una) or simplification strategy (as the case of Amy) to clarify the meanings of lexical units.

Similar to code-switching, the lecturers also translated content to give full explanations and elaborations for grammatical rules or lesson contents. In these cases, the lecturers did not only provide a full translation but expanded on the topic and gave more detailed explanations in L1. This function is illustrated in the following extracts:

Excerpt 28 (Observation class 2 - Olive)

I don't love you at all! So in the negative way "at all" in the negative sentence. Tôi không yêu bạn tí nào cả. Vậy ở dạng phủ định người ta dùng "at all" ở cuổi kèm với "not" để nhấn mạnh tính phủ định của câu. Ví dụ như ... ["I don’t love you at all". So in the negative form beside "not", we use "at all" at the end of the sentence to stress the negative mood. For example, ...]

Excerpt 29 (Observation class 1 - Amy)

So it (the main idea) is about the definitions and different types of operating systems. Ý chính của bài này là định nghĩa và các loại hệ điều hành khác nhau. Các bạn học chuyên ngành khoa học máy tính đã biết hệ điều hành rồi phải không? [the main ideas are definitions and different types of operating systems. You are majoring in computer science, so you know operation system, right?]

In excerpt 28, Olive explained the meaning of the linguistic unit not ... at all. She translated all the utterances into Vietnamese and elaborated on its functions with further examples. Amy wanted her students to have a full understanding of the main ideas of the reading passage, which was the overall goal of the lesson, by providing a Vietnamese translation. She also emphasised that those students might have known what the operation system meant, as shown in excerpt 29.

As well as word-by-word and sentence-by-sentence translation, the lecturers also translated only key points/ideas after full instructions or explanations in Vietnamese. For example, the following extract shows how Amy explained the rule of the activity in L2, followed by an L1 translation for the salient points only:

Excerpt 30 (Observation class 1 - Amy)

Now open your book and tell me (paused) skim the text and tell me what is it about as quickly as possible. The main ideas of the text. Two good marks for the correct answers for the first for the quickest ones. Đọc và tìm ý chính cho đúng để kiếm hai điểm tốt nhé! [Read and find the main ideas to get two good marks, dear!] 
As can be seen, Amy first gave a long list of directions in English, which was then cut down to read and find main ideas to get two good marks in Vietnamese. These were the key message what she wanted students to focus on. This function was often used after the lecturers gave long instructions, explanations, or statements. Its use may have been motivated by the lecturers wanting to make sure that students had understood what was meant.

At the same time, based on their tone of voice, facial expressions, and the use of tag-word affective markers nào or nhé (which mean dear) at the end of each Vietnamese translation in extract 30, it seems that the lecturers' use of translation was also related to affective functions (Bruen \& Kelly, 2014; Caldwell-Harris, 2014). Thus, the lecturers seemed to want to reassure students, reduce their anxiety, and encourage their engagement/response when using translation to L1. This affective function is called interpersonal relation because lecturers would like to build/strengthen interpersonal relationships with students or to reduce students' anxiety (Leoanak \& Amalo, 2018).

The first two mentioned functions were also described by some lecturers in the interview, as illustrated in the extracts below:

When I was speaking English and students seemed to be unhappy and begged me to speak Vietnamese, I then used translation to make them happy and understand the lesson better. (Amy)

In first-year classes, I usually speak English, followed by Vietnamese translation. This helps students better understand my English and reduces their fear and shock; they have just transitioned from a high school English learning environment. (Una)

Amy commented that she based the decision to use translation on students' reactions to her English speaking. She believed that the use of translation would not only enhance students' comprehension but also make students more comfortable and less stressed. In the same vein, Una thought that she used more translation for classes of year one, because those students had just transitioned from the high school English education environment where they had not been exposed to a lot of English. This shows that Una wanted to give her students time to adapt to the dominant use of English in their classes. Amy's and Una's decision on their use of translation is closely linked to their concern for students' learning experience. Also, they believed that translation into English was a useful way for students to improve their comprehension of English.

Finally, it seemed that translation was not always intentionally used by lecturers but was a kind of habit. From what was recorded from the lecturers' speech, we can see that they often translated common simple classroom phrases such as "Thank youl Who elsel Come back to 
your seat/ Keep silent/ Do you understand?/ Listen again/ One more" into Vietnamese. Moreover, the translations of those phrases were not consistent throughout the lessons. For example, within one lesson, Una, Amy, and Polly did not translate "Do you understand?" when it occurred the first times but some other times they did. The lecturers' unconscious use of translation was also noted in the student interview data. As shown in the extracts below, some students described translation to L1 as also part of their lecturers' habit:

I also do not like the teachers who are always translating into Vietnamese, but it seems that they are accustomed to it (translation to Vietnamese). (TS1)

Like habits, some teachers often translate to Vietnamese, even for simple English phrases that we already know. (IS2)

TS1 and IS2 were aware of their lecturers' use of translation. Since their lecturers would translate simple phrases which the students already knew, they suspected that the lecturers sometimes translated things out of habit.

When asked in the questionnaire what helped them when they did not understand their lecturers' English speaking, most students (116 out of 253, taking up 45.8\%) selected "The combination of demonstrations and translation into Vietnamese". The second most popular choice was "Translation into Vietnamese with explanation of new words/structures" with 29.2\%. This survey data show that students thought lecturers' L1 translation was helpful, but it seemed that this was not enough for them. They expected their lecturers to combine translation with other ways such as demonstrations or explanations. Students further elaborated on their perspectives on lecturers' use of translation in the interviews:

It is true that a lot of students cannot understand lecturers' English; but lecturers' using too much translating is not effective to help us improve. (IS4)

It is not necessary for lecturers to constantly translate every single sentence into Vietnamese; we have the ability to figure out the meaning. If we cannot get the message right away, listening to it three or four times definitely helps. (TS7)

Lecturers should use translation after they have tried other ways unsuccessfully. (TS6)

To help students understand their English speech, I reckon that lecturers can give cues or synonyms, instead of translation; they should only translate into Vietnamese when other techniques do not work. (TS13)

IS4's and TS7's ideas both suggested that lecturers overused translation, which did not help them improve their English. TS6 and TS13 seemed not to be in favour of their lecturers' use of translation as a way to enhance their comprehension because they thought there were other more effective ways. When students did not understand lecturers' English speaking, they thought that lecturers' translation was only helpful when it was combined with other 
techniques. In other words, not all lecturers' translations were effective and necessary for students. Translation, according to the students, should be the final option following other ways such as demonstrations, simple explanations, cues, or synonyms.

In conclusion, there are three main functions of the lecturers' use of translation to L1 in this study. Firstly, they would like to enhance students' comprehension of L2 vocabulary, structures, or messages. Secondly, translation also served an affective purpose as the lecturers took advantage of translation to Vietnamese to show they concerned about the students. Lastly, translation to L1 was a part of the lecturers' teaching and speaking habit. Some students thought that lecturers' L1 translation was sometimes too much and did not help them improve their communicative skills. Others believed that translation was helpful as a final step after lecturers tried other techniques such as exemplifications or giving synonyms/cues.

\subsection{Interactional Strategies}

In this study, the term 'interactional strategy' refers to the use of techniques such as comprehension check or summary that serve to keep the communication channel open. These interactional strategies serve to give the listener time to decode the meaning and enhance students' attention during the interaction. Interactional strategies were found to belong to the medium-use group. Nonverbal strategies were not included here, as explained in section 2.2.2. The most common interactional strategies used by the lecturers were fillers, as exemplified in the following excerpts:

Excerpt 31 (Observation class 1 - Amy)

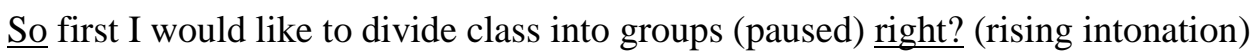

Excerpt 32 (Observation class 1 - Ellen)

I will read a letter. I will read a letter ha and you will write the word yeah (paused) beginning with that letter and the word relates to the topic well family and friend, okay?

Excerpt 33 (Observation class 2 - Polly)

Okay how was the weather? Yeah did you have nice weather?

Amy inserted "so" at the beginning of the utterance to transit and mark a new utterance. Discourse markers were also used at the end of each speech such as "right" and "okay". This was often combined with pauses or rising intonation to check if students had heard or comprehended what the lecturer had said. In excerpt 32, Ellen was giving a long instruction and used three fillers to allow time for students to decode parts of the instruction. The fillers were put right after key nouns such as letter, word, and before family and friend which served to stress the key message. After asking the question, Polly used the filler yeah to keep the 
communication channel open while waiting for answers from students, as in excerpt 33 . Those discourse markers could also help establish classroom interpersonal relationships and create a better atmosphere for learners to be more engaged in communication (Chapetón Castro, 2009). They could have facilitated the process of L2 meaning interpretation in spoken interaction and ensure that interactions run smoothly (Lam, 2010).

Apart from fillers and discourse markers, the lecturers also used emphasis of key words and phrases in their speech during interactions to draw students' attention. There were several ways of doing this. The lecturers emphasised key words (in bold) by saying them very loudly, such as tried in Olive's utterance: "What have you tried (said loudly)? Tell me please what have you tried (said loudly)?" (Class 1). Another way to add stress was to rise intonation, as in Una's speech: "How many kinds of questions do you have (rising intonation)? kind (rising intonation)? (Class 1). Saying some words much more slowly than others was another way to emphasise them, as in: "So Sam is a boy a male voice (said slowly) we hear voice we refer to a male voice (said slowly)" (Class 2 ). The lecturers intentionally and unintentionally changed the ways they spoke to get students' attention, and to convey meaning. Getting students' attention is an essential part of oral interaction because attention is the process which "encodes language input, keeps it active in working and short-term memory, and retrieves it from longterm memory" (Mackey, 2005, p. 631).

Interactional strategies also manifested in the ways some lecturers checked students' comprehension, as in the two following examples:

Excerpt 34 (Observation class 2 - Polly)

And now you work in pair work in pair. How many how many people? (paused) two, right? Excerpt 35 (Observation class 2 - Ellen)

The same number the same number of members in each line. The same number of members. What do I mean?

After assigning the type of class activity, Polly posed a question to explicitly check if her students had understood the meaning of pair. Ellen asked students to describe briefly in Vietnamese what they had to do as a way to make sure they had understood what she meant. These checking questions were common, especially after the lecturers gave instructions in English for activities or explanations for grammatical concepts.

Interactional strategies were also apparent in some of the lecturers' provision of L2 summaries to make sure students had understood correctly:

Excerpt 36 (Observation class 1 - Una) 
Ok you have five minutes to do that exercise and after that go to the board and write down your answers please very easy. Five minutes and write answer.

Excerpt 37 (Observation class 2 - Amy)

You are going to listen to two conversations, two conversations, right? We have two conversations. And you have to decide whether the sentences are True or False ah the sentences 1-3 is for conversation 1 and the sentences 4-6 is for conversation 2, right? remember (paused 5 seconds to prepare the audio) right? remember conversation 1 questions 1,2 and 3 conversation 2 questions 4,5 and 6 listen and decide true or false

In the two excerpts above, after giving task instructions, Una and Amy summarised their instructions with important words which conveyed the main message of their speech. As can be seen, the language in the summary was short and condensed. Restating key message/content gave students another opportunity to receive L2 input in a more condensed form. The use of summarising as a communication strategy could have made the L2 input more salient. This might have influenced the students' noticing the use of this strategy, which, at the extent of awareness, is a necessary prerequisite and an important tool in language learning (Schmidt, 2001).

Lastly, during interacting with students, the lecturers sometimes asked students to repeat or clarify the information given:

Excerpt 38 (Observation class 1 - Olive)

Student A: He ordered broccoli

Lecturer: Can you say again, broccoli?

Student A: broccoli

Excerpt 39 (Observation class 1 - Amy)

Student B: It's operating system

Lecturer: What is the meaning of operating system?

Student B: là cơ quan điều hành máy tính cô [It is what operates a computer, teacher]

In excerpt 38, Olive asked a student for the repetition of the word broccoli to correct their pronunciation. In this instance, form-focused feedback was given once with a phonological correction. In excerpt 39, Amy optimised the communication strategy of asking for clarification by asking the student to explain the lexical meaning of operating system, which led to more lecturer-student interaction.

Some techniques relating to the interactional strategies which were mentioned above are reminiscent of parentese, the speech produced by an adult with a young child still learning his/her first language or a more mature child when interacting with a child whose linguistic competence is perceived as limited, which is similar to the situation of English lecturers 
communicating with non-English major students. When communicating with young children in L1, adults "use short clauses, reduce the speed rate, simplify the sentences, vary their pitch more often, and broaden the range of the prosody" (Ferreira et al., 2016, p. 985). Those techniques aim at making the speech more focused and draw children's attention, which makes it easier for the children to better understand the language. Lecturers' utility of features of parentese when communicating with students can facilitate their English comprehension and acquisition.

During the interviews, two lecturers said they used interactional strategies as a way to enhance students' English comprehension:

To ensure students' comprehension, I also regularly check by asking questions or requiring one student to do a sampling. (Amy)

I normally check by asking questions. For example, I use yes - no questions to ask students what they have to do and if they have understood me or not; I also try to speak in an interesting way and I emphasise many words to maintain students' attention. (Una)

Although they did not explicitly mention any words related to interaction, Amy's and Una's accounts revealed their use of interactional strategies in the form of comprehension check and attention-getting. Two of these techniques were found to be the most commonly used among the interaction strategies.

Meanwhile, as mentioned in 4.4, most students perceived lecturers' concern about their understanding to be important to improve their communication skills and affect their comprehension. Some students also voiced their expectation for lecturers' use of interactional techniques:

Besides, I think, if lecturers speak with rising and falling intonation, and know if students have understood or not, students are more likely to be interested and want to listen. (TS12)

I have studied here for two semesters now, in the same class but with two different lecturers. This year, I notice that the lecturer has a flat intonation when speaking, no word stress, so I am not interested in listening. Last year, my lecturer was different; she spoke slowly enough, always asked if we could follow her, and put stress on certain key words, which helped keep our attention and it was easy to listen to her. (TS4)

It seemed that lecturers' speaking with emphasis and flexible intonation was believed to attract students' listening, as shared by TS12 and TS4. Those students regarded lecturers' constant check for their comprehension to be necessary and effective because it could facilitate their comprehension and enhance their willingness to listen. This demonstrates that the students had a positive attitude toward lecturers' use of interactional strategies. 
To summarise, interactional strategies were used by the lecturers for five major purposes: helping students keep better track of their spoken English; checking for students' comprehension; reinforcing L2 input for students (by summarising or expanding time); increasing attention/noticing from students; and encourage learners' participation. In fact, it is obvious that the above techniques aimed at inviting students' responses; and drew them in to the lecturers' speech. Together, they contributed to promoting lecturer-student interaction and to supporting students' L2 acquisition. Interactional strategies in lecturers' speech could also have increased classroom interaction (Putri, 2015), which could result in a more active classroom atmosphere and informal teacher-student relationship, which in turn could promote student talk. In fact, this is further supported by both the lecturers' and students' interview data where they commented on the important roles of interactional strategies and the benefits they brought to students' listening comprehension and motivation.

\subsection{Simplification}

In this research, simplification as an English communication strategy is defined as the techniques used by lecturers to simplify and make English speech better understood by students, such as substituting, exemplifying, illustrating, or describing the meaning of the original message or unfamiliar lexical units. A full description of what is categorised as a simplification strategy is found in 2.2.2. The simplification strategy belongs to the mediumuse group with regard to frequency and was applied in both spoken and written forms.

The most common way the lecturers simplified their speech was by using alternative lexical items, such as superordinate or related terms which share semantic features with the target words or structures. Some examples of this strategy are presented below:

Excerpt 40 (Observation class 2 - Una)

We will listen about Jack and Liu's vacation, right? (rising intonation and pause) their holiday. Excerpt 41 (Observation class 1 - Amy)

Now let's think about the CPU [right?] CPU how many components does the CPU have? (paused 3 seconds) how many parts are there in the CPU?

Una introduced a listening activity, which contained the new word vacation. Hence, after realising that the word vacation was challenging to students, she replaced it with holiday, another common noun, which was much more familiar to them. Similarly, Amy initially used the technical word component, which occurred in the reading text. She then substituted component for part so that students would better understand her utterance because she knew that part was more familiar to the students. As discussed in the literature chapter, using 
alternative lexical items, to some extent, coincided with the approximation strategy as proposed by linguists such as Dörnyei and Scott (1997).

To make their L2 speech easier and more accessible for students, the lecturers also illustrated or described the properties of the target object to explain the meaning of a new or strange term, as in: "I want you to talk more about routine. Now talk about yourself talk about your routine, talk about what you do every day, something that happens regularly, a routine" (Class 1 Ellen). In this situation, Ellen described the lexical properties of routine, by using lexical units that were simpler in order to support students' deduction of its meaning. One interesting point here is that she illustrated the meaning of the word twice using two different phrases to increase students' comprehension. Olive used the same approach when she wanted to help her students understand the new word tractor: "He can drive a tractor tractor. What does this mean? This is a machine for farmer a machine for farmer ... this is for farmers who work on the gardening" (Class 2). In those two cases, the lecturers used a complete sentence to replace a new complex word. They also added information to provide contextual background to these linguistic items (Chaudron, 1983). From the linguistic perspective of communication strategies, these strategies are called circumlocution or paraphrase (Dörnyei \& Scott, 1997) (see section 2.2.2).

The lecturers simplified not only words, but also complete sentences which were replaced with other ones which shared the same or similar meaning. This approach can be seen in Olive's speech: "Are the dishes familiar to you? (paused 4 seconds) Do you know the dishes?" (Class 1). In this case, only the key content noun dishes was kept and the lecturer chose the phrase do you know to replace with the adjective familiar to because she believed this was more comprehensible to students. In other words, the lecturer replaced the original message with a new one after she realised it was problematic to students' understanding. This was not found to be used as often as the two previous techniques. This phenomenon aligns with the meaning replacement category in the CSs model of Kasper and Færch (1983) and Willems (1987); and the message replacement category of Dörnyei and Scott's (1997) classification.

As can be seen, instead of using simple words right from the beginning, the lecturers chose to introduce the new term first and then simplified them to facilitate students' decoding of the meaning. Apart from word or message replacement, giving examples and sub-categories were other ways of speech simplification.

Excerpt 42 (Observation Class 1 - Ellen)

So, at first, at first, Vietnam Airline is a state company ... do you know "state"? our college our college is a state college 
Excerpt 43 (Observation class 1 - Olive)

Lecturer: If you eat ice cream what ... flavour of ice cream do you like?

Students: (silent)

Lecturer: Pardon? Do you like strawberry, vanilla, chocolate, apple, or whatever?

In order to help students better comprehend what state meant, Ellen gave an example sentence which contained state and featured contextual information that was relevant to the students. Some lecturers also used examples of sub-categories to explain the big category. This approach can be seen in Olive's excerpt, where she offers strawberry, vanilla, chocolate, and apple as sub-categories for flavour to help students find out its meaning. Providing examples of subcategories was found to be not used as often as others.

Besides using sounds and words, the analysed data also contained instances of written simplification, which was not mentioned in the communicative strategy literature. In an L2 communication context, even when students are able to understand the pronunciation of new linguistic items, it can be challenging for them to imagine how those items are spelt. To deal with this, the lecturers sometimes wrote key words, phrases, or the whole L2 utterance(s) on the board, followed by a more detailed explanation. In addition, the lecturers sometimes explained the meanings of concepts by drawing symbols or pictures on the board. For instance, after Amy said: "Now you help me mark these words. Mark it" (Class 1), she drew a tick which was the simple symbol for students to understand the meaning of mark. The use of writing or visuals helped students understand the lecturer's English speaking more easily.

Some of those simplification techniques (e.g. synonyms, flowcharts, symbols) are deemed to be mediation tools which help lead students to higher thinking (Kozulin, 2003) and provide a way for students to plan and rationalise consciously (Lantolf \& Thorne, 2007). These visualisations, examples, and models are provided by the lecturers to stimulate the students to perform tasks or enhance the message comprehension (Poehner \& Infante, 2015). In these cases, the mediation is delivered in the forms of language and symbols, which provides guidance and assistance to L2 learners (Thoms, 2012).

During the interviews, three lecturers said that they tried to use simple language to make sure that students could fully comprehend their English speech. This reflects that most of the lecturers were aware of the necessity to make their English speaking simpler so that it would be more accessible to students. However, they did not share any more insights into how they made their speech simpler, and the observation data did not feature many uses of simplification strategies either. These may illustrate that not all the lecturers were aware that simplification 
techniques could be used as communication strategies, although it was found to be used by all of them (see section 6.1, for discussion of lecturers' awareness of communication strategies). Ellen was the only lecturer who described her use of simplification strategies:

As for low level classes, I usually use simple words and structures; I also include a lot of examples, explanations, and illustrations to make it easier for them to follow and understand my English speaking. (Ellen)

To enhance students' L2 comprehension, Ellen used examples, explanations, and illustrations, which are types of simplification strategies.

According to the survey data, students indicated that explaining by speech, drawing pictures, and demonstrations were the strategies lecturers used that were most helpful to their understanding of the lecturers' English speaking. This is demonstrated in TS10's idea:

Teachers need to use really simple English and structures and we need them to have different ways to make their speech easy and simple enough for us to follow. (TS10)

In summary, simplification strategies were widely used: both for lexical and syntactic structures and both for spoken and written discourses. There were three common ways the lecturers simplified their English speech: substituting the original words/phrases/messages with new ones using synonyms or sub-categories; elaborating lexical/linguistic items by describing, illustrating, or giving examples so that they were more familiar to students; and using writing demonstrations so that students could better follow and remember the new information. Knowing that students would have difficulties comprehending their English speaking, the lecturers tried different ways to make their L2 speech simpler and more accessible to the students, which enhanced L2 learning. This kind of scaffolded linguistic support (Shay, 2006) received from lecturers could have helped learners internalise the knowledge and expanded the learners' conceptual potential (Lantolf et al., 2014). While most of the lecturers were aware of the necessity to make their English speaking simpler, only one lecturer explicitly showed their awareness of using simplification as a communication strategy. Meanwhile, most of the students believed that lecturers' simplification techniques were helpful to their comprehension.

\subsection{Nonverbal Strategies}

Because nonverbal channels can help learners both understand the foreign language and express themselves, nonverbal behaviours are also a relevant communicative strategy (Surkamp, 2014). As mentioned in section 2.2.2, by categorising nonverbal as a communication strategy in this study, I limit it to those nonverbal devices which only aim at helping make 
sense or clarifying lexical/structural units in L2 verbal speech and are excluded from interactional and simplification strategies. This means that not all nonverbal behaviours are categorised as a nonverbal communication strategy in this study. It may be surprising to see that nonverbal strategies belong to the low frequency strategy group. Although the overall occurrence level of nonverbal behaviours such as facial expressions or eye contact during lecturers' teaching could have been higher, nonverbal communication strategies - as defined above - occurred less often.

As an English communication strategy in this study, hand movements and gestures were the most commonly-used nonverbal communication strategies in the data. Most of the lecturers used their hands to point at items or enact action words that featured in their speech. For example, Ellen pointed at the desks when asking students to come back their seats or drew lines with an imaginary pen in the air to represent the action write. Olive showed her four fingers when she asked the students to pay attention to number 4 . Polly moved her hand up and down to signal when asking students to stand up or sit down. Una slapped her hand on the table when she said time is up or finish to emphasise her directive meaning because slapping hands normally conveys meaning of start or finish in the Vietnamese culture. Amy touched her ear and gestured when she asked one student to speak louder so that it would be easier for the student to understand the meaning of louder. In using those nonverbal devices as a way of scaffolding and mediation, the lecturers sought to facilitate students' comprehension of L2. In these moments, gestures and speech co-expressed the meanings, creating a shared symbolic and mental space between learners and teachers (McNeill, 1994).

Apart from being used to clarify L2 speech, the use of nonverbal strategies was found to have several other purposes. For instance, Amy sometimes snapped her fingers before some important classroom events such as checking the group work and giving good points to students. Here Amy's nonverbal behaviour seemed to mark the upcoming events; thus, it did imply a certain message to students: Attention, please!. Similarly, Una clapped her hands after she gave instructions or commands for students. From what I saw, these techniques did make some students who were focused on their tasks shift their attention back to the lecturers. They here functioned as supplementary tools to help the lecturers make their speech more compelling and to invite students' engagement in listening. The above findings are supported by Sözer's claim (2019) that body language plays an important role in drawing students' attention.

Two lecturers, Ellen and Una, said that they used a lot of nonverbal strategies when speaking English. Their comments are presented in the excerpts below: 
To create a pleasant class atmosphere and make students interested in learning, I often use gestures, repetitions, stresses, and facial expressions. These are my work routines and change over time in a way that I am often not aware of. (Una)

When speaking English to students, I use a lot of gestures, even facial expressions, or mouth and hand models. I think they are useful for students' comprehension. (Amy)

Based on Una's and Amy's perspectives, the use of nonverbal strategies was useful for students' comprehension and made students interested in their learning. Una also added that her use of nonverbal strategies was so much part of her teaching routine and she now uses them subconsciously. From a sociocultural perspective, it can be argued that nonverbal strategy use is an important sociocultural artefact and a vital dimension of human interaction in the classroom context. It has been shown to function as an important semantic resource in L2 teaching and learning contexts (Smotrova \& Lantolf, 2013). Accordingly, by using appropriate nonverbal behaviours, lecturers can scaffold students' comprehension of their English speech (students' input) and in doing this, can foster students' learning and deepen their understanding.

While three lecturers chose nonverbal strategy to be easiest to use in the questionnaire, it was observed to be in the least frequently-used group. It seems possible that this inconsistency was due to the lecturers' personalities and characteristics, which hindered them from using a lot of nonverbal means when speaking English to students, as suggested by Olive: "Actually I do not know how to explain why I did not use nonverbal much; but I think it is probably because of my personality traits". From what I observed, she was quite disciplined and a bit reserved when communicating with students in class. This might be partially affected by her position as head of the English Faculty, which may have resulted in her feeling pressured to act professionally. Moreover, there were two lecturers who believed they were using a lot of nonverbal strategies in class, yet the observational data suggest that this was not the case. The first case was Amy, who mentioned in the questionnaire and interview (as the above extract) that she found gestures and other nonverbal strategies easiest to apply and that this was the strategy she used the most. Her use of hand and body language as nonverbal strategies during speaking English were recorded eight times - only one-seventh as much as her most frequently used strategy. The other lecturer was Ellen, who shared: "I mostly use gestures and give example to make students understand instructions; and combine with translation to Vietnamese". However, when I observed her teaching, I only noted the use of nonverbal strategies six times during the approximately 30 minutes she used English in class; about one-third of her most frequently used strategy. This illustrates a mismatch between the lecturers' perceptions and practices in the use of the nonverbal strategy. 
According to the student survey results, lecturers' use of nonverbal strategies (e.g. miming, gestures, sound ...) ranked third (46.9\% responses) in terms of strategies students found helpful for their comprehension of lecturers' English speaking. Some students described other positive effects of lecturers' nonverbal strategies during the focus group discussions:

I like to study with the lecturers who have native-like pronunciation styles. For example, they speak naturally using gestures and body languages. This creates a friendly atmosphere and makes us want to reply to the lecturers. (IS5)

Besides, I think one reason why I lack learning motivation is that the lecturers' English speaking is uninteresting and unnatural. There are few facial expressions or gesturers in their speech, so I am bored and do not want to listen to her. (TS11)

The survey data and interview responses have demonstrated that a large number of students perceived nonverbal strategies to be useful for their comprehension and to have a positive influence on their learning motivations and behaviours.

In short, lecturers' nonverbal strategies were found to be used for the two main purposes of facilitating students' L2 meaning interpretation and getting students' attention to their L2 speech. In fact, both lecturers and students believed that nonverbal strategies would enhance students' comprehension and learning motivation. While some lecturers said they utilised a lot of nonverbal strategies, and this was the easiest strategy to use, the observation data found it to be in the group with the lowest occurrence. This can be because not all nonverbal behaviours performed by the lecturers were categorised as communication strategies, as described in 2.2.2.

\subsection{Humour}

The lecturers were found to use humour when they were speaking English to non-major students, albeit at a low frequency. The analysed data revealed nonverbal and verbal types of humour, which had two different functions. Nonverbal humour refers to multiple dimensions of "wordless and comic forms of expression that invite laughter and pleasure on the part of recipients" (Epure \& Lorena, 2018, p. 46). The lecturers mainly used nonverbal humour to make their L2 speech much more vivid, which, from what I observed, increased students' willingness to listen. Nonverbal humour was often combined with interactional and simplification strategies. For example, Amy sometimes did a little dance, combined with smiles and eye movements, when speaking English to enhance interaction and maintain attention among students. As for Olive and Una, they sometimes snapped their fingers before writing key words/phrases and simplified them on the board. In the cases described above, the lecturers applied nonverbal humour mainly to strengthen a simplification strategy and support an interactional strategy, which resulted in more interactive and drew students' attention to their 
English speaking. Noticeably, Amy whistled when time was up for an activity. This humorous behaviour was used many times in the same situation, making her whistling take on the meaning: "Time is up!" or "Stop!". In this case, humour conveyed a certain message when it was repeatedly used with the same meaning.

Along with nonverbal humour, verbal humour was also used by the lecturers during their English speaking. Ritchie (2010) defined verbal humour as a form of humour that involves the use of language as part of the joke. Unlike the nonverbal type, not all the lecturers used verbal humour. For example, when Ellen asked her students if they bought shares and securities, and saw them silent, she added "Do you? No (rising intonation) students have a lot of money (laughed)" (Class 2). This is called irony because the message contradicts the previous response and factual situation. Students normally do not have a lot of money and Ellen's irony evoked laughter. I labelled it a kind of communication strategy because it enriched the contextual meaning of lexical units used in the L2 speech and functioned as an input-enhancing tool. One more example was found in Polly's utterance: "I ask you what did you wear last Sunday? Nothing nothing nothing? (laughed) you covered your body with nothing" (Class 1), which broke the silence and caused laughter among the students. In addition to loosening the atmosphere, verbal humour was also used to encourage students' responses to lecturers' L2. This was the case when Polly used verbal humour to add excitement and invite replies from students. Specifically, she made a joke appealing to them on a personal level when she saw her class silent: "Can you? I love you, ok? Come with me and speak".

In line with the major goal of their teaching and characteristics of non-English major students, the lecturers used humour strategies to create a fun and relaxing classroom climate to encourage the students' listening motivation and willingness to communicate. Thus, the lecturers used humorous behaviours to encourage students to express themselves in English, even if it is wrong, with no fear of failure. Those humour strategies could have mediated the learning by making the students gain confidence in using the target language.

Although humour strategies were used by the lecturers with the lowest frequency, students perceived lecturers' sense of humour to be a motive for their willingness and engagement in their L2 speech (see 4.4.2). Students' voices also strengthened the above analysed positive effects of lecturers' humour:

When we start English lessons at 12.30 which is the first lesson in the afternoon, we normally feel sleepy and tired. Some teachers come into the classroom with a humorous sense and this makes us forget sleepiness. (IS6) 
We students are really excited to learn with the foreign lecturers who are funny and humorous. We feel listening to them more motivating and productive. (TS12)

The data suggest that humour functioned both as teaching and communication strategies since it did not just motivate students' L2 learning but also facilitated and encouraged the L2 communication between students and lecturers.

To sum up, the lecturers used humour in both verbal and nonverbal forms for different purposes. Nonverbal humour was used to clarify meanings of speech and create a more relaxing and friendly classroom atmosphere. By using verbal humour in the forms of short jokes or irony, the lecturers evoked laughter from students, which motivated students and increased their willingness to listen. Noticeably, the lecturers took advantage of verbal humour to encourage responses from students; and from what I observed it seemed to work. It is important for L2 teachers to encourage students to respond to them because this process will help identify gaps between what they want to say and what they can say (Swain \& Lapkin, 1995). From the best of my knowledge and research, humour has not been included in any communication strategy taxonomies so far. The identification of humour as a communication strategy that aims at enhancing students' L2 engagement and input comprehension, as well as encouraging their responses in L2, is a contribution of this study, which is further discussed in 6.1.3.

\section{Summary}

This chapter has presented three aspects of the lecturers' communication strategies: occurrence rate, their functions, and lecturers' and students' perceptions.

Firstly, this thesis has found that all the lecturers used all six strategies included in my initially proposed taxonomy. Humour emerged as a new strategy from the data. Self-repetition, codeswitching, and translation strategies were the most frequently used strategies with quite similar occurrence levels. Interactional and simplification strategies were used at a medium frequency, followed by nonverbal and humour categories, which were used the least.

Secondly, the lecturers used communication strategies to provide well-performed and correct language production, make L2 more comprehensible and accessible to students, draw students' attention and awareness to linguistic knowledge, enhance classroom interaction, create positive motivation, emotion, establish a relationship with students, and lastly to reduce students' L2 anxiety. Most of the functions of the lecturers' communication strategies were closely related to pedagogical, affective, and cognitive domains of language teacher awareness and knowledge. They seemed to be affected by different sociocultural and contextual factors. 
Lastly, the lecturers were fully aware of L1-based and repetition strategies, which was also reflected in the interviews and evidenced by the frequent use of those strategies in the observational data. The lecturers highly valued the importance of code-switching, translation, repetition, and simplification in their English speaking since they thought that would assure students' L2 comprehension. Those perceptions came from their pedagogical knowledge. As for students, while they believed that L1 and repetition were useful for their learning, they thought those strategies were sometimes overused and ineffective. Instead, they found other techniques such as simplification or non-verbal and humour strategies more helpful. 


\section{Chapter 6. Discussion}

In the previous two chapters (4 and 5), I have presented the findings related to the quantity and strategies of English communication used by the lecturers in non-English major classes. This chapter discusses the findings in relation to the literature and theory on English use in FL/L2 classes. The first part explores the complexity of language teacher roles in their practices and perceptions of communication strategies. Secondly, three mediation tools in EFL classroom teaching and learning regarding language use are discussed. The final section discusses the tensions between how lecturers see classroom English communication and how students perceive what works for them.

\subsection{The Complexity of Language Teacher Roles in the Use and Perceptions of Communication Strategies}

The complexity of the lecturers' awareness and perceptions in their use of CSs are analysed in this section, based on sociocultural, interactional, and teacher language awareness theories. Firstly, how the two domains of teacher language awareness are enacted via their use of CSs is presented. The second section is concerned with the role of the lecturers' pedagogical learner knowledge in their communication strategy decision-making. Lastly, the inconsistencies between the communication strategy typology used by the lecturers and the other models are analysed, which represents one of the main contributions of this thesis.

\subsubsection{The Two Domains of Teacher Language Awareness in Communication Strategy Practices}

There are three key roles of language teachers pertinent to language awareness: language user, language analyst, and language teacher (Andrews, 2007). As a language user, a teacher must be aware of their language performances to provide diversified and well-formed input for learners; hence, communicative language competence/language proficiency is essential (CelceMurcia, 2008). A language analyst must be capable of showing his/her knowledge of the language systems and of how the target language works; thus, metalinguistic awareness/content knowledge is required (Van Lier, 1998). The role of a language teacher enables him/her to make effective use of knowledge of pedagogical practices to facilitate students' learning; accordingly, pedagogical/procedural knowledge is vital (Andrews, 2007). With regard to teachers' use of communication strategies (CSs) in EFL classes, the results from this study have demonstrated a connection between the lecturers' language awareness as manifested in their roles of language users and language analysts, and their CSs use. 
Firstly, the analysis of the lecturers' use of CSs (see chapter 5) showed the efforts they made to teach English as a FL in non-English major classes, which draws on their roles of language users. For example, all the lecturers employed the self-repair strategy (see 5.1) right after they made grammatical mistakes to promote proper grammatical forms and create well-formed input for students. The lecturers frequently made self-corrections when their previous phrases were grammatically or lexically incorrect. They seemed to place priority on providing accurate language models by correcting their own language performances. Although those mistakes were insignificant and did not much affect the overall meanings of the utterances, those lecturers still decided to repair them so that the sentences would be accurate input samples. The lecturers' self-repair and paraphrasing practices are presumed to come from their determination to produce the best possible language models for students. In doing so, the lecturers appeared to have a certain level of language awareness in the user domain (Celce-Murcia, 2008) by monitoring their own language production in classroom oral discourse.

Secondly, the lecturers were seen to use the focus-on-form approach, which draws on their metalinguistic knowledge of the language system and belongs to the role of language analyst. For example, they used code-switching and translation to L1 to give further explanations or elaborations to syntactic units to raise awareness of students towards the use of those grammatical structures/expressions. Moreover, when speaking English, they intentionally repeated (self-repetition strategy) and emphasised certain salient language components by using fillers, word stress, and changing tones (interactional strategy) to raise students' awareness of a specific grammatical part of the speech (e.g. -ed ending on past tense verbs). The lecturers' techniques to emphasise certain language functions are linked to their high language awareness in the analyst domain. Those strategies are presumed to come from their content knowledge about "phonology, syntax, and discourse, an understanding of the nature of second language learning" (Anbesie, 2020, p. 51). Metalinguistic awareness is important for ideal language teachers, especially those who have learnt a language later in life and therefore have both explicit language awareness and metalinguistic knowledge (Van Lier, 1998). These two skills are particularly essential for language teachers since they are required to provide explanations for grammatical forms or usage norms to their students (Riordan, 2018).

In addition to focusing on forms, the lecturers also employed a meaning-focused approach by using questions, also referred to as probing, to check for students' comprehension (interactional strategy). Examples of such questions are "Why is this sentence wrong, students?" and "How do you understand irregular verbs?". Those questions are a form of probing that focuses on 
meaning during conversational situations that aim at drawing students' attention and awareness to how the target language system works, which again reflects the lecturers' role as language analysts. Their deep knowledge about how the target language works came from a higher language awareness, which is reflected in the use of the probing technique during classroom oral discourse to make sure students understand their instructions. These findings support Andrews (2001) in that teachers' high critical language awareness enables them to provide comprehensible input to students and monitor their own output so that it fits students' understanding. In order to do this, apart from a high level of subject-matter (or content) knowledge, teachers also need a high level of critical language awareness and discourse skills so that they can use such knowledge appropriately in the classroom (Andrews \& Lin, 2017; Parab, 2020).

From the discussion above, this study argues that language user and language analyst - as the two domains of teacher language awareness - are obviously manifested in the lecturers' practical use of classroom CSs. Those two roles are interconnected and work together as the lecturers intentionally used certain communication strategies when speaking English to nonmajor students. Interconnected here means that there is no clear boundary of each domain/role in the lecturers' use of communication techniques. For instance, the lecturers' focus-on-form and probing techniques could demonstrate their experiences as both language users and analysts.

While the first two domains of language teacher awareness were reflected in the lecturers' applications of communication strategies, the remaining domain - a language teacher - was found to be manifested in the lecturers' perceptions on communication strategies. The language teacher domain will be discussed in reference to pedagogical knowledge with its role in lecturers' perceptions and decision-making on the uses of classroom CSs.

\subsubsection{Pedagogical Knowledge and Perceptions of Communication Strategies}

The previous section discussed how the lecturers applied different CSs in the two integrated roles of language user and analyst. Findings of this study also provide evidence that the lecturers' perceptions about when and how communication strategies should be used mainly came from the previous learning experiences and practical knowledge they had developed in their teaching profession, which are referred to as pedagogical knowledge (Akbari \& Dadvand, 2014). 
It is evident that the lecturers used their own experiences accumulated from their teaching to put an emphasis on the significance of using certain communication strategies in their teaching practices. For example, two lecturers shared that they used a lot of L1-based strategies in response to students' reactions and behaviours during the lessons to make sure they would fully understand their instructions/directions. One lecturer's favourite choices of L1-based and repetition strategies came from her personal cognition about the goals of English education for non-English major students. From her perception, the final learning goal of those students was to understand the English linguistic knowledge required to pass the final tests and ultimately to graduate. Thus, the lecturers' professional experiences of classroom teaching and their practical teaching cognition are major factors underpinning their perceptions in the significance of those strategies, which aligns with previous research (e.g. Levin \& Wadmany, 2008; O'Bannon \& Thomas, 2014; Tobin et al., 2009). They seem to be part of the lecturers' experiential knowledge that had been developed over their teaching years. In other words, the lecturers oriented their decision-making more towards an everyday perspective that suited the teaching settings.

Sections 4.3.1 and 4.3.4 have indicated that the lecturers had critical local knowledge about the environment in which they were teaching, including information on students' limited proficiency and learning motivation or large class size, and perceived those elements as challenges and barriers to their English use. With their theoretical knowledge about language procedures and principles of language teaching, the lecturers developed and employed different strategies to increase the quality of input they produced and used appropriate content and presented that to the students based on the challenges (Andrews, 2001, 2007). In other words, they were responsive to their teaching context and students. The role as language teachers, pedagogical knowledge, and knowledge about learners and teaching environment also enabled them to anticipate the challenges their students faced regarding their L2 talk (Li, 2020; Parab, 2020; Wright, 2002). Thus, the lecturers perceived that using a lot of L1, repetition, and simple English could facilitate and enhance students' L2 comprehension and increase a positive relationship with students. While they all believed that the English-only instruction would benefit themselves, too, they decided not to apply it for fear that it might negatively affect their students' learning (see 4.2). Here it seems that English language lecturers' pedagogical knowledge connects with their learner-centred teaching (Yazdanmehr et al., 2020). When lecturers have deeper and more sophisticated pedagogical knowledge, they are more likely to 
direct their attention to their students' role in class and focus their teaching on students' needs, interests, or expectations.

Findings have also shown that the lecturers' own learning knowledge could have shaped their perceptions about the role of code-switching and translation strategies. As mentioned in 4.3.4, most of the lecturers expected their students to fully understand L2 structures and their speech, believing students' comprehension to be essential in language teaching. Thus, they all saw the positive role of L1 and hence used it in many situations. Those expectations and perceptions were likely to come from their former language learning and teacher professional training experiences. During their student and teacher training, they were mostly taught under the Grammar-Translation method which puts the primary focus on grammar rules and is taught using mainly L1 (Vu, 2019). Those lecturers had been taught by teachers who subscribed to a pedagogy style that gave priority to accuracy instead of fluency and put emphasis on grammatical competence over communicative competencies. Also, they had undergone formal training about second language acquisition and methods of English teaching, which could account for their theoretical support of an English-only approach. The lecturers in this research had degrees in English linguistics, were trained in English language teaching, and had opportunities to attend TESOL and linguistic conferences regularly. The formal education and training form lecturers' pedagogical knowledge system, which could have shaped their perceptions of the role of L1 use and CSs, as supported by previous research (e. Blume et al., 2019; Borg, 2003; Busch, 2010; Durán-Narváez et al., 2017; Farrell \& Guz, 2019; Moodie, 2016).

Lecturers' pedagogical knowledge comes from their own learning experiences and the practical knowledge have developed on the job, meaning the accumulated knowledge about content, culture, and political context (Akbari \& Dadvand, 2014). From my findings, I also suggest that knowledge about learners plays a defining role in shaping teachers' practical knowledge and is most influential in forming their pedagogical knowledge and beliefs about classroom CSs use. This kind of knowledge, also called pedagogical learner knowledge (Le, 2020), allows teachers to decide which language to use, and when, in order to ensure student comprehension and encourage students' language development.

Furthermore, knowledge is constructed via social interaction (Vygotsky, 1997), and individuals are not separable from the environments such as social and cultural contexts (Foster \& Ohta, 2005; Werani, 2018). Thus, other kinds of knowledge cannot be separated from the general pedagogical knowledge of English language teaching; those are not necessarily separate sets 
of knowledge, but are complementary and may inform each other (Li, 2020). Those arguments are strengthened in this study by the interrelated roles of knowledge about communication nature (User Domain), subject matter/ content knowledge (Analyst Domain), and pedagogical knowledge (Teacher Domain) in the lecturers' uses and perceptions of CSs.

In a nutshell, the lecturers' pedagogical intentions, decisions, and practices of classroom CSs are largely correlated with their language awareness and knowledge base. Knowledge about the nature of communication and the subject matter/content knowledge were embodiments of teacher language awareness, reflected via the lecturers' practices of CSs. Pedagogical knowledge as part of the teacher domain of language awareness shaped the lecturers' perceptions of CSs. Over the course of the lecturers' lifetime, their knowledge background and language awareness intertwine and together with the teaching settings and the lecturers' social and cultural milieus could have formed their communication strategy repertoire.

The above-mentioned complex process of how the lecturers' language awareness and knowledge shaped their practices and perceptions of CSs can be captured in a typology that illustrates how different CSs related to different factors. This typology is the focus of the last part in this discussion section.

\subsubsection{An Emerging Taxonomy from an Integrated Perspective}

This study found seven predominant CSs used by the lecturers during classroom English communication discourses: self-repetition, code-switching, translation, interactional, simplification, nonverbal, and humour. This study proposes the following five new basic functions within EFL lecturers' communication strategies: 1) to promote well-performed language production; 2) to enhance message comprehensibility; 3) to draw students' attention to and raise their awareness of linguistic knowledge; 4) to increase oral lecturer-student(s) interaction; and 5) to create positive affective effects (e. g. emotions, relationships, anxiety alleviation).

Also, the findings have demonstrated four basic discrepancies between the available CSs taxonomies (with a dominant cognitive/psycholinguistic/interactional perspective) and EFL lecturers' rationales grounded in pedagogical, cognitive, affective, and sociocultural influences.

Firstly, the available CSs typologies work from the perspective of solving communication problems; and those problems mostly come from the user who then applies CSs to tackle the problems. In this study, the communicative problems facing the user mostly come from the 
interlocutor (student) whom the CSs are aimed at. In other words, L2 communication strategies in teaching contexts arise when lecturers anticipate or realise the comprehension difficulty facing their students.

Secondly, the interactional perspective views CSs as a mutual attempt of two interlocutors to agree on a meaning (Faerch \& Kasper, 1983), showing the significant role of meaning negotiation. Findings from my research do not see much of a joint effort between lecturer (speaker) and student (interlocutor) to negotiate for meaning to reach communicative goals. Instead, the communication process is mostly initiated by the lecturer. For instance, after an utterance was made, the lecturer might realise that it was challenging to students' comprehension, thus, she tried to simplify certain words or the whole utterance. In other words, the CSs selection is more likely to be a one-way cognitive process by the lecturer as a result of his/her communicative competence and language awareness than a co-constructed activity involving both lecturers and students (Andrews, 2007; Celce-Murcia, 2008).

Thirdly, code-switching as a CS is considered as a problem caused by L2 deficit or linguistic shortcomings (e.g. Dörnyei \& Scott, 1997; Faerch \& Kasper, 1983; Poulisse, 1990). Codeswitching in this study is mainly related to the lecturers wanting to enhance students' learning and check for message comprehension, as well as to establish a positive relationship with the students. In this sense, the use of code-switching is mainly for pedagogical rather than communication breakdown purposes. Therefore, lecturers' use of code-switching as a communication strategy does not need to be viewed in terms of linguistic deficiencies but should be viewed from the dimension of a speaker' ability to overcome the difficulties in making himself/herself understood and making an effort to better engage the listener in the communication.

The first three inconsistencies suggest that CSs can be seen in terms of achievement and the modification/adaption ability of the user to achieve a mutual understanding, instead of being viewed as compensatory (e.g. Faerch \& Kasper, 1983; Poulisse, 1990) or problem-dealing (Canale \& Swain, 1980; Dörnyei \& Scott, 1995). The lecturer now plays a role as a mediator, negotiating the message whilst reconciling, settling, and compromising the meanings in his/her own cognition (Dendrinos, 2006).

Fourthly, as mentioned in 2.4, planning and consciousness are key defining elements in the CSs literature. This thesis evidenced that the lecturers' CSs were also moulded through their habitual speaking and teaching repertoires and used subconsciously. In fact, the selection of 
certain CSs (e.g. code-switching, self-repetition) is affected by the lecturers' practical teaching knowledge which is supported by sociocultural theory (see 6.1.2). Moreover, each learner is different and at a different level in their learning; therefore, lecturers, as mediators, have to use strategy tools to create a suitable context for each learner (Gao, 2013). Thus, in different communication situations, lecturers may use certain CSs subconsciously without planning.

One key contribution of this thesis is that it adds humour to the literature on teacher centric communication strategy taxonomies. To the best of my knowledge and research, so far about ten key taxonomies of CSs have been established, featuring about 35 types of CSs. Noticeably, none of them has included humour as a kind of communication strategy, although studies (e.g. Andarab \& Mutlu, 2019; Tunnisa, 2018) have confirmed the cognitive and affective benefits of using humour in EFL teaching. After all, humour is "an intrinsic component of the human language" (Andarab \& Mutlu, 2019, p. 24). Humour is also important in the language classroom as "comprehension of humour is a developmental ability" (Hidayanti, 2019, p. 10) which has a strong relation to both the cognitive and language development of an individual. This indicates that humour can be regarded as a cognitive aspect of communication, which may be one reason why it has been overlooked in the contemporary CSs taxonomies which mostly focus on a communication-based approach and describe CSs use from an interactional perspective. Although some researchers (e.g. Bialystok, 1990; Bongaerts \& Poulisse, 1989) took into account cognitive aspects, they devoted more attention to meaning negotiation and repair mechanisms in the CSs, ignoring the role of humour as a sense-making tool.

However, in the current study, humour emerged as a productive communication strategy from the analysis of the observational data. As mentioned in 2.2.2, different definitions of CSs have been proposed, depending on the scholars' perspectives. However, in the most general sense, communication strategies are conceptualised as a language-based attempt to prevent misunderstandings, establish meaning, and enhance the overall understanding and effectiveness of communication. In this study, some lecturers used humour to make their English speaking more interactive and/or attractive to students, which might simultaneously engage students' listening process and increase their readiness to respond to lecturers in English. Considering that conversations are bound to fail when an interlocutor does not listen, using strategies to engage them in a two-way communication is a first condition for a successful communication. From a sociocultural, interactional, and communicative teaching theory perspective, such a two-way communication accelerates language learning. This study found the lecturers used humour to draw students' attention and better engage them to L2, which thus 
could have facilitated students' L2 learning. Noticeably, two lecturers repeatedly performed their humour behaviours in particular situations, which made their humour convey certain messages, as the example of one lecturer's repeated use of whistling (see 5.7). The lecturer used this kind of nonverbal humour so frequently in certain situations that it was established the meaning of "Time is up!". In this sense, the lecturer's humour not only helps create a comfortable atmosphere of the class, reduce students' learning pressure and L2 anxiety, and better engage students; it also carries certain meaning during communication between students and the lecturer. Consequently, humour should be also treated as a communication strategy and added to EFL teachers' CSs typology.

There are some possible reasons to explain the above differences in the functions and characteristics of CSs. Because the lecturer had professional experiences of communication for years, their CSs performances represent a combination of both language awareness and language teacher knowledge base (content and pedagogical knowledge). Their use of CSs went beyond solving communication breakdowns but served to achieve communicative and pedagogical goals as a result of their interactional awareness which enabled them to estimate challenges posed for the learners (Andrews, 2007; Walsh, 2003). Meanwhile, their classroom behaviours were also affected by different sociocultural and affective factors. These partially account for the differences between the functions and characteristics of the communication strategies and for the recognition of humour as a new communication strategy in the current dataset.

The discussion above has highlighted four significant perspectives on communication strategies - cognitive, affective, pedagogical, and contextual - which have been overlooked by contemporary L2 CSs models. In fact, the traditional approach viewed CSs in terms of L2 proficiency (Tarone, 1983) whereas the interactional approach looked at CSs in terms of conversational adjustments (Faerch \& Kasper, 1983). Psycholinguists considered CSs in models of speech production and processing (Bongaerts \& Poulisse, 1989). These contemporary models seem to overlook the affective domains, disregarding the interlocutors' perspectives on what strategies work best for them. In addition, the context-based traits where communication happens are not taken into account by those models, which might lead to an overreliance on categories rather than functions (Burch, 2014). Although researchers (e.g. Bialystok, 1990; Bongaerts \& Poulisse, 1989) acknowledge that the choice to use a specific strategy is the result of cognitive processes and contextual factors, these factors are not captured by their proposed CSs typologies. Taken together, this study proposes an understanding of the 
complexities of different domains in teacher practices and perceptions of CSs use in the EFL non-major teaching context. With the typical roles of cognitive, affective, pedagogical, and sociocultural disciplines in EFL classroom communication, they all together constitute EFL teachers' CSs knowledge systems and form their CSs repertoires. Those lead to five specific functions of the CSs lecturers use in EFL classes, as demonstrated in Figure 6 below.

\section{Figure 6}

Disciplines and Functions of EFL Lecturers' English Communication Strategies

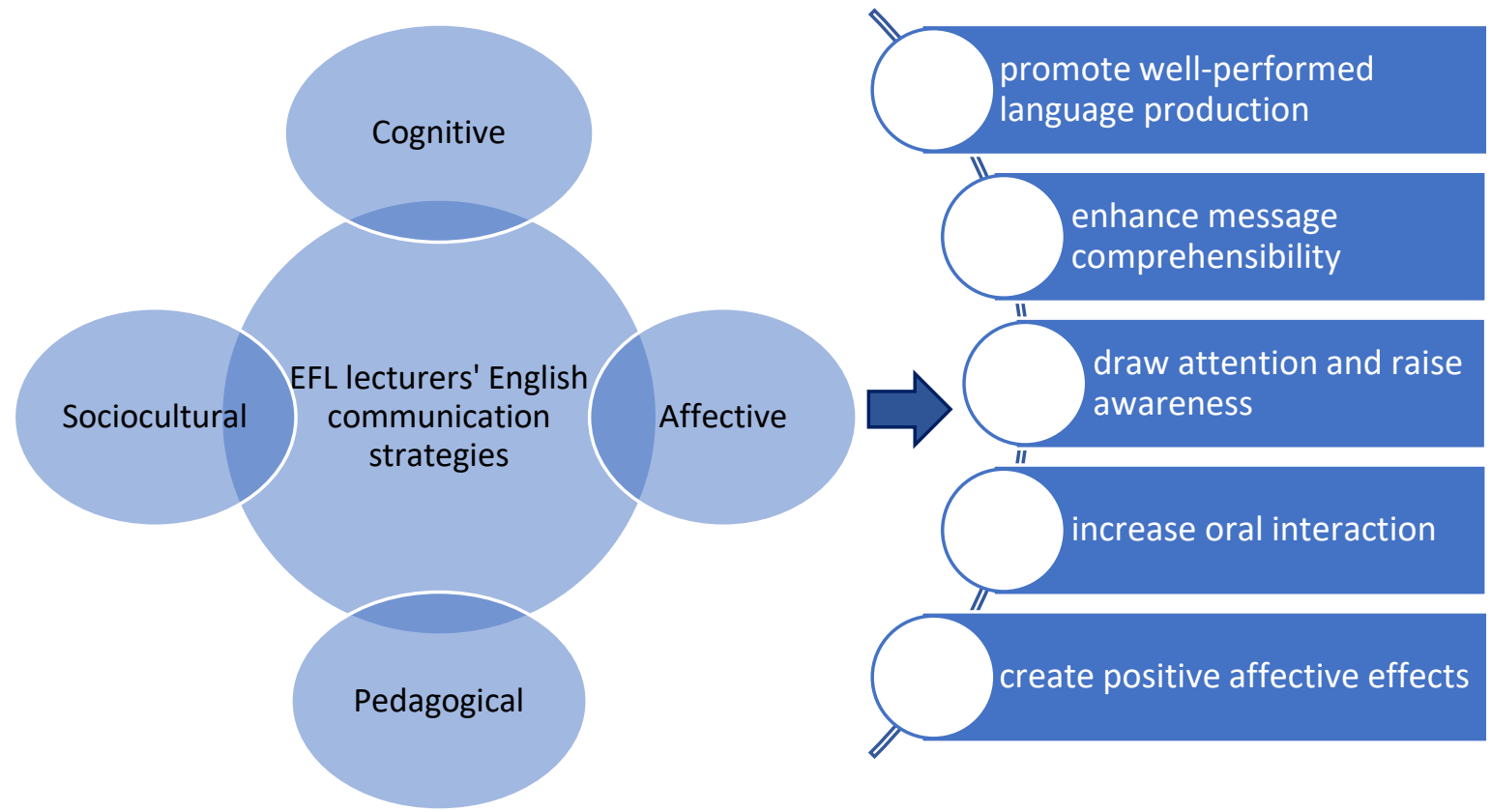

Language teacher awareness and knowledge base, especially pedagogical knowledge and classroom English proficiency, are vital in EFL teachers' practices and perceptions of CSs use in non-English major classes. Consequently, the taxonomy of CSs as used by the lecturers in this study was found to be distinctive.

\subsection{Mediation Tools in EFL Classroom Teaching and Learning}

Research suggests that L2 learning in classroom settings is influenced by both affective factors and the provision of opportunities to use the target language in interactions with others. Affective variables act as a filter that can prevent (negative emotions) or enhance (positive emotions) processing of the language input for efficient L2 acquisition (Krashen, 1988; Ni, 2012) while the opportunity to engage in classroom interaction facilitates acquisition by enhancing input and output in productive ways via meaning negotiation (Long, 1996). A positive student-teacher relationship and supportive learning environment have been shown to 
bring about positive effects for students' L2 learning, such as lowering L2 anxiety, enhancing students' willingness to communicate in L2, or fostering their learning strategies use (Joe et al., 2017; Ma et al., 2018; Nijat et al., 2019; Wei et al., 2009). In this study, these two affective issues also emerged to be salient in the perceptions of L2 learning expressed in the interviews as reflected in the identification of themes of less hierarchical relationship, concern and understanding, expectations, motivation, lecture presence, sense of belonging, corrective feedback, and face saving. The role of interaction as a mediator of learning was captured by the theme of classroom communication patterns. These assorted themes establish three major tools which are assumed as mediators in non-major EFL teaching and learning.

\subsubsection{A Positive Lecturer-Student Relationship}

The role of positive teacher-student relationships as a motive for L2 learning and teaching has been addressed and supported by research and theories in language teaching. Findings from this study have shown three main elements that were perceived by most of the lecturers and students to strongly affect the lecturer-student rapport in EFL non-major classes. They are symmetrical power, lecturer presence, and deep concern and understanding.

Symmetrical Power. According to students, a positive lecturer-student relationship is characterised by students' feeling that there is symmetrical or balanced power between them and their lecturer (see 4.4.4). In Vietnamese classrooms, strictly hierarchical and formal teacher-student relationships are the norm (Signorini et al., 2009) (see 2.5.3). This is clearly reflected by students' standing up to speak, which has been established as a component of classroom culture for a very long time. This rule was perceived by a majority of the interviewed students to reinforce the asymmetrical power structure of the classroom which positions the students at the bottom of the hierarchy. This caused them to feel stressed and worried, further increasing their reticence to use English in class. In addition, some students believed that their fear of face ${ }^{3}$ loss was amplified by having to stand up when speaking English since this act made them the focus of attention of both their lecturer and classmates. They felt that standing increased the degree of face loss should they make errors in their speech. Hence, the students hoped their lecturer would allow or encourage them to stay seated when they gave responses or answers. Students believed that being allowed to remain seated would reduce the hierarchical classroom feature and their fear of losing face would also be alleviated, resulting

\footnotetext{
${ }^{3}$ Face is commonly perceived as a person's image in social evaluations, and a subjective value in Vietnamese culture (Pham, 2014)
} 
in a better teacher-student relationship. Accordingly, students' English learning anxiety would also be reduced, which would help improve their willingness to communicate in English and use of learning strategies and thus could help them develop their English proficiency (Yashima, 2002).

Lecturer Presence. Data emerging from the interview findings (see $4.4 .3 \&$ \& 5.7) suggested that a sense of lecturer presence during classroom oral discourse facilitated students' English learning. Firstly, physical closeness was perceived by some students in this study to make them feel a sense of intimacy with lecturers, which supported their learning in that it increased their willingness to listen as well as readiness to give responses in English. According to the Interaction Hypothesis, when students are actively engaged in L2 listening and speaking, they are more likely to identify their L2 gaps and can better acquire the language since they simultaneously get input and produce output within their capabilities (Long, 1996). Secondly, some students perceived that lecturer presence could be expressed in their use of eye contact. In the complex and social context of classrooms where there are different individuals to communicate with, lecturers' eye contact is assumed to be an effective tool of social interaction. If lecturers observed students more during class, it would make the students pay better attention as well as allow the lecturers to see students' reactions (e.g. expressing nonunderstanding) and adjust their teaching approach accordingly. Also, through the use of eye contact, lecturers can create the feeling among students that "they are cared about and encouraged by their teachers" (Yang, 2016, p. 712). Accordingly, the use of eye contact can help minimise the social distance between students and lecturers. Physical closeness and eye contact during classroom communication reflect the value of lecturer presence, which can not only enhance a better interpersonal relationship but may also positively affect students' L2 development in that students feel more engaged and pay more attention to lecturers' L2 speech.

Deep Concern and Understanding. A successful lecturer-student relationship, as perceived by students, could also be established by lecturers' deep concern for and understanding of them. In this study, a majority of students regarded lecturers' personal interest in their ability to understand the lesson and their English use as the main factor that shaped their strong beliefs in communicative improvement and the main motive for their listening to lecturers' English (see 4.4.4). This demonstrates that when students are aware of their lecturers' concern for them, their learning needs are more likely to be met (Calhoun, 2019). This, in turn, is likely to decrease students' L2 anxiety and make them more comfortable to speak in English (Nijat et al, 2019; Wei et al., 2009). Along with concern, a lot of students indicated that 
lecturers' awareness of their aspirations and expectations regarding how English should be taught motivated them to improve and facilitated their L2 learning.

Findings of this study also demonstrated that lecturers' pedagogical decisions and their classroom language choice practices were closely related to their concern for students' comprehension and learning. Despite all the lecturers' high awareness of the need to minimise the amount of in-class L1 use as much as possible, they still used Vietnamese (L1) a lot and the main reason for this was their perception that students would comprehend L2 grammar better if it was explained in L1. Lecturers' L1 use was also a response to some students' inclass requests asking them to switch to L1. The lecturers' concern for students' comprehension might be rooted in the culture of EFL teaching in Vietnam where language acquisition has been predominantly developed by the Grammar-Translation method, which requires learners to memorise and accurately employ grammar rules. The question format of mid- and end-of term evaluation tests still prioritises structural and grammatical functions and neglects communicative skills (Ariatna, 2016; Rahman, 2017). This focus on grammar in assessment is also likely to impact the lecturers' decisions about using L1 to help their students better understand the grammatical structures for better test performances. This suggests that the Vietnamese testing and teaching culture influence EFL teachers' expectations and practices for students' learning ( $\mathrm{Vu}, 2019)$. In this study, those assessment expectations might have caused the lecturers' concern for students' comprehension of class content and their effort to ensure that students' learning was not compromised, which affected their teaching behaviours and practices of L1 use in class (Kazemi \& Soleimani, 2016).

The lecturers' concern for students not only affected their use of L1 but also motivated them to use a high proportion of English, despite some students' negative reactions to their English speaking. Thus, two lecturers tried to keep using as much English as possible in response to those students who were eager to listen and respond and who made a great effort to learn English for their future jobs. In this case, the students' positive learning attitude urges those lecturers to maintain a high proportion of English use in non-English major classes. This finding is in line with previous research which has shown that students are one of the main sources of motivation for EFL teachers (e.g. Gadella Kamstra, 2020; Javadi \& Mohammad, 2014). Students' behaviours, enthusiasm for learning, and positive attitudes directly influence L2 lecturers' classroom practices (Pourtoussi et al., 2018; Sugino, 2010). This is the case for the two lecturers in this study who felt motivated to maintain L2 use in response to some students' desire to learn. 
In addition to their concern for students at an interpersonal level, most of the lecturers perceived that a deep understanding of students' learning needs could positively affect their pedagogical use of English communication (see 4.3.1). Conversely, the lecturers believed that a lack of awareness of students' language levels, habits, and aspirations would leave them feeling unsure about how much and how fast they should speak English in class without negatively affecting students' comprehension, which can lead to a lack of the lecturers' confidence in their in-class performance. This suggests that the lecturers recognised the need to understand their students; and that they were willing to adapt their practices to better match students' interests and skill levels. Here it seemed that from the lecturers' perspectives, deep concern for and better understanding of learners' needs and interests could help them mould their language use to the class preferences, thereby optimising classroom learning.

This discussion has reflected the challenges and constraints of the English-only instruction in EFL contexts. On the one hand, acknowledging students' low English proficiency and worrying that the students would be frustrated if not able to understand their English hindered the lecturers' application of an English-only approach. On the other hand, the lecturers tried to use a lot of English since they knew that some groups of students expected them to do so for their communicative development. It was the lecturers' concern for different groups of students that created a tension in their application of an English-only practice and their ambivalence towards the use of this method. This, together with insufficient understanding of students, created an inconsistency between the lecturers' stated perceptions and their classroom practices. The lecturers' perceptions of the role of understanding students' expectations in creating more effective L2 practices supports the findings of Dang and Moskovsky (2019) that English-medium instruction can be successful when there is effective communication between policymakers, teachers, and learners; and modifications of teaching practices should be based on learners' interests and concerns.

Findings also showed that the lecturers used some communication strategies to develop a more positive relationship with students (see 5.2, 5.3, \& 5.7). For example, all the lecturers switched to Vietnamese when they wanted to encourage and comfort students who had made mistakes. Here, the lecturers' slipping into the L1 was a better way to show their feelings and empathy and create positive emotional states among students (Bruen \& Kelly, 2014; Caldwell-Harris, 2014). In addition, some lecturers utilised humour to alleviate students' L2 learning anxiety, draw students' attention, and improve students' L2 learning motivation, all of which were strategies that could have helped establish a closer relation between students and teachers 
(Hidayanti, 2019). Using Vietnamese and humour was both communicative and motivational aiming to minimise students' L2 anxiety, engaging them in L2 listening, and making them feel more confident to share their ideas in English. When students' L2 anxiety is lower, the L2 input in their language learning can be higher (Krashen, 1988). The positive emotions created by the lecturers' communication strategies could mediate students' L2 learning which might have helped them construct knowledge and develop skills (Swain, 2013). Thus, this study provides further evidence that teachers' communication strategies can contribute to a more effective teacher-student relationship which mediates student learning in that it enhances the input of language acquisition for the students and encourages students to produce output for better acquisition.

\subsubsection{A Supportive Learning Environment}

According to sociocultural theory, individuals and environments are intertwined (Foster \& Ohta, 2005). This implies that both physical and mental aspects of classrooms, as the environment where learning happens, can have a strong impact on students, either hindering or affording their learning. The findings emerging from data from the current study recorded how an encouraging learning environment can mediate student language learning. My study found three features that were perceived by students to constitute a conducive learning environment: the physical classroom layout, sense of belonging, and ways of providing feedback.

Physical Classroom Layout. A well-designed classroom space was perceived by many students to contribute to establishing a friendlier EFL environment for them (see 4.4.5). A comfortable English learning space was described by the students to have general classroom appearances such as posters and other visual decorations related to the English language. Such a classroom can stimulate students' EFL learning in that it can maintain students' learning persistence and create good learning motivation among them (Mutlu \& Y1ldırım, 2019). This is particularly necessary for non-English major students whose motivation with regard to learning English is normally low. This finding is congruent with Dörnyei’s (2001) motivational framework which emphasises the role of creating a basic motivational condition via a pleasant and supportive learning atmosphere for L2 learners. In addition, most of the interviewed students thought that movable desks and a U-shaped seat arrangement of English classrooms would better facilitate classroom communication in that it would improve visual engagement with the lecturer and help ensure that they could all hear better. With this desired classroom layout, the students could also have more opportunities to get physically closer to their lecturers, which could lessen the perceived hierarchical relationship between them. This 
physical layout is likely to mediate students' learning in that the students would feel free and more confident in speaking English in a supportive atmosphere, which is also important for the successful implementation of an English-only teaching approach (Dearden, 2014; Selvi, 2014). In other words, a favourably designed EFL classroom appears to better motivate non-English major students and better mediate their learning than a traditional one.

Sense of Belonging. Student achievement and engagement can be shaped and facilitated by school climate which includes both physical structures and social-emotional environment (Thapa et al., 2013). The social learning environment can include the relationship between teachers and students, and the ways they value each other's opinions may affect "the emotional climate for and of students" (Okello \& Olung'a, 2020, p. 69), as discussed in 6.2.1. Findings about a supportive English learning environment desired by many student participants have illustrated a close connection between physical and emotional learning environment. Students perceived supportive physical structures would bring them positive emotional states and connection within their classes, which implicitly reflects the students' sense of belonging to the classes (Strayhorn, 2018). Since these students are non-English major students, they might have a common feeling that the English subject is not relevant to their training or future job, which can lead to a lack of interest and motivation for English learning (Phan, 2011). Therefore, when the learning space creates a sense of classroom belonging for those students, they are more likely to consider themselves important parts of the class and are more likely to actively engage with the class activities (Museus et al., 2017). They can have positive affective responses (Tovar \& Simon, 2010) such as feeling more motivated, which has been found to correlate with L2 achievement and proficiency (Mantiri, 2015; Midraj et al., 2008; Santana et al., 2016).

The above findings align with Alhodiry (2016), who notes that external visual elements (e.g. photos of English proverbs) can create a strong sense of belonging to EFL classrooms for students. In addition, students' feelings of belonging to their EFL classes can also be strengthened if the behaviours and communication styles of lecturers convey to students the impression of being cared about, included, encouraged, and valued (Strayhorn, 2018). In this study, some lecturers used communication strategies to check for students' comprehension, correct errors, and encourage responses. By engaging in these communicative techniques, the lecturers showed their concern for students as individuals and tried to engage the students in the communication. Those strategies contributed to creating a sense of belonging to the English class amongst the students, which can improve their experiences as well as ensure their 
academic success (Cuellar \& Johnson-Ahorlu, 2016; Peacock et al., 2020; Thomas, 2012). Accordingly, both the physical set-up and social-emotional aspects of a classroom can impact how comfortable students feel in a classroom setting. The findings reveal that in tertiary EFL non-major classes in Vietnam, the physical appearance of the classroom and teachers' communication styles work to mediate and facilitate students' L2 acquisition.

Ways of Providing Feedback. The role of teachers' error correction during learners' oral production in second language acquisition has been a controversial issue. Since the communicative language teaching approach emphasises the role of fluency, it does not encourage the practice of error feedback, especially during oral discourse. However, from a sociocultural theory perspective, lecturers' correction of students' ineffective language to make sure the students learn and use positive and effective speech is necessary for the development of learners' inner L2 speech (Werani, 2018). According to the Interaction Hypothesis, explicit corrective feedback provides direct information about the grammaticality of the utterances that cannot be found in the input and therefore can be more effective than implicit feedback (Long, 1996). Based on findings from the student interviews on what helped them better learn English, this study suggests that lecturers' explicit corrective feedback is necessary for non-English major students' L2 development. Lecturers' explicit error feedback can help students identify their linguistic gaps and in that way they can learn from their own errors. This was also mentioned by some of the interviewed students (see 4.4.2).

However, too much corrective feedback can have negative effects on student motivation. Most of the students in this study believed that uninterrupted flows of speech would help them to save face in front of their friends and make them feel more comfortable and confident in speaking English in class. In this study, the matter of face was raised and emphasised by a lot of students who had a limited competency, demonstrating that face-saving is a quite common psychological characteristic in the EFL context. Thus, I suggest that lecturers need more effective ways of providing corrective feedback so that it will not interrupt students' oral performances and allow students to trust the teacher to treat them with respect and not to embarrass them in front of their peers. In other words, students' L2 learning anxiety and negative emotions (e.g. worrying of losing face) can be resolved when lecturers are more considerate of the ways they give corrective feedback to their students. As some students suggested, a good time for them to receive comments on their errors is at the end of the conversation and talk. This might be because this time they already complete their oral production; thus, they do not worry about losing face. Also, it seems that the students expect 
the lecturer to take advantage of some individuals' errors to teach the whole class so that all students will notice and avoid those errors. In other words, the issue of errors and mistakes should be treated as a problem that any learner can face and needs to be more systematically treated. By doing this, lecturers can create a calm, supportive, and respectful environment which can better mediate and facilitate their students' L2 learning.

Some of the above-mentioned attributes of a positive lecturer-student relationship and a supportive learning environment as perceived by the students in this study coincide with those of the motivational model proposed by Dörnyei (2001) (see 2.5.4). His framework of L2 motivational strategies has four main dimensions: creating basic motivational conditions, generating initial motivation, maintaining and protecting motivation, and encouraging positive self-evaluation. Those are divided into 20 specific techniques for L2 teachers to apply (e.g. making the teaching materials relevant for learners; increasing learners' satisfaction). Dörnyei's suggestion to encourage learners to personalise their classroom environment according to their taste aligns with students' perceptions regarding a well-designed classroom. Dörnyei's recommendation on appropriate teacher behaviours is manifested in lecturer presence with deep concern and understanding about students. He suggests patient and effective feedback will allow students to maintain a positive social image which contributes to better motivation. Similarly, Dörnyei also emphasises the role of a pleasant and supportive classroom atmosphere and increasing learner satisfaction in motivating L2 learners, which is further strengthened by the findings of this study.

\subsubsection{Interaction Opportunities}

According to both interaction and sociocultural theories in SLA, an EFL classroom should create a lot of opportunities for students to express themselves and engage in a variety of interactional and social contexts (see 2.1.1 \& 2.1.2). Under the communicative language teaching approach, students should be given opportunities to use and produce the learnt language by engaging in a wide range of $\mathrm{L} 2$ interactions and communicative tasks (Albahri et al., 2018; Owen et al., 2019). In order to do this, it is important that the classroom culture should avoid sustained monologues from the lecturer and instead adopt a dialogic communication model.

According to student participants, lecturers' talk often seemed too much like a monologue, meaning that the students became passive listeners (see 4.4.5). In fact, when lecturers dominate classroom talk, it means that there are fewer opportunities for natural and genuine interactions 
and communication involving the students. This one-way communication hinders learners' chances for meaning negotiation; thus, they cannot recognise many of their linguistic gaps (Long, 1996; Wei, 2012). Little engagement in meaning negotiation activities limits L2 acquisition because students then do not have many opportunities to make an effort to understand and express themselves using the L2 (Foster \& Ohta, 2005). A lecturer's domination of classroom talk also reduces social interaction (between student-student and/or student-teacher) which is important for language learning (Vygotsky, 1987). It also ignores the diversity of learners' language capabilities, limiting their interactive competence, and meaningful communication (Christianto, 2019).

When a lecturer engages in dialogue to exchange ideas and experiences with students or when students can interact orally with their classmates, students' L2 acquisition is enhanced. Dialogic situations not only help students get comprehensible input (via listening to the teacher) but also create opportunities to negotiate meaning (via communication strategies) (Long, 1996). This process of meaning negotiation draws the students' attention to the unknown features of the in-use language (Fang, 2010). The meaning negotiation and the attention obtained from interaction are assumed to help learners receive comprehensible input, identify ungrammatical structures and their linguistic gaps (via processing input and producing output), modify their output (via teachers' corrective feedback), and then reproduce better output. My study supports the idea that the input-negotiate-output process is an integration of many available second language theories and can be the most effective way to foster SLA.

Data suggests that students did not get enough opportunities to use English to interact in class and that lecturers did too much talking; thus, more dialogic practice is needed. Being engaged in many dialogic situations in L2, students can adopt the L2 linguistic patterns occurring in those situations and use them to mediate their L2 cognitive functions because language is the most powerful tool that mediates an individual's cognition (Lantolf et al., 2014). Moreover, students' L2 learning is mediated by their interlocutors, for instance their lecturers or more competent friends, who can provide useful scaffolding for them (Khaliliaqdam, 2014; Lantolf et al., 2014). This scaffolding is essential for EFL learning in that it provides students with appropriate input that is appropriate for their language level to better access the L2 content (Huang, 2020). Consequently, the more students interact with their lecturer and classmates in conversational and dialogical situations, the more they can construct their L2 knowledge and develop L2 skills. 
Minimising the dominance of teacher talk and opening up a two way line of communication with students, from teacher to students and vice versa, are characteristics of a student-centred class environment (Tsegay, 2015). Also, more dialogic practices are assumed to be prerequisites for more meaningful and genuine communicative situations, which would help ensure the success of the CLT approach (Alwazir \& Shukri, 2017; Christianto, 2019; Pham, 2007). However, students' low proficiency level is one of the challenges facing the practice of dialogic teaching in EFL classes (Haneda \& Wells, 2008). Therefore, to make dialogic practices work, teachers may need to use familiar genres of teaching talk such as repeating ideas and facts or using short questions to recall students' understanding (Mohammed Elhassan \& Adam, 2017). Some of those techniques are kinds of communication strategies, illustrating that teachers' communication techniques play an important role in creating classroom dialogic situations.

In short, a socially affective classroom culture has been shown to support L2 learning. In Vietnamese EFL classrooms, lecturers' choice of the language of transmission and their employment of communication strategies serves to express their concern for and develop an effective relationship with students and thus create a positive environment for students' L2 learning. However, there are other things they could do to ensure the transformation of knowledge to learners. It is essential for them to create an EFL classroom culture in which students can feel a strong sense of belonging, have positive emotional states, and engage in plentiful dialogic situations. Once the classroom culture is mediated by those affective factors and dialogic interactions, it can accelerate the transformation of L2 patterns in lecturers' English communication and students then acquire the L2 in a natural and more effective way.

Overall, findings of this study align with broader understandings about SLA in that it is a process that requires input, output, social interaction, open dialogue, and positive affective factors.

\subsection{Tensions between How Lecturers See Classroom Communication and How Students Perceive What Works for Them}

This section examines the tensions between lecturers' and students' perceptions regarding how classroom communication is best used in EFL contexts. Those tensions are presented in relation to three different aspects: the quantity and purpose of L1 use, the use of communication strategies, and the outcomes of teaching and learning. Once these tensions have been highlighted, the last part will look over the potential sources of the perceptual mismatches. 


\subsubsection{The Amount and Use of L1}

This study's findings demonstrate that the lecturers believed they used a large proportion of English in the classroom, which contradicted the observation and student interview data. This confirms that lecturers are sometimes not able to adopt practices that reflect their perceptions, as evidenced in various previous studies (e.g. Ansori et al., 2019; Badash et al., 2020; Ellis, 2012; Li \& Walsh, 2011). The overestimation could be accounted for by the complexity of real-life classroom contexts, the current proficiency level of students, or the difficult level of the lesson content. This is supported by the fact that the lecturers depended on different contextual and student-related factors to decide how much they used L1 and L2 (see 4.3.1 \& 4.3.4). The mismatch between the lecturers' perceptions and their own practices helps strengthen the view that lecturers' decisions on the amount of L1 and L2 use is based on context and is a matter that they must decide by themselves (Almoayidi, 2018; De la Campa \& Nassaji, 2009). Hence, there may not be a standardised 'fit' of proportions of L1 and L2 in EFL classes. It may instead vary depending on individual students and the classroom mix at that time as well as on what is being taught in the class that day. More importantly, these findings provide lecturers with an evidence-based impetus to engage in critical self-reflection on their teaching practices which may ultimately lead to adjustments to future teaching plans (see 7.7). This is particularly important when the CLT approach, which prioritises learners' opportunities to get maximal target language exposure, is a guideline for the lecturers in their teaching.

The results from this study also show a mismatch between the lecturers' practices and students' expectations regarding classroom language use. Specifically, the students were concerned about the balance between L1 and L2, expecting L2 to be used predominantly for all classes. They might be well aware of the scarce L2 exposure opportunities for them outside the EFL classroom contexts (Nation, 2003; Savaşç1, 2013); hence, they could come to English classes with the aspiration to be able to listen to and use English as much as possible. Also, they acknowledged some benefits of lecturers' English speaking (see 4.2), which made them desire their lecturers to speak a lot of English. The findings further support previous research (e.g. Anggrahini, 2019; Shuchi \& Islam, 2016) which concluded that the use of L1 should be as limited as possible and that English should be the main medium of EFL classroom communication.

Noticeably, however, students' perceptions matched those of their lecturers regarding when Vietnamese should be used in the classes. This means that both students and lecturers are well aware of the unrealistic expectation to eliminate L1, but they seem to agree on the point that 
any use of the L1 should serve a pedagogical purpose (Levine, 2014). In other words, the integration of the L1 into the language classroom should be done in a pedagogically principled way which does not diminish the role of the target language (Macaro, 2009). This again confirms the positive role of Vietnamese in English classes, especially in non-English major classes, where students' comprehension levels and learning motivation are often low. Hence, at least in Vietnamese EFL teaching contexts, where the implementation of an English-only approach has been constrained by different factors (e.g. teachers' limited competency, students' low proficiency, and negative learning behaviours) (Nguyen et al., 2016; Vu \& Burns, 2014), the exclusion of L1 seems to be impossible and undesirable. These findings align with those of previous research (e.g. Sevim \& Turhanli, 2019; Shabir, 2017; Shuchi \& Islam, 2016).

The lecturers' and students' agreement on restricting L1 use for strategic purposes is manifested in sociocultural theory, which supports judicious L1 use since it can mediate students' "understanding of task and content, which supports their co-construction of the target language" (Swain \& Lapkin, 2013, p. 110). Moreover, one general consensus in the literature is that judicious L1 use promotes rich communication and learning in the L2 (Levine, 2014). Hence, providing the L1 use is on-task, it may assist the process of target language input and output (Awada et al., 2020; Long, 1996; Nilubol, 2020). All the above-mentioned aspects have strengthened a principled use of L1 supported by both lecturers and students, giving way for a responsive bilingual approach in Vietnamese EFL teaching contexts (see 7.4, for more implications).

\subsubsection{The Use of Communication Strategies}

Generally, all the lecturers in this study valued the role of code-switching, translation, and repetition as ways to enhance students' comprehension and improve their L2 learning. Their perceptions matched well with the classroom observation and student survey data which showed that these strategies were the strategies most frequently used by them. Nevertheless, even though students all agreed that lecturers' repetition and L1-based strategies were necessary and helpful in some cases, they still thought that they were overused and ineffective. The lecturers' overuse of those strategies was, at least partly, because their use had become habitual and subconscious; in other words, lecturers' long-held perceptions might have led to some strategy uses to have become automatic in their teaching repertoires.

Once L1-based strategies are overused, they reduce the amount of L2 exposure for students in the classroom, which is an important setting for language input to EFL students (Gupta, 2004). 
The SLA literature generally indicates that L2 exposure is beneficial for enhancing students' English proficiency (Rahmadani, 2016; Rose \& Galloway, 2019). Specifically, a higher cumulative amount of L2 exposure is likely to prepare students for the high demands of using English in real-world interaction (Shvidko, 2018) and get them ready for work life (Ekoç, 2020). Hence, lecturers' overuse of code-switching and translation entails less L2 use and input to students, which can reduce students' opportunities to enhance their L2 proficiency. As for repetition, when lecturers repeat so much of what is already known and acquired by students, it is likely that the students will not need scaffolding and useful assistance which foster the development of language learning (Ohta, 2000). The overuse of lecturers' code-switching, translation, and repetition did not serve to enhance students' learning since students did not perceive that the use of these strategies helped them improve their English (see 5.3). Consequently, the overuse of these strategies created a mismatch between lecturers' and students' perceptions on their efficacy.

Overall, many students in this study had a positive attitude towards the nonverbal, interactional, and humour communication strategies. Specifically, according to the survey results, students believed that their lecturers' nonverbal behaviours helped their comprehension of spoken English the most (see 5.6). They believed that interactional and humour strategies motivated them to listen to and engage in their lecturers' L2 speech (see 5.4 \& 5.7). In fact, EFL students have a positive attitude towards nonverbal communication because it helps them enhance their English vocabulary and comprehension in learning an L2 and has a positive effect on the students' English achievement (Salimi, 2014; Sutiyatno, 2018). Teachers' use of interactional strategies also provides an opportunity for L2 learning and enhances learners' confidence (Pratoomrat \& Hiranburana, 2018; Walsh, 2011). Teachers' appropriate use of humour has been perceived by Vietnamese students to help hold their attention and enhance interest and memory in English classes (Pham, 2011). As mentioned earlier, non-English major students often have a low motivation and lack interest in learning English; thus, interactional, nonverbal, and humour communication strategies can better motivate them and enhance their learning. However, despite the students' positive perceptions of and expectations for the lecturers' more frequent use of interactional, nonverbal, and humour strategies, they were not commonly used by the lecturers. Data revealed that most of the lecturers did not mention much about those three strategies in their interviews, which might be because they were not well-aware of these CSs and thus employed them less compared with the others. 
All the above demonstrates a discrepancy between the lecturers' perceptions and practices of communication strategies and students' perceptions. On the one hand, the lecturers were fully aware and highly valued the role of L1-based and repetition strategies in enhancing students' comprehension and improving their L2 learning. On the other hand, students did not think such strategies were that helpful due to their overuse and negative effects (e.g. limiting their L2 exposure and interrupting the flows of L2 listening). Instead, students expected the lecturers to be more aware of their use of repetition and L1, and to prioritise the use of other strategies when speaking English to them. Students believed that L1-based and repetition strategies should only be the final options when other strategies did not work. Students' perceptions coincide with Bialystok and Fröhlich's (1980) and Dobao's (2001) observations that L1-based strategies are considered to be less likely to succeed in communication. However, students' beliefs on the effectiveness of repetition as a communication strategy appear to be incongruent with the study of Jensen and Vinther (2003) who found teacher repetition was an important condition for target language comprehension and performance. Additionally, while a lot of students expected their lecturers to use a lot of interactional, nonverbal, and humour strategies, those strategies were sparsely used by the lecturers.

To sum up, there were perceptual mismatches between the lecturers and students. The complexity of students' perceptions regarding what strategies were useful for students showed that lecturers face different tensions and challenges when using CSs. Despite a certain level of language awareness, there is evidence that the lecturers occasionally used code-switching, translation, and repetition subconsciously and habitually, which was perceived by students to be ineffective. Thus, as lecturers, they need to be more conscious and intentional when using CSs. Also, it is essential that lecturers have in mind certain strategies they should use more than others thanks to their effectiveness to students' learning. They need to be also creative and willing to experiment with different possible communication strategies to better meet students' needs.

\subsubsection{The Outcomes of Teaching and Learning}

As revealed from the research findings, it seemed to the lecturers that well-formed performances and abilities to make students comprehend language elements were important to successful language teaching. The lecturers' perceptions may be explained by their familiarity with the grammar and translation teaching method under which they were taught. In reality, many Vietnamese teachers are still familiar with this method because this is easy and makes them feel confident in teaching (Do, 2013; Ngoc \& Iwashita, 2012). This method puts an 
emphasis on learners memorising and accurately employing grammar rules (Vu, 2019), and as a result, the lecturers were likely to focus more on forms and right answers to questions than real-life conversations. In addition, most examinations and evaluation tests in English focus grammar and vocabulary and are mostly constructed in written form. The testing formats might have further cemented the lecturers' focus on explicitly teaching of grammar, hindering them from teaching English for communicative purposes. One other possible reason was that the lecturers might have used their own practical knowledge and personal cognition (see 6.1.2) in perceiving the learning goals of non-English major students. They noticed students' low learning motivation and they knew that some students were studying English just to pass tests and examinations. This could have supported the lecturers' perceptions that students just wanted to pass the tests, and as a result they prioritised grammar teaching or the use of the target language. All of those factors could have created tensions for the lecturers when they had to balance the students' needs for academic achievements and the principles of the innovative CLT approach.

From the students' perspectives, the ability to speak English fluently and use English for future jobs are two important learning outcomes (see 4.2.2). In fact, while the students perceived lecturers' use of L1 as necessary for their understanding, they also believed that language teachers should have native-like speaking styles (e.g. pronunciation, intonation, stress ...). This supports previous studies (e.g. Ellen \& Taverniers, 2011; Tergujeff, 2013) which indicate that many students perceive native speaker-like pronunciation as an ideal goal. In this study, the students in school A had opportunities to be taught by a volunteer from Australia. A lot of students in both schools wanted to improve their English skills; thus, they had learnt or were learning English at private evening foreign language centres where they were taught by teachers who speak English as their L1. Hence, it is possible that they could compare between their lecturers' English and the English spoken by English L1 people, leading to students having higher expectations of their lecturers' speaking skills and of their own language learning.

Meanwhile, a lot of students in this study emphasised the importance of how English lessons linked their future jobs, which had been identified as a central motivation types among nonEnglish major students (Chairat, 2015). In other words, students wanted to learn about the words/phrases, as well as oral practices, related to work situations they might encounter in the types of jobs they hoped to do in the future (see 4.2.2). According to those students, one of the reasons for their lack of interest in listening to lecturers speaking English was the speech 
content which was mostly textbook-based and outdated. It seemed that they needed their lecturers to be more creative in using the textbooks and to present content that was linked to their future jobs. Generally, all Vietnamese non-English major students have English lessons for specific purposes which are designed to be close to their training sectors/majors. Those lessons normally run in the final year or semester of their whole training course; and they mostly aim at improving vocabulary and reading comprehension skills. A lot of students in this study thought that those lessons were inadequate and outdated in content and not very helpful for them. Some students shared in the interviews that they expected the English for their professional purposes to be thoroughly integrated in all English lessons. This has some implications for the curriculum and syllabus designers (see 7.6).

While the lecturers believed that teaching should aim at providing students with adequate grammar knowledge, vocabulary, and the skills needed for excelling in tests and examinations, the students' learning goals were to be fluent in English speaking and to be able to use English to communicate in their pending jobs. In the Vietnamese education context, students are required to take many courses and lecturers are expected to teach them. As a result, both lecturers and students are overworked, which is likely to create these tensions (Huynh, 2017).

As can be seen from the three discussion sections in this chapter, the lecturers' and students' perceptions differ with regard to how English communication in the classroom can create language learning opportunities. Those perceptual mismatches might have occurred because the lecturers and students had different ideas of what constituted a learning opportunity and what the learning goals were. Since perceptual mismatches are prevalent in educational settings (Khodamoradi et al., 2019), they need to be identified and managed instead of avoided (Lee \& Choi, 2019). If both lecturers and students recognise the mismatches and understand the underlying sources, such mismatches may even provide a new learning opportunity (Kumaravadivelu, 2003).

\subsubsection{What Might Cause the Perceptual Gaps between Lecturers and Students?}

Findings have shown two aspects of perceptual mismatches between lecturers and students: they did not seem to fully know what they expected from each other and they had different perceptions on how classroom communication could construct learning opportunities. These differences caused tensions within the classroom and ultimately led to fewer opportunities for classroom communication and learning for students, resulting in both lecturers' and students' expectations not being fulfilled. Moreover, these mismatches suggest that the lecturers' 
approaches to classroom English communication were not perceived as useful by students. All these mismatches in expectations can lead to low levels of student engagement, motivation, and trust (Lobo \& Gurney, 2014), which is likely to have a detrimental impact on the lecturerstudent relationship and negatively influence students' learning outcomes (Cuéllar \& Oxford, 2018).

Both qualitative and quantitative data from the lecturers and students have confirmed a lack of understanding at the interpersonal level between lecturers and students, despite the lecturers' deep concern for students. This suggests that insufficient understanding may be one of the main reasons for the mentioned perceptual mismatches between the lecturers and students. Thus, both the lecturers and students expressed the perception that little personal connection and communication between them were barriers to their teaching and learning (see $4.3 \& 4.4$ ), which created a lack of mutual understanding between them regarding the teaching and learning English. Two lecturers commented that their use of classroom English could have been more effective had they been more confident and better prepared. Hence, it is likely that the lecturers did not know how much L1 and L2 their students expected them to use, leading to those mismatches between students' expectations and lecturers' practices.

More importantly, the perceptual gaps might not only come from lecturers and students, since L2 learning is also embedded in the cultural and social aspects (Dixon et al., 2012). In fact, sociocultural factors are likely to be important sources of those perceptual gaps. In Vietnamese society, the teacher-learner relationship is considered particularly hierarchical and formal (Signorini et al., 2009), which is related to Vietnamese Confucian heritage. It is a culture where learners "are inculcated with a fixed set of attributes such as respecting the teacher" and strictly following what the teacher expects them to do (Nguyen, 2019, p. 24). Students are accustomed to keeping silent; and there is often little interaction between teachers and students, let alone interpersonal communication. Despite recent innovations and reformation in Vietnamese education systems in general and EFL teaching in particular, studies indicate that traditional hierarchical structures are still prevalent in Vietnamese classrooms today (Humphreys \& Wyatt, 2014). This can hinder the successful development of an interpersonal relationship between lecturers and students. This is supported by the findings of this study in that the students believed that changes to the seat arrangements and removing the expectation of having to stand up to give responses to the lecturer would enhance a sense of equality and establish a less hierarchical relationship. Students also wanted their lecturers to know more about them. The establishment of a personal connection was hindered by a lack of interpersonal engagement 
both inside and outside the classroom. Consequently, the social and cultural factors specific to the Vietnamese context are likely to cause a formal, hierarchical relationship, leading to a lack of interpersonal communication that causes perceptual gaps between lecturers and students.

To sum up, insufficient understanding caused by a lack of connection and communication between lecturers and students at the interpersonal level and the perceived lecturer-student hierarchical relationship are the two main reasons for the tensions and inconsistencies between the lecturers' and students' perceptions of learning opportunities and purposes. The issues appear to be linked to the prevalent Vietnamese learning and teaching culture which emphasises hierarchical structures that discourage interpersonal connection.

\section{Summary}

This discussion chapter has integrated and discussed the different research findings, linking them with the research aims and theoretical framework. Three different aspects surrounding classroom English communication have been examined in depth. This thesis has found that the two roles (User and Analyst) of teacher language awareness are interconnected and pivotal in realising the synthesis between language awareness and communication strategy practices. Lecturers' roles as language teachers together with pedagogical learner knowledge and other affective and sociocultural factors shape their perceptions about how to use communication strategies in class. Based on the research findings presented here, a new CSs typology with distinctive purposes and characteristics is proposed. This research has also identified a positive lecturer-student relationship, a positive learning environment, and interaction opportunities as three key mediation tools in EFL learning. These findings emphasise that the affective and interactional domains are important mediators in non-English major EFL classrooms. Lastly, lecturers' and students' perceptions of three different aspects of English communication in their classrooms are reviewed and discussed. Generally, except for a principled L1 use and benefits of an English-only approach, there are few matches between the lecturers' beliefs and the students' perceptions of how classroom English communication can create language learning opportunities.

Based on all the evidence presented, this thesis identifies a lack of understanding due to limitation of communication at the interpersonal level between the lecturers and students as a major source of the perceptual mismatches. However, the underlying reason for the lack of understanding and communication could be due to the hierarchical and formal lecturer-learner relationship, as part of the Vietnamese culture. Overall, the data suggest that an overhaul of 
current learning and teaching practices aimed at reducing hierarchical classroom structures, increasing opportunities to use L2 for students, and raising students' and teachers' awareness of the others' expectations as outlined in this chapter would enhance the overall efficiency of EFL teaching and learning in Vietnam in general and non-English major classes in particular. 


\section{Chapter 7: Implications, Recommendations, and Limitations}

The findings and discussion of this thesis have led to a number of implications and recommendations for EFL teaching and learning at the two research sites as well as for other similar contexts in Vietnam. This closing chapter firstly recaps how the findings addressed the research questions. It then presents the seven main themes; they are communication strategies; classroom dynamics; perceptual gaps; language policy; syllabus and testing; teacher selfreflection and students' feedback; and teacher education and competence standardisation. The last two parts describe the limitations of this research and present recommendations for future studies. This chapter ends with a brief conclusion of the whole thesis.

\subsection{Addressing the Research Questions}

The investigation of EFL teaching approaches in Vietnamese non-English major tertiary classes presented in this thesis was framed and guided by four research questions. Table 7 below presents a summary of the key findings for each research question:

\section{Table 7}

Answers to Research Questions

\begin{tabular}{|c|c|}
\hline Questions & Key findings \\
\hline $\begin{array}{l}\text { 1. How much } \\
\text { Vietnamese versus } \\
\text { English do lecturers use? }\end{array}$ & $\begin{array}{l}\text { - Observation and students' interview data demonstrated that lecturers spent } \\
\text { marginally less time speaking English than Vietnamese. } \\
\text { - Lecturers reported using more English than Vietnamese in interviews. } \\
\text { - The majority of students expected their lecturers to use English predominantly for } \\
\text { all lessons. }\end{array}$ \\
\hline $\begin{array}{l}\text { 2. What English } \\
\text { communication strategies } \\
\text { do lecturers use and how } \\
\text { are they used? }\end{array}$ & $\begin{array}{l}\text { - Lecturers in both sites were observed to use seven main types of communication } \\
\text { strategies (presented in order from the most to least frequently used): self- } \\
\text { repetition, code-switching, translation, interactional, simplification, nonverbal, and } \\
\text { humour. } \\
\text { - Most lecturers perceived nonverbal strategy to be easiest to apply, but it was } \\
\text { among the least used in class. }\end{array}$ \\
\hline $\begin{array}{l}\text { 3. What are lecturers' } \\
\text { perspectives on what } \\
\text { affects their English } \\
\text { communication in } \\
\text { classes? }\end{array}$ & $\begin{array}{l}\text { - Lecturers revealed three different elements which affected their English speaking } \\
\text { in non-major classes: student-related, teacher-related, and contextual factors. } \\
\text { - Student-related factors, including their level of English proficiency, learning } \\
\text { habits and motivation, and lecturers' concern and understanding about students } \\
\text { were perceived by lecturers to be the most influential. } \\
\text { - Lack of understanding about students' needs and interests were also believed by } \\
\text { lecturers to strongly affect their pedagogical practices of classroom oral } \\
\text { communication. }\end{array}$ \\
\hline $\begin{array}{l}\text { 4. What are students' } \\
\text { perspectives on what } \\
\text { helps them better } \\
\text { understand their } \\
\text { lecturers' English } \\
\text { speaking and learn } \\
\text { English more } \\
\text { effectively? }\end{array}$ & $\begin{array}{l}\text { - Students revealed four different elements which affected their comprehension of } \\
\text { lecturers' English and learning motivation: student-related, teacher-related, } \\
\text { affective, and contextual factors with student-related and affective elements being } \\
\text { the most influential. } \\
\text { - Students believed lecturers' interactional, simplification, and nonverbal strategies } \\
\text { to be the most helpful to their English comprehension and willingness to listen. } \\
\text { - Students thought that lecturers' concern and understanding about their needs, } \\
\text { communication strategies and styles, hierarchy-free classroom, well-design layout } \\
\text { of classrooms, and many interactional opportunities could enhance their learning } \\
\text { motivation and mediate their English learning. }\end{array}$ \\
\hline
\end{tabular}




\subsection{EFL Teachers' Communication Strategies in Non-English Major Classes}

The findings from both the qualitative and quantitative data of this study regarding the lecturers' use of CSs during classroom oral discourses and the students' perceptions of CSs (see chapter 5, sections $6.1 \& 6.2$ ) carry some implications for lecturers' use of L2 CSs in Vietnamese EFL non-major classrooms.

Firstly, the data suggest that lecturers' use of diversified interactional and nonverbal strategies is particularly beneficial to students. For instance, when speaking with L2/FL speakers, using fillers can help students maintain the conversation, which is a very valuable skill for them to learn because they can benefit from receiving additional modified input (Larsen-Freeman \& Long, 1991) even though they cannot find certain solutions due to their limited linguistic competency. Maintaining a conversation is argued to be particularly important for low proficiency speakers since keeping a dialogue going allows time for scaffolding or mediation from their more competent interlocutors (see 2.1.1). More importantly, CSs can guide students to prompt their teachers who will then modify the students' utterances. This can be applied when students communicate with native speakers of the language they are learning since the use of CSs can help the students to continue their conversation and have more opportunities to improve their interlanguage systems (Mariani, 2010). Consequently, it is recommended that CSs should be explicitly introduced to EFL students, which will equip them with the necessary knowledge, consciousness, and confidence to deal with real-life L2 communication.

Secondly, the findings demonstrate that lecturers' communication strategies may have a great influence on students' willingness to listen to their L2 speaking (e.g. nonverbal strategies) and create comprehensible input for SLA (e.g. simplification). Also, certain techniques in lecturers' CSs can be noticed and transformed into students' knowledge with the mediation of different sociocultural factors (e.g. positive emotional state) (see 6.1.4). This implies that lecturers' classroom CSs provide comprehensible input valuable for students to follow/learn and lower the affective filter, which is necessary for non-English major students whose learning motivation is low (Chairat, 2015). However, the observation and student interview data demonstrate some instances when the lecturers used CSs, particularly translation and repetition, out of habit (Gass \& Selinker, 1994; Kárpáti, 2019). Thus, it is imperative that lecturers be better aware of and use a variety of CSs, particularly nonverbal and simplification strategies in different classroom communication discourses to provide comprehensible input and good models for students to follow and learn from. 
Thirdly, it was found that CSs were used by the lecturers in this study for different purposes: scaffolding, mediation, comprehension enhancement, engagement encouragement, and creating positive emotions and learning motivation amongst students. Those strategies do not merely aim at enhancing communication but also facilitating students' overall learning process. Obviously, lecturers use language to communicate lesson content, teaching activities, and other pedagogical intentions, and CSs support them to do those teaching tasks in more effective ways. Thus, it is argued that communication, motivational, and teaching strategies can be interwoven and mutually supportive in increasing the overall efficiency of L2 teaching. This means that, in some circumstances, there are no clear boundaries between teaching, motivational, and communication strategies. Also, as L2 learners, lecturers in this study use CSs to assist their own L2 fluency. Thus, it is recommended that examining the role of teachers' communication strategies requires a more mindful consideration of classroom contexts and that there needs a new framework for EFL teachers' classroom communication strategies.

The data in this study has shown that lecturers' use of English CSs in EFL classroom discourses are restricted in scope yet complex in function and motivation. Lecturers' CSs can be both conscious and subconscious, multi-functional, affected by different factors, and sometimes intertwined with motivational and teaching strategies. It is, therefore, recommended that lecturers' use of CSs in the classroom should be examined in a multi-faceted dimension.

\subsection{EFL Classroom Dynamics for Non-English Major Students}

Sections 4.3, 4.4, and 6.2 have shown that non-English major students perceived a successful lecturer-student relationship, encouraging learning environment, and interactional opportunities to positively affect their emotional state and L2 learning motivation. The findings imply that Vietnamese EFL classroom dynamics, including both physical and emotional aspects, contribute to SLA by creating L2 opportunities and facilitating L2 knowledge and skills, particularly for non-English major students.

From the students' perspectives, a well-designed and favourably decorated classroom would make them feel that they were in an English rather than another type of class. This suggests that a physical classroom layout can foster a greater sense of belonging to the English classroom, which can help develop emotional engagement and promote their academic competence (Buhs et al., 2006; Hernández et al., 2016; Korpershoek et al., 2019). Students also perceived psychological dynamics of EFL classrooms (lecturers' patient and systematic corrective feedback, lecturer presence, more symmetrical power structures, and more 
interaction opportunities) to create positive emotions among them. Students' positive emotional state created by those classroom dynamics can determine the success of the transformation from external speech to inner speech (Swain, 2013). Lecturers' communication behaviours, including language use and communication strategies, function as external and social speech which are the models for students to follow (Werani, 2018). The positive emotional states can mediate the students' cognitive functions; thus, they may actively perceive those communicative behaviours in their mental process, and naturally move them to their inner speech (Vygotsky, 1987) (see 2.1.1). Since the inner speech can boost students' attention (DiCamilla \& Antón, 2004), they then become better at noticing lecturers' communication styles and strategies. Students will then plan consciously (Lantolf \& Thorne, 2007) and then feel that lecturers' communication behaviours are worth noticing and thus they may gradually integrate the observed behaviours into their knowledge and skills.

EFL classrooms should offer features of a supportive and motivating environment, both physically and emotionally, which will encourage more interactive exchanges and social engagement to better facilitate the communicative language teaching approach and improve learning. Burgos and Molina (2020) argued that introducing some changes in English tertiary teaching is necessary, which is further supported by the findings of this study. The dynamics happening in a classroom need to be significantly improved to better response to the communicative and student-centred EFL teaching directions (Kweon \& Spolsky, 2018). Classrooms need also have features which better respond to students' expectations and needs since they are the main entities of the learning process. In realising this, this study emphasises the essential roles of affective and relational factors in which lecturers with their communication styles and behaviours play a central role.

Research results also imply that lecturers' classroom communication strategies and certain behaviours during oral interaction with students play a central role in shaping good class dynamics and opening more L2 learning space. Specifically, an increased use of interactional techniques (e.g. checking for comprehension or asking for clarification) can give more opportunities for students to ask for further clarification from lecturers to enhance their understanding and can create more dialogic and interactive types of discourse. Meanwhile, students perceived lecturers' nonverbal and humour behaviours to be friendly attributes that could help them feel less stressed and more motivated to learn. This implies that an increase in the use of nonverbal and humour techniques by lecturers could strengthen the relationship between them and their students, since students might then feel that their psychological needs 
are met by the lecturers (Calhoun, 2019; Smit et al., 2014). Lecturers' provision of retrospective feedback can be an expression of respect for students' talk by the lecturers, which will alleviate the issue of face loss and anxiety and thus lower the affective filter allowing input to freely pass so as to be acquired. Lastly, being more considerate of physical distance and frequent use of eye contact with students will help lecturers create a more satisfying learning experience for them.

A reorganisation of EFL classrooms to create a less hierarchical setting and encourage interaction is highly recommended. Establishing a safe and positive affective learning environment should be the main duty of EFL teachers who should make better use of communication strategies and classroom behaviours to strengthen lecturer-students relationship and thereby positively influence learning in direct and indirect ways (Frymier \& Houser, 2000). It is also suggested that the issue of EFL classroom appearances needs to be taken into account by institutional leaders and stakeholders so that the classroom can create more positive emotional effects to learners. Taken altogether, the classroom dynamics as perceived by non-English major students in Vietnamese EFL classes is closely related to both the physical, relational, and emotional domains in which the role of lecturers' communication strategies and styles is pivotal.

\subsection{Perceptual Gaps in Non-major EFL Classes}

Section 6.3 has demonstrated the perceptual mismatches between lecturers and students regarding how classroom English communication is best used in EFL contexts. The two significant causes of those tensions are a lack of understanding at the interpersonal level between lecturers and students and the cultural and social aspects of Vietnamese classrooms. Accordingly, it is implied that a student-lecturer co-constructed teaching approach and reorganising the classroom physical layout can help alleviating the perceptual gaps in Vietnamese EFL classes and enhance students' L2 learning.

One of the first things that EFL lecturers could do is to reconcile the contradictory poles between them and their students so that they are both simultaneously teachers and learners (Freire, 1970). Lecturers need to be learning from the students what the students need to learn and what helps them to learn, whilst students can serve as teachers in informing the lecturers in this regard. For this collaborative learning, discussion is a good way for lecturers to demonstrate their commitment to a more egalitarian teaching model and for students to outline their ideas for an ideal learning environment and conditions that best suit them (Brookfield \& 
Preskill, 2012). Via regular personal conversations or informal group discussions between lecturers and students, the lecturers might be able to learn about students' aspirations and expectations so that they can apply the practices and teaching techniques that better match the students' expectations and needs. As for the students, they can also gain a better understanding about what their lecturers expect from them, and how their lecturers feel about their classroom behaviours. This can help the students have more positive and responsive behaviours to better motivate their lecturers' teaching. Therefore, a better mutual understanding can benefit both lecturers and students and is argued for more effective student-centred teaching.

Also, as lecturers, it is important to make sure students feel free to share their views during their conversations. Firstly, it is essential for them to be aware of possible cultural barriers that may hinder their students from expressing their personal views. For instance, two students in this study said that their lecturers used to ask about their preferences in front of the whole class, but only a few students were confident enough to raise their voice (see 4.4.4). This reticence to talk is likely tied to the Vietnamese teaching and learning culture which means that most of the students were reluctant to raise their personal viewpoints related to their lecturer when being asked in front of the whole class as they might fear being evaluated or criticised by their lecturers and classmates. When being fully aware of those hindrances, lecturers can try different ways to work out how to have a better understanding about students' perspectives regarding what works best for them (e.g. amount of L1 and L2; what strategies are helpful). When realising that asking students during classes does not work well, lecturers may find other chances (e.g. break or out of class time) and other means (e.g. via email or social media) to get to know about their students.

Moreover, at the school level, one other measure might be taken to alleviate those perceptual gaps. In Vietnam, the physical setup of most classrooms follows teacher-fronted and teachercentred direction (Sullivan, 2000). This physical layout, as expressed by some students, created a power distance between the lecturer and students, hindering the development of a positive interpersonal relationship and classroom communication. Hence, reorganising the physical layout and redesigning appearances of the English classrooms can create basic motivational conditions for students in EFL learning (Dörnyei, 2001) because it can make students feel freer and more comfortable to share their voices with lecturers.

Consequently, lecturers have been shown to play a decisive role in bridging those perceptual gaps. It is recommended that lecturers need to find out from students what the students want to learn and what helps them to learn that. To do this effectively, lecturers need to develop a good 
awareness of individual students' cultures, make an effort to allow students to teach them, and show respect for students. Thus, teacher language awareness should be lifted to highly critical language awareness which integrates different roles and knowledge bases, particularly pedagogical learner knowledge (Le, 2020; Li, 2020).

\subsection{Language Policy in Non-major EFL Teaching}

This study has demonstrated that an English-only teaching approach is not a wholly suitable practice in EFL non-major contexts because both lecturers and students rely on the use of L1 for effective language teaching and interpersonal functions. The data also show that they both agreed and supported the maximal use of L2 for the benefits of L2 proficiency maintenance (for lecturers) and development (for students). Thus, when an English-only policy is believed to be inapplicable, an English-dominant approach is suggested to be an effective alternative in non-English major classes.

Findings from this study (see $4.1 \& 4.2$ ) imply that L1 (Vietnamese) should not be expelled from EFL classrooms, but on the contrary, L1 is an effective means to motivate EFL students and to better their L2 learning. As for the low proficiency English level students like those in this study, L1 can help them have a better understanding of abstract vocabulary and raise their consciousness of complex grammars/structures (Alijani \& Barjesteh, 2018; Arshad et al. 2015). In addition, non-English major students tend to have low learning motivation and are likely to have L2 anxiety (Chairat, 2015; Ngo et al., 2017); thus, lecturers' L1 can help lower the students' affective filter and encourage them to better engage in L2, as some lecturers in this study did. In this light, EFL non-major students can benefit when the L1 is included in their lesson: L1 both accommodates their L2 comprehension and reduces their L2 anxiety (Bukhari \& Aziz, 2020; Jawhar, 2018), which also has a positive impact on their overall L2 learning.

However, although L1 can be useful in some situations, teachers' excessive use of L1 can reduce learners' L2 awareness and richness of the L2 learning environment (Savran Celik \& Aydin, 2018). Such overuse of L1 can demotivate EFL students because they do not have many opportunities to have contact with English outside classrooms (Tsukamoto, 2012). This is further strengthened by the findings of this study which demonstrate that English should be used predominantly over Vietnamese in all cases. Firstly, the communicative language teaching (CLT) approach has been applied in most of the tertiary EFL classrooms in Vietnam; and the two research sites were making an effort to adopt CLT as a guiding teaching method. CLT advocates that learners should be presented with authentic listening materials and engaged in 
effective communication in meaningful real-life contexts in the target language. Secondly, most of the lecturers expressed the view that it was important to minimise the amount of L1 use and increase L2 use in the classroom. Similarly, students expected their lecturers to predominantly use L2. In this line, from both the lecturers' and students' perspectives and principles of CLT, English should be a dominant language of EFL classroom teaching and communication.

It is also implied from the findings that English could have been used more than it was, if lecturers had a greater awareness of their L2 use and students made more of an effort. This study found many instances where lecturers used L1 out of habit and when some students had the ability to respond in L2 but were too shy to or did not want to. This suggests that the amount of L1 can be reduced, giving way to more L2 use in class if lecturers are more aware and cautious with their L1 use; and students are more confident or had a more positive attitude towards using L2. Furthermore, there are some other ways, as recommended by some student participants, that lecturers can make their L2 more comprehensible using L2 (e.g. simplification) instead of using the L1. This implicitly states that a better consciousness and skills in using L2 communication strategies to make their L2 speech more comprehensible and accessible to students and encourage students' responses in L2 can help maximising L2 use in EFL classrooms.

Based on these points, it is suggested that the overall amount of L2 should generally be more than L1 in EFL classes under all circumstances, although the question of how much L1 and L2 are used "can only be answered by careful consideration of that context by the teacher" (Kerr, 2019, p. 19).

There could be some benefits when an English-dominant policy is implemented in all EFL contexts. Currently, as there have not been any official policies on English medium instruction in Vietnamese EFL teaching, lecturers can decide for themselves how much English is used in their classes (see 3.3.2). This may lead to significant differences in the proportion of L1 and L2 use across lecturers, and across the many teaching contexts around the country. Thus, if an official English-dominant policy was to be ratified, it could be a useful and empowering first step for EFL lecturers and students to become more aware of their language use, which could help minimise the dominant or subconscious L1 use and maximise L2 input and output opportunities for students. Also, if lecturers were directed to an expected higher L2 use, it is likely that they would actively plan their language use and try the use of different strategies to make their L2 more comprehensible and accessible to their students instead of resorting to L1 
straight away. This would be beneficial to both lecturers and students since using a lot of L2 is perceived by lecturers to be one way to enhance their L2 proficiency, and by students to give them more L2 exposure.

Overall, instead of empowering institutions and lecturers to decide for themselves whether to adopt English medium instruction or not, it is advisable that the Vietnamese Education Ministry should ratify an English-dominant approach in all EFL classes.

\subsection{EFL Syllabus and Assessment for Non-English Major Students}

This study has identified that the lesson types and lecturers' skills significantly decided their classroom language choice and the L1/L2 amount (see 4.3.4). Furthermore, how English was assessed during the course influenced both teaching and learning behaviours (see 4.4.5). Along with the findings on the students' and lecturers' perceptions of their EFL classroom, this study has some implications for the design of EFL syllabus and assessment so that they could better facilitate CLT and improve overall learning outcomes in tertiary non-English major classes.

One of the significant findings of this study was that many of the students had a strong desire and expectation for their English learning to be constantly related to their future job. Specifically, students said they were more motivated to engage with their lecturers' English speaking if it contained the content that is relevant to their majors or potential careers. The content of lecturers' speech is normally based on the teaching materials, which implies that the content of textbooks needs to be career-focused as well. In other studies, non-English major students were strongly motivated by wanting to prepare for future career activities (Chairat, 2015; Lobo \& Gurney, 2014). Although the English curriculum includes English for future jobs in the last year of their training programme, it seems to be insufficient and unresponsive to the reported needs of the students. Thus, it is recommended that all EFL syllabi need to integrate job-related content and relevant functional language uses which are relevant to their future careers and work situations. For instance, students of information technology (IT) need to have knowledge of both IT-related vocabulary and how to start a conversation with IT service customers. Such kinds of job-related knowledge and practical communication skills are thus recommended to be a new orientation for a more radical syllabus and curriculum innovation in most EFL tertiary teaching contexts.

Two issues regarding the current English assessment and examinations also emerged from the data. Firstly, students in both schools were evaluated via two written tests (one mid-term and one final term), and one speaking test which on average lasted 5-10 minutes (see 4.4.5). With 
the current writing-dominant and form-based assessment system, students tend to believe that they have to be proficient in grammar and do not need to be good at speaking (Coskun, 2016). This mindset among EFL learners was also found in this study. The traditional writing-oriented language-testing system may have resulted in most Vietnamese tertiary students being qualified for graduation, whilst they cannot communicate successfully with foreigners or meet the basic requirements of work (Nguyen, 2016; Vu, 2017). This shows that the writing-oriented assessment has been hindering the communicative learning and teaching approach. Also, while recent efforts have been made to incorporate speaking tests in the English curriculum, those assessments are insufficient and lack a close link to students' future work contexts. From the students' perspectives, one speaking test at the end of the course on irrelevant topics fails to really motivate and encourage their regular and more active engagement with spoken English during and between classes. The frequency and content of assessments seem to be the most powerful driving forces to students' learning, which might be because two of the strongest motives of non-major students for learning English are to graduate and to be able to use English for their future career (Ngo et al., 2017; Shin et al., 2016).

Hence, two changes are recommended in the way English is assessed. Firstly, there should be an increase in the frequency of oral assessment. Instead of one end-of-term test, monthly tests are more likely to encourage both lecturers and students to take more advantage of classroom oral discourse opportunities. Since the learning motivation of non-English majors is mainly to obtain satisfactory scores for passing the credit and the exams (see 4.3.1), more frequent testing on the speaking skill can make students more aware of having more opportunities to talk and engaging in regular conversations in English. Secondly, the themes and topics of the speaking tests needs to be more interesting and closer to students' training major and future jobs so that they can see the need for the assessment tests and thus have more positive attitudes toward those tests.

It is important for there to be specifically designed syllabi and assessments for each group of students, based on their training majors. Textbooks, teaching/learning resources, and tests are suggested to be more job-oriented and be updated frequently to better meet students' needs and more importantly prepare tertiary students for the language requirements of the job market. These findings regarding the need to redesign the English syllabus and to reformat the testing system at tertiary EFL teaching are two issues that are also relevant for the Vietnamese National Foreign Languages Project 2020. 


\subsection{EFL Teacher Self-reflection and Students' Feedback}

Findings have shown that, despite a certain level of teacher language awareness, there were still mismatches between the lecturers' own perceptions and practices in using L1 and the uses of certain CSs (see chapter 5 \& section 6.3). This study also highlights that lecturers' classroom practices did not match students' expectations. Hence, it is suggested that lecturers need two major tools which can help them observe, evaluate, and be better aware of the way they behave in their EFL classrooms, and therefore modify their practices to better match their students' needs. Those tools are self-reflection and student feedback.

Self-reflection can be a powerful tool for lecturers to create awareness of their teaching strategies, particularly when "so much of what and how they teach can change in the moment" (Widya et al., 2020, p. 2). In fact, the cultural features of each class and the characteristics of its students are always different, which creates tensions and unexpected situations facing lecturers. This is evident in this study when most lecturers overestimated how much L2 they actually used in classes, or when they had a different perception of how their use of simplification techniques differed from how they actually used them. These discrepancies indicate that lecturers' real practices vary from class to class and lesson to lesson, and that lecturers are not necessarily aware of these differences. Thus, lecturers "must engage in critical reflection ... in order to support improved learning outcomes" and to "enhance their capacity to make sense of challenging issues and to make meaning in complex circumstances" (Morgan, 2017, pp. 42, 46). By acknowledging the actualisation of their practice, lecturers can make relevant adjustments and useful adaptions for future practices including creating more meaningful lessons and curriculum and making wiser pedagogical decisions.

Self-video review and self-reflection narratives are suggested to be more effective ways of selfreflection than peer observation, at least in the case of Vietnamese tertiary lecturers. During peer observation, a teacher has to focus on completing the lesson plans and may feel nervous and uncomfortable, restricting their capacity to teach in their usual and normal way (Motallebzadeh et al., 2017). The presence of other people can make Vietnamese teachers behave differently, unable to perform planned tasks as they wish, and feel more serious about losing face (Pham, 2014). Thus, results of peer observation may influence the lecturer's face, identity, and professional development opportunity in the hierarchical teacher evaluation system in Vietnam (e.g. from teacher to the head of teacher group, to the Dean of faculty, and then to the principal). Consequently, self-video review may provide a stress-free environment that may represent authentic and real-life practices and produce high-quality data which 
teachers can use to interpret their own performances (Scaffidi et al., 2019). This is particularly essential when lecturers need to consider aspects that cannot be fully reflected in the written form such as their nonverbal behaviours or students' reactions when lecturers are not looking at them. Reviews of written narratives can enable lecturers to bring their classroom experiences to "the level of conscious awareness" and link "their everyday understandings of teaching to the more expert understandings as well as [to] systematically examine practice" (Arshavskaya, 2017, p. 17). Hence, a combination of video and narrative reviews is recommended as an effective means for Vietnamese EFL lecturers to improve their efficiency of classroom teaching.

However, it seems to be insufficient when lecturers reflect on their classroom practice and own cognitive process without taking student feedback as part of their reflection into consideration. It is critical to also identify students' priorities, different characteristics, and learning styles (Sammaknejad \& Marzban, 2016). Findings have shown that a lack of understanding about students' needs was perceived by the lecturers to affect their pedagogical use of English communication and was perceived by the students to influence their willingness to listen, comprehension level, and learning motivation. These were caused by a lack of communication channels between the lecturers and students both inside and outside classroom borders, such as individual conversations, classroom surveys, informal e-mail, online forums, etc. Via such discussion and conversations, lecturers and students can gain a better understanding of each other, which may not only enhance a positive relationship but also help lecturers to have more responsive practices. Hence, getting students' feedback on teaching is an essential element of the efforts that should be made to enhance the quality of teaching and learning processes in tertiary institutions (Al Kuwaiti et al., 2020).

Analysis of student feedback can be a valuable and supportive self-reflection tool which encourages lecturers to frequently reflect upon and evaluate how they perform in the classrooms. This engagement in reflection can help them to see the progress of their teaching from their own and their students' points of view, in order to figure out what should be changed or adapted for better teaching performances. Particularly, self-video review, self-reflection narratives, and student feedback can contribute to Vietnamese EFL lecturers' more effective use of L1 and L2, and a more conscious application of CSs in the classroom. It is also recommended that self-reflection and analysis of student feedback should be undertaken at regular intervals during each course, to enable lecturers to have a systematic evaluation and 
comparison, allow for timely adjustments to be made and to promptly respond to students' needs.

\subsection{EFL Teacher Education and Competence Standardisation}

The findings of this research have also highlighted the impact of EFL lecturers' professional education and language proficiency on their teaching and students' learning. Hence, my final discussion of implications and recommendations relate to these two aspects in the Vietnamese EFL teaching context.

To begin, this study's results imply that lecturers' knowledge from their previous teacher education programmes influences their understanding of classroom language and communication strategy uses. However, due to different constraints and assumed perceptions about their students' learning motivation and purposes, the lecturers' classroom practices did not reflect many ideas espoused from the knowledge or cognition they acquired from teacher education. Instead, all the lecturers in this study oriented their understandings of L1 and CSs use more towards an everyday perspective that suited the teaching settings. Hence, it is suggested that education programmes for EFL teachers move beyond the provision of content knowledge and general theories in teaching methodology and also include pedagogical techniques and skills. Those programmes need to provide prospective teachers with more practical experience in how to teach English communicatively and effectively in actual classroom contexts. Currently, the duration of the teaching practicum often lasts between four and eight weeks in local schools (Vietnamese Government, 2019). It appears that this may not be enough time for student teachers to develop a comparison between their initial perceptions and the theories in language teaching and to become initially aware of the complexities of classroom practices. Hence, it is recommended that pre-service teacher education curriculum be revised to include more practicums and field experience programs for student teachers.

This study has provided evidence for the potential influence of lecturers' CSs use on the development of students' L2 learning. To date, the importance of using CSs when speaking English have been overlooked in the training syllabus for pre-service EFL teachers in Vietnam. The curriculum designed for English major students is divided into two stages: (1) General English focused on developing their proficiency; and (2) theoretical subjects such as methodology, translation, pragmatics, and others to develop their subject knowledge and theory in teaching (Nguyen, 2017). In fact, in the most updated curriculum for pre-service EFL teachers, there is one credit for communication science; however, it is optional and introduces 
general concepts of communication, written and taught in Vietnamese (Dong Thap University, 2019). Hence, this credit is only able to equip students (a limited number) with general theories, but it does not teach them how to apply these in classroom L2 communication. Obviously, classroom English communication takes up a large proportion of time and effective L2 communication is a target in EFL classrooms. This study strongly suggests that English communication strategies and practical communication techniques in EFL classes should be regarded as compulsory parts of teacher education programmes. When teachers are fully aware of their language use and have systematic knowledge and discourse skills in L2 communication, they can use CSs strategically to enhance their students' learning.

It is recommended that professional training for language teachers should also be more concerned with how teachers can use classroom communication more effectively. As evidenced in the findings, lecturers can strengthen the affective elements of classroom teaching via such strategies as purposive L1, sense of humour, interactional, and nonverbal strategies. Therefore, language teachers' professional education should include training techniques for demonstrating empathy, concern, and emotional support for students' language learning needs. More importantly, teacher awareness is crucial to effective language teaching and learning. The more aware an L2 teacher is, the greater the quality of L2 learning that can be achieved. Hence, it is suggested that EFL teachers be required to achieve highly critical teacher language awareness in order to more consciously use communication strategies in handling communication errors/breakdowns, promoting teacher-student interaction, and enhancing students' English learning.

Findings have demonstrated that the lecturers' perceptions and pedagogical practices are mostly cognisant of their students (see 6.2.2). Thus, pedagogical learner knowledge (see 2.3.1) can allow L2 teachers to decide what optimal conditions for L2 use that particular learner group needs, as well as what cognitive processing capacities can be activated for L2 to emerge (Le, 2020). Language teaching is influenced and shaped by the social and cultural setting in which it takes place including student traits, classroom culture, and institutional or societal factors (Li, 2020; Vygotsky, 1997). In addition to recommendations regarding new EFL teaching directions including a greater awareness of students' expectations and motivations, this thesis also suggests contextual, affective, and pedagogical learner knowledge should be part of critical language teacher awareness and the teacher knowledge base.

In this study, students voiced the opinion that lecturers' English proficiency, reflected via a native-like speaking style, were good models for them to imitate and learn fluent and natural 
pronunciation. The lecturers were also aware of the need to maintain their proficiency; and it seemed to them that trying to use a lot of English in their teaching practices was a good and effective way to achieve this. However, it can be challenging for teachers to further improve their English competence once they have started their teaching and settled down to family life. Hence, regulating competence standardisation in pre-service English language teacher education and encouraging on-going proficiency maintenance for in-service English language teachers may be effective steps. Recently, there has been an official government regulation that all the graduates from English teacher training programmes are expected to have good English competence of at least CEFR levels 4 and 5 by the year 2025 (B2 and C1) (Vietnamese Government, 2017). However, it is suggested that the standard international Common European Framework of Reference for Languages is adopted for prospective teachers instead of a Vietnamese version of CEFR. A standardised framework can help accurately measure future teachers' language competence, which may boost their confidence in their L2 skills during practical teaching. Meanwhile, institutions and authorities need to consider providing in-service EFL teachers with support to refresh their English competence, such as funding or giving them time to retake the test on a regular basis, to help them maintain their general English skills.

However, the general English proficiency is insufficient to ensure teachers' effective use of English to teach English in different classroom contexts. Findings of this study on many mismatches between the lecturers' practices and students' perceptions regarding to communication strategy use and the classroom language choice imply that EFL teachers also need further and regular training on classroom English proficiency. In Vietnamese EFL teaching contexts where the numbers of qualified foreign language teachers and proficiency of local EFL teachers are limited, training on classroom English skills for in-service teachers "can be considered as a strategic choice and hence, should be prioritised" (Pham, 2018, p. 339). Such classroom English proficiency training is significant in raising teachers' awareness of the role and features of classroom English. Thus, this study recommends training on classroom English proficiency be a part of both pre- and in-service EFL teachers since this training can help those teachers change the ways they use classroom language to bring more positive changes in students' behaviours and create better L2 input for students' SLA.

In summary, it is recommended that EFL teacher education programmes increase the pedagogical knowledge and skills content and incorporate more practical field experience programs. It is important to acknowledge and address teachers' higher critical language 
awareness (under the sociocultural and affective domains), pedagogical learner knowledge, and English communication strategies during those training programmes. Measurement to assess teacher competence should be standardised based on an international reference framework. Reaching a certain level on this internationally acknowledged language test should become a compulsory requirement for pre-service English language teacher education, and support needs to be given for in-service English language teachers to maintain their attained proficiency level through the establishment of appropriate policies. Equally important, classroom English proficiency training should also be prioritised for both pre-service and in-service teacher education and professional development programmes.

\subsection{Challenges and Limitations of the Thesis}

The data collection process for this thesis was not without its challenges as some of the initial plans for the data collection field trip had to be changed on the spot due to unforeseen circumstances. First of all, although the lecturers had approved (via email) the use of video recording for the observations, they all changed their minds without giving reasons when I met them at the sites in the first week. As a result, I was worried that I would not be able to capture all their behaviours with audio-recording only. Fortunately, the pilot studies were all conducted without cameras (see section 3.8). This meant that I had developed my fieldnote writing skills and thus had practice in noticing and writing down as many observable behaviours as possible during the official observations. It is possible that the audio-recording and note-taking data might have missed some nonverbal behaviours of the lecturers. Also, one lecturer and some students withdrew their participation at the last minute, which could have limited the quantity and quality of information I aimed to reach. However, with careful preparation for the interview questions and the use of probing follow-up, I consider that I was able to collect sufficient data which have provided rich insights into the research phenomenon.

There are also some other potential limitations of the research at a macro level. There were only five lecturers and 257 students from two colleges included in this study. Firstly, investigating the five college lecturers might also overlook a possibility that university lecturers' practices can be significantly different and worth researching. Similarly, this thesis only demonstrated the classroom English used by lecturers of non-English major students, missing the opportunity to compare major and non-major lecturers and students. Although the two groups of lecturers share many characteristics, their students are not identical in learning motivation and English proficiency; thus, focusing on the non-major group could miss out the influence of English major students' motivation and proficiency variables on lecturers' use of 
CSs in the classroom. Secondly, only 21 of the 257 students joined the focus group interviews, which could have restricted the richness of interview data. However, despite those limitations, this study has contributed to studies of EFL contexts by depicting the complex correlation between the lecturers' and students' perceptions, the lecturers' practices and their own perspectives, and the lecturers' practices and students' expectations. This study is unique in researching overall aspects of classroom communication at non-English major tertiary level in the Vietnamese EFL context.

\subsection{Directions for Future Studies}

Based on the literature review, research findings, and analysis, it is clear that the use of communication strategies, as an integral aspect of classroom oral discourse, deserves more attention from researchers.

Firstly, it is suggested that there should be a broader framework for exploring how conditions such as affective, contextual, cognitive, pedagogical, and sociocultural disciplines interact, influence, and accommodate the use of CSs in EFL classroom oral discourses. This study has described the specific purposes and characteristics of CSs used by lecturers speaking English to students with limited proficiency and low motivation. The analysis has identified some aspects of CSs use that have previously been overlooked such as the origins of the communication breakdown, the influence of interlocutors' characteristics (e.g. language proficiency), and other objective factors (e.g. power effects) influencing the use of CSs. Although communication breakdowns prompt CSs and the final goal of CSs is to reach specific communicative goals, the above underlying conditions which decide how CSs can be effectively used are also important. This could be a new CSs research orientation for other communication contexts and situations.

Secondly, this study has also shown the complexities and different sources of teachers' perceptions and awareness of CSs at the micro level; these could be further studied at the ontogenetic level in a longitudinal study. Moreover, little is known to date about whether lecturers of major and non-major students differ in their use of CSs, which could be a research topic within a large case investigation. Findings from those studies could yield more valuable insights that contribute to the current CSs literature.

Finally, four interesting topics for future studies in the CSs field with a focus on learners have emerged from this study. They are: 1) the correlation between learners' L1 CSs and L2 CSs; 2) learners' cognition and beliefs about their CSs; 3 ) the impact of different factors (e.g. power 
effects, relationships ...) on learners' CSs; and 4) learners' CSs in other communication discourses (e.g. social texts, communicating with foreigners). Some of the above directions have already been studied in different educational and cultural contexts. However, there is little CSs research in Vietnamese EFL contexts, whose identical characteristics are assumed to have different influences on learners' behaviours and cognitions. Thus, I believe that future CSs studies in the four orientations could contribute to enhancing the quality of teaching and learning for Vietnamese EFL courses.

\section{Conclusion}

This study has revealed three key aspects of classroom English communication as used by Vietnamese EFL lecturers teaching non-English major students in two public colleges in Vietnam. Firstly, this research has explored the balance between Vietnamese and English in lecturers' classroom talk. Secondly, it has revealed seven main types and five basic functions of English communication strategies the lecturers used. Thirdly, it has examined the perceptions of both the lecturers and students relating to different aspects of classroom communication, such as affordances and barriers to using or comprehending English communication. The complexities around lecturers' practices and perceptions regarding the use of classroom English communication were discussed in relation to language teacher awareness and knowledge base as well as students' perceptions. Teachers' practices of classroom communication strategies were strongly influenced by the user and analyst domain of their language awareness, while their perceptions regarding communication strategies were mainly shaped by the teacher domain and knowledge about learners. This study found that affective, contextual, and cultural factors played key roles in the lecturers' pedagogical decisions and practices regarding language choice and in the students' comprehension of lecturers' English and their learning motivation. Hence, findings from this research have enabled construction of a new communication strategy taxonomy with its functions and characteristics being different from previously established typologies. Additionally, tensions and mismatches between how lecturers see classroom English communication and how students perceive what works for them were identified. The main reasons for the perceptual gaps were a lack of understanding and connection at the interpersonal level, possibly caused by the hierarchy of teacher-student relationships. This study has suggested some solutions to reduce this gap.

In conclusion, my thesis has established that lecturers' classroom English communication is a crucial component of EFL teaching. Particularly, lecturers' communication styles and 
strategies are influential in promoting students' learning skills and initiating and maintaining a positive relationship with their students. Lecturers' English communication strategies and behaviours, together with a positive lecturer-student relationship, supportive learning environment, and the provision of plentiful interaction opportunities in EFL classes can enable students to have a stronger sense of belonging to the class community, reduce their L2 anxiety, and feel more motivated in their EFL learning. This is particularly important for non-English major students whose L2 proficiency and learning motivation is normally low. Meanwhile, for lecturers' classroom communication to be more effective, a higher level of critical language awareness, contextual and discourse knowledge, and both general and classroom English proficiency should be combined with a sufficient understanding of students' needs and expectations. In other words, pedagogical learner knowledge and co-constructed teaching in which lecturers learn from their students so that their teaching will better meet students' needs is pivotal for a learner-centred teaching approach.

Through conducting this study, I have learnt about the significance and complexities of classroom communication and the influential roles of different factors especially that of learners on lecturers' classroom language choice and English communication strategies. I hope that my study will help other EFL teachers, particularly in the Vietnamese non-English major teaching context, to have a better understanding of classroom English communication so that they can use them strategically to foster their students' English learning. 


\section{References}

Abdelati, S. (2019). Communication strategies in Libyan EFL classrooms: Materials, perceptions and practices [Doctoral dissertation, Sheffield Hallam University]. http://shura.shu.ac.uk/25492/1/Abdelati_2019_PhD_CommunicationStrategiesIn.pdf

Abu-Shanab, E., \& Nor, K. (2013). The influence of language on research results. Management Research and Practice Journal (MRP), 4(2), 37-48.

Adamou, V. (2017). English language teachers' perceptions and challenges in adopting the communicative language teaching (CLT) approach [Bachelor's paper, University of Greenwich]. https://www.researchgate.net/publication/321383578_English_Language_Teachers'_P erceptions_and_Challenges_in_Adopting the_Communicative_Language_Teaching CLT_Approach

Adams, A., \& Cox, A. L. (2008). Questionnaires, in-depth interviews and focus groups. Cambridge University Press. https://doi.org/10.1017/CBO9780511814570.003

Afrin, S. (2016). Writing problems of non-English major undergraduate students in Bangladesh: An observation. Open Journal of Social Sciences, 4(3), 104-115.

Agezo, C. (2010). Why teachers leave teaching: The case of pretertiary institutions in Ghana. International Journal of Educational Reform, 19(1), 51-69. https://doi.org/10.1177/105678791001900104

Aguilar, M. (2017). Engineering lecturers' views on CLIL and EMI. International Journal of Bilingual Education and Bilingualism, 20(6), 722-735.

Agyekum, S. (2019). Teacher-student relationships: The impact on high school students. Journal of Education and Practice, 10(14), 121-122. https://doi.org/10.7176/JEP

Akbari, R., \& Dadvand, B. (2014). Pedagogical knowledge base: A conceptual framework for teacher admission. System, 42, 12-22. https://doi.org/10.1016/j.system.2013.11.001

Akhter, N. (2018). Role of English-Bangla code-switching in vocabulary retention: A case study at university of Dhaka. In R. Chowdhury, M. Sarkar, F. Mojumder, \& M. M. Roshid (Eds.), Engaging in educational research: Revisiting policy and practice in Bangladesh (Vol. 44, pp. 299-314). Springer. https://doi.org/10.1007/978-981-130708-9_16

Albahri, M., Yang, P., \& Moustakim, M. (2018). Saudi Arabian tertiary teachers' and learners' attitude towards the communicative language teaching (CLT). International Journal of Information and Education Technology, 8(4), 317-321. https://doi.org/10.18178/ijiet.2018.8.4.1055

Al-Amer, R., Ramjan, L., Glew, P., Darwish, M., \& Salamonson, Y. (2015). Language translation challenges with Arabic speakers participating in qualitative research 
studies. International Journal of Nursing Studies, 54(2016), 150-157.

https://doi.org/10.1016/j.ijnurstu.2015.04.010

Al-Garni, S. A., \& Almuhammadi, A. H. (2019). The effect of using communicative language teaching activities on EFL students' speaking skills at the university of Jeddah. English Language Teaching, 12(6), 72-86. https://doi.org/10.5539/elt.v12n6p72

Al-Ghafri, B., Mirza, C., \& Gabarre, S. (2019). EFL teachers' use of learners' L1 in cycle two schools in Oman. Asian Research Journal of Arts \& Social Sciences, 8(4), 1-10. https://doi.org/10.9734/arjass/2019/v8i430107

Al-Ghamdi, H., \& Al-Bargi, A. (2017). The impact of teacher speech modification on the quality of interaction and learning: An analysis of spoken discourse in Saudi EFL classrooms. International Journal of Linguistics, 9(3), 79-101. https://doi.org/10.5296/ijl.v9i3.11382

Al Kuwaiti, A., Subbarayalu, A. V., \& Ramzi, O. I. (2020). Gender differences in medical students' perception of lecturing skills. Majmaah Journal of Health Sciences, 8(2), $51-65$.

Al Sharaeai, W. A. A. (2012). Students' perspectives on the use of L1 in English classrooms. (Publication Number 12898) [Master's thesis, Lowa State University]. https://lib.dr.iastate.edu/etd/12898/

Alhodiry, A. A. (2016). The Libyan EFL teachers' role in developing students' motivation. Procedia-Social and Behavioral Sciences, 232(2016), 83-89. https://doi.org/10.1016/j.sbspro.2016.10.020

Alijani, A., \& Barjesteh, H. (2018). The impact of using L1 for teaching grammar among Iranian EFL learners. English Literature and Language Review, 4(3), 26-31.

Almoayidi, K. (2018). The effectiveness of using L1 in second language classrooms: A controversial issue. Theory and Practice in Language Studies, 8(4), 375-379. https://doi.org/10.17507/tpls.0804.02

Alshenqeeti, H. (2014). Interviewing as a data collection method: A critical review. English Linguistics Research, 3(1), 39-45. https://doi.org/10.5430/elr.v3n1p39

Alsolmi, A. (2017). Teachers of students with visual disability: Their perceptions and knowledge of assistive technology in saudi arabia (Publication Number 2016912384) [Doctoral thesis, Saint Louis University]. ProQuest One Academic.

Altalhab, S. (2018). Short- and long-term effects of repetition strategies on vocabulary retention. Advances in Language and Literary Studies, 9(2), 146-149. https://doi.org/10.7575/aiac.alls.v.9n.2p.146

Alwazir, B., \& Shukri, N. (2017). The use of CLT in the Arab context: A critical perspective. International Journal of English Language Education, 5(1), 15-32. https://doi.org/10.5296/ijele.v5i1.10486

Aly, E. H. A. Y. (2020). Learners and teachers' attitudes toward using L1 in Arabic classes: Does context matter?. European Journal of Foreign Language Teaching, 4(4), 1-20. https://doi.org/10.5281/zenodo.3691935 
Anbesie, T. (2020). The distinction between knowledge base of teacher education and ELT education. IOSR Journal of Humanities and Social Science, 25(1), 46-52. https://doi.org/10.9790/0837-2501034652

Andarab, M. S., \& Mutlu, A. K. (2019). Using humor in language classrooms: Greasing the wheels or putting a spanner in the works? A study on humor styles of Turkish EFL instructors. Eurasian Journal of Applied Linguistics, 5(1), 23-39.

Anderson, C. (2010). Presenting and evaluating qualitative research. American Journal of Pharmaceutical Education, 74(8), 1-7. https://doi.org/10.5688/aj7408141

Andrews, S. (2001). The language awareness of the L2 teacher: Its impact upon pedagogical practice. Language Awareness, 10(2-3), 75-90.

Andrews, S. (2007). Teacher language awareness. Cambridge University Press.

Andrews, S., \& Lin, A. M. (2017). Language awareness and teacher development. In J. M. C. Peter Garrett ( $1^{\text {st }}$ ed.), The Routledge handbook of language awareness (pp. 57-74). Routledge. https://doi.org/10.4324/9781315676494

Andrews, S., \& Svalberg, A. M. (2017). Teacher Language Awareness. In J. Cenoz, D. Gorter, \& S. May (3rd ed.), Language Awareness and Multilingualism (pp. 219-231). Springer International Publishing. https://doi.org/10.1007/978-3-319-02240-6_17

Andrews, S. J. (1997). Metalinguistic awareness and teacher explanation. Language Awareness, 6(2-3), 147-161. https://doi.org/10.1080/09658416.1997.9959924

Angelovska, T. (2017). Beyond instructed L2 grammar acquisition: Theoretical insights and pedagogical considerations about the role of prior language knowledge. Studies in Second Language Learning and Teaching, 7(3), 397-417.

Anggrahini, N. W. (2019). Students' perception on the use of L1 in the English language classroom in SMA Taman Siswa Mojokerto. RETAIN, 7(1), 139-146.

Ansori, M., Nurkamto, J., \& Suparno. (2019). Teacher's beliefs and practices in the integration of higher order thinking skills in teaching reading. ELS Journal on Interdisciplinary Studies on Humanities, 2(4), 541-555. https://doi.org/10.34050/elsjish.v2i4

Antón, M., \& DiCamilla, F. (1998). Socio-cognitive functions of L1 collaborative interaction in the L2 classroom. Canadian Modern Language Review, 54(3), 314-342.

Ariatna, A. (2016). The need for maintaining CLT in Indonesia. TESOL Journal, 7(4), 800 822. https://doi.org/10.1002/tesj.246

Armstrong, J. M., Haskett, M. E., \& Hawkins, A. L. (2017). The student-teacher relationship quality of abused children. Psychology in The Schools, 54(2), 142-151. https://doi.org/10.1002/pits.21989

Arshad, Z., Abdolrahimpour, M., \& Najafi, M. R. (2015). The use of L1 as a consciousnessraising tool in teaching grammar to beginner and upper-intermediate EFL students. Journal of Language Teaching and Research, 6(3), 633-638. https://doi.org/10.17507/jltr.0603.21 
Arshavskaya, E. (2017). Becoming a language teacher: Exploring the transformative potential of blogs. System, 69, 15-25.

https://doi.org/https://doi.org/10.1016/j.system.2017.08.006

Athawadi, A. (2019). Factors hindering the implementation of communicative language teaching in Libyan university English classes. European Journal of English Language Teaching, 4(3), 60-74. https://doi.org/10.5281/zenodo.256400

Atkins, L., \& Wallace, S. (2012). Qualitative research in education. Sage. https://dx.doi.org/10.4135/9781473957602

Aulia, M. (2016, November 12-13). Teacher language awareness and communication strategies in Syiah Kuala university, Aceh, Indonesia [Conference session]. Proceedings of English Education International Conference, Banda Aceh, Indonesia. https://scholar.google.com/scholar?hl=en\&as_sdt=0\%2C5\&q=Teacher\%E2\%80\%99s +beliefs+and+practices+in+the+integration+of+higher+order+thinking+skills+in+tea ching+reading \&btnG $=$

Awaiko Westin, C. I. (2019). Student motivation in L2 English teaching and learning: A study on students' perspectives of L2 classroom-centered motivational practices at a lower-secondary school [Bachelor's paper, Stockholm University]. http://urn.kb.se/resolve?urn=urn:nbn:se:su:diva-179649

Awada, M. R., Mubarakb, L. A., \& Salehc, M. S. (2020). The effect of using L1 in EFL classrooms: The case of Iraqi EFL primary school learners. International Journal of Innovation, Creativity and Change, 12(7), 283-292.

Ayaz, A. (2017). The effects of teachers' code Switching on EFL learners' speaking skill. International Journal of Language Academy, 5(5), 59-66.

Aydin, S. (2012). Factors causing demotivation in EFL teaching process: A case study. Qualitative Report, 17(101), 1-13.

Badash, M., Harel, E., Carmel, R., \& Waldman, T. (2020). Beliefs versus declared practices of English as a foreign language (EFL) teachers regarding teaching grammar. World Journal of English Language, 10(1), 49-61. https://doi.org/10.5430/wjel.v10n1p49

Baioumy, N., Ismail, Z., Ab Rahman, R., Annuar, T., Mohamad, Z., \& Haridi, E. (2018). The effectiveness of a proposed strategy based on video stories on Youtube in the development of listening and speaking skills among the non-Arabic speakers. International Journal of Academic Research in Business and Social Sciences, 8(10), 414-436. https://doi.org/10.6007/IJARBSS/v8-i10/4745

Baker, A. A. (2011, September 18). ESL teachers and pronunciation pedagogy: Exploring the development of teachers' cognitions and classroom practices [Paper presentation]. The 2nd Pronunciation in Second Language Learning and Teaching Conference, Ames, IA.

Ballester, E. P. (2015). Verbal and nonverbal teacher immediacy and Foreign Language anxiety in an EFL university course. Porta Linguarum, 23, 9-24.

Barglowski, K. (2018). Where, what and whom to study? Principles, guidelines and empirical examples of case selection and sampling in migration research. In R. Zapata-Barrero 
\& E. Yalaz (Eds.), Qualitative research in European Migration studies (pp. 151-168). Springer. https://doi.org/10.1007/978-3-319-76861-8_9

Barnard, R., \& Nguyen, G. (2010). Task-based language teaching (TBLT): A Vietnamese case study using narrative frames to elicit teachers' beliefs. Language Education in Asia, 1(1), 77-86.

Bashiruddin, A. (2013). Reflections on translating qualitative research data: Experiences from Pakistan. International Journal of Applied Linguistics, 23(3), 357-367. https://doi.org/10.1111/ijal.12041

Baumeister, R., \& Leary, M. (1995). The need to belong: Desire for interpersonal attachments as a fundamental human motivation. Psychological Bulletin, 117(3), 497529. https://doi.org/10.1037/0033-2909.117.3.497

Beckett, G., \& Li, F. (2012). Content-based English education in China: Students' experiences and perspectives. Journal of Contemporary Issues in Education, 7(1), 4763 https://doi.org/10.20355/C5B59J

Benali, H. (2013). Teaching oral and written communication strategies to enhance the Spanish EFL learners' fluency and self-confidence [Doctoral dissertation, Universidad de Salamanca].

https://gredos.usal.es/bitstream/handle/10366/121335/DFI_BenaliHanane Tesis.pdf?s equence $=3$

Bennett, N. J. (2016). Using perceptions as evidence to improve conservation and environmental management. Conservation Biology, 30(3), 582-592. https://doi.org/10.1111/cobi.12681

Bernaus, M., \& Gardner, R. (2008). Teacher motivation strategies, student perceptions, student motivation, and English achievement. The Modern Language Journal, 92(3), 387-401. https://doi.org/10.1111/j.1540-4781.2008.00753.x

Bernaus, M., Wilson-Daily, A., \& Gardner, R. (2009). Teachers' motivation, classroom strategy use, students' motivation and second language achievement. Porta Linguarum, 12(2), 25-36.

Bhooth, A., Azman, H., \& Ismail, K. (2014). The role of the L1 as a scaffolding tool in the EFL reading classroom. Procedia-Social and Behavioral Sciences, 118, 76-84. https://doi.org/10.1016/j.sbspro.2014.02.011

Bialystok, E. (1990). Communication strategies in second language acquisition. Blackwell Publishing.

Bialystok, E. (2001). Bilingualism in development: Language, literacy, and cognition (1st ed.). Cambridge University Press.

Birello, M. (2012). Teacher cognition and language teacher education: Beliefs and practice. A conversation with Simon Borg. Bellaterra Journal of Teaching \& Learning Language \& Literature, 5(2), 88-94. https://doi.org/10.5565/rev/jtl3.464

Blume, C., Gerlach, D., Roters, B., \& Schmidt, T. (2019). ABCs of inclusive teacher education: A quantitative and qualitative study examining the attitudes, beliefs and 
(reflective) competence of pre-service foreign language teachers. TESL-EJ, 22(4), 118.

Bogdan, R. (2007). Qualitative research for education: An introduction to theories and methods (5th ed.). Pearson.

Bongaerts, T., \& Poulisse, N. (1989). Communication strategies in L1 and L2: Same or different? Applied Linguistics, 10(3), 253-268.

Boonkongsaen, N. (2018). Communication strategies used by Thai EFL teachers. The New English Teacher, 12(1), 63-76.

Borg, M. (2001). Key concepts in ELT teachers' beliefs. ELT Journal, 55(2), 186-188. https://doi.org/10.1093/elt/55.2.186

Borg, S. (2003). Teacher cognition in language teaching: A review of research on what language teachers think, know, believe, and do. Language Teaching, 36, 81-109. https://doi.org/10.1017/S0261444803001903

Borg, S. (2011). The impact of in-service teacher education on language teachers' beliefs. Fuel and Energy Abstracts, 39(3), 370-380. https://doi.org/10.1016/j.system.2011.07.009

Bouras, H., \& Keskes, S. (2014, September 8-10). Teacher-learner rapport impact on EFL learners' motivation [Paper presentation]. International Conference on Social Sciences and Humanities, Istanbul, Turkey.

Bozorgian, H., \& Fallahpour, S. (2015). Teachers' and students' amount and purpose of L1 use: English as foreign language (EFL) classrooms in Iran. Iranian Journal of Language Teaching Research, 3(2), 67-81.

Breen, R. L. (2006). A practical guide to focus-group research. Journal of Geography in Higher Education, 30(3), 463-475. https://doi.org/10.1080/03098260600927575

Brevik, L., \& Rindal, U. (2020). Language use in the classroom: Balancing target language exposure with the need for other languages. TESOL Quarterly, O(0), 1-29. https://doi.org/10.1002/tesq.564

Brinkmann, S. (2016). The role of teachers' beliefs in the implementation of learner-centred education in India [Doctoral thesis, UCL Institute of Education, London]. https://discovery.ucl.ac.uk/id/eprint/1532842

Brookfield, S. D., \& Preskill, S. (2012). Discussion as a way of teaching: Tools and techniques for democratic classrooms. John Wiley \& Sons.

Brown, H. D. (2014). Principles of language learning and teaching: A course in second language acquisition (6th ed.). Pearson Education.

Bruen, J., \& Kelly, N. (2014). Using a shared L1 to reduce cognitive overload and anxiety levels in the L2 classroom. The Language Learning Journal, 45(3), 368-381. https://doi.org/10.1080/09571736.2014.908405

Bryman, A. (2006). Integrating quantitative and qualitative research: How is it done? Qualitative Research, 6(1), 97-113. https://doi.org/10.1177/1468794106058877 
Bryman, A. (2012). Social research methods (4th ed.). Oxford University Press.

Bryman, A. (2016). Social research methods (5th ed.). Oxford University Press.

Bucevska, V. (2007). Designing a web versus a paper questionnaire-some general and special issues. Retrieved March 23, 2019 from https://scholar.google.com/scholar?hl=en\&as_sdt=0\%2C5\&q=Designing+a+web+ver

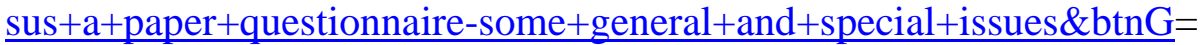

Buendía-Arias, X. P., André-Arenas, A., \& Rosado-Mendinueta, N. d. R. (2020). Factors shaping EFL preservice teachers' identity configuration. Íkala, 25(3), 583-603. https://doi.org/10.17533/udea.ikala.v25n03a02

Buhs, E. S., Ladd, G. W., \& Herald, S. L. (2006). Peer exclusion and victimization: Processes that mediate the relation between peer group rejection and children's classroom engagement and achievement? Journal of Educational Psychology, 98(1), 1-13. https://doi.org/10.1037/0022-0663.98.1.1

Bui, P. H., \& Nguyen, T. T. A. (2014). The use of Vietnamese in English language classes: Benefits and drawbacks. International Journal on Studies in English Language and Literature, 2, 24-26.

Bui, T., Nguyen, T. T. T., \& Nguyen, A. D. (2018). Vietnamese higher education language planning and university students' career development. In J. Albright (Ed.), English Tertiary Education in Vietnam (pp. 54-67). Routledge.

Bui, T. T. N., \& Nguyen, H. T. M. (2016). Standardizing English for educational and socioeconomic betterment-a critical analysis of English language policy reforms in Vietnam. In English language education policy in Asia (pp. 363-388). Springer.

Bui, T. T. Q. (2012). Strategies employed by EFL English majors for coping with communication strategies breakdowns [Doctoral dissertation, Suranaree University of Technology]. http://sutir.sut.ac.th:8080/sutir/bitstream/123456789/4112/2/Fulltext.pdf

Bukhari, F. B., \& Aziz, A. A. (2020). L1 (Bahasa Malaysia) impact in process and product of learning writing in L2 (English). International Journal of Applied Science and Engineering Review, 1(2), 28-58.

Bukve, T. (2020). Students' perspectives on English medium instruction within higher education: Exploring gender differences in Norway and Finland. Apples: Journal of Applied Language Studies, 14(1), 7-24. https://doi.org/10.17011/apples/urn.202002272211

Burch, A. R. (2014). Pursuing information: A conversation analytic perspective on communication strategies. Language Learning, 64(3), 651-684. https://doi.org/10.1111/lang.12064

Burgos, E. G., \& Molina, S. S. (2020). University students' attitudes towards EFL: A case from the South of Chile. Profile Issues in Teachers' Professional Development, 22(1), 43-56. https://doi.org/10.15446/profile.v22n1.75401

Busch, D. (2010). Pre-service teacher beliefs about language learning: The second language acquisition course as an agent for change. Language Teaching Research, 14(3), 318337. https://doi.org/10.1177/1362168810365239 
Butler, Y. G. (2017). Communicative and task-based language teaching in the Asia-pacific region. In N. Van Deusen-Scholl \& S. May (Eds.), Second and foreign language education (pp. 327-338). Springer International Publishing. https://doi.org/10.1007/978-3-319-02246-8_28

Caldwell-Harris, C. L. (2014). Emotionality differences between a native and foreign language: theoretical implications. Frontiers in Psychology, 5(1055), 1-4. https://doi.org/10.3389/fpsyg.2014.01055

Calhoun, A. A. (2019). Teacher-student relationships, classroom environment, and student intrinsic motivation [Doctoral dissertation, University of Findlay]. https://etd.ohiolink.edu/!etd.send_file?accession=findlay1565098233906771\&disposit ion=inline

Çalıklar, Ö., \& Kadığlu, H. (2020). Validity and reliability of the Turkish version of the Healthy Families Parenting Inventory. Journal of Psychiatric Nursing, 11(1), 49-56. https://doi.org/10.14744/phd.2019.93585

Celce-Murcia, M. (2008). Rethinking the role of communicative competence in language teaching. In E. A. Soler \& M. P. S. Jordà (Eds.), Intercultural language use and language learning (pp. 41-57). Springer.

Cemalcilar, Z. (2009). Schools as socialisation contexts: Understanding the impact of school climate factors on students' sense of school belonging. Applied Psychology, 59(2), 243-272. https://doi.org/10.1111/j.1464-0597.2009.00389.x

Chairat, P. (2015). Attitudes and motivation of non-English major students towards learning English as a foreign language: A case study. ASEAN Journal of Education, 1(1), 6983.

Chang, M., \& Goswami, J. S. (2011). Factors affecting the implementation of communicative language teaching in Taiwanese college English classes. English Language Teaching, 4(2), 3-12. https://doi.org/10.5539/elt.v4n2p3

Chapetón Castro, C. M. (2009). The use and functions of discourse markers in EFL classroom interaction. Profile Issues in Teachers Professional Development, 21(1), 57-77. https://doi.org/10.15446/profile.v21n1.76887

Chapple, J. (2015). Teaching in English is not necessarily the teaching of English. International Education Studies, 8(3), 1-13. https://doi.org/10.5539/ies.v8n3p1

Chaudron, C. (1983). Simplification of input: Topic reinstatements and their effects on L2 learners' recognition and recall. TESOL Quarterly, 17(3), 437-458. https://doi.org/10.2307/3586257

Cheung, A., \& Hennebry-Leung, M. (2020). Exploring an ESL teachers' beliefs and practices of teaching literary texts: A case study in Hong Kong. Language Teaching Research, OO(0), 1-26. https://doi.org/10.1177/1362168820933447

Christianto, D. (2019). Teachers' perceptions on the use of the communicative language teaching approach in the English classrooms. International Journal of Indonesian Education and Teaching, 3(1), 90-101. 
Chyung, S. Y., Swanson, I., Roberts, K., \& Hankinson, A. (2018). Evidence-based survey design: The use of continuous rating scales in surveys. Performance Improvement, 57(5), 38-48. https://doi.org/10.1002/pfi.21763

Clark, V. L. P., \& Ivankova, N. V. (2015). Mixed methods research: A guide to the field (Vol. 3). Sage.

Cohen, A. (1998). Strategies in learning and using a second language. Longman.

Cohen, L., Manion, L., \& Morrison, K. (2011). Research methods in education (7th ed.). Routledge.

Cook, V. (2001). Using the first language in the classroom. Canadian Modern Language Review, 57(3), 402-423. https://doi.org/10.3138/cmlr.57.3.402

Coskun, A. (2016). Causes of the "I can understand English but I can't speak" syndrome in Turkey. Journal on English Language Teaching, 6(3), 1-12.

Creswell, J., \& Plano-Clark, V. (2011). The foundations of mixed methods research. In Creswell J \& P.-C. V (Eds.), Designing and conducting mixed methods research. (pp. 19-52). Sage.

Creswell, J., \& Poth, C. (2018). Qualitative inquiry and research design (4th ed.). Sage.

Creswell, J. W. (2009). Research design: Qualitative, quantitative, and mixed methods approaches (3rd ed.). Sage.

Creswell, J. W. (2013). Qualitative inquiry and research design: Choosing among five approaches (3rd ed.). Sage.

Creswell, J. W. (2015). A concise introduction to mixed methods research. Sage.

Creswell, J. W., \& Plano-Clark, V. (2007). Designing and conducting mixed methods research. Sage.

Cross, R. (2010). Language teaching as sociocultural activity: Rethinking language teacher practice. The Modern Language Journal, 94(3), 434-452. https://doi.org/10.1111/j.1540-4781.2010.01058.x

Cuéllar, L., \& Oxford, R. L. (2018). Language teachers' emotions: Emerging from the shadows. In Emotions in Second Language Teaching (pp. 53-72). Springer.

Cuellar, M., \& Johnson-Ahorlu, R. N. (2016). Examining the complexity of the campus racial climate at a Hispanic serving community college. Community College Review, 44(2), 135-152. https://doi.org/10.1177/0091552116632584

Cummins, J., \& Davison, C. (2007). International handbook of English language teaching. Springer. https://doi.org/10.1007/978-0-387-46301-8

Dai, N. T., Free, C., \& Gendron, Y. (2019). Interview-based research in accounting 20002014: Informal norms, translation and vibrancy. Management Accounting Research, 42, 26-38. https://doi.org/10.1016/j.mar.2018.06.002 
Dang, H. T., \& Moskovsky, C. (2019). English-medium instruction in Vietnamese higher education: A ROAD-MAPPING perspective. Issues in Educational Research, 29(4), 1330-1347.

Dang, V. H. (2006). Learner-centeredness and EFL instruction in Vietnam: A case study. International Education Journal, 7(4), 598-610.

Daniel, B. K., \& Harland, T. (2017). Higher education research methodology: A step-by-step guide to the research process. Routledge.

Daniëls, E., Hondeghem, A., \& Heystek, J. (2020). Developing school leaders: Responses of school leaders to group reflective learning. Professional Development in Education, 1-15. https://doi.org/10.1080/19415257.2020.1766543

Darawsheh, W., \& Stanley, M. (2014). Reflexivity in research: Promoting rigour, reliability and validity in qualitative research. International Journal of Therapy and Rehabilitation, 21(12), 560-568.

Davis, H. A. (2001). The quality and impact of relationships between elementary school students and teachers. Contemporary Educational Psychology, 26(4), 431-453. https://doi.org/10.1006/ceps.2000.1068

De la Campa, J., \& Nassaji, H. (2009). The amount, purpose, and reasons for using L1 in L2 classrooms. Foreign Language Annals, 42(4), 742-759. https://doi.org/10.1111/j.1944-9720.2009.01052.x

Dearden, J. (2014). English as a medium of instruction-a growing global phenomenon. British Council. Retrieved April 15 2018, from https://www.britishcouncil.es/sites/default/files/british_council_english_as_a_medium _of_instruction.pdf

Delport, C. S. L., \& Roestenburg, W. J. H. (2011). Quantitative data collection methods: Questionnaires, checklists, structured observation and structured interview schedules. In A. S. In De Vos, Strydom, H., Fouché, C. B., \& Delport, C. S. L. (Ed.), Research at grass roots: For the social sciences and human service professions (4th ed.). Van Schaik Publishers.

Delsi, M., \& Pupung, P. (2019). Teachers' perception related to the implementation of curriculum 2013. Indonesian Journal of Curriculum and Educational Technology Studies, 7(1), 44-52. https://doi.org/10.15294/ijcets.v7i1.27564

Denscombe, M. (2007). The good research guide for small-scale social research projects (3rd ed.). Open University Press.

Denscombe, M. (2014). The good research guide for small-scale social research projects (4th ed.). McGraw-Hill Education.

Denzin, N. K., \& Lincoln, Y. S. (1994). Handbook of qualitative research. Sage.

Dewaele, J.-M., \& Mercer, S. (2018). Variation in ESL/EFL teachers' attitudes towards their students. In S. Mercer \& A. Kostoulas (Eds.), Language teacher psychology (pp. 178195). Multilingual Matters. https://doi.org/https://doi.org/10.21832/9781783099467$\underline{015}$ 
Díaz Larenas, C. (2011). Exploring knowledge of English speaking strategies in 8th and 12th graders. Profile Issues in Teachers' Professional Development, 13(2), 85-98.

DiCamilla, F. J., \& Antón, M. (2004). Private speech: A study of language for thought in the collaborative interaction of language learners. International Journal of Applied Linguistics, 14(1), 36-69.

Dinham, S., \& Scott, C. (2000). Moving into the third, outer domain of teacher satisfaction. Journal of Educational Administration, 38(4), 379-396. https://doi.org/10.1108/09578230010373633

Dippenaar, H., \& Peyper, T. (2011). Language awareness and communication as part of teacher education at the University of Pretoria, South Africa. Journal for Language Teaching, 45(2), 32-45. https://doi.org/10.4314/jlt.v45i2.2

Dixon, L., Zhao, J., Shin, J., Wu, S., Su, J.-H., Burgess-Brigham, R., Unal, M., \& Snow, C. (2012). What we know about second language acquisition. Review of Educational Research, 82(1), 5-60. https://doi.org/10.3102/0034654311433587

Do, K. A. (2013). The current state of the art in the teaching of grammar at Vietnamese high schools. Language in India, 13(3), 22-42.

Dobao, A. M. F. (2001). Communication strategies in the interlanguage of Galician students of English: The influence of learner-and task-related factors. Atlantis, 23(1), 41-62.

Doiz, A., Lasagabaster, D., \& Sierra, J. M. (2012). English-medium instruction at universities: Global challenges. Multilingual matters.

Dörnyei, Z. (1994). Motivation and motivating in the foreign language classroom. The Modern Language Journal, 78(3), 273-284.

Dörnyei, Z. (2001). Motivational strategies in the language classroom. Cambridge University Press.

Dörnyei, Z., \& Scott, M. L. (1997). Communication strategies in a second language: Definitions and taxonomies. Language Learning, 47(1), 173-210. https://doi.org/10.1111/0023-8333.51997005

Drew, C. J., Hardman, M. L., \& Hosp, J. L. (2008). Designing and conducting research in education. Sage.

Durán-Narváez, N. C., Lastra-Ramírez, S. P., \& Morales-Vasco, A. M. (2017). Beliefs of preservice teachers about English language teaching: Reflection and practice. Folios, 45, 177-193.

Dyana, R. (2020). Communication strategies used by the English teacher in aviation class. Manajemen Pendidikan, 15(1), 44-51. https://doi.org/10.23917/mp.v15i1.10745

Ekoç, A. (2020). English medium instruction (EMI) from the perspectives of students at a technical university in Turkey. Journal of Further and Higher Education, 44(2), 231243. https://doi.org/10.1080/0309877X.2018.1527025

Elder, C. (2001). Assessing the language proficiency of teachers: Are there any border control? Language Testing, 18(2), 149-170. 
Ellen, S., \& Taverniers, M. (2011). Advanced EFL learners' beliefs about language learning and teaching: A comparison between grammar, pronunciation, and vocabulary. English Studies, 92(8), 896-922. https://doi.org/10.1080/0013838X.2011.604578

Ellis, R. (1990). Instructed second language acquisition: Learning in the classroom. WileyBlackwell. https://www.semanticscholar.org/paper/Instructed-second-languageacquisition-\%3A-learning-Ellis/fb0cdeb90246b742882d786e1e16d6a1766db4db

Ellis, R. (1994). The study of second language acquisition. Oxford University Press.

Ellis, R. (2004). The definition and measurement of L2 explicit knowledge. Language Learning, 54(2), 227-275. https://doi.org/10.1111/j.1467-9922.2004.00255.x

Ellis, R. (2012). Language teaching research and language pedagogy. Wiley-Blackwell. https://doi.org/10.1002/9781118271643

Ellis, R., \& He, X. (1999). The roles of modified input and output in the incidental acquisition of word meanings. Studies in Second Language Acquisition, 21(2), 285301. https://doi.org/10.1017/S0272263199002077

Epure, M., \& Lorena, C. (2018). The role of language and symbols in promotional strategies and marketing schemes. IGI Global. https://books.google.co.nz/books?id=FjFtDwAAQBAJ

Ewert, A. (2010). An educational language community: External and internal language use by multilingual students. In J. Arabski \& A. Wojtaszek (Eds.), Neurolinguistic and psycholinguistic perspectives on SLA (pp. 159-174). Multilingual Matters. https://doi.org/10.21832/9781847692429-013

Faerch, C., \& Kasper, G. (1983). Plans and strategies in foreign language communication. In C. Faerch \& G. Kasper (Eds.), Strategies in interlanguage communication (pp. 20 60). Longman.

Fang, X. (2010). The role of input and interaction in second language acquisition. Crosscultural Communication, 6(1), 11-17.

Fang, Z. H. (1996). A review of research on teacher beliefs and practices. Educational Research, 38(1), 47-65.

Farrell, T. S., \& Guz, M. (2019). 'If I wanted to survive I had to use it': The power of teacher beliefs on classroom practices. TESL-EJ, 22(4), 1-17.

Farsia, L. (2016). Re-conceptualizing communicative language teaching through sociocultural perspective. Englisia, 3(2), 119-128. https://doi.org/10.22373/ej.v3i2.1137

Fauzi, A. F. A. A., Ali, K. N., \& Amirudin, R. (2019). Evaluating students readiness, expectancy, acceptance and effectiveness of augmented reality based construction technology education. International Journal of Built Environment and Sustainability, 6(1), 7-13. https://doi.org/10.11113/ijbes.v6.n1.309

Feldermann, S., \& Hiebl, M. (2020). Using quotations from non-English interviews in accounting research. Qualitative Research in Accounting \& Management, 17, 229262. https://doi.org/10.1108/QRAM-08-2018-0059 
Ferreira, P. R., Lucena, A. M., Machado-Nascimento, N., Alves, R. O., Alexandre de Souza, V. C., Carvalho, S. A. d. S., \& Camargos Jr., W. (2016). Strategies for perception of mother tongue: From born to one year old. Speech, Language, Hearing Science and Education Journal, 18(4), 982-991. https://doi.org/10.1590/1982-0216201618422715

Feryok, A. (2010). Language teacher cognitions: Complex dynamic systems? System, 38(2), 272-279. https://doi.org/10.1016/j.system.2010.02.001

Fong, C. J., Dillard, J. B., \& Hatcher, M. (2019). Teaching self-efficacy of graduate student instructors: Exploring faculty motivation, perceptions of autonomy support, and undergraduate student engagement. International Journal of Educational Research, 98, 91-105. https://doi.org/10.1016/j.ijer.2019.08.018

Foster, P., \& Ohta, A. S. (2005). Negotiation for meaning and peer assistance in second language classrooms. Applied Linguistics, 26(3), 402-430. https://doi.org/10.1093/applin/ami014

Freeman, D. (2016). Educating Second Language Teachers. Oxford University Press.

Freeman D, Katz A, Garcia Gomez P, \& Burns A (2015). English-for-Teaching: Rethinking teacher proficiency in the classroom. ELT Journal 69(2), 129-39.

Freire, P. (1970). Pedagogy of the oppressed (MB Ramos, Trans.). New York: Continuum, 2007. https://envs.ucsc.edu/internships/internship-readings/freire-pedagogy-of-theoppressed.pdf

Frymier, A., \& Houser, M. (2000). The teacher-student relationship as an interpersonal relationship. Communication Education, 49(3), 207-219. https://doi.org/10.1080/03634520009379209

Gadella Kamstra, L. S. (2020). Analysis of EFL teachers'( de)motivation and awareness in Spain [Doctoral dissertation, University of Essex]. http://repository.essex.ac.uk/id/eprint/26870

Garcés, A., \& Olivera, S. (2014). Communication strategies used by pre-service English teachers of different proficiency levels. HOW, 21(1), 10-25. https://doi.org/10.19183/how.21.1.12

Gass, S. M. (2008). Second language acquisition: An introductory course. Routledge.

Giang, T. (2007). Cross-linguistic analysis of Vietnamese and English with implications for Vietnamese language acquisition and maintenance in the United States. Journal of Southeast Asian American Education and Advancement, 2(1), 1-33. https://doi.org/10.7771/2153-8999.1085

Gibbons, K. (2019). Positive teacher-student relationship building and its effects on motivation, learning, and teacher well-being [Master's thesis, University of Oklahoma]. https://shareok.org/bitstream/handle/11244/319721/2019_Gibbons_Kylie_Thesis.pdf? sequence $=4$

Girón Chávez, A., Cruz Maldonado, E., Cancino, R., \& Villar, M. (2017). The effective use of positive rapport in EFL students. Editorial Universidad Autónoma de Chiapas. 
Goitom, M. (2020). Multilingual research: Reflections on translating qualitative data. The British Journal of Social Work, 50(2), 548-564. https://doi.org/10.1093/bjsw/bcz162

Gorbani, S. (2019). Communicative language teaching approach in lecture presentation. Articol, 8(2), 209-213.

Grant, L. E., \& Nguyen, T. H. (2017). Code-switching in Vietnamese university EFL teachers' classroom instruction: A pedagogical focus. Language Awareness, 26(3), 244-259. https://doi.org/10.1080/09658416.2017.1402915

Guerrero, M. C. M. (2018). Private and inner speech in L2 learning. In J. P. Lantolf, M. E. Poehner, \& M. Swain (Eds.), The Routledge handbook of sociocultural theory and second language development (pp. 152-164). Routledge.

Guest, G., MacQueen, K. M., \& Namey, E. E. (2012). Applied thematic analysis (Vol. 3). Sage. https://doi.org/10.4135/9781483384436

Gupta, D. (2004). CLT in India: Context and methodology come together. ETL Journal, 58(3), 266-269. https://doi.org/10.1093/elt/58.3.266

Gurunathan, N., \& Geethanjali, N. (2016). The merits of communicative language teaching method in relation to L2. Language in India, 16(4), 111-117.

Gustafsson, J. (2017). Single case studies vs. multiple case studies: A comparative study. Halmstad University. https://www.divaportal.org/smash/get/diva2:1064378/FULLTEXT01.pdfig

Ha, A. P. (2019). Communicative Language Teaching (CLT) under the view of Vietnamese Culture. Retrieved March 18 2020, from https://www.academia.edu/38831376/Communicative_Language_Teaching_CLT_un der_the_view_of_Vietnamese_Culture\#: :text=(p.,the\%20requirement $\% 20$ of $\% 20$ glo balization\%20context.

Habash, S. H. (2010). Exploring the relationship between perceived teacher nonverbal immediacy and perceived teacher misbehaviors on student course retention in urban community colleges (Publication Number 3422141) [Doctoral dissertation, University of California, Rvine]. ProQuest Dissertations and Theses.

Hadi, M. J., \& Arante, L. T. (2015). Barriers in teaching English in large classes: Voice of an Indonesian English language teacher (EISSN). ERIC. https://eric.ed.gov/?id=ED582906

Hamzah, S. A. S. (2019). Phonological modification of teacher talk in Indonesian EFL classroom. Retrieved February 25 2020, from https://scholar.google.com/scholar?hl=en\&as_sdt=0\%2C5\&q=PHONOLOGICAL+M ODIFICATION+OF+TEACHER+TALK+IN+INDONESIAN+EFL+CLASSROOM + Syarifa\&btnG $=$

Haneda, M., \& Wells, G. (2008). Learning an additional language through dialogic inquiry. Language and Education, 22(2), 114-136. https://doi.org/10.2167/le730.0

Hanum, N. S. (2017). The importance of classroom interaction in the teaching of reading in junior high school. Universitas Negeri Malang. https://pdfs.semanticscholar.org/de57/829a9e9a31b5aca520f7cb5e4964cc961a9f.pdf 
Harrison, L. J., Clarke, L., \& Ungerer, J. A. (2007). Children's drawings provide a new perspective on teacher-child relationship quality and school adjustment. Early Childhood Research Quarterly, 22(1), 55-71.

Hartono, R. (2017). A critical review of research on negotiation of meaning in second language learning. Jurnal Global Expert, 6(1), 1-7.

Haryono, E. P. (2019). CLT in ESL and EFL context: A challenge to civil engineering teachers in Indonesia. Jurnal Teknik Sipil Terapan (JTST), 1(2), 45-50.

Hassan, Mohammad Kamrul (2014). Teachers' and students' perceived difficulties in implementing communicative language teaching in Bangladesh: A critical study. [Doctoral thesis, the Open University]. https://doi.org/10.21954/ou.ro.0000eefb

Hayati, A., Vahdat, S., \& Khoram, A. (2017). Teacher language awareness revisited: An exploratory study of the level and nature of language awareness of prospective Iranian English teachers. Teaching English Language, 11(1), 69-94. https://doi.org/10.22132/TEL.2017.53531

Hays, D. G., \& Singh, A. A. (2012). Qualitative inquiry in clinical and educational settings. Guilford Press.

Hendricks, C. (2006). Improving schools through action research. Allyn Bacon.

Hennink, M., Hutter, I., \& Bailey, A. (2020). Qualitative research methods. Sage.

Henry, C. T. (2020). The impact of campus environment perceptions and sense of belonging on the persistence decisions of students at a rural community college (Publication Number 27836469) [Doctoral dissertation, Mississippi State University]. ProQuest Dissertations and Theses.

Hernández, M., Robins, R., Widaman, K., \& Conger, R. (2016). School belonging, generational status, and socioeconomic effects on Mexican-origin children's later academic competence and expectations. Journal of Research on Adolescence, 26(2), 241-256. https://doi.org/10.1111/jora.12188

Hidayanti, E. (2019). A descriptive analysis of the roles of teacher's humor toward students' motivation in learning English [Graduating paper, Iain Salatiga]. http://erepository.perpus.iainsalatiga.ac.id/id/eprint/5409

Hoang, Y. P. (2017). Improving English language teaching in Vietnam: Voices from university teachers and students. Current Politics and Economics of South, Southeastern, and Central Asia, 26(3), 285-310.

Huang, Y. G. (2020). Capstone EFL portfolio. [Reseach paper, Vanderbilt University]. https://ir.vanderbilt.edu/bitstream/handle/1803/9919/Capstone\%20final\%20version\% 20-Gia\%20.pdf? sequence $=1 \&$ isAllowed $=y$

Humphrey, C., \& Gendron, Y. (2015). What is going on? The sustainability of accounting academia. Critical Perspectives on Accounting, 26(C), 47-66. https://doi.org/10.1016/j.cpa.2014.09.008

Humphreys, G., \& Wyatt, M. (2014). Helping Vietnamese university learners to become more autonomous. ELT Journal, 68(1), 52-63. https://doi.org/10.1093/elt/cct056 
Hussain, G. (2017). An evaluation of ELT situation in higher education institutions in Punjab, Pakistan. European Journal on English Language Teaching, 2(3), 143-167. https://doi.org/10.5281/zenodo.826888

Huynh, T. P. T. (2017). A review of school bullying in Vietnam-students' voice. In $R e$ thinking teacher professional education: Using research findings for better learning (pp. 288-296). ICET.

https://scholar.google.com/scholar?hl=en\&as_sdt=0\%2C5\&q=\%22A+review+of+sch ool+bullying+in+Vietnam-students\%E2\%80\%99+voice\%22\&btnG $=$

Hyman, M., \& Sierra, J. J. (2016). Open-versus close-ended survey questions. Business Outlook, 14(2), 1-5.

Imran, M., \& Ain, Q. (2019). Effects of non-native instructors' L1, beliefs and priorities on pronunciation pedagogy at secondary level in district Rajanpur, Pakistan. Journal of Language and Cultural Education, 7(2), 108-121. https://doi.org/10.2478/jolace$\underline{2019-0015}$

Irmayani, I., \& Rachmajanti, S. (2017). Lexical features of teacher talk in English classrooms in senior high schools. Dinamika Ilmu, 17(1), 59-70.

Jahanzaib, R., \& Zeeshan, M. (2017). University teachers' and students' beliefs about Grammar Translation method and Communicative Language teaching in Quetta, Balochistan, Pakistan. International Journal of English Linguistics, 7(2), 151-157. https://doi.org/10.5539/ijel.v7n2p151

Jaiprasong, S., \& Pongpairoj, N. (2020). L2 production of English word stress by L1 Thai learners. Language Education and Acquisition Research Network, 13(2), 142-157.

Jamshidnejad, A. (2011). Developing accuracy by using oral communication strategies in EFL interactions. Journal of Language Teaching and Research, 2(3), 530-536. https://doi.org/10.4304/jltr.2.3.530-536

Javadi, F., \& Mohammad, K. (2014). On the relationship between reflective teaching and teachers' burnout. International Journal of Research Studies in Language Learning, 3(4), 85-96. https://doi.org/10.5861/ijrsll.2014.614

Jawhar, S. S. (2018). The use of L1 as a source of humour to facilitate interaction in EFL classrooms. Arab World English Journal, 9(3), 294-310. https://dx.doi.org/10.24093/awej/vol9no3.20

Jensen, E., \& Vinther, T. (2003). Exact repetition as input enhancement in second language acquisition. Language Learning, 53, 373-428. https://doi.org/10.1111/1467$\underline{9922.00230}$

Joe, H.-K., Hiver, P., \& Al-Hoorie, A. H. (2017). Classroom social climate, self-determined motivation, willingness to communicate, and achievement: A study of structural relationships in instructed second language settings. Learning and Individual Differences, 53, 133-144. https://doi.org/10.1016/j.lindif.2016.11.005

Johnson, B. (2014). Educational research: Quantitative, qualitative, and mixed approaches (5th ed.). Sage. 
Johnson, R. B., \& Onwuegbuzie, A. J. (2004). Mixed methods research: A research paradigm whose time has come. Educational Researcher, 33(7), 14-26.

Jones, N. D., \& Brownell, M. T. (2014). Examining the use of classroom observations in the evaluation of special education teachers. Assessment for Effective Intervention, 39(2), 112-124. https://doi.org/10.1177/1534508413514103

Jonsen, K., \& Jehn, K. A. (2009). Using triangulation to validate themes in qualitative studies. Qualitative Research in Organizations and Management: An International Journal, 4(2), 123-150. https://doi.org/ 10.1108/17465640910978391

Kárpáti, L. (2019). Issues of speech fluency and the usage of communication strategies by Hungarian teachers and students. Journal of Languages for Specific Purposes (JLSP), $51-67$.

Kasper, G., \& Færch, C. (1983). Strategies in interlanguage communication. Longman.

Kazemi, A., \& Soleimani, N. (2016). On the relationship between EFL teachers' classroom management approaches and the dominant teaching style: A mixed method study. Iranian Journal of Language Teaching Research, 4(2), 87-103.

Kazi, A. M., \& Khalid, W. (2012). Questionnaire designing and validation. The Journal of the Pakistan Medical Association, 62(5), 514-516.

Kendall, L. (2014). The conduct of qualitative interviews: Research questions, methodological issues, and researching online. In J. Coiro, M. Knobel, C. Lankshear, \& D. J. Leu (Eds.), Handbook of research on new literacies (pp. 151-168). Routledge.

Kerr, P. (2019). The use of L1 in English language teaching. Cambridge University Press.

Khader, F. R. (2012). Teachers' pedagogical beliefs and actual classroom practices in social studies instruction. American International Journal of Contemporary Research, 2(1), 73-92.

Khaliliaqdam, S. (2014). ZPD, scaffolding and basic speech development in EFL context. Procedia - Social and Behavioral Sciences, 98(2014), 891-897. https://doi.org/10.1016/j.sbspro.2014.03.497

Khodamoradi, A., Maghsoudi, M., \& Talebi, S. H. (2019). Student teachers' and educators' perceptions of educator interpersonal behavior at teacher education university in Iran. Journal of Modern Research in English Language Studies, 6(4), 137-107.

Kieu, K. (2010). Use of Vietnamese in English language teaching in Vietnam: Attitudes of Vietnamese university teachers. English Language Teaching, 3(2), 119-128. https://doi.org/10.5539/elt.v3n2p119

Kim, J., \& Tatar, B. (2017). Nonnative English-speaking professors' experiences of Englishmedium instruction and their perceived roles of the local language. Journal of Language, Identity \& Education, 16(3), 157-171. https://doi.org/10.1080/15348458.2017.1295811 
Kim, J. S. (2014). Effects of English writing as a pre-speaking activity on Korean EFL university learner's speaking ability and anxiety. English Language \& Literature Teaching, 20(1), 329-356.

Knight, P. (2001). The development of EFL methodology. In C. Candlin \& N. Mercer (Eds.), English language teaching in its social context (pp. 147-166). Routledge.

Korpershoek, H., Canrinus, E. T., Fokkens-Bruinsma, M., \& De Boer, H. (2019). The relationships between school belonging and students' motivational, social-emotional, behavioural, and academic outcomes in secondary education: A meta-analytic review. Research Papers in Education, 1-40. https://doi.org/10.1080/02671522.2019.1615116

Korstjens, I., \& Moser, A. (2017). Series: Practical guidance to qualitative research. Part 4: Trustworthiness and publishing. The European Journal of General Practice, 24(1), 120-124. https://doi.org/10.1080/13814788.2017.1375092

Kovac, M. M. (2016). Repetitions as a communication strategy: A case study. Studies in English Language Teaching, 4(1), 87-103. https://doi.org/10.22158/selt.v4n1p87

Kozulin, A. (2003). Psychological tools and mediated learning. In B. G. A.Kozulin, V.Ageyev \& S. Miller (Ed.), Vygotsky's educational theory in cultural context (pp. 15-38). Cambridge University Press.

Krane, V., Bengt, K., Ness, O., \& Kim, H. (2016). Teacher-student relationship, student mental health, and dropout from upper secondary school: A literature review. Scandinavian Psychologist, 3, e11. https://doi.org/10.15714/scandpsychol.3.e11

Krashen, S. (1980). The input hypothesis. In Alatis (Ed.), Current issues in bilingual education (pp. 144-158). Georgetown University Press.

Krashen, S. D. (1988). Second language acquisition and second language learning. Prentice Hall.

Krishnan, I. A., Ramalingam, S. J., Ching, H. S., \& Maruthai, E. (2018). The functions of communication strategies: An analysis of Chinese EFL learners' transactional practice. Journal of Language and Communication, 5(2), 151-159.

Kumaravadivelu, B. (2003). Beyond methods: Macrostrategies for language teaching. Yale University Press.

Kurt, P. Y., \& Kurt, H. (2018). Instructional practices that motivate students to learn English. Journal of Education and Future, 14, 119-130.

Kwon, Y. (2017). A study of Thai teachers' perceptions toward the implementation of Communicative Language Teaching of English. HRD Journal, 8(1), 114-125.

Lam, P. W. (2010). Discourse particles in corpus data and textbooks: The case of Well. Applied Linguistics, 31(2), 260-281. https://doi.org/10.1093/applin/amp026

Lancaster, C. (2020). A study of sense of belonging and its relationship with engagement, persistence, and intersectionality in higher education (Publication Number 27955011) [Doctoral dissertation, Eastern Michigan University]. ProQuest Dissertations. 
Lantolf, J., Thorne, S., \& Poehner, M. (2014). Sociocultural theory and second language development. In B. Van Patten \& J. Williams (Eds.), Theories in second language acquisition (pp. 207-226). Routledge.

Lantolf, J., \& Thorne, S. L. (2007). Sociocultural theory and second language learning. In B. Van Patten \& J. Williams (Eds.), Theories in second language acquisition (pp. 201224). Lawrence Erlbaum.

Lantolf, J. P. (2000). Second language learning as a mediated process. Language teaching, 33(2), 79-96. https://doi.org/10.1017/S0261444800015329

Le, T. T., \& Chen, S. (2019). Globalisation and Vietnamese foreign language education. In J. Albright (Ed.), English tertiary education in Vietnam (1st ed., pp. 16-27). Routledge.

Le, V. C. (2011). Form-focused instruction: A case study of Vietnamese teachers beliefs and practices (Publication Number 9783659120220) [Doctoral thesis, University of Waikato]. https://hdl.handle.net/10289/5253

Le, V. C. (2020). Remapping the teacher knowledge-base of language teacher education: A Vietnamese perspective. Language Teaching Research, 24(1), 71-81. https://doi.org/10.1177/1362168818777525

Lee, J., \& Choi, E. (2019). Mismatches between student and teacher perceptions of current and ideal foreign language classroom practices. Korean Journal of Applied Linguistics, 35(3), 77-102. https://doi.org/10.17154/kjal.2019.9.35.3.77

Lee, J. H., \& Lo, Y. Y. (2017). An exploratory study on the relationships between attitudes toward classroom language choice, motivation, and proficiency of EFL learners. System, 67, 121-131. https://doi.org/10.1016/j.system.2017.04.017

Len, K. (2018). Classroom communication techniques: A tool for pupils' participation in the learning process across the curriculum. Creative Education, 9, 535-548. https://doi.org/10.4236/ce.2018.93037

Lengluan, N. (2008). An analysis of conversational exchanges between grade 12 Thai sudents and native English speakers via a chat program: Strategies of negotiation for meanings [Unpublished master's thesis]. Srinakarinwirot University, Thailand. http://thesis.swu.ac.th/swuthesis/Tea_Eng_For_Lan(M.A.)/Nattanan_L.pdf

Leoanak, S. P. P., \& Amalo, B. K. (2018). Teachers' beliefs and perceptions of code switching in English as foreign language classroom. SHS Web of Conferences, Indonesia.

Levin, T., \& Wadmany, R. (2008). Teachers' views on factors affecting effective integration of information technology in the classroom: Developmental scenery. Journal of Technology and Teacher Education, 16(2), 233-263.

Levine, G. S. (2014). Principles for code choice in the foreign language classroom: A focus on grammaring. Language Teaching, 47(3), 332-348.

https://doi.org/10.1017/S0261444811000498

Li, D. (2001). Teachers' perceived difficulties in introducing the communicative approach in South Korea. In D. R. Hall \& A. Hewings (Eds.), Innovation in English language teaching: A reader (pp. 149-168). Routledge. 
Li, D., \& Zhang, L. (2020). Exploring teacher scaffolding in a CLIL-framed EFL intensive reading class: A classroom discourse analysis approach. Language Teaching Research, 00(0) 1-28. https://doi.org/10.1177/1362168820903340

Li, J., Bjornson, F. O., Conradi, R., \& Kampenes, V. B. (2006). An empirical study of variations in COTS-based software development processes in the Norwegian IT industry. Empirical Software Engineering, 11(3), 433-461. https://doi.org/10.1007/s10664-006-9005-5

Li, L. (2020). Language teacher cognition: A sociocultural perspective (1st ed.). Palgrave Macmillan UK. https://doi.org/10.1057/978-1-137-51134-8

Li, L., \& Walsh, S. (2011). 'Seeing is believing': Looking at EFL teachers' beliefs through classroom interaction. Classroom Discourse, 2(1), 39-57. https://doi.org/10.1080/19463014.2011.562657

Li, M.-Y., \& Wu, T.-C. (2017). Creating an EMI program in international finance and business management. In W. Tsou \& S.-M. Kao (Eds.), English as a medium of instruction in higher education: Implementations and classroom practices in Taiwan (pp. 21-38). Springer. https://doi.org/10.1007/978-981-10-4645-2_2

Li, Y. (2011). Translating interviews, translating lives: Ethical considerations in crosslanguage narrative inquiry. TESL Canada Journal, 28(5), 16-30. https://doi.org/10.18806/tesl.v28i0.1079

Liamputtong, P. (2011). Focus group methodology: Principles and practices. Sage.

Liang, A. (2013). The study of second language acquisition under socio-cultural theory. American Journal of Educational Research, 1, 162-167. https://doi.org/10.12691/education-1-5-3

Lightbown, P., \& Spada, N. M. (1999). How languages are learned (2nd ed.). Oxford University Press.

Liu, D., Ahn, G.-S., Baek, K.-S., \& Han, N.-O. (2004). South Korean high school English teachers' code switching: Questions and challenges in the drive for maximal use of English in teaching. TESOL Quarterly, 38(4), 605-638.

https://doi.org/10.2307/3588282

Lo, Y. Y. (2015). How much L1 is too much? Teachers' language use in response to students' abilities and classroom interaction in Content and Language Integrated Learning. International Journal of Bilingual Education and Bilingualism, 18(3), 270-288. https://doi.org/10.1080/13670050.2014.988112

Lobo, A., \& Gurney, L. (2014). What did they expect? Exploring a link between students' expectations, attendance and attrition on English language enhancement courses. Journal of Further and Higher Education, 38(5), 730-754. https://doi.org/10.1080/0309877X.2013.817002

Long, M. H. (1983). Native speaker/non-native speaker conversation and the negotiation of comprehensible input. Applied Linguistics, 4(2), 126-141. https://doi.org/10.1093/applin/4.2.126 
Long, M. H. (1996). The role of the linguistic environment in second language acquisition. In W. Ritchie and T. Bhatia (Ed.), Handbook of research on second language acquisition (pp.413-468). Academic.

Luo, J., \& Garner, M. (2017). The challenges and opportunities for English teachers in teaching ESP in China. Journal of Language Teaching and Research, 8(1), 81-86. https://doi.org/10.17507/jltr.0801.10

Luo, M., Main, S., Lock, G., Joshi, R. M., \& Zhong, C. (2020). Exploring Chinese EFL teachers' knowledge and beliefs relating to the teaching of English reading in public primary schools in China. Dyslexia, 26(3), 266-285. https://doi.org/10.1002/dys.1630

Lyster, R. (2018). Roles for corrective feedback in second language instruction. In C. A. Chapelle (Ed.), The encyclopedia of applied linguistics (pp. 1-6). John Wiley \& Sons. https://doi.org/10.1002/9781405198431.wbeal1028.pub2

Ma, L., Du, X., Hau, K.-T., \& Liu, J. (2018). The association between teacher-student relationship and academic achievement in Chinese EFL context: A serial multiple mediation model. Educational Psychology, 38(5), 687-707. https://doi.org/10.1080/01443410.2017.1412400

Ma, L. P. F. (2019). Examining the functions of L1 use through teacher and student interactions in an adult migrant English classroom. International Journal of Bilingual Education and Bilingualism, 22(4), 386-401.

https://doi.org/10.1080/13670050.2016.1257562

Macaro, E. (2009). Teacher use of codeswitching in the second language classroom: Exploring 'optimal'use. In M. Turnbull \& J. Dailey-O'Cain (Eds.), First language use in second and foreign language learning (pp. 35-49). Multilingual Matters. https://doi.org/10.21832/9781847691972

Macaro, E., Curle, S., Pun, J., An, J., \& Dearden, J. (2018). A systematic review of English medium instruction in higher education. Language Teaching, 51(1), 36-76.

Mackey, A. (2005). The handbook of second language acquisition. Studies in Second Language Acquisition, 27(4), 619-623. https://doi.org/10.1017/S0272263105210288

Mahbubeh, Y. (2019). On the comparison of L2 poetry teaching approaches: L1 use in the Iranian EFL classrooms. International journal of English language and translation studies, 7(3), 67-79.

Mahmadun Nuby, H., Ab Rashid, R., Rahman, M., \& Hasan, R. (2020). Communicative language teaching in Bangladeshi rural schools. Universal Journal of Educational Research, 8(2), 622-630. https://doi.org/10.13189/ujer.2020.080235

Mai, H. T. N. (2017). Contextual factors affecting the implementation of communicative language teaching in Vietnam. EFL Journal, 2(2), 103-113.

Mai, N. K., \& Iwashita, N. (2012). A comparison of learners' and teachers' attitudes toward communicative language teaching at two universities in Vietnam. University of Sydney Papers in TESOL, 7, 25-49.

Marshall, C., \& Rossman, G. B. (2014). Designing qualitative research. Sage. 
Marzuki, M., Prayogo, J., \& Wahyudi, A. (2016). Improving the EFL learners' speaking ability through interactive storytelling. Dinamika Ilmu, 16(1), 15-31.

https://doi.org/10.21093/di.v16i1.307

McNeill, D. (1994). Hand and mind: What gestures reveal about thought. Bibliovault OAI Repository, the University of Chicago Press, 27, 105-133. https://doi.org/10.2307/1576015

Meier, C. (2010). The challenge of equipping student teachers to manage their perceptions of learner diversity in South African schools. Journal for New Generation Sciences, $8(1), 157-176$.

Mercer, S., Oberdorfer, P., \& Saleem, M. (2016). Helping language teachers to thrive: Using positive psychology to promote teachers' professional well-being. In D. GabryśBarker \& D. Gałajda (Eds.), Positive psychology perspectives on foreign language learning and teaching (pp. 213-229). Springer. https://doi.org/10.1007/978-3-319$\underline{32954-3 \_12}$

Merriam, S. B. (2009). Qualitative research a guide to design and implementation. JosseyBass.

Messner, M., Moll, J., \& Strömsten, T. (2017). Credibility and authenticity in qualitative accounting research. In Z. Hoque, L. D. Parker, M. A. Covaleski, \& K. Haynes (Eds.), The Routledge companion to qualitative accounting research methods (pp. 432-443). Routledge.

Mariani, L. (2010). Communication strategies: Learning and teaching how to manage oral interaction. Lulu.com.

Miles, M. B., \& Huberman, A. M. (1994). Qualitative data analysis: An expanded sourcebook. Sage.

Mills, A. J., Durepos, G., \& Wiebe, E. (2010). Encyclopedia of case study research (Vol. 1). Sage. https://doi.org/10.4135/9781412957397

Mockler, N. (2014). When 'research ethics' become 'everyday ethics': The intersection of inquiry and practice in practitioner research. Educational Action Research, 22(2), 146-158. https://doi.org/10.1080/09650792.2013.856771

Modupeola, O. R. (2013). Code-switching as a teaching strategy: Implication for English language teaching and learning in a multilingual society. IOSR Journal of Humanities and Social Science, 14(3), 92-94. https://doi.org/10.9790/1959-1439294

Mohammed Elhassan, I. B., \& Adam, M. I. (2017). The impact of dialogic teaching on English language learners' speaking and thinking skills. Arab World English Journal 8(4), 49-67. https://doi.org/10.24093/awej/vol8no4.4

Mohebbi, H. A., \& Alavi, S. M. H. (2014). An investigation into teachers' first language use in a second language learning classroom context: A questionnaire-based study.

Bellaterra Journal of Teaching \& Learning Language \& Literature, 7(4), 57-73. https://doi.org/10.5565/rev/jt13.539 
Moodie, I. (2016). The anti-apprenticeship of observation: How negative prior language learning experience influences English language teachers' beliefs and practices. System, 60, 29-41. https://doi.org/10.1016/j.system.2016.05.011

Morgan, A. (2017). Cultivating critical reflection: Educators making sense and meaning of professional identity and relational dynamics in complex practice. Teaching Education, 28(1), 41-55. https://doi.org/10.1080/10476210.2016.1219335

Morgan, D. L. (2007). Paradigms lost and pragmatism regained: Methodological implications of combining qualitative and quantitative methods. Journal of Mixed Methods Research, 1(1), 48-76. https://doi.org/10.1177/2345678906292462

Morse, J. (2015). Critical analysis of strategies for determining rigor in qualitative inquiry. Qualitative health research, 25(9), 1212-1222. https://doi.org/10.1177/1049732315588501

Motallebzadeh, K., Hosseinnia, M., \& Domskey, J. G. H. (2017). Peer observation: A key factor to improve Iranian EFL teachers' professional development. Cogent Education, 4(1), 1277456. https://doi.org/10.1080/2331186X.2016.1277456

Muhlisin, M., \& Widyanto, A. (2019). An analysis on teacher's communication strategies in teaching speaking at MTs NW Bonjeruk. JOLLT Journal of Languages and Language Teaching, 7(2), 154-165.

Mulisa, F., \& Kassahun, S. (2019). Teachers' expectation of students' achievement as a predictor of motivation to teach in Ethiopian secondary schools: Implication for quality education. Ethiopian Journal of Education and Sciences, 14(2), 28-47.

Munn, Z., Porritt, K., Lockwood, C., Aromataris, E., \& Pearson, A. (2014). Establishing confidence in the output of qualitative research synthesis: the ConQual approach. BMC Medical Research Methodology, 14(1), 108-114. https://doi.org/10.1186/1471$\underline{2288-14-108}$

Muñoz-Basols, J. (2005). Learning through humor: Using humorous resources in the teaching of foreign languages. The A.T.I.S Bulletin, 42-46.

Murphey, T. (1990). The song stuck in my head phenomenon: A melodic Din in the LAD?". System, 18(1), 53-64.

Murtaza, A. (2016). Students' perceptions of English language instruction (EMI) at a private university in Bangladesh: A survey. Language in India, 16(11), 1-11.

Museus, S. D., Yi, V., \& Saelua, N. (2017). The impact of culturally engaging campus environments on sense of belonging. The Review of Higher Education, 40(2), 187215. https://doi.org/10.1353/rhe.2017.0001

Mutlu, G., \& Yıldırım, A. (2019). Learning environment perceptions and student background variables as determinants of persistence in EFL learning. Sage Open, 9(4), 1-17. https://doi.org/10.1177/2158244019898805

Mwendwa, E. (2020). Relationship between school feeding programme and pupils' transition from a class to another in public primary schools in Kitui county, Kenya. International Journal of Innovative Research and Advanced Studies (IJIRAS), 7(8), 19-28. 
Naidoo, D. (2010). Losing the "purity" of subjects? Understanding teachers' perceptions of integrating subjects into learning areas. Education as Change, 14(2), 137-153. https://doi.org/10.1080/16823206.2010.518001

Nakatani, Y. (2006). Developing an oral communication strategy inventory. The Modern Language Journal, 90(2), 151-168. https://doi.org/10.1111/j.1540-4781.2006.00390.x

Nakatsukasa, K., \& Loewen, S. (2014). A teacher's first language use in form-focused episodes in Spanish as a foreign language classroom. Language Teaching Research, 19(2), 133-149. https://doi.org/10.1177/1362168814541737

Namaziandost, E., \& Nasri, M. (2019). A meticulous look at Long's (1981) interaction hypothesis: Does it have any effect on speaking skill?. Journal of Applied Linguistics and Language Research, 6(2), 218-230.

Namaziandost, E., Nasri, M., \& Ziafar, M. (2019). Comparing the impacts of various inputs (I+ $1 \& \mathrm{I}-1)$ on pre-intermediate EFL learners' reading comprehension and reading motivation: The case of Ahvazi learners. Asian-Pacific Journal of Second and Foreign Language Education, 4(1), 4-13. https://doi.org/10.1186/s40862-019-0079-1

Nassar, M., Al-Khadash, H. A., \& Sangster, A. (2011). The diffusion of activity-based costing in Jordanian industrial companies. Qualitative Research in Accounting \& Management, 8, 180-200. https://doi.org/10.1108/11766091111137573

Ngo, B. (2020). Vietnamese: An essential grammar. Taylor \& Francis Group.

Ngo, H., Spooner-Lane, R., \& Mergler, A. (2017). A comparison of motivation to learn English between English major and non-English major students in a Vietnamese university. Innovation in Language Learning and Teaching, 11(2), 188-202. https://doi.org/10.1080/17501229.2015.1094076

Nguyen, H. T., Hamid, M. O., \& Moni, K. (2016). English-medium instruction and selfgovernance in higher education: The journey of a Vietnamese university through the institutional autonomy regime. Higher Education, 72(5), 669-683. https://doi.org/10.1007/s10734-015-9970-y

Nguyen, H. T. M., \& Bui, T. (2016). Teachers' agency and the enactment of educational reform in Vietnam. Current Issues in Language Planning, 17(1), 88-105. https://doi.org/10.1080/14664208.2016.1125664

Nguyen, N. T. (2019). Cultural modalities of Vietnamese higher education. In N. T. Nguyen \& L. T. Tran (Eds.), Reforming Vietnamese higher education: Global forces and local demands (pp. 17-33). Springer. https://doi.org/10.1007/978-981-13-8918-4_2

Nguyen, N. T., Grainger, P., \& Carey, M. (2016). Code-switching in English language education: Voices from Vietnam. Theory and Practice in Language Studies, 6(7), $1333-1340$.

Nguyen, T. B. (2015). Nonverbal communication in Vietnamese EFL classrooms. Jounal of Science, Ho Chi Minh University of Education, 4(69), 185-191.

Nguyen, T. B. H., Sun, H. J., \& Yang, Y. (2010). English-only classrooms: Ideology versus reality [Paper presentation]. AARE Annual Conference 2010, Melburn. https://www.aare.edu.au/data/publications/2010/1755HoangJangYang.pdf 
Nguyen, T. H. (2013). Vietnamese University EFL teachers' code-switching in classroom instruction [Doctoral dissertation, Auckland University of Technology]. Open Theses \& Dissertations. http://hdl.handle.net/10292/7619

Nguyen, T. H., Fehring, H., \& Warren, W. (2014). EFL teaching and learning at a Vietnamese university: What do teachers say? English Language Teaching, 8, 31-43. https://doi.org/10.5539/elt.v8n1p31

Nguyen, T. T., \& Nguyen, T. K. T. (2016). Oral English communication strategies among Vietnamese non-majors of English at intermediate level. American Journal of Educational Research, 4(6), 283-287. https://doi.org/10.12691/education-4-3-9

Nhapulo, M. (2013). Teacher and learner beliefs and expectations about English language teaching and learning at a Mozambican university. Afrika Focus, 26(2), 81-109. https://doi.org/10.21825/af.v26i2.4913

Ni, H. (2012). The effects of affective factors in SLA and pedagogical implications. Theory and Practice in Language Studies, 2(7), 1508-1513. https://doi.org/10.4304/tpls.2.7.1508-1513

Nijat, N., Atifnigar, H., Chandran, K., Selvan, S. L. T., \& Subramonie, V. (2019). Psychological factors that affect English speaking performance among Malaysian primary school pupils. American International Journal of Education and Linguistics Research, 2(2), 55-68. https://doi.org/10.46545/aijelr.v2i2.117

Nilubol, K. (2020). The impacts of L1 integration towards general English classroom of Thai public university undergraduate students: An account of learning motivation perspective. Language Education and Acquisition Research Network Journal, 13(2), 426-444.

NLĐ. (2020). Những trường ĐH có chuẩn đầu ra ngoại ngữ cao nhất TP HCM. Retrieved October 19, 2018 from https://phunuvatiepthi.net/nhung-truong-dh-co-chuan-dau-rangoai-ngu-cao-nhat-tp-hcm/

Nowell, L., Norris, J., White, D., \& Moules, N. (2017). Thematic Analysis: Striving to meet the trustworthiness criteria. International Journal of Qualitative, 16(1), 1-13. https://doi.org/10.1177/1609406917733847

Nukuto, H. (2017). Code choice between L1 and the target language in English learning and teaching: A case study of Japanese EFL classrooms. Acta Linguistica Hafniensia, 49(1), 1-19. https://doi.org/10.1080/03740463.2017.1316631

Nurpahmi, S. (2017). Teacher talk in classroom interaction. ETERNAL (English, Teaching, Learning and Research Journal), 3(1), 34-43. https://doi.org/10.24252/Eternal.V31.2017.A4

O'Bannon, B. W., \& Thomas, K. (2014). Teacher perceptions of using mobile phones in the classroom: Age matters. Computers \& Education, 74, 15-25.

O'Toole, J. (2010). Educational research: Creative thinking \& doing. Oxford University Press. 
Obeidat, M. (2020). EFL undergraduate students' perspective about the characteristics of the effective instructor at Hashemite university in Jordan. International Journal of Higher Education, 9(3), 28-38. https://doi.org/10.5430/ijhe.v9n5p193

Odlin, T. (1989). Language transfer: Cross-linguistic influence in language learning. Cambridge University Press. https://www.cambridge.org/core/books/language$\underline{\text { transfer/0A19422B8005A10AFC282615FD542519 }}$

Ohta, A. (2000). Rethinking interaction in SLA: Developmentally appropriate assistance in the zone of proximal development and the acquisition of L2 grammar. In J. P. Lantolf (Ed.), Sociocultural theory and second language learning (pp. 51-78). Oxford University Press.

Ohta, A. S. (2017). Sociocultural theory and second/foreign language education. In N. Van Deusen-Scholl \& S. May (Eds.), Second and foreign language education (pp. 57-68). Springer International Publishing. https://doi.org/10.1007/978-3-319-02246-8_6

Okello, L. M., \& Olung'a, T. M. (2020). A survey of kindergarten through 12th grade teacher perceptions of the learning environment in the mid-atlantic region of the United States. International Journal of Novel Research in Education and Learning, 7(2), 68 82.

Ornstein, D. S., \& Weiss, B. (1991). Statistical properties of chaotic systems. Bulletin of the American Mathematical Society, 24(1), 11-116.

Owen, E., Razali, A. B., \& Elhaj, I. (2019). From the past to the present: A view of teaching English as a foreign language (EFL) in Libya and the role of communicative language teaching (CLT) approach. International Journal of Academic Research in Business and Social Sciences, 9(2), 459-476. https://doi.org/10.6007/IJARBSS/v9-i2/5580

Park, M., \& Sung, Y. K. (2013). Teachers' perceptions of the recent curriculum reforms and their implementation: What can we learn from the case of Korean elementary teachers?. Asia Pacific Journal of Education, 33(1), 15-33.

Panhwar, D. A. H., Baloch, S., \& Khan, S. (2017). Making communicative language teaching work in Pakistan. International Journal of English Linguistics, 7(3), 226-234. https://doi.org/10.5539/ijel.v7n3p226

Parab, V. V. (2020). Global technique of teaching English through English: Pedagogy, proficiency and performance. A Global Journal of Interdisciplinary Studies, 3(2), 4966.

Patton, M. Q. (2014). Qualitative research \& evaluation methods: Integrating theory and practice (4th ed.). Sage.

Peacock, S., Cowan, J., Irvine, L., \& Williams, J. (2020). An exploration into the importance of a sense of belonging for online learners. International Review of Research in Open and Distributed Learning, 21(2), 18-35. https://doi.org/10.19173/irrodl.v20i5.4539

Pham, H. (2015). Common errors in writing journals of the English-major students at Ho Chi Minh city open university. Journal of Science Ho Chi Minh City Open University, 2(14), 60-69. http://journalofscience.ou.edu.vn/index.php/socien/article/viewFile/315/244 
Pham, H. H. (2004). Trained in the West, teaching in the East: Vietnamese teachers returning from TESOL courses abroad [Doctoral Dissertation, University of Melbourne, Victoria, Australia]. http://hdl.handle.net/11343/39930

Pham, H. H. (2007). Communicative language teaching: Unity within diversity. ELT Journal, 61(3), 193-201. https://doi.org/10.1093/elt/ccm026

Pham, H. P. Q. (2017). Group dynamics: Building a sense of belonging in the EFL classroom. English Teaching Forum, 14-21.

Pham, T. H. N. (2014). The impact of third party presence on the motivational concerns underlying linguistic politeness behavior in English-speaking intercultural contexts. Journal for the Study of English Linguistics, 2(1), 19-33. https://doi.org/10.5296/jsel.v2i1.5833

Pham, T. H. N. (2018). General English proficiency or English for teaching? The preferences of in-service teachers. RELC Journal, 49(3), 339-352. https://doi.org/10.1177/0033688217691446

Pham, T. N. A. (2017). Emotion in English as an additional language oral communication: Vietnamese English language teachers and students [Doctoral dissertation, The University of Waikato]. https://researchcommons.waikato.ac.nz/bitstream/handle/10289/11440/thesis.pdf?seq uence $=4$

Pham, T. T. L. (2011). Teacher's humor use in the classroom and students' perceptions of its effectiveness and appropriateness [Undergraduate's paper, Vietnam national university, Hanoi university of languages and international]. http://repository.ulis.vnu.edu.vn/bitstream/ULIS_123456789/1864/1/Ph\%E1\%BA\%A 1m\%20Th\%E1\%BB\%8B\%20Th\%C3\%B9y\%20Linh.\%20QH\%202007.\%20F1.\%20 E1.\%20GHP.\%20Teacher\%27s\%20humor\%20us\%20\%28pdf.io\%29.pdf

Phipps, S., \& Borg, S. (2009). Exploring tensions between teachers' grammar teaching beliefs and practices. System, 37(3), 380-390. https://doi.org/10.1016/j.system.2009.03.002

Pianta, R. C., Steinberg, M. S., \& Rollins, K. B. (1995). The first two years of school: Teacher-child relationships and deflections in children's classroom adjustment. Development and Psychopathology, 7(2), 295312.

Poehner, M., \& Infante, P. (2015). Mediated Development: Inter-psychological activity for L2 education. Language and Sociocultural Theory, 2(2), 161-183. https://doi.org/10.1558/lst.v2i2.26982

Polit, D. F., Beck, C. T., \& Polit, D. (2012). Generating and assessing evidence for nursing practice (9th ed.). Wolters Kluwer Health.

Poulisse, N. 1990. The use of compensatory strategies by Dutch learners of English. Berlin.

Poulisse, N. (1993). A theoretical account of lexical communication strategies. John Benjamins.

Pourtoussi, Z., Ghanizadeh, A., \& Mousavi, V. (2018). A qualitative in-depth analysis of the determinants and outcomes of EFL teachers' motivation and demotivation. International Journal of Instruction, 11(4), 175-190. 
Putri, D. S. (2015). The analysis of teacher talk and the characteristic of classroom interaction in English as a foreign language classroom. Journal of English and Education, 3(2), $16-27$.

Queirós, A., Faria, D., \& Almeida, F. (2017). Strengths and limitations of qualitative and quantitative research methods. European Journal of Education Studies, 3(9), 369387. https://doi.org/10.5281/zenodo.88708

Rahmadani, D. (2016). Students' perception of English as a medium of instruction (EMI) in English classroom. Journal on English as a Foreign Language, 6(2), 131-144. https://doi.org/10.23971/jefl.v6i2.432

Rahman, A. (2017). Emerging factors of communicative language teaching (CLT) and its application in Indonesian English as a foreign language (EFL) classrooms. LANGKAWI, 3(2), 169-174. https://doi.org/10.31332/lkw.v3i2.587

Rahmani Doqaruni, V. (2017). Communication strategies in experienced vs. inexperienced teachers' talk: A sign of transformation in teacher cognition. Innovation in Language Learning and Teaching, 11(1), 17-31. https://doi.org/10.1080/17501229.2015.1009071

Rezaei, S., Mozaffari, F., \& Hatef, A. (2011). Corrective feedback in SLA: Classroom practice and future directions. International Journal of English Linguistics, 1(1), 2129.

Riazi, A. M. (2016). The Routledge encyclopedia of research methods in applied linguistics: Quantitative, qualitative, and mixed-methods research. Routledge.

Richards, J. C. (1998). Beyond training: Perspectives on language teacher education. Cambridge University Press.

Richards, J. C. (2006). Communicative language teaching today. Cambridge University Press.

Richards, J C. \& Schmidt, R. (2010). Longman Dictonary of language teaching and applied linguistics (4th ed). Pearson.

Riedel, R., Vialle, W., Pearson, P., \& Oades, L. G. (2020). Quality learning and positive education practice: The student experience of learning in a school-wide approach to positive education. International Journal of Applied Positive Psychology, 5(1), 53-75. https://doi.org/10.1007/s41042-020-00029-5

Riordan, E. (2018a). Language for teaching purposes bilingual classroom discourse and the non-native speaker language teacher (1st ed.). Springer International Publishing. https://doi.org/10.1007/978-3-319-71005-1

Riordan, E. (2018b). The non-native speaker language teacher. In E. Riordan (Ed.), Language for teaching purposes: Bilingual classroom discourse and the non-native speaker language teacher (pp. 113-145). Springer International Publishing. https://doi.org/10.1007/978-3-319-71005-1_5

Ritchie, G. D. (2010). Linguistic factors in humour. In D. Chiaro (Ed.), Translation, humour and literature (Vol. 1, pp. 33-48). (Continuum Advances in Translation). Continuum. 
Roman Etxebarrieta, G., Pérez-Izaguirre, E., \& Langarika-Rocafort, A. (2020). Teaching minority languages in multiethnic and multilingual environments: Teachers' perceptions of students' attitudes toward the teaching of basque in compulsory education. Education Sciences, 10(2), 25-45. https://doi.org/10.3390/educsci10020025

Roni, S. M., Merga, M. K., \& Morris, J. E. (2020). Conducting quantitative research in education. Springer.

Roohani, A., Forootanfar, F., \& Hashemian, M. (2017). Effect of input vs. collaborative output tasks on Iranian intermediate EFL learners' grammatical accuracy and willingness to communicate. Research in Applied Linguistics, 8(2), 71-92.

Roorda, D. L., Koomen, H. M., Spilt, J. L., \& Oort, F. J. (2011). The influence of affective teacher-student relationships on students' school engagement and achievement: A meta-analytic approach. Review of Educational Research, 81(4), 493-529.

Rose, H., \& Galloway, N. (2019). Global Englishes for Language Teaching. Cambridge University Press. https://doi.org/10.1017/9781316678343

Sahlim, M. (2018). The functional characteristics of teacher talks in English classroom. Media Pendidikan dan Sosial Kemasyarakatan, 13(2), 134-147.

Salama, A. (2018). Exploring Egyptian EFL Teachers' Perceptions of Teacher Leadership [Master's thesis, the American University in Cairo]. AUC Knowledge Fountain. https://fount.aucegypt.edu/etds/1350

Salifu, I., \& Agbenyega, J. S. (2013). Viewing teacher motivation in the Ghana education service through a postcolonial lens. Current Issues in Education, 16(3), 1-14.

Sammaknejad, A., \& Marzban, A. (2016). An analysis of teachers' self-reflection on classroom management. Theory and Practice in Language Studies, 6(1), 84-89. http://dx.doi.org/10.17507/tpls.0601.11

Sánchez, C. A. G., De González, B. S. G., \& Martínez, C. d. J. L. (2013). The impact of teacher-student relationships on EFL learning. HOW, 20(2013), 116-129.

Saville-Troike, M. (1988). Private speech: Evidence for second language learning strategies during the 'silent' period. Journal of Child Language, 15(3), 567-590. https://doi.org/10.1017/S0305000900012575

Saville-Troike, M. (2012). Introducing second language acquisition (2nd ed.). Cambridge University Press. https://doi.org/10.1017/CBO9780511888830

Savran Celik, S., \& Aydin, S. (2018). A review of research on the use of native language in EFL classes. Literacy Trek, 4(2), 1-14.

Scaffidi, M. A., Walsh, C. M., Khan, R., Parker, C. H., Al-Mazroui, A., Abunassar, M., Grindal, A. W., Lin, P., Wang, C., Bechara, R., \& Grover, S. C. (2019). Influence of video-based feedback on self-assessment accuracy of endoscopic skills: A randomized controlled trial. Endoscopy International Open, 7(5), E678-E684. https://doi.org/10.1055/a-0867-9626 
Schmidt, R. (2001). Attention. In P. Robinson (Ed.), Cognition and Second Language Instruction (pp. 3-32). Cambridge University Press. https://doi.org/10.1017/CBO9781139524780.003

Schumm, J. S., \& Arguelles, M. E. (2006). Reading assessment and instruction for all learners. Guilford Press.

Seidman, I. (2006). Interviewing as qualitative research: A guide for researchers in education and the social sciences (3rd ed.). Teachers College Press.

Selvaraj, M., Aziz, A. A., Mariam, S. T., \& Pahat, B. (2020). Utilizing flow chart in writing narrative essay: English as second language students' perceptions. International Journal of Academic Research in Business and Social Sciences, 10(3), 1-16. https://doi.org/10.6007/IJARBSS/v10-i3/7017

Selvi, A. F. (2014). Myths and misconceptions about nonnative English speakers in the TESOL (NNEST) movement. TESOL Journal, 5(3), 573-611.

Sevim, I., \& Turhanli, I. (2019). Teachers' opinions on the use of L1 in EFL classes. Journal of Language and Linguistic Studies, 15(3), 861-875.

Shabir, M. (2017). Student-teachers' beliefs on the use of L1 in EFL classroom: A global perspective. English Language Teaching, 10(4), 45-52. https://doi.org/10.5539/elt.v10n4p45

Shamsudin, D. S., Sadoughvanini, S., \& Hanafi Zaid, Y. (2013). Iranian EFL learners' collocational errors in speaking skill. Procedia - Social and Behavioral Sciences, 70, 1295-1302. https://doi.org/10.1016/j.sbspro.2013.01.190

Shannon-Baker, P. (2016). Making paradigms meaningful in mixed methods research. Journal of Mixed Methods Research, 10(4), 319-334. https://doi.org/10.1177/1558689815575861

Shariati, M. (2019). Students' attitudes towards the use of L1 in EFL classrooms: A case study at an English language school in Iran. Journal of Applied Linguistics and Language Research, 6(2), 198-217.

Shay, J. (2006). Reading Assessment and Instruction for All Learners. Guilford Press.

Sheybani, M. (2019). The relationship between EFL learners' willingness to communicate (WTC) and their teacher immediacy attributes: A structural equation modelling. Cogent Psychology, 6(1), 1607051. https://doi.org/10.1080/23311908.2019.1607051

Shin, S., Lee, J.-K., \& Ha, M. (2016). Influence of career motivation on science learning in Korean high-school students. Eurasia Journal of Mathematics, Science and Technology Education, 13(5), 1517-1538. https://doi.org/10.12973/eurasia.2017.00683a

Shuchi, I., \& Islam, A. B. M. S. (2016). Teachers' and students' attitudes towards L1 use in EFL classrooms in the contexts of Bangladesh and Saudi Arabia. English Language Teaching, 9(12), 62-73. https://doi.org/10.5539/elt.v9n12p62 
Shvidko, E. (2018). Learners' attitudes toward "English-Only" institutional policies: Language use outside the classroom. TESL Canada Journal, 34(2), 25-48. https://doi.org/1018806/tesl.v34i2.1261

Siggelkow, N. (2007). Persuasion with case studies. Academy of Management Journal, 50(1), 20-24. https://doi.org/10.5465/AMJ.2007.24160882

Signorini, P., Wiesemes, R., \& Murphy, R. (2009). Developing alternative frameworks for exploring intercultural learning: A critique of Hofstede's cultural difference model. Teaching in Higher Education, 14(3), 253-264. https://doi.org/10.1080/13562510902898825

Silva, C. M. V., Pereira, Í. S. P., \& Sebastião, I. (2019). Portuguese teachers' perceptions of grammar teaching. Crossroad - The Journal of English Studies, 24, 65-81. https://doi.org/10.15290/CR.2019.24.1.05

Simbolon, N. E., Oliver, R., \& Mercieca, P. (2020). Lecturers' perceptions of English medium instruction (EMI) practice at a university in Indonesia. Social Sciences \& Humanities, 28(2), 1065-1081.

Sinha, A., Banejee, N., Sinha, A., \& Shastri, R. (2009). Interference of first language in the acquisition of second language. Journal of Psychology and Counseling, 1(7), 117122.

Smit, K., de Brabander, C. J., \& Martens, R. L. (2014). Student-centred and teacher-centred learning environment in pre-vocational secondary education: Psychological needs, and motivation. Scandinavian Journal of Educational Research, 58(6), 695-712. https://doi.org/10.1080/00313831.2013.821090

Smotrova, T., \& Lantolf, J. P. (2013). The function of gesture in lexically focused L2 instructional conversations. The Modern Language Journal, 97(2), 397-416. https://doi.org/10.1111/j.1540-4781.2013.12008.x

Soleimani, N. (2018). EFL teachers' epistemological beliefs and classroom management approaches: A case study. The Journal of Language Teaching and Learning, 8(2), 4560 .

Song, B., \& Kim, T.-Y. (2016). Teacher (de)motivation from an Activity Theory perspective: Cases of two experienced EFL teachers in South Korea. System, 57, 134-145. https://doi.org/10.1016/j.system.2016.02.006

Song, W., Jin, X., Gao, J., \& Zhao, T. (2020). Will buying follow others ease their threat of death? An analysis of consumer data during the period of COVID-19 in China. International Journal of Environmental Research and Public Health, 17(9), 3215. https://doi.org/10.3390/ijerph17093215

Song, X., \& Dai, W. (2009). A study on input enhancement, output and noticing. Foreign Languages and Their Teaching, 10(2009), 5-9.

Song, Y., \& Andrews, S. (2009). The L1 in L2 learning: Teachers' beliefs and practices. LINCOM Europa. 
Sözer, M. A. (2019). Effective teacher immediacy behaviors based on students' perceptions. Universal Journal of Educational Research, 7(2), 387-393. https://doi.org/10.13189/ujer.2019.070211

Squires, A. (2008). Language barriers and qualitative nursing research: Methodological considerations. International Nursing Review, 55(3), 265-273. https://doi.org/10.1111/j.1466-7657.2008.00652.x

Stafford, C. A. (2012). What's on your mind? How private speech mediates cognition during initial non-primary language learning. Applied Linguistics, 34(2), 151-172. https://doi.org/10.1093/applin/ams039

Stake, R. E. (2005). Qualitative case studies. In N. K. Denzin \& Y. S. Lincoln ( $3^{\text {rd }}$ ed.), The Sage handbook of qualitative research (pp. 443-466). Sage.

Stake, R. E. (2010). Qualitative research: Studying how things work. Guilford Press.

Stefan, M. A., \& Popescu, A. M. (2020). Classroom communication in the online environment-perceptions and directions of action. Psychology-Pedagogy, 42, 161172.

Storch, N., \& Aldosari, A. (2010). Learners' use of first language (Arabic) in pair work in an EFL class. Language Teaching Research, 14(4), 355-375. https://doi.org/10.1177/1362168810375362

Strayhorn, T. L. (2018). College students' sense of belonging: A key to educational success for all students. Routledge.

Sugino, T. (2010). Teacher demotivational factors in the Japanese language teaching context. Procedia Social and Behavioral Sciences, 3, 216-226. https://doi.org/10.1016/j.sbspro.2010.07.036

Suharno, S. (2010). Cognitivism and its implication in the second language learning. Parole: Journal of Linguistics and Education, 1, 48-63.

Surkamp, C. (2014). Non-verbal communication: Why we need it in foreign language teaching and how we can foster it with drama activities. Scenario, 8(2), 12-27.

Suwaed, H. (2018). 'The choice is made from no choice': What motivates Libyan EFL teachers to work in times of change?. International Journal of Research, 7(4), 69-79. https://doi.org/10.5861/ijrsll.2018.3001

Swain, M. (2000). The output hypothesis and beyond: Mediating acquisition through collaborative dialogue. In J. P. Lantolf (Ed.), Sociocultural theory and second language learning (pp. 97-114). Oxford University Press.

Swain, M. (2013). The inseparability of cognition and emotion in second language learning. Language teaching, 46(2), 195-207. https://doi.org/10.1017/S0261444811000486

Swain, M., \& Deters, P. (2007). "New" mainstream SLA theory: Expanded and enriched. The Modern Language Journal, 91, 820-836. 
Swain, M., \& Lapkin, S. (1995). Problems in output and the cognitive processes they generate: A step towards second language learning. Applied Linguistics, 16(3), 371391.

Swain, M., \& Lapkin, S. (2013). A Vygotskian sociocultural perspective on immersion education: The L1/L2 debate. Journal of Immersion and Content-Based Language Education, 1(1), 101-129. https://doi.org/10.1075/jicb.1.1.05swa

Tan, C. (2017). Teacher beliefs on L1 use in multilingual classrooms: A New Zealand university language centre. New Zealand Society of Applied Linguistics, 23(1), 17-32.

Tariq, S., \& Woodman, J. (2013). Using mixed methods in health research. JRSM Short Reports, 4(6), 1-18. https://doi.org/10.1177/2042533313479197.

Tarone, E. (1977). Conscious communication strategies in interlanguage: A progress report. On TESOL, 77, 194-203.

Tarone, E. (1980). Communication strategies, foreigner talk, and repair in interlanguage. Language Learning, 30(2), 417-428.

Tarone, E., \& Swierzbin, B. (2009). Exploring learner language. Electronic Journal of Foreign Language Teaching, 8(2), 268-269.

Taşç1, S., \& Aksu Atac, B. (2020). L1 use in L2 teaching: The amount, functions, and perception towards the use of L1 in Turkish primary school context. Innovations in Education and Teaching International, 7(2), 655-667.

Tashakkori, A., \& Teddlie, C. (2016). SAGE handbook of mixed methods in social \& behavioral research (2nd ed.). Sage.

Tavakoli, Z., Rakhshanderoo, F., Izadpanah, M., \& Moradi, M. (2014). Ego identity types and language proficiency of Iranian EFL learners. Procedia-Social and Behavioral Sciences, 98, 1885-1894.

Tergujeff, E. (2013). Learner perspective on English pronunciation teaching in an EFL context. Research in Language, 11(1), 81-95.

Terry, G., Hayfield, N., Clarke, V., \& Braun, V. (2017). Thematic analysis. In C. Willig \& Stainton-Rogers (Eds.), The Sage handbook of qualitative research in psychology (2nd ed., pp. 17-37). Sage.

Thapa, A., Cohen, J., Guffey, S., \& Higgins-D’Alessandro, A. (2013). A review of school climate research. Review of Educational Research, 83(3), 357-385.

Thomas, L. (2012). Building student engagement and belonging in higher education at a time of change. Paul Hamlyn Foundation. https://www.heacademy.ac.uk/sites/default/files/resources/What_works_final_report.p $\underline{\text { df }}$

Thoms, J. J. (2012). Classroom discourse in foreign language classrooms: A review of the literature. Foreign Language Annals, 45(1), 8-27.

Thornberg, R., \& Charmaz, K. (2014). Grounded theory and theoretical coding. The SAGE handbook of qualitative data analysis, 5, 153-169. 
Thornton, J. (2017). Case study of English language teachers' perceptions of educating ELLs in English-only monolingual settings (Publication Number 10638682) [Doctoral dissertation, Northcentral University]. ProQuest Dissertations \& Theses Global.

Tobin, J., Hsueh, Y., \& Karasawa, M. (2009). Preschool in three cultures: China, Japan and the United States revisited. The University of Chicago Press.

Torrech, J. R. (2018). The role of human capital and innovative entrepreneurship in developing countries [Doctoral dissertation, Florida Institute of Technology, Melbourne, Florida]. http://hdl.handle.net/11141/2698

Tovar, E., \& Simon, M. (2010). Factorial structure and invariance analysis of the sense of belonging scales. Measurement and Evaluation in Counseling and Development, 43(3), 199-217. https://doi.org/10.1177/0748175610384811

Treiber, D. N. (2019). Is it who am I or who do you think I am?: Identity development of adolescents with substance use disorders (Publication Number 27534905) [Doctoral dissertation, Antioch University]. ProQuest Dissertations and Theses.

Trinh, T. T. H., \& Mai, T. L. (2019). Current challenges in the teaching of tertiary English in Vietnam. In J. Albright (Ed.), English Tertiary Education in Vietnam (1st ed., pp. 4053). Routledge. https://doi.org/helicon.vuw.ac.nz/10.4324/9781315212098

Tsegay, S. (2015). Students' experience in student-centered learning at higher education institutions in China: A case study. EDUCARE: International Journal for Educational Studies, 7(2), 135-146.

Tsiplakides, I., \& Areti, K. (2010). The relationship between teacher expectations and student achievement in the teaching of English as a foreign language. English Language Teaching, 3(2), 22-26. https://doi.org/10.5539/elt.v3n2p22

Tsukamoto, M. (2012). Students' perception of teachers' language use in an EFL classroom. 大阪女学院大学紀要 8 号, 8, 143-154.

Tunnisa, D. (2018, 12-14 July). Teachers' humor as a communication strategyin creating positive environment in English course [Paper presentation]. The 65th TEFLIN international conference, Indonesia.

Tziava, K. (2003). Factors that motivate and demotivate Greek EFL teachers [Master's dissertation, The University of Edinburgh]. Moray House Masters thesis collection. http://hdl.handle.net/1842/495

Uçar, H. 1. F., \& Soruç, A. (2018). Examining Turkish university students' sense of achievement, motivation, and anxiety: A comparison of the English-and Frenchmedium education systems. Eurasian Journal of Applied Linguistics, 4(2), 177-191.

Ünel, A., \& Mirioğlu, M. (2015). Syntactical modifications in teacher talk of native and nonnative speakers in EFL classrooms. Theory and Practice of Second Language Acquisition, 1(1), 125-141.

Uysal, D., \& Güven, M. (2018). Negative feelings of Turkish students in EFL learning process. European Journal of Foreign Language Teaching, 3(4), 120-140. https://doi.org/10.5281/zenodo.1469726 
Van Lier, L. (1996). Interaction in the language curriculum: Awareness, autonomy, and authenticity. Longman.

Van Lier, L. (1998). The relationship between consciousness, interaction and language learning. Language Awareness, 7(2-3), 128-145. https://doi.org/10.1080/09658419808667105

Vanpatten, B., \& Williams, J. (2014). Theories in second language acquisition: An introduction. Routledge.

Vietnamese Government. (2008). Decision No. 1400/QD-TTg Approval of the national project, Teaching and Learning Foreign Language in the Public-Sector Educational System for the 2008-2020 Period. Ha Noi.

http://vanban.chinhphu.vn/portal/page/portal/chinhphu/hethongvanban?class_id=1\&_ page $=1 \&$ mode $=$ detail\&document_id=78437

Vietnamese Government. (2010). Decision No. 3321/QĐ-BGDĐT, Approval of the program, Pilot English Teaching at Primary Schools. Hanoi. https://e-

ict.gov.vn/laws/detail/Quyet-dinh-ve-viec-ban-hanh-chuong-trinh-thi-diem-tiengAnh-tieu-hoc-29/

Vietnamese Government. (2017). Decision No. 2080/QĐ-TTg Approval of the adjustments and supplements of the national project, Teaching and Learning Foreign Language in the Public-Sector Educational System for the 2017-2025 Period. Hanoi. http://vanban.chinhphu.vn/portal/page/portal/chinhphu/hethongvanban?class_id=2\& page $=1 \&$ mode $=$ detail \&document_id=192343

Vietnamese Government. (2019). Circular No. 24/2019/TT-BGDDT The Training Regulations for Pre-service Teacher Education at the Intermediate and Tertiary Levels. Hanoi. https://moet.gov.vn/van-ban/vanban/Pages/chi-tietvanban.aspx?ItemID=1334

Villamil, O. S., \& De Guerrero, M. C. (1996). Peer revision in the L2 classroom: Socialcognitive activities, mediating strategies, and aspects of social behavior. Journal of second language writing, 5(1), 51-75.

Vu, N. T., \& Burns, A. (2014). English as a medium of instruction: Challenges for Vietnamese tertiary lecturers. Journal of Asia TEFL, 11(3), 1-31.

Vu, T. N. (2019). Theoretical constructs and practical strategies for intercultural communication: Informed teaching practices in Vietnam. Journal of Curriculum Studies Research, 1(1), 45-53.

$\mathrm{Vu}, \mathrm{T}$. T. N. (2017). The Vietnamese agenda of adopting English as a medium of instruction. VNU Journal of Foreign Studies, 33(3), 53-65.

Vu, V. D. (2017). An empirical study on negative transfer in Vietnamese tertiary EFL learners' English writing. The Journal of Teaching English for Specific and Academic Purposes, 5(4), 661-668. https://doi.org/10.22190/JTESAP1704661V

Vygotsky, L. S. (1978). Mind in society: The development of higher psychological processes. Harvard University Press. 
Vygotsky, L. S. (1997). The collected works of LS Vygotsky: Problems of the theory and history of psychology. Springer Science \& Business Media.

Wade, M. V. (2006). Likert-type scale response anchors. Clemson International Institute for Tourism \& Research Development, Department of Parks, Recreation and Tourism Management. Clemson University. Retrieved April 28 2017, from https://scholar.google.com/scholar?hl=en\&as_sdt=0\%2C5\&q=Likerttype+scale+response+anchors\&btnG $=$

Walsh, S. (2003). Developing interactional awareness in the second language classroom through teacher self-evaluation. Language Awareness, 12(2), 124-142. https://doi.org/10.1080/09658410308667071

Walsh, S. (2011). Exploring classroom discourse: Language in action. New York: Routledge.

Wang, Y. (2006). The factors in L2 Learning and Motivation and Their Pedagogical Implications in English Classroom. Sino-English Teaching, 3(7), 32-38.

Wang, Q., \& Castro, C. D. (2010). Classroom interaction and language output. English Language Teaching, 3(2), 175-186.

Wang, Y.-L. (2006). The factors in L2 learning motivation and their pedagogical implications in English classroom. Sino-US English Teaching, 3(7), 32-37.

Wei, D. (2013). The implementation of English-only policy in the tertiary EFL context in Taiwan. Indonesian Journal of Applied Linguistics, 2(2), 187-198. https://doi.org/10.17509/ijal.v2i2.164

Wei, M., Den Brok, P., \& Zhou, Y. (2009). Teacher interpersonal behaviour and student achievement in English as a foreign language classrooms in China. Learning Environments Research, 12(3), 157-174.

Wei, X. (2012). An Introduction to conversational interaction and second language acquisition. English Linguistics Research, 1(1), 111-117. https://doi.org/10.5430/elr.v1n1p111

Werani, A. (2018). Inner speech and its impact on teaching and learning. In J. P. Lantolf, M. E. Poehner, \& M. Swain (Eds.), The Routledge handbook of sociocultural theory and second language development (pp. 136-151). Routledge.

Widya, T., Fatimah, A. S., \& Santiana, S. (2020). Students'feedback as a tool for reflection: A narrative inquiry of an Indonesian pre-service teacher. Teaching and Learning English in Multicultural Contexts (TLEMC), 4(1), 1-11.

Willems, G. M. (1987). Communication strategies and their significance in foreign language teaching. System, 15(3), 351-364. https://doi.org/10.1016/0346-251X(87)90009-1

Woodside, A. G. (2010). Case study research theory, methods, practice (1st ed.). Emerald Group.

Wright, T. (2002). Doing language awareness: Issues for language study in language teacher education. In H. Trappes-Lomax \& G. Ferguson (Eds.), Language in language teacher education (pp. 113-130). John Benjamin. 
Yaghoubi-Notash, M., \& Karafkan, A. (2015). Teacher-employed communication strategies: Investigating function type occurrence in Iranian EFL context. International Journal of Applied Linguistics and English Literature, 4(2), 73-80.

https://doi.org/10.7575/aiac.ijalel.v.4n.2p.73

Yang, D., \& Gai, F. P. (2010). Chinese learners' communication strategies research: A case study at Shandong Jiaotong university. Cross-cultural Communication, 6(1), 56-81. https://doi.org/10.3968/j.ccc.1923670020100601.009

Yang, H. (2016). On the effective way of body language in the primary English teaching. 2nd International Conference on Economy, Management and Education Technology.

Retrieved October 17, 2019

https://scholar.google.com/scholar?hl=en\&as_sdt=0\%2C5\&q=\%22On+the+effective +way+of+body+language+in+the+primary+English+teaching $\% 22 \& b \operatorname{tnG}=$

Yang, Y. (2020, March). The application of experiential teaching method in nursing Specialty teaching. 4th International Conference on Culture, Education and Economic Development of Modern Society (ICCESE 2020) (pp. 1045-1047). Atlantis Press.

Yanti, G. S. (2019). Perceptions and attitudes of Indonesian teachers toward communicative language teaching. Jurnal Ilmiah Spectral, 5(1), 001-018.

Yashima, T. (2002). Willingness to communicate in a second language: The Japanese EFL context. The Modern Language Journal, 86(1), 54-66. https://doi.org/10.1111/1540$\underline{4781.00136}$

Yazdanmehr, E., Akbari, R., \& Kiany, G. (2020). Pedagogical content knowledge and expertise in ELT. Journal of English Language Teaching and Learning, 12(25), 324342.

Yen Nguyet. (2016, 21/11). Đề án Ngoại ngữ quốc gia 2020 thất bại, vì đâu? The world and Vietnam report. https://baoquocte.vn/de-an-ngoai-ngu-quoc-gia-2020-that-bai-vi-dau$\underline{39673 . h t m l}$

Yin, R. K. (2014). Case study research: Design and methods (5th ed.). Sage.

Yule, G., \& Tarone, E. (1991). The other side of the page: Integrating the study of communication strategies and negotiated input in SLA. Foreign/Second Language Pedagogy Research, 142-161.

Yuvayapan, F. (2019). Translanguaging in EFL classrooms: Teachers' perceptions and practices. Journal of Language and Linguistic Studies, 15(2), 678-694. https://doi.org/10.17263/jlls.586811

Zemel, A., \& Koschmann, T. (2011). Pursuing a question: Reinitiating IRE sequences as a method of instruction. Journal of Pragmatics, 43(2), 475-488. https://doi.org/10.1016/j.pragma.2010.08.022

Zhang, Q. (2019). Construction of teacher scaffolding model in college English teaching. 2019 [Paper presentation]. International Conference on Reform, Technology, Psychology in Education, UK. Retrieved September 6, 2019, from https://webofproceedings.org/proceedings_series/ESSP/ICRTPE\%202019/ICRTPE16 6.pdf 
Zhou, W., \& Li, G. (2015). Chinese language teachers' expectations and perceptions of American students' behavior: Exploring the nexus of cultural differences and classroom management. System, 49(2015), 17-27.

https://doi.org/10.1016/j.system.2014.10.011 


\section{Appendix A: Ethic approval letter}

\section{Appendices}

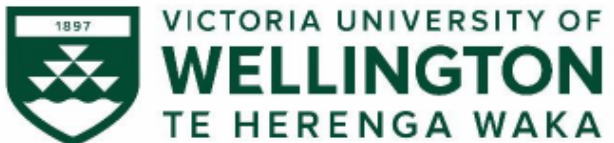

\begin{tabular}{l|l}
\hline TO & Lien Tran \\
\hline FROM & $\begin{array}{l}\text { Associate Professor Judith Loveridge, Convenor, Human Ethics } \\
\text { Committee }\end{array}$ \\
\hline
\end{tabular}

\begin{tabular}{l|l}
\hline DATE & 1 December 2017 \\
\hline PAGES & 1 \\
\hline
\end{tabular}

\begin{tabular}{l|l}
\hline SUBJECT & Ethics Approval \\
& Number: 25461 \\
& Title: Vietnamese teachers' oral English communication strategies \\
& in non-English major classes at tertiary levels - A multiple-case \\
& study in Danang city
\end{tabular}

Thank you for your application for ethical approval, which has now been considered by the Human Ethics Committee.

Your application has been approved from the above date and this approval is valid for three years. If your data collection is not completed by this date you should apply to the Human Ethics Committee for an extension to this approval.

Best wishes with the research

Kind regards,

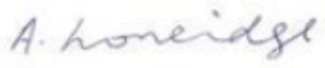

Judith Loveridge

Convenor, Victoria University of Wellington Human Ethics Committee 


\section{Appendix B: Consent forms}

TE WHARE WĀNANGA O TE ŪPOKO O TE IKA A MĀUI

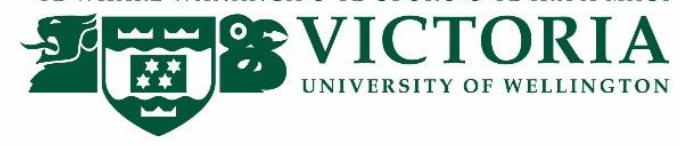

\section{VIETNAMESE TERTIARY TEACHERS' ENGLISH COMMUNICATION IN NON-ENGLISH MAJOR CLASSES - A CASE STUDY}

\section{THE PRINCIPAL/FACULTY DEAN CONSENT TO CONDUCT RESEARCH}

This consent form will be held for five years.

Researcher: $\quad$ Lien Tran Thi Thuy, School of Education, Victoria University of Wellington.

* I have read the Information Sheet and the project has been explained to me. My questions have been answered to my satisfaction. I understand that I can ask further questions at any time.

* I permit the researcher to conduct her study with lecturers and students at the college/faculty. I understand that:

* I may withdraw permission for this study to take place at my university at any point before $\mathbf{1 5}^{\text {th }}$ March 2018, without giving any reason.

* Any information participants provide will be kept confidential to the researcher and her supervisors. I understand that the results will be used for a $\mathrm{PhD}$ report and a summary of the results may be used in academic reports and/or publications, and/or presented at conferences.

* My college name will not be used in reports, nor will any information that would identify my college/faculty.

* I would like to receive a summary of the findings of the thesis and $\quad * \quad$ Yes $\square \quad$ No $\square$ have added my email address below.

Signature of the Principal/ Faculty Dean:

Name of the Principal/ Faculty Dean:

Date:

Email address: 


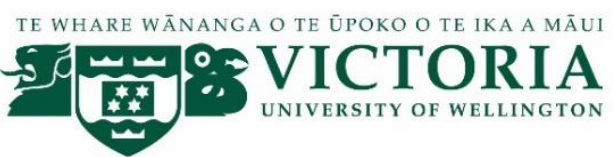

\section{VIETNAMESE TERTIARY TEACHERS' ENGLISH COMMUNICATION IN NON-ENGLISH MAJOR CLASSES - A CASE STUDY}

\section{LECTURER CONSENT FOR OBSERVATIONS, QUESTIONNAIRE, AND INTERVIEW}

This consent form will be held for five years.

Researcher: $\quad$ Lien Tran Thi Thuy, School of Education, Victoria University of Wellington.

* I have read the Information Sheet and the project has been explained to me. My questions have been answered to my satisfaction. I understand that I can ask further questions at any time. I know that agreeing this consent means I agree to:

* have between two and four classroom observations conducted in different classes of mine;

* have all my observed lessons audio-recorded;

* participate in a paper-based questionnaire survey;

* take part in an audio-recorded interview.

I understand that:

* I may withdraw from this study at any point before $15^{\text {th }}$ March 2018, without giving any reason, and any information that I have provided will be destroyed.

* I can choose not to answer any question(s) in the questionnaire and interview.

* I can ask for the audio-recording device to be turned off at any time during the observations.

* I can ask for the audio recorder to be turned off at any time during the interview.

* Any information I provide will be kept confidential to the researcher and her supervisors. I understand that the results will be used for a PhD report and a summary of the results may be used in academic reports and/or publications and/or presented at conferences.

* The information I have provided will be destroyed five years after the data gathering is finished.

* My name will not be used in reports, nor will any information that would identify me.

* I would like a summary of the transcript of my interview.

* Yes

No

* I would like to receive a summary of findings of the thesis and

* Yes

No have added my email address below.

Signature of lecturer:

Name of lecturer:

Date:

Email address: 


\section{VIETNAMESE TERTIARY TEACHERS' ENGLISH COMMUNICATION IN NON-ENGLISH MAJOR CLASSES - A CASE STUDY \\ STUDENT CONSENT FOR OBSERVATIONS AND QUESTIONNAIRE}

This consent form will be held for five years.

Researcher: $\quad$ Lien Tran Thi Thuy, School of Education, Victoria University of Wellington.

* I have read the Information Sheet and the project has been explained to me. My questions have been answered to my satisfaction. I understand that I can ask further questions at any time. I know that agreeing this consent means I agree to:

* take part in two classroom observations;

* have my observed lessons audio-recorded;

* take part in a questionnaire survey.

I understand that:

* I may withdraw from this study at any point before $15^{\text {th }}$ March 2018, without giving any reasons, and any information that I have provided will be destroyed.

* I can choose not to answer any question(s) in the questionnaire.

* Any information I provide will be kept confidential to the researcher and her supervisors. I understand that the results will be used for a $\mathrm{PhD}$ report and a summary of the results may be used in academic reports and/or presented at conferences.

* The information I have provided will be destroyed five years after the data gathering is finished.

* My name will not be used in reports and/or publications, nor will any information that would identify me.

Signature of participant:

Name of participant:

Date:

Email address: 


\section{VIETNAMESE TERTIARY TEACHERS' ENGLISH COMMUNICATION IN NON-ENGLISH MAJOR CLASSES - A CASE STUDY}

\section{STUDENT CONSENT FOR FOCUS GROUP INTERVIEW}

This consent form will be held for five years.

Researcher: $\quad$ Lien Tran Thi Thuy, School of Education, Victoria University of Wellington.

* I have read the Information Sheet and the project has been explained to me. My questions have been answered to my satisfaction. I understand that I can ask further questions at any time.

* I agree to take part in an audio-recorded focus group interview.

* I agree to follow the protocol for the focus group interview.

I understand that:

* It is not possible to withdraw information from a focus group once it has commenced, as it is usually part of discussion with other participants. Therefore, I may withdraw from this study at any point before the commencement of the focus group interview, without giving any reasons, and any information that I have provided will be destroyed.

* I can choose not to answer any question(s) in the interview.

* Any information I provide will be kept confidential to the researcher and her supervisors. I understand that the results will be used for a PhD report and a summary of the results may be used in academic reports and/or publications and/or presented at conferences.

* The information I have provided will be destroyed five years after the data gathering is finished.

* My name will not be used in academic reports and/or publications, nor will any information that would identify me.

* I would like to receive a summary of my interview and have added

Yes No $\square$ my email address below.

Signature of participant:

Name of participant:

Date:

Email address: 


\section{Appendix C: Research information sheet}

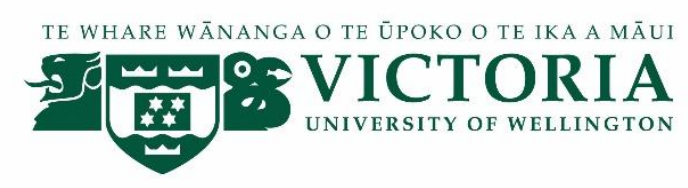

\section{INFORMATION SHEET FOR THE PRINCIPAL/FACULTY DEAN}

Dear

Project title:

\section{VIETNAMESE TERTIARY TEACHERS' ENGLISH COMMUNICATION IN NON-ENGLISH MAJOR CLASSES - A CASE STUDY}

Thank you for your interest in this project. Please read this information before deciding whether or not to give permission. If you decide to give permission, thank you. If you decide not to give permission, thank you for considering my request.

\section{Who am I?}

I am Lien Tran Thi Thuy and currently a Doctoral candidate at Victoria University of Wellington. This research project is work towards my $\mathrm{PhD}$ thesis.

\section{What is the aim of the project?}

This thesis aims to find out about lecturers use of English as a medium of teaching and classroom-based communication with non-English major classes. The findings of this study are intended to lead to suggestions for English learning and teaching for further improvement of students' speaking and listening skills. The findings may also inform policies of English education in other education contexts. This research has been approved by the Victoria University of Wellington Human Ethics Committee [Approval number: 0000025461].

\section{What am I going to do?}

If you agree to give permission for my study to be conducted in your college, I will invite lecturers and students to three types of the data gathering. I am intending to:

- observe between two and four lessons of each lecturer who agrees to participate. I will make observation notes about how English is being used during the lessons. I will also audio-record the observed lessons for later review.

- invite those lecturers and all students in the observed classes to complete paper-based questionnaire surveys. The questionnaires for the lecturers investigate what they perceive to be enablers and hindrances to using English in teaching and communication in non-English major 
classes. The students' questionnaire explores their perceptions on things that help and hinder their comprehending their lecturers' spoken English. Each questionnaire will take up to half an hour to complete.

- interview those lecturers about the use of spoken English in non-English major classes. The interview will take up to one hour. I will also interview groups of six students (one group for each participating lecturer) about their English language learning experiences. The group interviews for students will take up to one hour and a half. I will audio-record the interviews and transcribe them later.

You can withdraw your permission by contacting me at any point before $15^{\text {th }}$ March 2018 . If you withdraw, the information collected from the lesson observations, questionnaires, and interviews from the lecturers and students will be destroyed or returned to them.

\section{What will happen to the information collected from your college?}

Throughout the project, all attempts will be made to minimise the disruptive impact on teaching and learning activities of your lecturers and students. This research is confidential. The research data will be aggregated and your college identity will not be disclosed in any reports, presentations, or public documentation.

\section{What will the project produce?}

The information from my research will be used in my $\mathrm{PhD}$ dissertation, written publications and oral presentations in national and international contexts.

\section{If you accept this invitation, what are your rights as a research participant?}

You do not have to accept this invitation if you don't want to. If you do decide to permit me to conduct this study at your college, you have the right to ask any questions about the study at any time and withdraw the consent from the study before $15^{\text {th }}$ March 2018 .

\section{If you have any questions or problems, who can you contact?}

If you have any questions, either now or in the future, please feel free to contact either:

\section{Student:}

Name: Lien Tran Thi Thuy

University email address:

Lien.Tran@vuw.ac.nz

\section{Supervisors:}

Name: Assoc. Prof. Jenny Ritchie

Role: Doctoral supervisor

School: Education

Phone: +6444635716

Jenny.Ritchie@vuw.ac.nz 
Name: Dr. Jae Major

Role: Doctoral supervisor

School: Education

Phone: +6444639508

Jae.Major@vuw.ac.nz

\section{Human Ethics Committee information}

If you have any concerns about the ethical conduct of the research, you may contact the Victoria University HEC Convener: Associate Professor Susan Corbett. Email Susan.corbett@ vuw.ac.nz or telephone $+64-4-4635480$. 


\section{INFORMATION SHEET FOR LECTURER}

\section{Dear}

Project title:

\section{VIETNAMESE TERTIARY TEACHERS' ENGLISH COMMUNICATION IN NON-ENGLISH MAJOR CLASSES - A CASE STUDY}

Thank you for your interest in this project. Please read this information before deciding whether or not to take part. If you decide to participate, thank you. If you decide not to take part, thank you for considering my request.

\section{Who am I?}

I am Lien Tran Thi Thuy and currently a Doctoral candidate at Victoria University of Wellington. This research project is work towards my $\mathrm{PhD}$ thesis.

\section{What is the aim of the project?}

This thesis aims to find out about the English that lecturers use as a medium of teaching and classroom-based communication with non-English major students. The findings of this study are intended to lead to suggestions for English learning and teaching for further improvement of students' speaking and listening competence. The findings may also inform policies of English education in other education contexts. This research has been approved by the Victoria University of Wellington Human Ethics Committee [Approval number: 0000025461] and permitted by the Principal and the Faculty Dean. Data collected from you will be used for a research project and not for evaluation of your performance as an employee.

\section{How can you help?}

If you agree to take part, you will be involved in up to three types of the data gathering. I am intending to:

- observe between two and four lessons of your usual teaching. It is your decision what classes and lessons will be observed, based on the provided criteria, at any time during my field trip at your college (from February 11 to March 29, 2018). 5 minutes before each observation, a small audio-recorder will be fixed in the middle of the classroom and I will make sure that it will not affect the students. To better record your voice, you will also be wearing a small audio recording 
device. I will be in the back of the class to avoid students' attention. I will not speak to any student and will try to minimise any disruption during my observations. I will make observation notes about how you and your students are using English during the lessons.

- invite you to complete a paper-based questionnaire survey which investigates what you perceive to be enablers and hindrances to using English in teaching and communication in nonEnglish major classes. The questionnaire will take up to half an hour to complete.

- interview you about the use of spoken English in non-English major classes. The interview will take up to one hour. I will record the interview and write it up later.

You can withdraw from the study by contacting me at any point before $15^{\text {th }}$ March 2018. If you withdraw, the information you provided from the classroom observations, questionnaire and individual interview will be destroyed or returned to you.

\section{What will happen to the information you give?}

Throughout the project, all attempts will be made to minimise the disruptive impact on your teaching and your students' learning activities. This research is confidential. The research data will be aggregated and your identity will not be disclosed in any reports, presentations, or public documentation. The observation fieldnotes, audio transcripts, questionnaire feedback, interview transcripts, summaries and any recordings will be kept securely and destroyed five years after the data gathering ends.

\section{What will the project produce?}

The information from my research will be used in my $\mathrm{PhD}$ dissertation, written publications and oral presentations in national and international contexts.

If you accept this invitation, what are your rights as a research participant?

You do not have to accept this invitation if you don't want to. If you do decide to participate, you have the right to:

- ask any questions about the study at any time;

- $\quad$ ask for the audio recording to be turned off at any time during the observations;

- choose not to answer any question(s) in the questionnaire and interview;

- $\quad$ ask for the audio recorder to be turned off at any time during the interview;

- read over and comment on a written summary of your interview;

- be able to read any reports of this research by emailing the researcher to request a copy.

You will be given a consent form to complete before the data gathering begins.

\section{If you have any questions or problems, who can you contact?}

If you have any questions, either now or in the future, please feel free to contact either:

\section{Student:}

Name: Lien Tran Thi Thuy

University email address:

Lien.Tran@vuw.ac.nz

\section{Supervisors:}

Name: Assoc. Prof. Jenny Ritchie

Role: Doctoral supervisor

School: Education 
Phone: +6444635716

Jenny.Ritchie@vuw.ac.nz

Name: Dr. Jae Major

Role: Doctoral supervisor

School: Education

Phone: +6444639508

Jae.Major@vuw.ac.nz

\section{Human Ethics Committee information}

If you have any concerns about the ethical conduct of the research you may contact the Victoria University HEC Convener: Associate Professor Susan Corbett. Email Susan.corbett@ vuw.ac.nz or telephone $+64-4-4635480$. 


\section{INFORMATION SHEET FOR STUDENT}

Project title:

\section{VIETNAMESE TERTIARY TEACHERS' ENGLISH COMMUNICATION IN NON-ENGLISH MAJOR CLASSES - A CASE STUDY}

Thank you for your interest in this project. Please read this information before deciding whether or not to take part. If you decide to participate, thank you. If you decide not to take part, thank you for considering my request. Remember that whether you will take part or not will not affect your learning scores or results at school. This research has been permitted by the Principal, the faculty Dean, and the lecturer.

\section{Who am I?}

I am Lien Tran Thi Thuy and currently a Doctoral candidate at Victoria University of Wellington. This research project is work towards my thesis.

\section{What am I doing? Why am I doing this?}

I am doing a research which aims to find out about the English that lecturers use as a medium of teaching and classroom-based communication with non-English major students. I will watch two of your lessons. I will make notes about how your lecturer and you are using spoken English in class. I will be at the back of the classroom and will not talk with you or interrupt the lessons. I will audiorecord your lessons so that I can listen again later.

I want to make good suggestions for further improvement of the teaching of English speaking and listening. Findings of this study may also inform policies of English education.

\section{How can you help?}

If you agree to take part you will be involved in up to three types of the data gathering. I am intending to:

- observe about two of your lessons. I will make observation notes and audio-record your lessons for later review.

- invite you to complete a paper-based questionnaire survey which investigates what you perceive helps and hinders your comprehension of your lecturers' spoken English in class. The questionnaire will take up to half an hour.

- interview you (if you desire) together with some other students about your ideas on your lecturers' spoken English in your lessons. The group interview will take up to one hour and a half. I will record the interview and write it up later. 
You can withdraw from the study by contacting me at any point before $15^{\text {th }}$ March 2018 . If you withdraw, the information you provided from the questionnaire and group interview will be destroyed or returned to you.

\section{What will happen to the information I collect?}

This research is confidential. All the data I gather will be put together and individual people will not be identified.

If you accept this invitation, what are your rights as a research participant?

You do not have to accept this invitation if you don't want to. If you do decide to participate, you have the right to:

- $\quad$ ask any question(s) about the study at any time;

- choose not to answer any question(s) in the questionnaire and interview;

- withdraw from the study up to the commencement of the focus group interview;

- read over and comment on a written summary of the interview.

You will be given a consent form to complete before the data gathering begins.

If you have any questions or problems, who can you contact?

If you have any questions, either now or in the future, please feel free to contact either:

\section{Student:}

Name: Lien Tran Thi Thuy

University email address:

Lien.Tran@ vuw.ac.nz

\section{Supervisors:}

Name: Assoc. Prof. Jenny Ritchie

Role: Doctoral supervisor

School: Education

Phone: +6444635716

Jenny.Ritchie@vuw.ac.nz

Name: Dr. Jae Major

Role: Doctoral supervisor

School: Education

Phone: +6444639508

Jae.Major@vuw.ac.nz

\section{Human Ethics Committee information}

If you have any concerns about the ethical conduct of the research you may contact the Victoria University HEC Convener: Associate Professor Susan Corbett. Email Susan.corbett@vuw.ac.nz or telephone +64-4-463 5480 . 
Appendix D: Data collection procedures

\begin{tabular}{|c|c|c|c|c|c|}
\hline Stages & $\begin{array}{c}\text { Researcher's } \\
\text { role }\end{array}$ & Time & Participants & $\begin{array}{l}\text { Aims and } \\
\text { research } \\
\text { questions }\end{array}$ & Techniques \\
\hline $\begin{array}{l}\text { 1. Gaining } \\
\text { ethnic } \\
\text { approval }\end{array}$ & Applicant & $\begin{array}{l}\text { October } \\
2017\end{array}$ & & & \\
\hline $\begin{array}{l}\text { 2. Piloting } \\
\text { the } \\
\text { instruments }\end{array}$ & $\begin{array}{l}\text { Deliverer } \\
\text { Collector } \\
\text { Interviewer }\end{array}$ & $\begin{array}{l}\text { November } \\
\text { 2017-January } \\
2018\end{array}$ & $\begin{array}{l}\text { Two evening } \\
\text { English } \\
\text { classes in } \\
\text { New Zealand } \\
\text { (one teacher; } \\
28 \text { learners) } \\
\text { One EFL } \\
\text { class at a } \\
\text { college in Da } \\
\text { Nang city } \\
\text { (one lecturer; } \\
25 \text { students) }\end{array}$ & $\begin{array}{l}\text {-Checking if there } \\
\text { is something wrong } \\
\text { with data gathering } \\
\text { tools } \\
\text {-Making suitable } \\
\text { modifications in } \\
\text { instruments }\end{array}$ & $\begin{array}{l}\text {-Audio- } \\
\text { recording } \\
\text {-Fieldnotes }\end{array}$ \\
\hline $\begin{array}{l}3 . \\
\text { Gaining } \\
\text { permission } \\
\text { from } \\
\text { authorities } \\
\text { and emailing } \\
\text { invitations }\end{array}$ & Sender & January 2018 & $\begin{array}{l}\text {-Head of } \\
\text { colleges } \\
\text {-Deans of } \\
\text { English } \\
\text { departments } \\
\text {-Six lecturers } \\
\text { (Ls) }\end{array}$ & $\begin{array}{l}\text {-Ask for colleges' } \\
\text { permission to carry } \\
\text { out the research in } \\
\text { real settings } \\
\text {-Ask for Ls' } \\
\text { agreement to make } \\
\text { observations, } \\
\text { video-recordings, } \\
\text { questionnaires and } \\
\text { interviews }\end{array}$ & \\
\hline $\begin{array}{l}4 . \\
\text { Classroom } \\
\text { observation }\end{array}$ & $\begin{array}{l}\text { Non- } \\
\text { participant } \\
\text { observer }\end{array}$ & $\begin{array}{l}\text { February } \\
2018 \text { (Mid- } \\
\text { term) } \\
45 \text { mins (first } \\
\text { five mins to } \\
\text { get tools } \\
\text { prepared) }\end{array}$ & $\begin{array}{l}\text {-Ten classes } \\
\text { of non- } \\
\text { English } \\
\text { major (two } \\
\text { classes for } \\
\text { each lecturer; } \\
\text { at least three } \\
\text { lessons for } \\
\text { each class) } \\
\text {-Five Ls }\end{array}$ & $\begin{array}{l}\text { Obtaining data in } \\
\text { real practices to } \\
\text { address research } \\
\text { questions } 1,2\end{array}$ & $\begin{array}{l}\text {-Audio- } \\
\text { recording } \\
\text {-Fieldnotes }\end{array}$ \\
\hline $\begin{array}{l}\text { 5. Structured } \\
\text { Questionnair } \\
\text { es }\end{array}$ & $\begin{array}{l}\text { Deliverer } \\
\text { and collector }\end{array}$ & $\begin{array}{l}\text { March } 2018 \\
\text { On the last } \\
\text { observation } \\
\text { day }\end{array}$ & $\begin{array}{l}\text {-All Ss from } \\
10 \text { classes } \\
\text { ( } 289 \\
\text { students) }\end{array}$ & $\begin{array}{l}\text { Collecting data } \\
\text { from students' (Ss) } \\
\text { perspectives and } \\
\text { addressing research } \\
\text { question } 4\end{array}$ & Paper-based \\
\hline $\begin{array}{l}\text { 6. Focus } \\
\text { group } \\
\text { interview }\end{array}$ & Moderator & $\begin{array}{l}\text { March } 2018 \\
\text { One week } \\
\text { after the } \\
\text { questionnaire } \\
\text { s (From an } \\
\text { hour to half } \\
\text { an hour for } \\
\text { each } \\
\text { interview) }\end{array}$ & $\begin{array}{l}\text {-Five groups } \\
\text { of Ss from } \\
\text { twelve } \\
\text { classes ( } 21 \\
\text { students) }\end{array}$ & $\begin{array}{l}\text { Collecting data } \\
\text { from Ss' } \\
\text { perspectives and } \\
\text { addressing research } \\
\text { question } 1,2 \text {, and } 4\end{array}$ & $\begin{array}{l}\text { Audio- } \\
\text { recording }\end{array}$ \\
\hline
\end{tabular}




\begin{tabular}{|l|l|l|l|l|l|}
\hline $\begin{array}{l}\text { 7. structured } \\
\text { questionnair }\end{array}$ & $\begin{array}{l}\text { Deliverer } \\
\text { Collector }\end{array}$ & $\begin{array}{l}\text { March 2018 } \\
- \text { On the last } \\
\text { observation } \\
\text { day }\end{array}$ & Five Ls & $\begin{array}{l}\text { Collect data from } \\
\text { Ls' perspectives } \\
\text { and address } \\
\text { research questions } \\
1,2 \text { and 3 }\end{array}$ & Paper-based \\
\hline $\begin{array}{l}\text { 8. In-depth } \\
\text { semi- } \\
\text { structured } \\
\text { interviews }\end{array}$ & Interviewer & $\begin{array}{l}\text { March 2018 } \\
\text { - One week } \\
\text { after the } \\
\text { questionnaire } \\
\text { s } \\
\text { (From 45 } \\
\text { minutes to } \\
\text { one hour for } \\
\text { each } \\
\text { interview) }\end{array}$ & Five Ls & $\begin{array}{l}\text { - Obtain data from } \\
\text { perspective of Ls } \\
\text { to address research } \\
\text { questions 1, 2 and } \\
3\end{array}$ & $\begin{array}{l}\text { Audio- } \\
\text { recording }\end{array}$ \\
& & & & & \\
& & & & & \\
& & & & \\
& & & & \\
\end{tabular}




\section{Appendix E: Survey questions for selecting lecturer participants}

Please write down your personal information. All the information you provide in the questionnaires will be confidential.

Code: / Year of birth:

Gender: $\quad \square$ Male $\square$ Female

Directions: This short questionnaire is intended to find out if you are going to be a suitable participant in this study, which aims at investigating how lecturers of English use English oral communication in non-English major classes. Please answer the questions carefully and give me feedback within 3 days. You are going to be informed whether or not to be invited for the study and described the whole research in detail then.

1. Are you holding a Bachelor's/Master's degree in Linguistics or/and the English language?

$$
\text { Yes } \square \quad N o \square
$$

2. Have you been teaching English for non-English major students for more than 5 years?

$$
\text { Yes } \square \quad N o \square
$$

3. How much are you interested in using oral English communication? (Please both tick the option and state the percentage of interest)

$0 \%$

$100 \%$

Not at all $\square \quad$ Slightly $\square \quad$ Moderately $\square \quad$ Very much $\square \quad$ Extremely $\square$ 


\section{Appendix F: Classroom observation protocol}

\section{CLASSROOM OBSERVATION PROTOCOL}

Research title: VIETNAMESE TERTIARY TEACHERS' ENGLISH COMMUNICATION

\section{IN NON-ENGLISH MAJOR CLASSES - A CASE STUDY}

School's code: Lecturer's code:

Name of class: Level of class:

Content(s)/skill(s) of lesson: 1

Main major of student attendants:

Number of student attendants:

Date of observation/recording:

Time In: Time Out:

Purpose: To record behaviours of the lecturer relating to his/her using English as a medium of teaching and classroom-based communication in non-English major classes.

Notes: Coding is only applied for three categories: Strategies, Purposes, and Frequency

Observation note-taking (on laptop)

\begin{tabular}{|c|c|c|c|c|c|}
\hline Time & $\begin{array}{l}\text { Lecturers' } \\
\text { speech }\end{array}$ & \begin{tabular}{l}
\multicolumn{1}{c}{ Strategies } \\
Code-switching (CS) \\
Translation (T) \\
Repetition (R) \\
Interaction (I) \\
Nonverbal (NV) \\
Simplification (S) \\
Other (O) (note down)
\end{tabular} & Purposes & $\begin{array}{l}\text { Students' } \\
\text { reactions }\end{array}$ & Other notes \\
\hline & & & & & \\
\hline & & & & & \\
\hline
\end{tabular}

\section{Overall Look}

1. Did lesson content allow for the teacher's speaking a lot of English? Yes/ No

2. How did the teacher interact with students when she was speaking English?

$$
1 \text { to } 1 \square \quad \text { with groups } \square \quad \text { with all/ nearly all class } \square
$$

3. How much did individuals engage in the teacher when she was speaking English to them?
Not at all $\square \quad$ Slightly $\square \quad$ Moderately $\square$
Very $\square$
Extremely $\square$

4. How much did all/nearly all class engage in the teacher when she was speaking English to them?

Not at all $\square \quad$ Slightly $\square \quad$ Moderately $\square \quad$ Very $\square \quad$ Extremely $\square$ 


\section{Appendix G: Questionnaire for lecturers}

\section{QUESTIONNAIRE FOR LECTURERS}

Directions: This questionnaire is intended to gather your views about what encourages and discourages your use of English as a medium of teaching and communication in non-English major classes. Please answer each question and return it to Ms. Lien as soon as possible.

Notes: The students mentioned here mean all those who you have been teaching English at the college.

Please write down your personal information. All the information you provide in the questionnaires will be confidential.

Name: / Year of birth:

Degrees/Qualifications:

Gender: $\quad \square$ Male $\quad \square$ Female

Years of teaching English to non-English major students

$$
\square \text { 5-10 } \square \text { 11-15 } \square \text { Over } 15
$$

\section{Section 1: General information}

1. In general, how much do you speak English in lessons (apart from speaking lessons) in non-English major classes? (Tick one only)

$\square$ Not at all $\quad \square$ Slightly $\quad \square$ Moderately $\quad \square$ Very much $\quad \square$ All the time

2. In general, to what extent are you satisfied with your use of oral English in non-English major classes? (Tick one only)

$\square$ Not at all $\square$ Slightly $\square$ Moderately $\square$ Very much $\square$ Extremely

3. In general, to what extent do you find it difficult to use oral English as the medium of teaching and communication in non-major classes? (Tick one only)

$\square$ Not at all $\square$ Slightly $\square$ Moderately $\square$ Very much $\quad \square$ Extremely

4. How do you describe the attitude of the majority of your non-English major students when you speak English to them? (Tick one only)

$\square$ Very negative $\square$ Slightly negative $\square$ Neutral $\square$ Slightly positive $\square$ Very positive

4'. If you chose "Very negative" or "Slightly negative", what are possible reasons from your perspective? (Tick all that apply)

$\square$ a. It goes beyond their level of understanding

$\square$ b. They find it unnecessary because they are non-English majors

$\square$ c. They do not try to concentrate on it

$\square$ d. Other reason(s):

4". If you chose "Very positive" or "Slightly positive", what are possible reasons from your perspective? (Tick all that apply)

$\square$ a. It matches their level of understanding

$\square$ b. They find it necessary for the improvement of their listening skill

$\square$ c. They find it necessary for their general communication outputs

$\square$ d. Other reason(s): 
5. To what extent do you think your English speaking in classes has positive effect on non-English major students' learning attitude? (Tick one only)

$\square$ Not at all $\square$ Slightly $\square$ Moderately $\quad \square$ Very much $\quad \square$ Extremely

5'. If you chose "Not at all" or "Slightly", what are possible reasons from your perspective? (Tick all that apply)

$\square$ a. It goes beyond their level of understanding

$\square$ b. They do not try to concentrate on it

$\square$ c. The strategies I am using may be not suitable

$\square$ d. I find it difficult to make them understand

$\square$ e. Other reason(s):

5". If you chose "Very much" or "Extremely", what are possible reasons from your perspective? (Tick all that apply)

$\square$ a. It matches their level of understanding

$\square$ b. The students try to concentrate on it

$\square$ c. The strategies I am using may be suitable

$\square$ d. I try hard to make them understand

$\square$ e. Other reason(s):

Section 2: My use of English as the medium of teaching and communication in non-English major classes

6. How much do you think the majority of your non-English major students understand your oral English? (Tick one only)

$\square$ Not at all $\square$ Slightly $\square$ Moderately $\square$ Very much $\square$ Extremely well

7. How do you feel and what do you do when your non-English major students always use Vietnamese to respond to your English questions/ requirements? (Tick one only)

$\square$ a. Angry (Your reaction:

b. Demotivated (Your reaction:

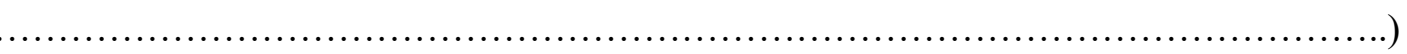

$\square$ c. Neutral (Your reaction:

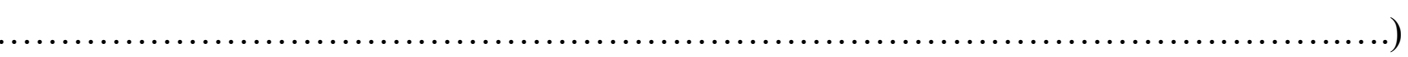

$\square$ d. Other(s):

(Your reaction:

8. What makes you motivated when speaking English in non-English major classes? (Tick all that apply)

$\square$ a. The students are always eager to listen 
$\square$ b. The students are engaged in responding to my speaking

$\square$ c. It is a chance to maintain my English speaking skill

$\square$ d. This creates a real English atmosphere and environment

$\square$ e. Other(s):

9. What makes you demotivated when speaking English in non-major classrooms? (Tick all that apply)

$\square$ a. The students' inability to understand it

$\square$ b. The students' negative attitude (e.g. not concentrating/ not being eager/ not being engaged ...)

$\square \mathrm{c}$. The constant code-switching between Vietnamese and English

$\square$ d. The constant translation between Vietnamese and English

口 e. Other(s):

10. What teaching strategies do you find it easiest to use when speaking English in non-English major classes? (Tick one only)
$\square$ a. Gestures
$\square$ b. Demonstration
$\square$ c. Simplification
$\square$ d. Translation
$\square$ e. Interaction
$\square$ f. Other(s):

11. What teaching strategies do you find it most difficult to use when speaking English in non-English major classes? (Tick one only)
$\square$ a. Gestures
$\square$ b. Demonstration
$\square$ c. Simplification
$\square$ d. Translation
$\square$ e. Interaction
$\square$ f. Other(s):

12. Following are some objective aspects that may be barriers to your use of oral English in non-English major classes. Read each aspect and label them from 1 to 5, using the rating levels:

\section{1 - Not a barrier at all}

\section{2 - Somewhat of a barrier}

\section{3 - Moderate barrier}

\section{4 - Somewhat strong barrier}

\section{5 - Extreme barrier}

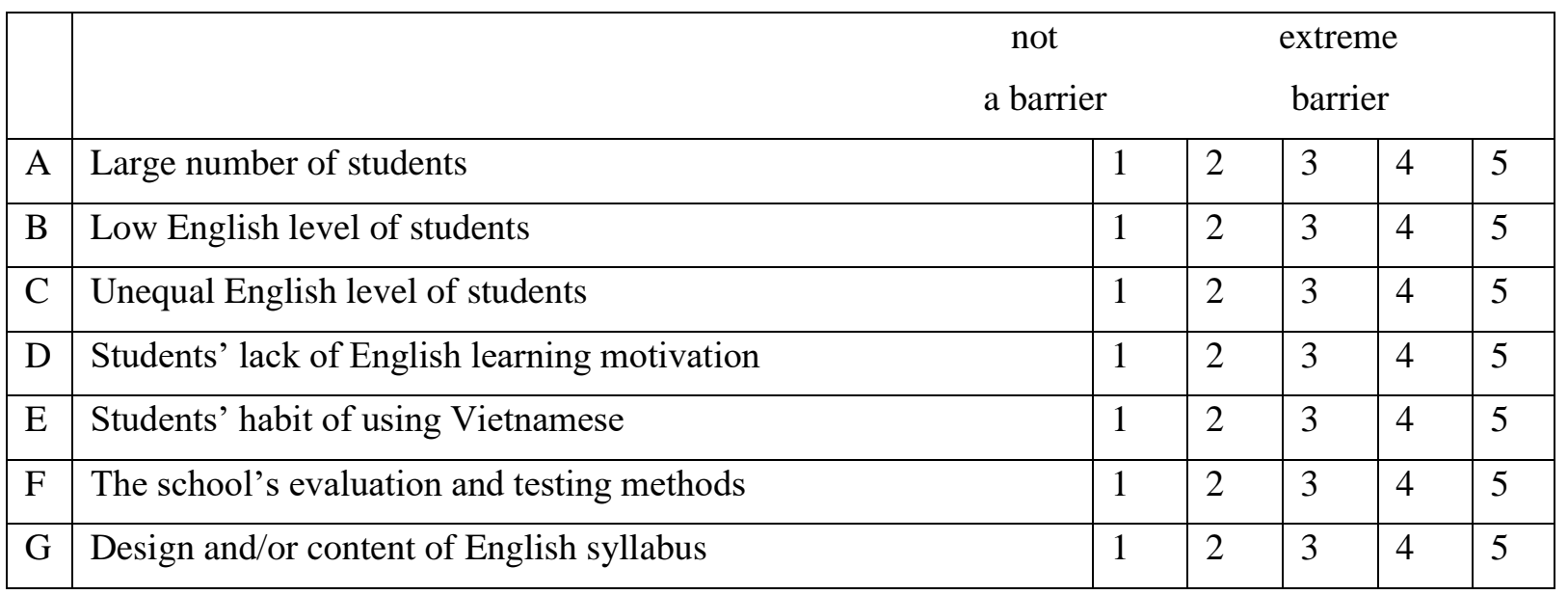




\begin{tabular}{|c|c|c|c|c|c|c|}
\hline $\mathrm{H}$ & Design and/or allocation of English curriculum & 1 & 2 & 3 & 4 & 5 \\
\hline I & The design and/or arrangement of classroom seats and desks & 1 & 2 & 3 & 4 & 5 \\
\hline $\mathrm{J}$ & Lack of supportive teaching tools & 1 & 2 & 3 & 4 & 5 \\
\hline K & 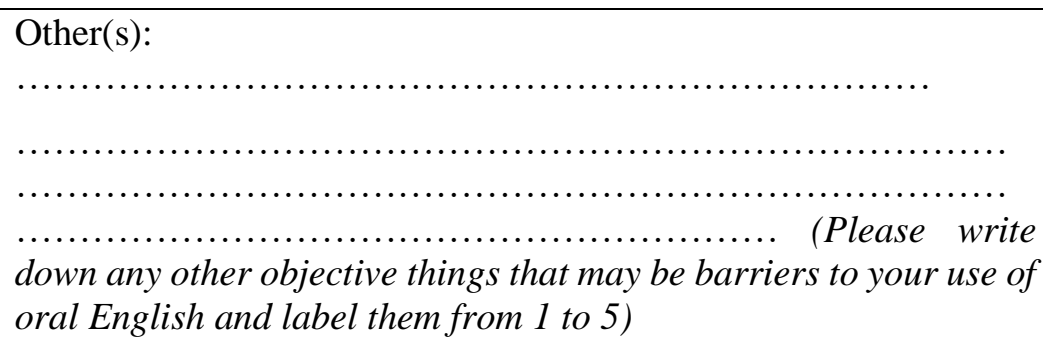 & 1 & 2 & 3 & 4 & 5 \\
\hline
\end{tabular}

13. Following are some subjective aspects that may be barriers to your use of English as the medium of teaching and communication in non-English major classes. Read each aspect and label them from 1 to $\mathbf{5}$, using the rating levels:

\section{1 - Not a barrier at all}

\section{2 - Somewhat of a barrier}

3 - Moderate barrier

4 - Somewhat strong barrier

5 - Extreme barrier

\begin{tabular}{|c|c|c|c|c|c|c|}
\hline & $\begin{array}{c}\text { not } \\
\text { a barric }\end{array}$ & & & & & \\
\hline A & Personal characteristics & 1 & 2 & 3 & 4 & 5 \\
\hline $\mathrm{B}$ & Lack of teaching motivation & 1 & 2 & 3 & 4 & 5 \\
\hline $\mathrm{C}$ & Lack of confidence in speaking English in front of the whole class & 1 & 2 & 3 & 4 & 5 \\
\hline $\mathrm{D}$ & Lack of strong desire to change teaching practices & 1 & 2 & 3 & 4 & 5 \\
\hline $\mathrm{E}$ & Lack of understanding students' perspectives & 1 & 2 & 3 & 4 & 5 \\
\hline $\mathrm{F}$ & Lack of knowledge of teaching strategies & 1 & 2 & 3 & 4 & 5 \\
\hline G & $\begin{array}{l}\text { Lack of practical techniques to apply oral English communication } \\
\text { strategies effectively }\end{array}$ & 1 & 2 & 3 & 4 & 5 \\
\hline $\mathrm{H}$ & $\begin{array}{l}\text { Other(s): } \\
\text { (Please write down any other subjective things that may be } \\
\text { barriers to your } u s e \text { of spoken English and label them from } 1 \text { to } \\
\text { () }\end{array}$ & 1 & 2 & 3 & 4 & 5 \\
\hline
\end{tabular}




\section{Appendix H: Questionnaire for students}

\section{QUESTIONNAIRE FOR STUDENTS}

Directions: This questionnaire is intended to gather your views about what encourages and discourages your understanding of your lecturers' English speaking in classrooms. Please answer each question and return it to Ms. Lien as soon as possible. In the end of this questionnaire, there is a section for you to register for the focus group interview. Please read the instructions carefully and decide if you would like to participate in the next part of the study.

Notes: The lecturers mentioned here mean all those who have been teaching you English at the college.

Please choose the information (tick) that is correct to you.

Gender: $\quad \square$ Male $\square$ Female

Major: $\square$ Electricity, Electronics, Telecommunications

$\square$ E-Commerce, Marketing
$\square$ Computer Network, Information Technology
$\square$ Arts, Graphics Design, Architecture
$\square$ Hotel, Tourism, Catering Service
$\square$ Journalism and Communication
$\square$ Accounting, Audit

Years of learning English $\quad \square$ 1-5 $\quad \square$ 6-10 $\quad \square$ Over 10

\section{Section 1: General information}

1. In general, how would you self-evaluate your English communication skill? (Tick one only)
$\square$ Poor $\square$ Fair
$\square$ Good $\square$ Very good
$\square$ Excellent

2. In general, to what extent are you satisfied with your lecturers' use of spoken English in classroom? (Tick one only)
$\square$ Not at all
$\square$ Slightly
$\square$ Moderately
$\square$ Very
$\square$ Extremely

3. In general, to what extent do you find it difficult to comprehend your lecturer's English speaking in class? (Tick one only)

$\square$ Not at all $\quad \square$ Slightly $\quad \square$ Moderately $\quad \square$ Very $\quad \square$ Extremely

4. Overall, to what extent do you think your lecturers' spoken English in class helps you to improve your communication skills? (Tick one only)

$\square$ Not at all $\square$ Slightly $\square$ Moderately $\square$ Very $\quad \square$ Extremely

4 '. If you chose "Not at all" or "Slightly", what are the possible reasons? (Tick all that apply)

$\square$ a. It goes beyond my level of understanding

$\square$ b. It feels like my lecturers do not care much about whether I understand or not

$\square$ c. It feels like my lecturers do not have appropriate strategies to help us understand

$\square$ d. I myself do not concentrate on my lecturers' speaking English

$\square$ e. Other(s):

4". If you chose "Very" or "Extremely", what are the possible reasons? (Tick all that apply) 
$\square$ a. It matches my level of understanding

$\square$ b. It feels like my lecturers really care about whether I understand or not

$\square$ c. It feels like my lecturers have appropriate strategies to help me understand

$\square$ d. I myself have motivation in listening to and understanding English

$\square$ e. Other(s):

5. Overall, how much do you understand your lecturers' English speaking? (Tick one only)
$\square$ Not at all
$\square$ Slightly
$\square$ Moderately
$\square$ Very
$\square$ Extremely

6. Do you feel motivated, demotivated, or neutral when listening to your lecturers speaking English in class? (Tick either $A, B$, or $C$ )

\section{A. $\square$ Motivated}

Then, what makes you motivated? (Tick all that apply)

$\square$ a. Their speaking style (pronunciation, intonation, stress ...)

$\square$ b. Their ways to make sense his/her speaking (gestures, demonstrations ...)

$\square$ c. Their content of speaking (interesting, new information ...)

$\square$ d. Their use of different supplementary tools (board, sound, videos ...)

$\square$ e. Other(s):

\section{B. $\square$ Demotivated}

Then, what makes you demotivated when listening to your lecturers speaking English? (Tick all that apply)

$\square$ a. Their speaking style (pronunciation, intonation, stress ...)

$\square$ b. Their lack of eye contact and/or interaction

$\square$ c. Their content of speaking (boring, old information ...)

$\square$ d. Their using unfamiliar words and/or structures

$\square$ e. Other(s):

\section{C. $\square$ Neutral}

Then, why do you feel neutral? (Tick all that apply)

$\square$ a. Because their speaking style (pronunciation, intonation, stress ...) is neither interesting nor boring

$\square$ b. Because their content of speaking is neither new nor old

$\square$ c. Because their use of vocabulary is neither easy nor challenging

$\square$ d. Other(s):

\section{Section 2: Your lecturers' English speaking and your understanding}

7. How do you often feel when your lecturers speak English to you in front of the whole class? Can you explain briefly? (Tick all that apply)

$\square$ a. I feel worried because 
$\square$ b. I feel scared because

$\square$ c. I feel excited because

$\square$ d. I feel motivated because

$\square$ e. Other(s): I feel because

8. What helps you best when you do not understand your lecturers' English speaking? (Tick one only)

$\square$ a. They will translate it into Vietnamese and no need for explanation

$\square$ b. They will translate it into Vietnamese with explanation of new words/structures

$\square$ c. They will combine demonstrations and translation into Vietnamese

$\square$ d. They will use different ways with no translation into Vietnamese

$\square$ e. Other idea(s):

9. What teaching tools do you think are the most helpful to your understanding of lecturers' English speaking? (Tick all that apply)

$\square$ a. Paper handouts/cards

$\square$ b. The class board

$\square$ c. The computer

$\square$ d. Real life objects

口 e. Other(s):

10. What strategies do you think are the most helpful to your understanding of the lecturers' English speaking? (Tick all that apply)

$\square$ a. Their using non-verbal communication (e.g. miming, gestures, sound ...)

$\square$ b. Their explaining by speech

$\square$ c. Their writing in words

$\square$ d. Their drawing pictures

$\square$ e. Other(s):

11. Following are some aspects of your lecturers' English speaking in classroom that may be barriers to your comprehension. Read each aspect carefully and label them from 1 to 5, using the rating levels:

1 - Not a barrier at all

2 - Somewhat of a barrier

3 - Moderate barrier

4 - Somewhat strong barrier

5 - Extreme barrier 


\begin{tabular}{|c|c|c|c|c|c|c|}
\hline & & bar & & & & \\
\hline A & Lecturers' speed of speech & 1 & 2 & 3 & 4 & 5 \\
\hline B & Lecturers' volume of voice & 1 & 2 & 3 & 4 & 5 \\
\hline $\mathrm{C}$ & Lecturers' clarity of speech & 1 & 2 & 3 & 4 & 5 \\
\hline $\mathrm{D}$ & Length of lecturers' utterances & 1 & 2 & 3 & 4 & 5 \\
\hline $\mathrm{E}$ & Amount and/or difficulty of vocabulary in lecturers' speech & 1 & 2 & 3 & 4 & 5 \\
\hline $\mathrm{F}$ & Complexity of structures in lecturers' speech & 1 & 2 & 3 & 4 & 5 \\
\hline $\mathrm{G}$ & Lecturers' not translating from English to Vietnamese & 1 & 2 & 3 & 4 & 5 \\
\hline $\mathrm{H}$ & Lecturers' standing too far away from me when speaking & 1 & 2 & 3 & 4 & 5 \\
\hline I & Lack of interaction between you and lecturers & 1 & 2 & 3 & 4 & 5 \\
\hline $\mathbf{J}$ & 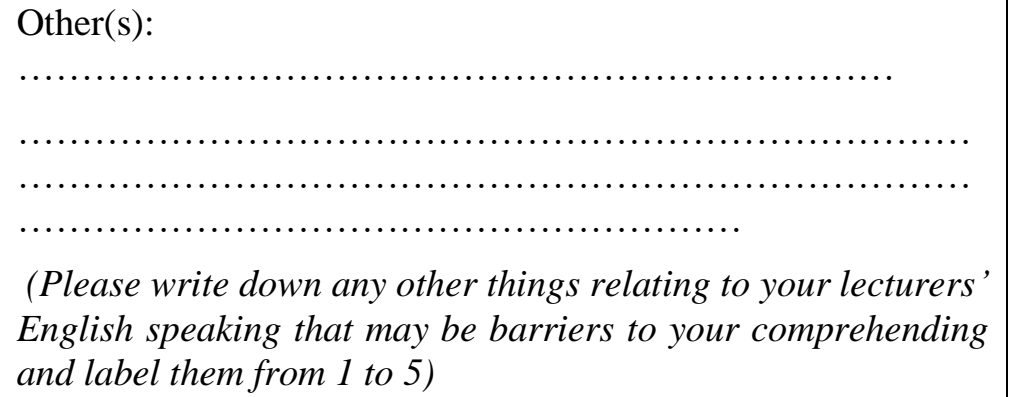 & 1 & 2 & 3 & 4 & 5 \\
\hline
\end{tabular}

12. Following are some subjective factors that may be barriers to your comprehending your lecturers' English speaking in class. Read each aspect carefully and label them from 1 to 5, using the rating levels:

\section{1 - Not a barrier at all}

\section{2 - Somewhat of a barrier}

\section{3 - Moderate barrier}

\section{4 - Somewhat strong barrier}

\section{5 - Extreme barrier}

\begin{tabular}{|c|c|c|c|c|c|c|}
\hline & \multicolumn{2}{|c|}{$\begin{array}{c}\text { not } \\
\text { a barrier }\end{array}$} & \multicolumn{4}{|c|}{$\begin{array}{l}\text { extreme } \\
\text { barrier }\end{array}$} \\
\hline A & My lack of motivation in learning English & 1 & 2 & 3 & 4 & 5 \\
\hline B & My lack of English vocabulary & 1 & 2 & 3 & 4 & 5 \\
\hline $\mathrm{C}$ & My lack of English structures & 1 & 2 & 3 & 4 & 5 \\
\hline $\mathrm{D}$ & My lack of practicing listening to English & 1 & 2 & 3 & 4 & 5 \\
\hline$E$ & My lack of self confidence in listening to English & 1 & 2 & 3 & 4 & 5 \\
\hline $\mathrm{F}$ & My lack of strategies in listening to English & 1 & 2 & 3 & 4 & 5 \\
\hline $\mathrm{G}$ & Other(s): & 1 & 2 & 3 & 4 & 5 \\
\hline
\end{tabular}


(Please write down any other subjective things that may be barriers to your comprehending and label them from 1 to 5)

\section{Section 3: Recruitment of focus group interview members}

Directions: This section is a simple survey to help me choose people to participate in the group interview section (01 interview). If you are interested in participating in this focus group interview (in Vietnamese), please answer the questions. Otherwise, you can skip this section.

13. Are you interested in talking about your experiences of learning English in the college?

$$
\text { Yes } \square \quad N o \square
$$

14. Are you able to articulate your understandings in Vietnamese? $\quad$ Yes $\square \quad$ No $\square$

15. Are you confident in contributing your views in groups and with other students (about 5)?

$$
\text { Yes } \square \quad N o \square
$$

If your answer is "Yes" to all of the $\mathbf{3}$ questions above, then you are the kind of student I need to participate in a focus group interview. If you agree to be a member of this interview, please fill your contact details below.

Name:

Email address:

Phone number:

If any of your answer is "No", then you are not suitable for the focus group interview of this research. However, I highly appreciate your cooperation and thank for your answering this section.

Notes: It is important to remember that there might be more interested and eligible students than needed. So, do not be disappointed if you are not chosen and I highly appreciate your agreement. Whether you will be chosen or not will not affect your scores/ results at school.

\section{Thank you for your cooperation!}




\section{Appendix I: Individual interview protocol for lecturers}

TE WHARE WĀNANGA O TE ŪPOKO O TE IKA A MĀUI

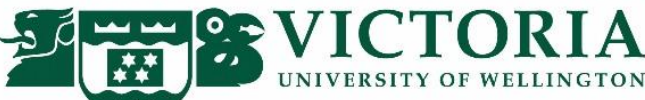

\section{VIETNAMESE TERTIARY TEACHERS' ENGLISH COMMUNICATION IN NON-ENGLISH MAJOR CLASSES - A CASE STUDY}

Semi-structured individual interview protocol for lecturers

\section{Warm-up and Lead-in}

[Thank lecturer ( L) for coming; Introduce myself; Ask if L has any questions; Get L to look through the consent form and sign it; Lead L to Part 1]

Part 1: General information

1. Can you give a very brief introduction about yourself? [Prompt for name/ years of experience in teaching non-English major students/ research expertise ...]

2. Do you think that teaching English with non-major students has special characteristics? What sorts of activities do you find work best in those classes? How do you decide what to teach and how to teach it?

3. What language do you use more, Vietnamese or English in your English classes of non-major? How do you decide which language to use and when? How do students respond when you use English in class? Have you got any memorable experiences while using oral English to teach and communicate with non-English major students?

3. What strategies do you use with non-major students to help them understand when you use oral English? Which are the most effective? Could you share any stories regarding successes and difficulties you have met in applying those strategies to speak English to those students? How have you managed to overcome these challenges?

4. What do you think are the benefits and limitations of using English as the medium for teaching and communication in non-English major classes?

5. Are there things that make it difficult for you to use English as much as you want to? What are they? (policy, classroom layout, own confidence, students' comprehension...)

Part 2: Your recommendations

6. Tell me about any professional learning or training that you have done recently. [Prompt for the content of the training; areas of benefits] Why did you do this training? How has your teaching changed since it? Are there any kinds of professional learning or training courses would you like to complete? [Prompt for dimensions of professional training opportunities, such as contents; types] 
7. In order to make your use of English as a medium of teaching and classroom-based communication in non-English major classes easier and more successful for you, are there any recommendations you would like to make for your school, with regard to the following aspects:

- The school policy: Does your school have particular orientation/ policies to encourage you to use English as a medium of teaching and communication in non-English major classes? What if the "English only policy" was set in those classes? How will you adapt this policy if it is applied?

- The assessment methods: How do the evaluation tests work in your school/ class? Have they encouraged or discouraged your use of English in teaching? Are there any recommendations would you like to make regarding evaluation methods?

- The English syllabus: Are you satisfied with the current English syllabus? Does it facilitate your using oral English in classes? [Probe for dimensions such as Content; Amount of the total course; Allocation of periods...; for explanation and further thoughts] Are there any changes would you like to make to allow more oral English in class?

- The design/ arrangement of classroom seats and desks: What kind of a classroom design/arrangement that makes you feel comfortable to teach in? Have you found the current design/ arrangement facilitative for your oral English communication with students in non-English major classes? If possible, how would you like to redesign it?

- The allocation of students' English levels: Have you ever been in any difficult situations when teaching and communicating with students in English because of the allocation? Have you got any recommendations for this? [Probe for explanation and further thoughts]

- Training students in English listening strategies: Do you think that students should be trained to better listen to their lecturers' English? If yes, how would you recommend them to be trained? [Probe for who will do the training; when; how often ....

- Student surveys: Could you tell me about your latest student survey? [Probe for when; what; how he/she surveyed; how it has helped him/her in practical teaching] Do you often conduct term-beginning regular student surveys as part of teaching plans? What do you often survey? Do you think that there should be such regular student surveys? [Probe for explanation and descriptions such as when; how; what to survey and who to conduct surveys ...]

8. What advice would you give other lecturers teaching English as a non-major subject? [Prompt for level/ amount of oral English, student survey, class layout ...]

9. What advice would you give students of non-English major? [Prompt for skills to improve, learning methods ...]

10. Are there any points would you like to add or clarify? 


\section{Appendix J: Focused group interview protocol for students}

TE WHARE WĀNANGA O TE ŪPOKO O TE IKA A MĀU

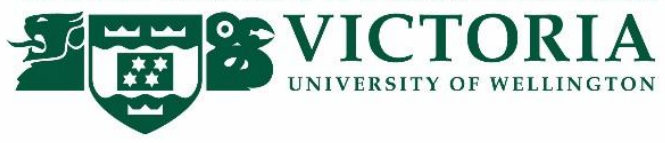

\section{VIETNAMESE TERTIARY TEACHERS' ENGLISH COMMUNICATION IN NON-ENGLISH MAJOR CLASSES - A CASE STUDY}

Focused group semi-structured interview protocol for students

\section{Warm-up and Lead-in}

[Thank students (Ss) for coming; Introduce myself; Ask if Ss have any questions; Get Ss to look through the consent forms and sign them; Briefly restate the principles of the focus group interview; Lead Ss to the interview]

1. Can you give a very brief introduction about yourselves? [Prompt for name/study major/hobby ...]

2. Tell me briefly about your English classes at college. What class activities do you enjoy the most? What aspects of your English class interest you?

3. What language does your lecturer use more in your English classes, English or Vietnamese? Do you like it better when he/she uses English or Vietnamese? Why?

4. How easy is it for you to listen to and understand your lecturers' oral English in class? What makes it easier for you? What makes it more difficult?

5. What motivates you to listen and try to understand when your lecturers speak in English?

6. What skills and strategies do you know about to help you to listen to and understand English? Where did you learn these skills and strategies? Would you like to learn more strategies to help you understand spoken English? What might help you learn these?

7. Do your lecturers ever ask you about how you would like to learn English? How do your lecturers find out about your ideas for how you'd like to learn English? Do you think that there should be regular student surveys about how you would like your English lessons to be? Why or why not?

8. If you could give advice to English lecturers about the things that help you better comprehend their oral English and improve your communication skills, what would you tell them?

- Class layout: What kind of a classroom design/ arrangement that makes you feel comfortable to study in? Have you found the current design/ arrangement facilitative for the classroom communication? If possible, how would you like to redesign it? 
- English only policy: What if the "English only policy" was set in your classes? Would you support this policy? Why/ why not?

- Strategies to improve your comprehension: Are there any strategies that you think effective?

- Other recommendations:

9. If you could make recommendations to your school about some of the things that help you better comprehend your lecturers' oral English and improve your communication skills, what would you recommend?

- Resources/facilities

- English syllabus

- Assessment methods

- Other recommendations

10. What advice would you give other students learning English as a non-major subject?

11. Are there any points would you like to add or clarify? 


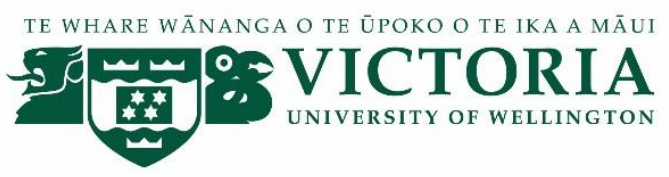

\section{VIETNAMESE TERTIARY TEACHERS' ENGLISH COMMUNICATION \\ IN NON-ENGLISH MAJOR CLASSES - A CASE STUDY \\ Protocol for Focus Group Interview}

Thank you for joining our group discussion. Before we start, there are some points that you should know to make sure that we will all enjoy the interview.

- There are no right or wrong answers; every person's experiences and opinions are important because they can help finding useful solutions and innovations for yourself and future education of English in our country.

- I would like everyone to feel comfortable when sharing information, because the information shared in this group interview is confidential. You should not discuss the opinions and comments made by other participants with anybody outside this room.

- I would like to hear a wide range of ideas, so please talk about whether you agree or disagree with the views shared.

- Everyone should listen respectfully as others are sharing their opinions, but you do not need to agree with others

- The group interview is audio recorded, so please speak one at a time. In order to make sure everyone has opportunity to speak, you will speak one after the other in a circular fashion for the first part of each question and for the other parts, please sign that you would like to speak. You can refuse to speak at any of your turns.

- Please turn off your phones or use silent mode during the discussion.

Please note: Removing an individual's contribution to the group interview is only possible up until the start of the group discussion. Participants can choose to leave the group interview at any time but it will be impossible to withdraw what has been said by them up to the point of them leaving as it will be incorporated into of the general discussion with other participants. 


\section{Appendix K: Principles of questionnaire construction}

Principles of questionnaire construction (Johnson, 2014, p. 193)

1. Make sure the questionnaire items match your research objectives

2. Understand your research participants

3. Use natural and familiar language

4. Write items that are clear, precise, and relatively short

5. Do not use "leading" or "loaded" questions

6. Avoid double-barreled questions

7. Avoid double negatives

8. Determine whether an open-ended or a closed-ended question is needed

9. Use mutually exclusive and exhaustive response categories for closed-ended

10. Consider the different types of response categories available for closed-ended questionnaire items

11. Use multiple items to measure abstract constructs

12. Consider using multiple methods when measuring abstract constructs

13. Use caution if you reverse the wording in some of the items to prevent response sets in multiitem scales

14. Develop a questionnaire that is properly organised and easy for the participant to use

15. Always pilot test questionnaire 


\section{Appendix L: Validity strategies for quantitative data}

Validity strategies for quantitative data in this research (Adapted from Delport and Roestenburg (2011) and Neuman (2003))

\begin{tabular}{lll}
\hline \multicolumn{1}{c}{ Strategies } & \multicolumn{1}{c}{ Benefits } & \multicolumn{1}{c}{ Justifications } \\
\hline $\begin{array}{l}\text { Clear } \\
\text { conceptualisation } \\
\text { of constructs }\end{array}$ & $\begin{array}{l}\text {-To minimise ambiguity } \\
\text {-To eliminate confusion }\end{array}$ & $\begin{array}{l}\text { The researcher will define the key constructs; } \\
\text { and give clear instructions }\end{array}$ \\
$\begin{array}{l}\text { Use of a precise } \\
\text { level of } \\
\text { measurement }\end{array}$ & $\begin{array}{l}\text {-To make sure a close } \\
\text { match between question } \\
\text { contents and research } \\
\text { objectives }\end{array}$ & $\begin{array}{l}\text { The researcher will adopt 15 principles } \\
\text { recommended by Johnson (2014) when } \\
\text { constructing questions }\end{array}$ \\
Use of pilot test & $\begin{array}{l}\text {-To find any mistakes or } \\
\text { gaps in the questions }\end{array}$ & $\begin{array}{l}\text { The researcher will conduct several a pilot tests } \\
\text { before the main study }\end{array}$ \\
\hline
\end{tabular}




\section{Appendix M: Reflections to develop an observation protocol}

Reflections to develop an observation protocol (Adapted from Hay and Singh (2012, p. 224))

How could observations be used in your study?

What would be observed? How would you know you observed it?

How do you envision your role in an observation?

In your observations what degree of involvement do you believe would be appropriate for data collection?

How might your presence influence participants? The setting? 
Appendix N: Dörnyei and Scott's taxonomy

Dörnyei and Scott's communication strategy taxonomy (1997, p.188-194)

\begin{tabular}{|c|c|c|}
\hline STRATEGY & DESCRIPTION & EXAMPLE \\
\hline $\begin{array}{l}\text { 1.Message } \\
\text { abandonment }\end{array}$ & $\begin{array}{l}\text { Leaving a message unfinished } \\
\text { because of some language } \\
\text { difficulty }\end{array}$ & $\begin{array}{l}\text { It is a person er... who is responsible for } \\
\text { a a house, for the block of house... I } \\
\text { don't know... [laughter] }\end{array}$ \\
\hline $\begin{array}{l}\text { 2.Message } \\
\text { reduction } \\
\text { (topic } \\
\text { avoidance) }\end{array}$ & $\begin{array}{l}\text { Reducing the message by } \\
\text { avoiding certain language } \\
\text { structures or topics considered } \\
\text { problematic languagewise } \\
\text { or by leaving out some intended } \\
\text { elements for a lack of } \\
\text { linguistic resources }\end{array}$ & $\begin{array}{l}\text { [Retrospective comment by the } \\
\text { speaker:] I was looking for "satisfied } \\
\text { with a good job, pleasantly tired," and } \\
\text { so on, but } \\
\text { instead I accepted less }\end{array}$ \\
\hline $\begin{array}{l}\text { 3.Message } \\
\text { replacement }\end{array}$ & $\begin{array}{l}\text { Substituting the original message } \\
\text { with a new one because } \\
\text { of not feeling capable of } \\
\text { executing it }\end{array}$ & $\begin{array}{l}\text { [Retrospective comment after saying } \\
\text { that the pipe was broken in the middle } \\
\text { instead of "the screw thread was } \\
\text { broken":] I didn't know "screw thread" } \\
\text { and well, I had to } \\
\text { say something }\end{array}$ \\
\hline $\begin{array}{l}\text { 4.Circumlocution } \\
\text { (paraphrase) }\end{array}$ & $\begin{array}{l}\text { Exemplifying, illustrating or } \\
\text { describing the properties of } \\
\text { the target object or action }\end{array}$ & it becomes water instead of "melt" \\
\hline 5.Approximation & $\begin{array}{l}\text { Using a single alternative lexical } \\
\text { item, such as a superordinate or a } \\
\text { related term, which shares } \\
\text { semantic } \\
\text { features with the target word or } \\
\text { structure }\end{array}$ & plate instead of "bowl" \\
\hline $\begin{array}{l}\text { 6.Use of all- } \\
\text { purpose } \\
\text { words }\end{array}$ & $\begin{array}{l}\text { Extending a general, "empty" } \\
\text { lexical item to contexts where } \\
\text { specific words are lacking }\end{array}$ & $\begin{array}{l}\text { The overuse of thing, stuff, make, do, as } \\
\text { well as words like thingie, what-do-you- } \\
\text { call-it; e.g.: I can't can't work until you } \\
\text { repair my ... thing }\end{array}$ \\
\hline 7.Wordcoinage & $\begin{array}{l}\text { Creating a non-existing L2 word } \\
\text { by applying a supposed L2 rule to } \\
\text { an existing L2 word }\end{array}$ & $\begin{array}{l}\text { [Retrospective comment after using } \\
\text { dejunktion and unjunktion for "street } \\
\text { clearing": I think I approached it in a } \\
\text { very scientific way: from 'junk' I formed } \\
\text { a noun and I tried to add the negative } \\
\text { prefix "de-"; to "unjunk" is to } \\
\text { 'clear the junk' and "unjunktion" is } \\
\text { 'street clearing' }\end{array}$ \\
\hline 8. Restructuring & $\begin{array}{l}\text { Abandoning the execution of a } \\
\text { verbal plan because of language } \\
\text { difficulties, leaving the utterance } \\
\text { unfinished, and communicating } \\
\text { the intended message according } \\
\text { to an } \\
\text { alternative plan }\end{array}$ & $\begin{array}{l}\text { On Mickey's face we can see the... so } \\
\text { he's he's he's wondering. }\end{array}$ \\
\hline $\begin{array}{l}\text { 9.Literal } \\
\text { translation } \\
\text { (transfer) }\end{array}$ & $\begin{array}{l}\text { Translating literally a lexical } \\
\text { item, an idiom, a compound word } \\
\text { or structure from L1/L3 to L2 }\end{array}$ & $\begin{array}{l}\text { I'd made a big fault [translated from } \\
\text { French] }\end{array}$ \\
\hline
\end{tabular}




\begin{tabular}{|c|c|c|}
\hline 10.Foreignising & $\begin{array}{l}\text { Using a L1/L3 word by adjusting } \\
\text { it to L2 phonology (i.e., with a L2 } \\
\text { pronunciation) and/or } \\
\text { morphology }\end{array}$ & $\begin{array}{l}\text { reparate for "repair" [adjusting the } \\
\text { German word } \\
\text { 'reparieren'] }\end{array}$ \\
\hline $\begin{array}{l}\text { 11.Code } \\
\text { switching } \\
\text { (language } \\
\text { switch) }\end{array}$ & $\begin{array}{l}\text { Including L1/L3 words with } \\
\text { L1/L3 pronunciation in L2 } \\
\text { speech; this may involve } \\
\text { stretches of discourse ranging } \\
\text { from single words to whole } \\
\text { chunks and even complete } \\
\text { turns }\end{array}$ & Using the Latin ferrum for "iron" \\
\hline $\begin{array}{l}\text { 12.Use of } \\
\text { Similar sounding } \\
\text { words }\end{array}$ & $\begin{array}{l}\text { Compensating for a lexical item } \\
\text { whose form the speaker } \\
\text { is unsure of with a word (either } \\
\text { existing or non-existing) } \\
\text { which sounds more or less like } \\
\text { the target item }\end{array}$ & $\begin{array}{l}\text { [Retrospective comment explaining why } \\
\text { the speaker used cap instead of "pan":] } \\
\text { Because it was similar to the word } \\
\text { which I wanted to say: "pan"" }\end{array}$ \\
\hline 13.Mumbling & $\begin{array}{l}\text { Swallowing or muttering } \\
\text { inaudibly a word (or part of a } \\
\text { word) whose correct form the } \\
\text { speaker is uncertain about }\end{array}$ & $\begin{array}{l}\text { And uh well Mickey Mouse looks } \\
\text { surprise or sort of XXX [the 'sort of' } \\
\text { marker indicates that the unintelligible } \\
\text { part is not just a mere recording failure } \\
\text { but a strategy] }\end{array}$ \\
\hline 14.Omission & $\begin{array}{l}\text { Leaving a gap when not knowing } \\
\text { a word and carrying on as if it } \\
\text { had been said }\end{array}$ & $\begin{array}{l}\text { then... er... the sun is is... hm sun is... } \\
\text { and the Mickey Mouse.... [Retrospective } \\
\text { comment: I didn't know what 'shine' } \\
\text { was.] }\end{array}$ \\
\hline 15.Retrieval & $\begin{array}{l}\text { In an attempt to retrieve a lexical } \\
\text { item saying a series of incomplete } \\
\text { or wrong forms or structures } \\
\text { before reaching the optimal form }\end{array}$ & It's brake er... it's broken broked broke \\
\hline 16a.Self-repair & $\begin{array}{l}\text { Making self-initiated corrections } \\
\text { in one's own speech }\end{array}$ & $\begin{array}{l}\text { then the sun shines and the weather get } \\
\text { be... gets better }\end{array}$ \\
\hline 16b.Other repair & $\begin{array}{l}\text { Correcting something in the } \\
\text { interlocutor's speech }\end{array}$ & $\begin{array}{l}\text { Speaker:... because our tip went } \\
\text { wrong... [...] Interlocutor: Oh, you } \\
\text { mean the tap. S: Tap, tap... }\end{array}$ \\
\hline 17.Selfrephrasing & $\begin{array}{l}\text { Repeating a term, but not quite as } \\
\text { it is, but by adding something or } \\
\text { using paraphrase }\end{array}$ & $\begin{array}{l}\text { I don't know the material... what it's } \\
\text { made of... }\end{array}$ \\
\hline $\begin{array}{l}\text { 18.Over- } \\
\text { explicitness } \\
\text { (waffling) }\end{array}$ & $\begin{array}{l}\text { Using more words to achieve a } \\
\text { particular communicative goal } \\
\text { than what is considered normal in } \\
\text { similar L1 situations }\end{array}$ & $\begin{array}{l}\text { (This CS was not included in Dörnyei \& } \\
\text { Scott's, 1995, taxonomy) }\end{array}$ \\
\hline $\begin{array}{l}\text { 19.Mime } \\
\text { (nonlinguistic/ } \\
\text { paralinguistic } \\
\text { strategies) }\end{array}$ & $\begin{array}{l}\text { Describing whole concepts } \\
\text { nonverbally, or accompanying } \\
\text { a verbal strategy with a visual } \\
\text { illustration }\end{array}$ & $\begin{array}{l}\text { [Retrospective comment:] I was miming } \\
\text { here, to put it out in front of the house, } \\
\text { because I couldn't remember the word }\end{array}$ \\
\hline 20.Use of fillers & $\begin{array}{l}\text { Using gambits to fill pauses, to } \\
\text { stall, and to gain time in order to } \\
\text { keep the communication channel } \\
\text { open and } \\
\text { maintain discourse at times of } \\
\text { difficulty }\end{array}$ & $\begin{array}{l}\text { Examples range from very short } \\
\text { structures such as well; you know; } \\
\text { actually; okay, to longer phrases such as } \\
\text { this is rather difficult to explain; well, } \\
\text { actually, it's a good question }\end{array}$ \\
\hline
\end{tabular}




\begin{tabular}{|c|c|c|}
\hline 21a.Self repetition & $\begin{array}{l}\text { Repeating a word or a string of } \\
\text { words immediately after they } \\
\text { were said }\end{array}$ & $\begin{array}{l}\text { [Retrospective comment:] I wanted to } \\
\text { say that it was made of concrete but I } \\
\text { didn't know 'concrete' and this is why } \\
\text { "which was made, which was made" } \\
\text { was said twice }\end{array}$ \\
\hline $\begin{array}{l}\text { 21b.Other } \\
\text { repetition }\end{array}$ & $\begin{array}{l}\text { Repeating something the } \\
\text { interlocutor said to gain time }\end{array}$ & $\begin{array}{l}\text { Interlocutor: And could you tell me the } \\
\text { diameter of the pipe? The diameter. } \\
\text { Speaker: The diameter? It's about er... } \\
\text { maybe er... five centimeters. }\end{array}$ \\
\hline $\begin{array}{l}\text { 22.Feigning } \\
\text { understanding }\end{array}$ & $\begin{array}{l}\text { Making an attempt to carry on the } \\
\text { conversation in spite of not } \\
\text { understanding something by } \\
\text { pretending to understand }\end{array}$ & $\begin{array}{l}\text { Interlocutor: Do you have the rubber } \\
\text { washer? Speaker: The rubber washer? } \\
\text {... No I don't. [Retrospective comment: } \\
\text { I didn't know the meaning of the word, } \\
\text { and finally I managed to say I had no } \\
\text { such thing.] }\end{array}$ \\
\hline $\begin{array}{l}\text { 23.Verbal } \\
\text { strategy } \\
\text { markers }\end{array}$ & $\begin{array}{l}\text { Using verbal marking phrases } \\
\text { before or after a strategy to signal } \\
\text { that the word or structure does } \\
\text { not carry the } \\
\text { intended meaning perfectly in the } \\
\text { L2 code }\end{array}$ & $\begin{array}{l}\text { E.g.: (strategy markers in bold): } \\
\text { (a) marking a } \\
\text { circumlocution: On the next picture... I } \\
\text { don't really know what's it called in } \\
\text { English... it's uh this kind of bird that... } \\
\text { that can be found in a clock that strikes } \\
\text { out or [laughs] comes out when the } \\
\text { clock strikes }\end{array}$ \\
\hline $\begin{array}{l}\text { 24a.Direct } \\
\text { appeal for help }\end{array}$ & $\begin{array}{l}\text { Turning to the interlocutor for } \\
\text { assistance by asking an explicit } \\
\text { question concerning a gap in } \\
\text { one's L2 knowledge }\end{array}$ & $\begin{array}{l}\text { it's a kind of old clock so when it } \\
258 \text { truck ser... I don't know, one, two, } \\
\text { or three 'clock then a bird is coming } \\
\text { out. What's the name? }\end{array}$ \\
\hline $\begin{array}{l}\text { 24b.Indirect } \\
\text { appeal for help }\end{array}$ & $\begin{array}{l}\text { Trying to elicit help from the } \\
\text { interlocutor indirectly by } \\
\text { expressing lack of a needed L2 } \\
\text { item either verbally or } \\
\text { nonverbally }\end{array}$ & $\begin{array}{l}\text { I don't know the name... [rising } \\
\text { intonation, pause, eye contact] }\end{array}$ \\
\hline $\begin{array}{l}\text { 25.Asking for } \\
\text { repetition }\end{array}$ & $\begin{array}{l}\text { Requesting repetition when not } \\
\text { hearing or understanding } \\
\text { something properly }\end{array}$ & Pardon? What? \\
\hline $\begin{array}{l}\text { 26.Asking for } \\
\text { clarification }\end{array}$ & $\begin{array}{l}\text { Requesting explanation of an } \\
\text { unfamiliar meaning structure }\end{array}$ & $\begin{array}{l}\text { What do you mean?, You saw what? } \\
\text { Also 'question repeats,' that is, echoing } \\
\text { a word or a structure with a question } \\
\text { intonation }\end{array}$ \\
\hline $\begin{array}{l}\text { 27.Asking for } \\
\text { confirmation }\end{array}$ & $\begin{array}{l}\text { Requesting confirmation that one } \\
\text { heard or understood something } \\
\text { correctly }\end{array}$ & $\begin{array}{l}\text { Repeating the trigger in a 'question } \\
\text { repeat' or asking a full question, such as } \\
\text { You said...?, You mean...?, Do you } \\
\text { mean...? }\end{array}$ \\
\hline 28.Guessing & $\begin{array}{l}\text { Guessing is similar to a } \\
\text { confirmation request but the latter } \\
\text { implies a greater degree of } \\
\text { certainty regarding the key word, } \\
\text { whereas guessing involves real } \\
\text { indecision }\end{array}$ & $\begin{array}{l}\text { E.g.: Oh. It is then not the washing } \\
\text { machine. Is it a sink? }\end{array}$ \\
\hline $\begin{array}{l}\text { 29.Expressing } \\
\text { non- } \\
\text { understanding }\end{array}$ & $\begin{array}{l}\text { Expressing that one did not } \\
\text { understand something } \\
\text { properly either verbally or } \\
\text { nonverbally }\end{array}$ & $\begin{array}{l}\text { Interlocutor: What is the diameter of the } \\
\text { pipe? Speaker: The diameter? I: The } \\
\text { diameter. S: I don't know this thing. }\end{array}$ \\
\hline
\end{tabular}




\begin{tabular}{|c|c|c|}
\hline & & $\begin{array}{l}\text { I: How wide is the pipe? Also, puzzled } \\
\text { facial expressions, frowns and various } \\
\text { types of mime and gestures. }\end{array}$ \\
\hline $\begin{array}{l}\text { 30.Interpretive } \\
\text { summary }\end{array}$ & $\begin{array}{l}\text { Extended paraphrase of the } \\
\text { interlocutor's message to } \\
\text { check that the speaker has } \\
\text { understood correctly }\end{array}$ & $\begin{array}{l}\text { So the pipe is broken, basically, and you } \\
\text { don't know what } \\
\text { to do with it, right? }\end{array}$ \\
\hline $\begin{array}{l}\text { 31.Comprehension } \\
\text { check }\end{array}$ & $\begin{array}{l}\text { Asking questions to check that } \\
\text { the interlocutor can follow you }\end{array}$ & $\begin{array}{l}\text { And what is the diameter of the pipe? } \\
\text { The diameter. Do } \\
\text { you know what the diameter is? }\end{array}$ \\
\hline $\begin{array}{l}\text { 32.Own-accuracy } \\
\text { check }\end{array}$ & $\begin{array}{l}\text { Checking that what you said was } \\
\text { correct by asking a } \\
\text { concrete question or repeating a } \\
\text { word with a question } \\
\text { intonation }\end{array}$ & $\begin{array}{l}\text { I can see a huge snow... snowman? } \\
\text { Snowman in the garden }\end{array}$ \\
\hline $\begin{array}{l}\text { 33a.Response: } \\
\text { repeat }\end{array}$ & $\begin{array}{l}\text { Repeating the original trigger or } \\
\text { the suggested corrected form } \\
\text { (after an other-repair) }\end{array}$ & See the example of other-repair \\
\hline $\begin{array}{l}\text { 33b.Response: } \\
\text { repair }\end{array}$ & $\begin{array}{l}\text { Providing other-initiated self- } \\
\text { repair }\end{array}$ & $\begin{array}{l}\text { Speaker: The water was not able to get } \\
\text { up and I... } \\
\text { Interlocutor: Get up? Where? S: Get } \\
\text { down }\end{array}$ \\
\hline $\begin{array}{l}\text { 33c.Response: } \\
\text { rephrase }\end{array}$ & Rephrasing the trigger & $\begin{array}{l}\text { Interlocutor: And do you happen to } \\
\text { know if you have the rubber washer? } \\
\text { Speaker: Pardon? I: The rubber } \\
\text { washer... it's the thing which is in the } \\
\text { pipe }\end{array}$ \\
\hline $\begin{array}{l}\text { 33d.Response: } \\
\text { expand }\end{array}$ & $\begin{array}{l}\text { Putting the problem word/issue } \\
\text { into a larger context }\end{array}$ & $\begin{array}{l}\text { Interlocutor: Do you know maybe er } \\
\text { what the diameter of the pipe is? } \\
\text { Speaker: Pardon? I: Diameter, this is er } \\
\text { maybe you learnt mathematics and you } \\
\text { sign er with th this part } \\
\text { of things }\end{array}$ \\
\hline $\begin{array}{l}\text { 33e.Response: } \\
\text { confirm }\end{array}$ & $\begin{array}{l}\text { Confirming what the interlocutor } \\
\text { has said or suggested }\end{array}$ & $\begin{array}{l}\text { Interlocutor: Uh, you mean under the } \\
\text { sink, the pipe? For the... Speaker: Yes. } \\
\text { Yes. }\end{array}$ \\
\hline $\begin{array}{l}\text { 33f.Response: } \\
\text { reject }\end{array}$ & $\begin{array}{l}\text { Rejecting what the interlocutor } \\
\text { has said or suggested without } \\
\text { offering an alternative solution }\end{array}$ & Interlocutor: Is it plastic? Speaker: No. \\
\hline
\end{tabular}




\section{Appendix O: Transcription symbols}

\begin{tabular}{ll}
\hline Symbol & Explanation \\
\hline Ellen/Amy/Polly/Olive/ Una: & Lecturers' pseudonym names \\
L: & Lecturer \\
S: & One student is speaking \\
Ss: & Two or more students are speaking \\
$\uparrow$ & rising shift in intonation \\
$\downarrow$ & falling shift in intonation \\
AA & Turning up the volume \\
aa & Turing down the volume \\
$>$ speech $<$ & Speeding up \\
$<$ speech $>$ & Slowing down \\
$()$. & Short pause $(05-2$ seconds) \\
$(\ldots)$ & Long pause (>=3 seconds) \\
$($ text $)$ & Nonverbal behaviour \\
$\ldots$ & Deleted texts \\
$(\ldots)$. & Unclear speech \\
$/$ & Phonetic transcription \\
{$[$ text $]$} & English translation of Vietnamese L1 \\
$\rightarrow($ text $)$ & Purpose/ function of strategies \\
speech & To draw attention that an analyst wishes to \\
& discuss \\
$($ text $))$ & The researcher's comments \\
\hline
\end{tabular}




\section{Appendix P: Examples of communication strategies' episode}

\section{Example 1:}

L: Ok now ((use of fillers)) look at these. Now class, tell me what tense what tense is it? What tense is it? ${ }^{\uparrow}(($ repetition strategy at sentential level $)) \rightarrow($ draw attention $)$

Ss: (silent)

L: You know tense? $<$ You know tense $>($ (interaction strategy $)) \rightarrow($ draw attention, ask for comprehension)

Ss: (laughing) (...?)

No. I don't ask you about my age, có hiểu không? [Do you understand?] ((codeswitching)) $\rightarrow$ (comprehension check)

\section{Example 2:}

L: Now ((verbal markers)) stop (clap hands) ((nonverbal strategy)) $\rightarrow$ (draw attention). Group 1, group 2 be quick. Let's see how many words you got for each group. Who can help me? (raise her hand) ((nonverbal strategy)) $\rightarrow$ (to demonstrate the meaning of "help")

Ss: (raise their hands)

L: You, my love, dear. Come here please! Lên đây em [come here, dear] ((translation strategy)) $\rightarrow$ (nudging and encouraging)

\section{Example 3:}

L: Yes, what have you tried? tell me please AA what have you tried? ((self-repetition)) $\rightarrow$ (seek for students' responses)

Ss: chicken

L: yes everyone chicken ((other repetition)) Trung

S: ice cream

L: What flavour do you like? If you eat ice cream what ... flavour AA of ice cream ((repetition at word level)) do you like?

Ss: (...?)

L: Pardon? Do you like strawberry, vanilla, chocolate, apple or whatever? ((simplification by related terms $)) \rightarrow$ (clarify meaning) 


\title{
Appendix Q: Examples of analysis of lecturer and student interview excerpts
}

\author{
(Translated to English)
}

\section{Identifying and coding phases}

\begin{tabular}{l}
\hline Data evidence \\
For non-English major students in my school, their entry \\
level is at collegiate level, meaning their English level is \\
really low because students do not have an entry English \\
test but are accepted based on their general high school \\
score report. The second problem/challenge is \\
consistency. Students come from many different regions; \\
in some areas, children begin to learn English at grade 6, \\
while in other regions, children do not study English until \\
they enter grade 10. The learning and teaching conditions \\
in different places vary as well. Since students' levels and \\
English background are heterogeneous within one \\
class/group; lecturers do not know how much and what \\
the most effective way to use English in those classes is. \\
Most of the students still have a passive learning method, \\
sitting in one place, listening, and writing notes. There are \\
so many students in one class. These also make it very \\
difficult for teachers to organize classroom activities. \\
Then, many of them cannot speak English; and some do \\
not want to. When I ask them questions in English, they \\
normally answer in Vietnamese; and only switch to \\
English until I get angry with them. They only speak \\
when I call their names but not actively participate in the \\
lessons. So many teaching hours also makes teachers \\
have no time to invest more in the lessons (Amy)
\end{tabular}

\section{Data evidence}

I study with [student name]; so the actual ratio of my lecturer's English is $40 \%$ and Vietnamese being $60 \%$; but I want her to increase English level to $70 \%$; and reduce Vietnamese to $30 \%$. At first lecturers should speak slowly; really slowly. When students do not understand, they can either explain by using body language or intonation and giving examples. If those ways are not effective, then she can explain in Vietnamese. I think it will be better.

I think the problem here is not whether we can understand English immediately; we can, if they speak slowly and explain the words. We may not get used to English at once; but when it is repeated two times three times, we will surely remember it. If students have some preparation in advance and lecturers' repetition and teaching, we can learn better. Besides, she should not use long or grammatically correct utterances; just speak with short words and focus on content only. Long sentences make students who are ignorant and lazy do not want to listen. (IS4)

\begin{tabular}{|l|}
\hline Coded themes \\
\hline Students' low English level \\
\hline $\begin{array}{l}\text { Students' inhomogeneous English } \\
\text { levels }\end{array}$ \\
\hline $\begin{array}{l}\text { Different length and conditions of } \\
\text { previous English learning }\end{array}$ \\
\hline Ineffective use of English \\
\hline Students' passive learning habits \\
\hline Large class \\
\hline Ineffective classroom management \\
\hline Students' negative learning \\
\hline behaviours
\end{tabular}

Coded themes

Estimating lecturers' L1 and L2 amount

Expecting for more L2 and less L1

Expecting for lower speech

Expecting for nonverbal and simplification

L1 should be the last option

Their low comprehension is not an issue

Believing in the effectiveness of repetition

Expecting for shorter and more focused speech

Negative effects of long and academic speech 
Developing and naming overarching ideas from sub-ideas

\begin{tabular}{|c|c|c|c|}
\hline Sub-themes & Bigger themes & Bigger themes & $\begin{array}{c}\text { Overarching } \\
\text { themes }\end{array}$ \\
\hline Students' low English level & \multirow{2}{*}{$\begin{array}{l}\text { Students' } \\
\text { proficiency levels }\end{array}$} & \multirow{4}{*}{$\begin{array}{l}\text { Student- } \\
\text { related }\end{array}$} & \multirow{8}{*}{$\begin{array}{l}\text { factors } \\
\text { that affect } \\
\text { lecturers' oral } \\
\text { communication }\end{array}$} \\
\hline $\begin{array}{l}\text { Students' inhomogeneous English } \\
\text { levels }\end{array}$ & & & \\
\hline Students' passive learning habits & \multirow{2}{*}{$\begin{array}{l}\text { Students' learning } \\
\text { habits and attitudes }\end{array}$} & & \\
\hline $\begin{array}{l}\text { Students' negative learning } \\
\text { behaviours }\end{array}$ & & & \\
\hline No entry tests & & \multirow{4}{*}{ Contexts } & \\
\hline $\begin{array}{l}\text { Different length and conditions of } \\
\text { previous English learning }\end{array}$ & & & \\
\hline Big class & & & \\
\hline Heavy workload & & & \\
\hline $\begin{array}{l}\text { Estimating lecturers' L1 and L2 } \\
\text { amount }\end{array}$ & \multirow[t]{3}{*}{ L1 and L2 use } & & \multirow{7}{*}{$\begin{array}{l}\text { Students' } \\
\text { perception of } \\
\text { lecturers' use of } \\
\text { oral } \\
\text { communication }\end{array}$} \\
\hline $\begin{array}{l}\text { Expecting for more L2 and less } \\
\text { L1 }\end{array}$ & & & \\
\hline L1 should be the last option & & & \\
\hline Expecting for lower speech & \multirow{2}{*}{$\begin{array}{l}\text { Communication } \\
\text { styles }\end{array}$} & & \\
\hline $\begin{array}{l}\text { Expecting for shorter and more } \\
\text { focused speech }\end{array}$ & & & \\
\hline $\begin{array}{l}\text { Expecting for nonverbal and } \\
\text { simplification }\end{array}$ & \multirow{2}{*}{$\begin{array}{l}\text { Communication } \\
\text { strategies }\end{array}$} & & \\
\hline Believing in the role of repetition & & & \\
\hline
\end{tabular}

